\title{
PORE PRESSURE PARAMETERS AND CYCLIC RESISTANCE UNDER GENERALIZED LOADING
}

\author{
A thesis submitted to \\ the Faculty of Graduate and Postdoctoral Affairs \\ in Partial Fulfillment of the requirements for the degree \\ Master of Applied Science
}

by

Sinthujan Navaratnavel

B. Sc. Eng, University of Peradeniya, Sri Lanka (2010)

Department of Civil and Environmental Engineering Carleton University

Ottawa-Carleton Institute of Civil and Environmental Engineering

August 2013

C2013 Sinthujan Navaratnavel 


\begin{abstract}
A systematic study was undertaken to assess the effects of the magnitude and nature of principal stress rotation on the cyclic resistance of sands. Fraser River sand specimens reconstituted using water pluviation were subjected to cyclic loading at a given CSR but with different magnitudes of stress rotation $\left(\Delta \alpha_{\sigma}\right)$ about different initial inclinations $\left(\alpha_{\sigma c}\right)$. Most of the tests were conducted at plane strain conditions and a limited number under axisymmetric conditions.
\end{abstract}

Test results over a range of $\alpha_{\sigma c}$ and $\Delta \alpha_{\sigma}$ indicate that that the weakest cyclic resistance at a given initial $\alpha_{\sigma c}$ always correspond to $45^{\circ}$ stress rotation, and for a given magnitude of stress rotation (fixed $\Delta \alpha_{\sigma}$ ) the liquefaction resistance generally depends on the magnitude of the torsional shear stress applied on the horizontal plane. These tests indicate that in addition to the orientation of the major principal stress (which is recognized in the literature), the magnitude and time history of the shear stress on the bedding plane (not considered in the literature) plays a critical role in the cyclic strength of the soils.

A limited number of experiments were carried out to investigate the applicability of the Skempton \& Henkel pore pressure formulations for generalized 3D initial stress states. Skempton's pore pressure parameter formulations for isotropic material have been extended to cross anisotropic materials, and it was shown that the principles are applicable even for cross anisotropic materials under generalized initial loading conditions. Further, it is shown that shear induced excess pore water pressure decreases with the magnitude of initial shear stress (i.e., inversely related to $k_{c}$ ) and increase with the inclination of the major principal stress with respect to the deposition direction. 


\section{TABLE OF CONTENTS}

$\begin{array}{ll}\text { ABSTRACT ii } & \text { ii }\end{array}$

TABLE OF CONTENTS $\quad$ iii

LIST OF FIGURES Di vi

LIST OF TABLES $\quad$ xiii

LIST OF SYMBOLS X xiv

ACKNOWLEDGEMENTS Xviii

1. INTRODUCTION 1

1.1 Liquefaction Phenomenon 1

1.2 Evaluating Liquefaction Susceptibility 2

1.3 Motivation for the Research Using HCT Device 3

1.4 Objective of the Research $\quad 6$

$\begin{array}{ll}1.5 & \text { Organization of the Thesis }\end{array}$

2. LITERATURE REVIEW 9

$\begin{array}{ll}2.1 & \text { Introduction }\end{array}$

2.2 Anisotropy in Sands $\quad 9$

2.3 Undrained Monotonic Behaviour of Sand 11

2.4 Undrained Cyclic Behaviour of Sand 15

2.5 Effect of Principal Stress Direction 22

2.6 Pore Pressure Generation in Saturated Soils 26

3. LABORATORY SOIL TESTING DEVICES 32

3.1 Introduction $\quad 32$

3.2 Triaxial Device 33 
3.4 Hollow Cylinder Torsional Shear Device 37

3.4.1 Features of the Carleton University HCT device 37

$\begin{array}{lll}\text { 3.4.2 Stress and strains in a HCT specimen } & 40\end{array}$

3.4.3 Loading system of the HCT device 43

3.4.4 Measuring system of the HCT device 47

3.4.5 Data acquisition system of the CU HCT device 48

4. EXPERIMENTAL WORK $\quad 50$

$\begin{array}{lr}4.1 \text { Introduction } & 50\end{array}$

$\begin{array}{lr}\text { 4.2 Material Tested } & 50\end{array}$

4.3 Specimen Preparation $\quad 52$

4.3.1 Preliminary steps of specimen preparation 53

$\begin{array}{lll}\text { 4.3.2 Specimen preparation steps } & 54\end{array}$

$\begin{array}{ll}\text { 4.4 Void Ratio Calculation } & 60\end{array}$

5. EFFECTS OF INITIAL DIRECTIONS AND LEVEL OF ROTATIONS OF PRINCIPAL STRESS ON LIQUEFACTION RESISTANCE 62

$\begin{array}{ll}5.1 \text { Introduction } & 62\end{array}$

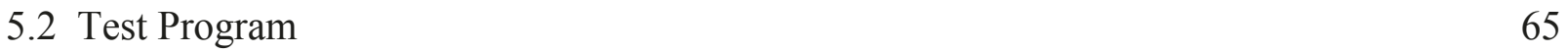

5.3 Monotonic Response under Principal Stress Rotation 66

5.4 Influence of $\Delta \alpha_{\sigma}$ on Cyclic Behavior of Sand 74

5.4.1 Behaviour for $\alpha_{\sigma c}=0^{\circ} \quad 74$

5.4.2 Behaviour for $\alpha_{\sigma c}=30^{\circ} \quad 91$

5.4.3 Behaviour for $\alpha_{\sigma c}=45^{\circ} \quad 95$ 
5.4.4 Behaviour for $\alpha_{\sigma c}=60^{\circ} \quad 98$

5.4.5 Behaviour for $\alpha_{\sigma c}=90^{\circ} \quad 101$

5.5 Influence of $\alpha_{\sigma c}$ on Cyclic Behavior of Sand 104

5.5.1 Behaviour for $\Delta \alpha_{\sigma}=0^{\circ} \quad 104$

5.5.2 Behaviour for $\Delta \alpha_{\sigma}=30^{\circ} \quad 107$

5.5.3 Behaviour for $\Delta \alpha_{\sigma}=45^{\circ} \quad 109$

5.5.4 Behaviour for $\Delta \alpha_{\sigma}=60^{\circ} \quad 111$

5.5.5 Behaviour for $\Delta \alpha_{\sigma}=90^{\circ} \quad 113$

$\begin{array}{ll}\text { 5.6 Summary } & 113\end{array}$

6. PORE PRESSURE GENERATION UNDER GENERALIZED LOADING 117

$\begin{array}{ll}6.1 \text { Introduction } & 117\end{array}$

6.2 Analytical Formulations for Pore Pressure Generation in an Elastic Material 118

6.2.1 Hydrostatic stress increment in a transversely isotropic soil 118

6.2.2 Non-hydrostatic stress increment in a transversely isotropic soil $\quad 120$

$\begin{array}{ll}6.3 \text { Test Program } & 124\end{array}$

6.4 Skempton's B Parameter under Generalized Loading 129

6.5 Shear Induced Pore Pressure Generation: Skempton's $A$ \& Henkel's $a$

$\begin{array}{ll}\text { 6.6 Discussion } & 145\end{array}$

$\begin{array}{ll}\text { 7. SUMMARY AND CONCLUSIONS } & 147\end{array}$

7.1 Principal Stress Rotation \& Liquefaction $\quad 147$

7.2 Pore Pressure Generation \& 3D Stress State $\quad 150$

7.3 Recommendations for Future Research 152

8 REFERENCE 153 


\section{LIST OF FIGURES}

Fig. 1.1 Limiting equilibrium stress state beneath an embankment

Fig. 1.2 Rotation of principal stresses in a soil element of level ground during shear wave propagation (After Logeswaran 2010)

Fig. 2.1 Characteristic response of sand under undrained static loading (After Chern 1985) 12

Fig. 2.2 True liquefaction of sand during cyclic undrained shear (After Vaid and Chern 1985)

Fig. 2.3 Limited liquefaction of sand during cyclic undrained shear

(After Vaid and Chern 1985)

Fig. 2.4 Cyclic mobility in sand during cyclic undrained shear with transient state of zero effective stress (After Vaid and Chern 1985)

Fig. 2.5 Cyclic mobility in sand during cyclic undrained shear without transient state of zero effective stress (After Vaid and Chern 1985)

Fig. 2.6 Increment of principal stresses in axisymmetric soil element during rotation of principal stress (After Law and Holtz 1978)

Fig. 3.1 The stress representation of triaxial in Mohr's circle

Fig. 3.2 Schematic layout of HCT device at Carleton University (After Logeswaran 2010) 39

Fig. 3.3 Porous stones embedded into end platen with radial ribs (After Logeswaran 2010) 40

Fig. 3.4 Surface tractions and stress state of a soil element in a HCT specimen

Fig. 3.5 Pressure and volume control of inner chamber (After Logeswaran 2010) 
Fig. 3.6 Pressure control of external chamber (After Logeswaran 2010)

Fig. 3.7 Vertical stress and vertical displacement control (After Logeswaran 2010) 45

Fig. 3.8 Vertical and Torsional load applying system (After Logeswaran 2010) 46

Fig. 3.9 Back pressure and specimen volume control (After Logeswaran 2010) 47

Fig. 3.10 Torsional displacement measurement in the HCT device 49

Fig. 4.1 Grain size distribution of Fraser river sand used 51

Fig. 4.2 HCT specimen preparation by water pluviation technique (After Sayao 1989) 56

Fig. 4.3 Details of HCT specimen setup (After Logeswaran 2010) 57

$\begin{array}{lll}\text { Fig. 5.1 Cyclic shear stress and direction of principal stresses } & 63\end{array}$

Fig 5.2 Effective stress path, deviator stress and major principal stress direction variation with maximum shear strain

Fig. 5.3 Effective stress path, deviator stress and major principal stress direction $\begin{array}{ll}\text { variation with maximum shear strain } & 71\end{array}$

$\begin{array}{lll}\text { Fig. 5.4 Variation of stress state at PT and MO state } & 72\end{array}$

Fig. 5.5 Variation of phase transformation strength with $\Delta \alpha_{\sigma}$ and $\alpha_{\sigma}$ at failure 73

Fig. 5.6 Shear stress on the horizontal plane during smooth rotation of $\alpha_{\sigma}$ from $\alpha_{\sigma c}=0^{\circ} \quad 76$

Fig. 5.7 Variation of deviatoric stress, excess pore pressure and maximum shear strain with number of cycles for $\Delta \alpha_{\sigma}=0^{\circ}$

Fig. 5.8 Variation of deviatoric stress, excess pore pressure and maximum shear strain with number of cycles for $\Delta \alpha_{\sigma}=60^{\circ}$ 78

Fig. 5.9 Variation of total vertical stress, effective vertical stress and torsional shear stress 
applied on the horizontal plane with number of cycles for $\Delta \alpha_{\sigma}=0^{\circ}$

Fig. 5.10 Variation of total vertical stress, effective vertical stress and torsional shear stress applied on the horizontal plane with number of cycles for $\Delta \alpha_{\sigma}=60^{\circ}$

Fig. 5.11 Variation of vertical strain, radial strain and torsional shear strain applied on the horizontal plane with number of cycles for $\Delta \alpha_{\sigma}=0^{\circ}$

Fig. 5.12 Variation of vertical strain, radial strain and torsional shear strain applied on the horizontal plane with number of cycles for $\Delta \alpha_{\sigma}=60^{\circ}$

Fig. 5.13 Stress path of Fraser River sand during cyclic loading of $\frac{\sigma_{d}}{2 \sigma_{3 c}^{\prime}}=0.20$ where $\alpha_{\sigma}$ was kept along vertical deposition direction

Fig. 5.14 Stress path of Fraser River sand during rotation of $\alpha_{\sigma}$ between $-30^{\circ}$ and $+30^{\circ}$

Fig. 5.15 Stress path of Fraser River sand during rotation of $\alpha_{\sigma}$ between $-45^{\circ}$ and $+45^{\circ} \quad 86$

Fig. 5.16 Stress path of Fraser River sand during rotation of $\alpha_{\sigma}$ between $-60^{\circ}$ and $+60^{\circ} \quad 86$

Fig. 5.17 Stress path of Fraser River sand during rotation of $\alpha_{\sigma}$ between $-90^{\circ}$ and $+90^{\circ} \quad 87$

Fig. 5.18 Comparison of number of cycle required for liquefaction during $\alpha_{\sigma c}=0^{\circ}$

Fig. 5.19 Excess pore pressure and maximum shear strain response of Fraser River sand during cyclic loading with smooth rotation of $\alpha_{\sigma}$ from $\alpha_{\sigma c}=0^{\circ}$

Fig. 5.20 Shear stress on the horizontal plan during smooth rotation of $\alpha_{\sigma}$ from $\alpha_{\sigma c}=30^{\circ}$

Fig. 5.21 Comparison of number of cycle required for liquefaction during $\alpha_{\sigma c}=30^{\circ}$ 92

Fig. 5.22 Excess pore pressure and maximum shear strain response of Fraser River sand 
during cyclic loading with smooth rotation of $\alpha_{\sigma}$ from $\alpha_{\sigma c}=30^{\circ}$

Fig. 5.23 Shear stress on the horizontal plan during smooth rotation of $\alpha_{\sigma}$ from $\alpha_{\sigma c}=45^{\circ} 95$

Fig. 5.24 Comparison of number of cycle required for liquefaction during $\alpha_{\sigma c}=45^{\circ} \quad 96$

Fig. 5.25 Excess pore pressure and maximum shear strain response of Fraser River sand during cyclic loading with smooth rotation of $\alpha_{\sigma}$ from $\alpha_{\sigma c}=45^{\circ}$

Fig. 5.26 Shear stress on the horizontal plan during smooth rotation of $\alpha_{\sigma}$ from $\alpha_{\sigma c}=60^{\circ} \quad 98$

Fig. 5.27 Comparison of number of cycle required for liquefaction during $\alpha_{\sigma c}=60^{\circ}$

Fig. 5.28 Excess pore pressure and maximum shear strain response of Fraser River sand during cyclic loading with smooth rotation of $\alpha_{\sigma}$ from $\alpha_{\sigma c}=60^{\circ}$

Fig. 5.29 Shear stress on the horizontal plan during smooth rotation of $\alpha_{\sigma}$ from $\alpha_{\sigma c}=90^{\circ} \quad 101$

Fig. 5.30 Comparison of number of cycle required for liquefaction during $\alpha_{\sigma c}=90^{\circ}$

Fig. 5.31 Excess pore pressure and maximum shear strain response of Fraser River sand during cyclic loading with smooth rotation of $\alpha_{\sigma}$ from $\alpha_{\sigma c}=90^{\circ}$

Fig. 5.32 Shear stress on the horizontal plan during no rotation for different $\alpha_{\sigma c}$ 105

Fig. 5.33 Comparison of excess pore pressure ratio during $\Delta \alpha_{\sigma}=0^{\circ}$

Fig. 5.34 Shear stress on the horizontal plan during $\Delta \alpha_{\sigma}=30^{\circ}$ rotation for different $\alpha_{\sigma c}$ 108

Fig. 5.35 Comparison of number of cycle required for liquefaction during $\Delta \alpha_{\sigma}=30^{\circ}$ 108

Fig. 5.36 Shear stress on the horizontal plan during $\Delta \alpha_{\sigma}=45^{\circ}$ rotation for different $\alpha_{\sigma c}$ 110

Fig. 5.37 Comparison of number of cycle required for liquefaction during $\Delta \alpha_{\sigma}=45^{\circ}$ 
Fig. 5.38 Shear stress on the horizontal plan during $\Delta \alpha_{\sigma}=60^{\circ}$ rotation for different $\alpha_{\sigma c}$

Fig. 5.39 Comparison of number of cycle required for liquefaction during $\Delta \alpha_{\sigma}=60^{\circ}$

Fig. 5.40 Shear stress on the horizontal plan during $\Delta \alpha_{\sigma}=90^{\circ}$ rotation for different $\alpha_{\sigma c}$

Fig. 5.41 Comparison of number of cycle required for liquefaction during $\Delta \alpha_{\sigma}=90^{\circ}$

Fig. 6.1 Excess pore pressure induced during isotropic increment of

principal stresses for $k_{c}=1.5$

Fig. 6.2 Excess pore pressure induced during isotropic increment of

principal stresses for $k_{c}=2.0$

Fig. 6.3 Excess pore pressure induced during isotropic increment of

principal stresses for $k_{c}=3.0$

Fig. 6.4 Excess pore pressure induced during isotropic increment of

principal stresses for $k_{c}=1.3$

Fig. 6.5 Excess pore pressure induced during isotropic increment of

principal stresses for $k_{c}=1.5$

Fig. 6.6 Excess pore pressure induced during isotropic increment of

principal stresses for $k_{c}=1.8$

Fig. 6.7 Excess pore pressure induced during isotropic increment of

principal stresses for $k_{c}=2.0$

Fig. 6.8 Variation of pore water pressure with magnitude of deviatoric stress 
Fig. 6.9 Induced excess pore pressure during anisotropic principal stress increment

Fig. 6.10(a) Variation of pore water pressure parameters during anisotropic principal stress increment

Fig. 6.10(b) Variation of pore water pressure parameters during anisotropic principal stress increment

Fig. 6.11 Influence of principal stress direction on pore pressure generation for $k_{c}=1.0,1.5,2.0$

Fig. 6.12 Influence of principal stress direction on pore pressure parameter for $k_{c}=1.0,1.5,2.0$

Fig. A.1 Stress path of Fraser River sand during rotation of $\alpha_{\sigma}=30^{\circ} \pm 0^{\circ}=30_{-}^{+0}$

Fig. A.2 Stress path of Fraser River sand during rotation of $\alpha_{\sigma}=30^{\circ} \pm 30^{\circ}$

Fig. A.3 Stress path of Fraser River sand during rotation of $\alpha_{\sigma}=30^{\circ} \pm 45^{\circ}$

Fig. A.4 Stress path of Fraser River sand during rotation of $\alpha_{\sigma}=30^{\circ} \pm 60^{\circ}$

Fig. A.5 Stress path of Fraser River sand during rotation of $\alpha_{\sigma}=30^{\circ} \pm 90^{\circ}$

Fig. A.6 Stress path of Fraser River sand during rotation of $\alpha_{\sigma}=45^{\circ} \pm 0^{\circ}$

Fig. A.7 Stress path of Fraser River sand during rotation of $\alpha_{\sigma}=45^{\circ} \pm 30^{\circ}$

Fig. A.8 Stress path of Fraser River sand during rotation of $\alpha_{\sigma}=45^{\circ} \pm 45^{\circ}$

Fig. A.9 Stress path of Fraser River sand during rotation of $\alpha_{\sigma}=45^{\circ} \pm 60^{\circ}$

Fig. A.10 Stress path of Fraser River sand during rotation of $\alpha_{\sigma}=45^{\circ} \pm 90^{\circ}$

Fig. A.11 Stress path of Fraser River sand during rotation of $\alpha_{\sigma}=60^{\circ} \pm 0^{\circ}$ 
Fig. A.12 Stress path of Fraser River sand during rotation of $\alpha_{\sigma}=60^{\circ} \pm 30^{\circ}$

Fig. A.13 Stress path of Fraser River sand during rotation of $\alpha_{\sigma}=60^{\circ} \pm 45^{\circ}$

Fig. A.14 Stress path of Fraser River sand during rotation of $\alpha_{\sigma}=60^{\circ} \pm 60^{\circ}$

Fig. A.15 Stress path of Fraser River sand during rotation of $\alpha_{\sigma}=60^{\circ} \pm 90^{\circ}$

Fig. A.16 Stress path of Fraser River sand during rotation of $\alpha_{\sigma}=90^{\circ} \pm 0^{\circ}$

Fig. A.17 Stress path of Fraser River sand during rotation of $\alpha_{\sigma}=90^{\circ} \pm 30^{\circ}$

Fig. A.18 Stress path of Fraser River sand during rotation of $\alpha_{\sigma}=90^{\circ} \pm 45^{\circ}$

Fig. A.19 Stress path of Fraser River sand during rotation of $\alpha_{\sigma}=90^{\circ} \pm 60^{\circ}$

Fig. A.20 Stress path of Fraser River sand during rotation of $\alpha_{\sigma}=90^{\circ} \pm 90^{\circ}$

Fig. B.1 Excess pore pressure and maximum shear strain response of

Fraser River sand during cyclic loading with $\Delta \alpha_{\sigma}=0^{\circ}$

Fig. B.2 Excess pore pressure and maximum shear strain response of

Fraser River sand during cyclic loading with $\Delta \alpha_{\sigma}=30^{\circ}$

Fig. B.3 Excess pore pressure and maximum shear strain response of

Fraser River sand during cyclic loading with $\Delta \alpha_{\sigma}=45^{\circ}$

Fig. B.4 Excess pore pressure and maximum shear strain response of

Fraser River sand during cyclic loading with $\Delta \alpha_{\sigma}=60^{\circ}$

Fig. B.5 Excess pore pressure and maximum shear strain response of

Fraser River sand during cyclic loading with $\Delta \alpha_{\sigma}=90^{\circ}$ 


\section{LIST OF TABLES}

Table 2.1 Typical values of $A$ at failure (Braja M. Das 2008) 28

Table 2.2 Values of $A_{f}$ for normally consolidated clays (After Kenney 1959) 29

Table 5.1 Initial and loading states of tests conducted under Monotonic loading 66

Table 5.2 Initial and loading states of tests conducted under cyclic loading 67

Table 6.1 Initial and loading state of tests conducted under different

total stress path for Skempton's “ B” value test

Table 6.2 Initial and loading state of tests conducted under different

total stress path for Skempton's " $A$ " and Henkel's " $a$ " value test

Table 6.3 Comparison of pore pressure generation under hydrostatic and $3 \mathrm{D}$ initial stress conditions 


\section{LIST OF SYMBOLS}

A Skempton's pore pressure parameter

$A / D \quad$ Analog to Digital

a Henkel's pore pressure parameter

$C_{c} \quad$ Coefficient of curvature

$C_{u} \quad$ Uniformity coefficient

CSR Cyclic Stress Ratio

$D / A \quad$ Digital to Analog

DPT Differential Pressure Transducer

DPVC Digital Pressure/Volume Controller

DSC Directional Shear Cell

$D_{50} \quad$ Average particle size, $\mathrm{mm}$

$D_{r} \quad$ Relative density

$D_{r c} \quad$ Relative density at end of consolidation

$F_{z} \quad$ Vertical load

$H \quad$ Height of specimen

HCT Hollow Cylinder Torsional device

$k_{c}=\sigma_{1 c}^{\prime} / \sigma_{3 c}^{\prime}$, Effective stress ratio at the end of consolidation

$k=\sigma_{1}^{\prime} / \sigma_{3}^{\prime}$, Effective stress ratio

LVDT Linear Variable Displacement Transducer

MSC Multi-channel Signal Conditioner 
$N \quad$ Number of cycles

$P_{e} \quad$ Outer chamber pressure

$P_{i} \quad$ Inner chamber pressure

PT Phase Transformation

QSS Quasi Steady State

$R=\sigma_{1}^{\prime} / \sigma_{3}^{\prime}$, stress ratio

$R \quad$ Radius

$R_{e} \quad$ Outer radius of specimen

$R_{i} \quad$ Inner radius of specimen

$S B S \quad$ State Boundary Surface

SS Steady State

TC Triaxial Compression

TE Triaxial Extension

$T_{h} \quad$ Torque applied on the horizontal plane

$b_{\sigma}=\left(\sigma_{2}-\sigma_{3}\right) /\left(\sigma_{1}-\sigma_{3}\right)$, Intermediate principal stress parameter

$b_{\sigma c} \quad$ Intermediate principal stress parameter at the end of consolidation

$e_{\max } \quad$ Maximum void ratio

$e_{\min } \quad$ Minimum void ratio

$\alpha_{\sigma, \max }$ Maximum inclination of principal stress with vertical axis

$\alpha_{\sigma} \quad$ Inclination of major principal stress to the vertical axis

$\alpha_{\sigma c} \quad$ Inclination of major principal stress to vertical axis at the end of consolidation 
$\tau_{\text {oct }}=\frac{1}{3} \sqrt{\left(\sigma_{1}-\sigma_{2}\right)^{2}+\left(\sigma_{2}-\sigma_{3}\right)^{2}+\left(\sigma_{3}-\sigma_{1}\right)^{2}}$

$\sigma_{1} \quad$ Major principal stress

$\sigma_{2} \quad$ Intermediate principal stress

$\sigma_{3} \quad$ Minor principal stress

$\sigma_{c} \quad$ Confining stress

$\sigma_{d}=\sigma_{1}-\sigma_{3}$, deviatoric stress

$\sigma_{m} \quad$ Total mean normal stress

$\sigma_{r} \quad$ Radial stress

$\sigma_{z} \quad$ Vertical stress

$\sigma_{\theta} \quad$ Tangential stress

$\sigma_{1}^{\prime} \quad$ Effective major principal stress

$\sigma_{2}^{\prime} \quad$ Effective intermediate principal stress

$\sigma_{3}^{\prime} \quad$ Effective minor principal stress

$\sigma_{d, c y c} \quad$ Cyclic deviatoric stress

$\sigma_{m}^{\prime}=\left(\sigma_{1}^{\prime}+\sigma_{2}^{\prime}+\sigma_{3}^{\prime}\right) / 3$, Effective mean normal stress

$\sigma_{m c}^{\prime}=\left(\sigma_{1 c}^{\prime}+\sigma_{2 c}^{\prime}+\sigma_{3 c}^{\prime}\right) / 3$, Effective mean normal stress at the end of consolidation

$\Delta H \quad$ Change of height of specimen

$\Delta R_{e} \quad$ Change of outer radius of specimen

$\Delta R_{i} \quad$ Change of inner radius of specimen

$\Delta U \quad$ Excess pore pressure

$\Delta \theta \quad$ Increment of angular rotation 
$\Delta \tau_{o c t}=\left(\tau_{o c t, 1}-\tau_{o c t, 2}\right)$

$\Delta \sigma_{l} \quad$ Major component of stress increments

$\Delta \sigma_{2} \quad$ Intermediate component of stress increments

$\Delta \sigma_{3} \quad$ Minor component of stress increments

$\Delta \sigma_{d} \quad$ Increment of deviatoric stress

$\Delta \sigma_{m} \quad$ Increment of total mean normal stress

$\Delta \gamma_{\max }$ Change of maximum shear strain

$\Delta \alpha_{\varepsilon} \quad$ Increment in major principal strain direction

$\Delta \alpha_{\sigma} \quad$ Increment in major principal stress direction

$\varepsilon_{l} \quad$ Major principal strain

$\varepsilon_{2} \quad$ Intermediate principal strain

$\varepsilon_{3} \quad$ Minor principal strain

$\varepsilon_{r} \quad$ Radial strain

$\varepsilon_{z} \quad$ Axial strain

$\varepsilon_{\theta} \quad$ Tangential strain

$\tau_{z \theta} \quad$ Shear stress

$\tau_{z \theta_{\max }}$ Maximum torsional shear stress on the horizontal plane

$\gamma_{\max } \quad$ Maximum shear strain

$\gamma_{z \theta} \quad$ Torsional shear strain 


\section{ACKNOWLEDGEMENTS}

I wish to express my sincere gratitude to my supervisor Professor Siva Sivathayalan for his guidance, valuable suggestions and the encouragement throughout this research. Without his support and patience this degree would never has been possible. It has been a great pleasure to work with him.

During my research, I have earned the knowledge and experience in the advanced geotechnical laboratory in Carleton University. A research based on laboratory experiments would not have been possible without the help of laboratory technicians. I would like to acknowledge the laboratory technicians, Stanley, Pierre, Jason, and Kenneth for their great support to do my lab experiments.

I would like to take this opportunity to thank all my friends and colleagues; especially I would like to express my sincere thanks to Kourosh Khosravi and Mohammad Shahsavari for their supports and encouragements from the very first days of my research at advanced geotechnical laboratory in Carleton University.

Finally, I would like to extend very special thanks to my parents and other family members for their continuous patience, encouragement and support throughout my studies. 


\section{INTRODUCTION}

\subsection{LIQUEFACTION PHENOMENON}

The behaviour of granular soils during undrained loading is of practical interest in the field of geotechnical engineering, especially due to the likelihood of liquefaction. During undrained loading granular material tends to contract and causes an increase in pore pressure, thus decreases the effective stress. In the worst case scenario, a soil at an initially stable solid state, might lose all or most of the effective stress, and might behave as a liquid, and hence the term liquefaction.

Loose to moderately dense granular soils, such as silty sand or sands and gravels which contains impermeable sediments, are most vulnerable to liquefaction because of their low permeability. Liquefaction may also occur in granular soils of high permeability but under earthquake loading. The effective stresses decrease as a consequence of the increase in pore pressure due to shear loading, and this reduces the shear strength and softens the soil stratum which causes progressively larger and potentially catastrophic deformations. If the earthquake loading continuous even after the initiation of liquefaction it may cause excessive deformation, or even if the earthquake loading has terminated prior to the triggering of liquefaction, loss of shear strength when accompanied with the presence of static shear stresses may cause large shear deformations or flow failures on a sloping ground. Moderately dense to dense materials may cause large deformations because of the limited softening and increased shear strains but the flow failure is prevented because of the dilative tendency associate with them during shear. In moderately dense materials cyclic mobility may develop as a continuation of the limited softening if adequate number of cycles were present. Liquefaction manifests in different forms 
in-situ: Lateral spreading can occur in relatively level ground, and flow failures and large deformations in sloping grounds. Loose soils compact and undergo reconsolidation and this causes excessive settlements in ground. The process of dissipating excess pore water pressure leads to sand boils on the surface in many cases.

Liquefaction has been observed in earthquakes for many years. In fact, written records dating back hundreds of years describe earthquake effects that are now known to be associated with liquefaction (1891 Mino-Owari, 1906 San Francisco, 1940 Fukai). Nevertheless, liquefaction has been so widespread and caused extensive damage in a number of recent earthquakes that it is often associated with them. Some of those earthquakes which induced extensive damage due to liquefaction failure are 1964 Alaska, 1964 Niigata, 1989 Loma Prieta, 1995 Kobe and the 2011 Christchurch earthquakes.

\subsection{EVALUATING LIQUEFACTION SUSCEPTIBILITY}

Even though the characterization of liquefaction susceptibility is often based on empirical relationships in current practice (Seed et al. 1985), the basic understanding of this phenomenon and the effects of various factors controlling it have been derived from controlled laboratory experiments. These experimental studies have provided several insights into this phenomenon by systematically assessing the effects of individual variables. A better understanding of the mechanisms leading to soil liquefaction, and factors affecting it are critical for confident designs.

Laboratory characterization of soil behaviour is commonly carried out using conventional soil testing devices. Unfortunately conventional laboratory devices such as the triaxial and simple shear do not completely represent the loading conditions in-situ except at certain loading scenarios because loading imposed by these devices commonly ignore the effect of intermediate principal stress and principal stress direction or rotation. This is especially the case when loading 
is due to an earthquake. In addition, the nature of principal stress rotation in some of these devices may be completely different compared to that of in-situ. Therefore, reliable assessment of soil behaviour by laboratory testing requires the ability to independently control the direction of the principal stresses.

Liquefaction potential of sand depends on various state parameters including relative density, soil fabric, stress/strain history, initial stress state and loading path. The initial stress state and loading paths are commonly characterized by the following derived stress parameters

- Deviatoric stress $\sigma_{d}=\sigma_{1}-\sigma_{3}$

- Effective mean normal stress $\sigma_{m}^{\prime}=\left(\sigma_{1}^{\prime}+\sigma_{2}^{\prime}+\sigma_{3}^{\prime}\right) / 3$

- Intermediate principal stress parameter $b_{\sigma}=\left(\sigma_{2}-\sigma_{3}\right) /\left(\sigma_{1}-\sigma_{3}\right)$

- Inclination of major principal stress with vertical $\alpha_{\sigma}$

\subsection{MOTIVATION FOR THE RESEARCH USING HCT DEVICE}

Triaxial shear equipment is the most common geotechnical testing device, but it limits the intermediate principal stress parameter $b_{\sigma}$ and the inclination of major principal stress with vertical $\alpha_{\sigma}$ to specific combinations. Major principal stress $\sigma_{1}$ is always oriented along the vertical direction, and $\sigma_{2}$ and $\sigma_{3}$ are always equal to the applied cell pressure in a triaxial compression test. Thus, the intermediate principal stress parameter $b_{\sigma}=0$ and the inclination of major principal stress with vertical $\alpha_{\sigma}=0$. On the other hand during triaxial extension testing $b_{\sigma}=1$ and $\alpha_{\sigma}=90^{\circ}$ since $\sigma_{1}$ and $\sigma_{2}$ are equal and applied by the cell pressure and $\sigma_{1}$ is always oriented along the horizontal direction. During a cyclic triaxial test both intermediate principal stress parameter $b_{\sigma}$ and inclination of major principal stress with vertical $\alpha_{\sigma}$ are switched instantaneously from triaxial compression mode to triaxial extension mode or verse visa when 
cyclic shear stress amplitude exceeds static shear stress. But such a sudden jump of principal stress directions is not common during in-situ loading.

The simple shear device simulates the actual field loading conditions during an earthquake, where the response under vertically propagating shear waves is of critical concern, fairly well. But it does not permit control over the intermediate principal stress parameter, and $b_{\sigma}$ is always about 0.4 in simple shear because of the enforced plain strain condition. On the other hand the simple shear device is capable of rotating the principal stress direction smoothly during shearing but range of rotation is limited to $\pm 45^{\circ}$ on either side of the vertical direction, and the manner of rotation is uncontrollable.

Above mentioned limitations associated with conventional laboratory devices have motivated the researchers to develop more versatile devices in geotechnical testing. True triaxial, directional shear cell (DSC) and hollow cylinder torsional shear (HCT) devices represent improvements of the triaxial and simple shear devices. Even though HCT was developed many years ago it has not been used frequently because of the complexities associated with the configuration and the testing methods. The HCT devices have the capability to control four tractions such as vertical load, inner pressure, outer pressure and torque applied on the horizontal plane independently. Independent control of four surface tractions allow the control of three principal stresses and the inclination of the major principal stress facilitating soil testing under generalized loading. 


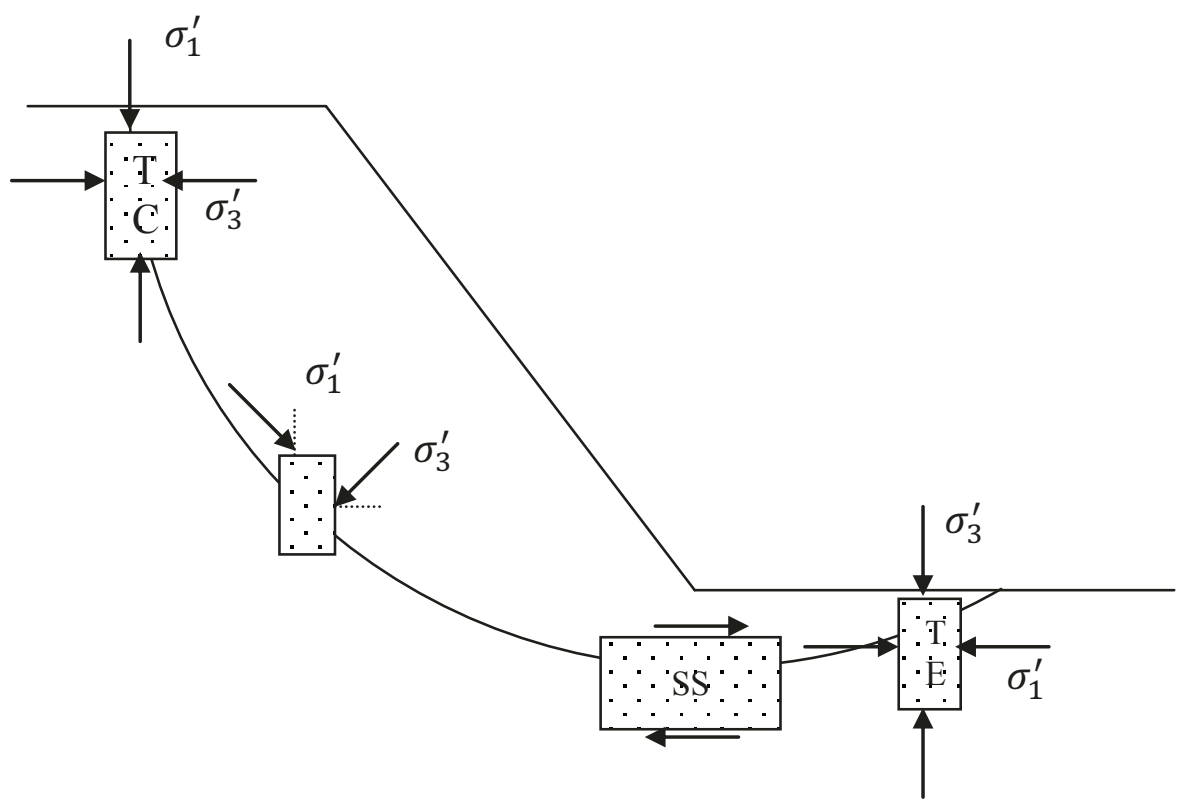

Fig. 1.1 Limiting equilibrium stress state beneath an embankment

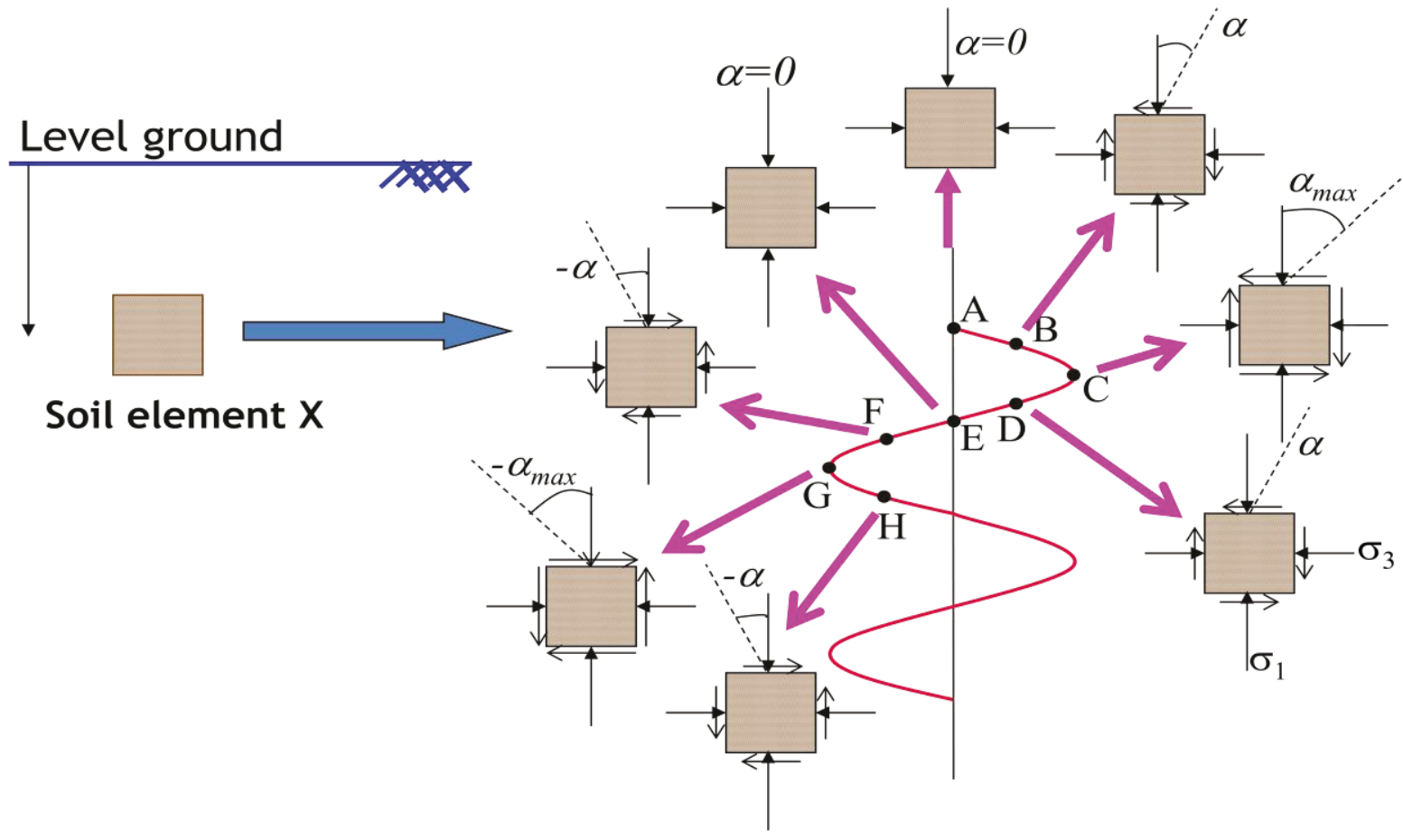

Fig. 1.2 Rotation of principal stresses in a soil element of level ground during shear wave propagation (After Logeswaran 2010) 
The stress state of a soil element mainly depends on the geometry of the soil structure and the nature of applied load. Fig 1.1 shows the direction of principal stresses on the element along a potential failure surface in an embankment. The direction of major principal stress is oriented along the vertical at the top of the failure surface and along the horizontal direction at the toe of the slope. The inclination of the major principal stress gradually changes along the failure surface as illustrated in Fig 1.1. Fig 1.2 shows the direction of principal stresses on the element in the level ground when a cyclic shear wave propagates through the soil element. During the propagation of cyclic shear wave the soil element experiences different magnitude of shear stresses. Depending on the magnitude of shear stress applied on the soil element the principal stresses act in different directions compared to the initial principal stress directions (i.e. principal stresses rotate during cyclic loading).

Few researchers (Symes et al. 1985; Uthayakumar, 1995; Sivathayalan, 2000; Logeswaran, 2010 and Manmatharajan, 2011) have studied the undrained behaviour of soils under principal stress rotations using HCT device. Those studies based on laboratory experiments were limited in the scope and several gaps exist in the current state of knowledge. Thus, further systematic studies are needed to better understand the undrained behaviour of soils subjected to principal stress rotation.

\subsection{OBJECTIVES OF THE RESEARCH}

The main objective of this research is to study the effect of the magnitude and the nature of principal stress rotation on cyclic liquefaction resistance of Fraser River sand under plane strain condition using the hollow cylinder torsional shear (HCT) device. This research studied the principal stress rotation phenomenon in a comprehensive way by considering several possible scenarios which may be expected during an earthquake in the field. Since most soil liquefaction 
related studies are from triaxial and simple shear devices, a better and generalized understanding about the effect of principal stress rotation is necessary in order apply the results from triaxial and simple shear test confidently.

As a part of the research program evaluating the behaviour of soils under generalized three dimensional loading conditions, a limited number of experiments were carried out in order to investigate the applicability of Skempton's hypothesis on pore pressure generation due to undrained loading for a more general anisotropic material at a variety of initial stress states. Originally Skempton's equation for pore pressure generation was developed from the test results of axisymmetric triaxial compression. Specifically a set of experiments were conducted on anisotropically consolidated specimens with different inclination of principal stress to analyse the behaviour of Skempton's $B$ value during a hydrostatic loading. In addition, another set of experiments were carried out on anisotropically consolidated specimen with different inclination of principal stresses to study the behaviour of Skempton's $A$ and Henkel's $a$ values during a nonhydrostatic loading.

\subsection{ORGANIZATION OF THE THESIS}

A brief description about the practical relevance with examples of liquefaction case histories are presented in this chapter. In addition this chapter noted the need for the proposed research. Following this chapter, a review of the state-of-the-art knowledge about the anisotropy of the sand, the current understanding about the undrained behaviour of sands under static and cyclic loading, and recent findings about the behaviour of sands under principal stress rotation and pore pressure generation in soils during undrained loading are discussed in chapter 2 .

Chapter 3 presents a brief description of conventional soil testing devices such as triaxial and simple shear, and a detailed discussion about the versatile hollow cylinder torsional shear 
apparatus which was used to carry out the necessary laboratory experiments for this research. Chapter 4 details the properties of the material used for the experimental work and the techniques of specimen preparation and test procedure.

Chapter 5 presents the results from the experiments, which were carried out in order to study the effect of initial principal stress direction and the principal stress rotation on the undrained behaviour of sand, which is the major part of this research program. In addition chapter 6 presents the results from the limited number of experiments used to study the pore pressure generation and behaviour of sand along different total stress paths from the specimens which were in different initial stress state. Finally chapter 7 summarizes the research findings, and presents the conclusions drawn from the comprehensive research program. 


\section{LITERATURE REVIEW}

\subsection{INTRODUCTION}

The current state-of-the-art knowledge about the undrained behaviour of sands is presented in this chapter. The emphasis has been placed on the nature of anisotropy, effects of principal stress directions and rotation, and excess pore pressure generation. Following the initial review of anisotropy, the current state of the knowledge about the behaviour of sand under static and cyclic undrained loading is discussed in the second part of the chapter.

A significant amount of research effort has focused on understanding the fundamental knowledge about undrained behaviour of sands (e.g., Ishihara et al., 1975; Castro 1969; Mohamad and Dobry, 1986; Vaid et al., 1989; Lade, 1992). Undrained state is considered as critical state in soil mechanics because it increases the excess pore water pressure which decreases the effective stress in the soil and might significantly reduce the shear strength of saturated cohesionless soils. Most of the understanding of the undrained behaviour of sands has been derived from controlled laboratory testing under different conditions. At the end of this chapter a critical review of methods available in the literature to quantify the excess pore water pressure development during the undrained loading of soils is discussed.

\subsection{ANISOTROPY IN SANDS}

Several studies have shown the existence of anisotropy in soils (Kjellman, 1936; Parkin et al., 1968; Oda, 1972; Yamada and Ishihara, 1979). The existence of anisotropy is easily revealed by direction dependent mechanical behaviour of soils. The anisotropy due to the formation process of soils has been called "inherent anisotropy" and the anisotropy formed by the shearing process has been called "induced anisotropy". 
Kjellman (1936) and Parkin et al. (1968) noticed unequal strains in all three principal directions during a hydrostatic compression. Oda (1972) explained the existence of anisotropy in sands with non-spherical particles using his observation that the preferred orientation of longitudinal axis is along the plane of deposition and the direction of contact normals. Oda (1981) also noted that anisotropy in spherical particles occurs due to the directions of contact normals.

Yamada and Ishihara (1979) and Lade and Wasif (1988) reported the existence of cross anisotropy in sands using true triaxial tests that permit measurements along all three principal directions. Stokoe et al. (1985) confirmed the existence of cross anisotropy by measuring the shear wave velocity in horizontal and vertical planes. The result of the experiments carried out by El-Sohby and Andrawes (1972) and Sayao (1989) show unequal radial and axial strains during hydrostatic compression, but equal radial and tangential strains. This is a reflection of the existence of cross anisotropy. Haruyama (1985) showed that the reconstitution technique of water pluviation leads to an inherently cross anisotropic structure using cubical triaxial tests.

In cohesionless granular soils, the spatial arrangements of solid particles progressively change during deformation. This change in soil may gradually increase the degree of anisotropy; such phenomenon has been called induced anisotropy. Oda et al. (1985) found that during shearing contact normals tends to align along the major principal direction and the longitudinal axis tends to align along the minor principal direction. Further, Oda et al. (1985) demonstrate that the degree of induced anisotropy is significantly influenced by the initial inherent anisotropy. Induced anisotropy during stage loading tests can be considered inherent anisotropy for the following stage of loading. (Wong and Arthur, 1985) 


\subsection{UNDRAINED MONOTONIC BEHAVIOUR OF SAND}

Several studies have considered the undrained response of soils to be the most critical since excess pore water pressure development during loading causes a reduction in the effective confining stresses, and thus leads to a reduction of the strength in saturated sands. This can cause excessive deformation and/or settlement. Soil liquefaction is a phenomenon, in which strength and stiffness of saturated granular soils are reduced by earthquake shaking or other means of rapid loading under undrained condition. The reduction of strength of saturated granular soil is explained by generation of significant level of excess pore water pressure during liquefaction, and the strength can potentially reach near zero or negligible levels.

Monotonic (static) undrained behaviour of saturated sands has been widely studied under triaxial loading conditions (e.g. Castro, 1969; Lee and Seed, 1980; Chern, 1985; Vaid and Thomas, 1994). Three different types of stress strain behaviour have been observed in these studies depending upon the initial state of the soil as shown in Fig 2.1. It has been noted that there is a distinct relationship between the types of behaviour and the relative density of the specimen. For identical specimens increasing the relative density from the loose state to dense state will move the soil response from type 1 to type 3 .

Loose specimens or specimens subjected to high confining stress collapse at small strain level (after reaching a peak shear stress state) and fail rapidly to mobilize large strains in Type 1. In this type of response the strength of soil specimen will increase up to a peak point and continue to decrease until it reaches a constant value. The ultimate deformation at the constant normal stress and shear stress and constant void ratio has been referred to as steady state (SS) of deformation or flow deformation. 

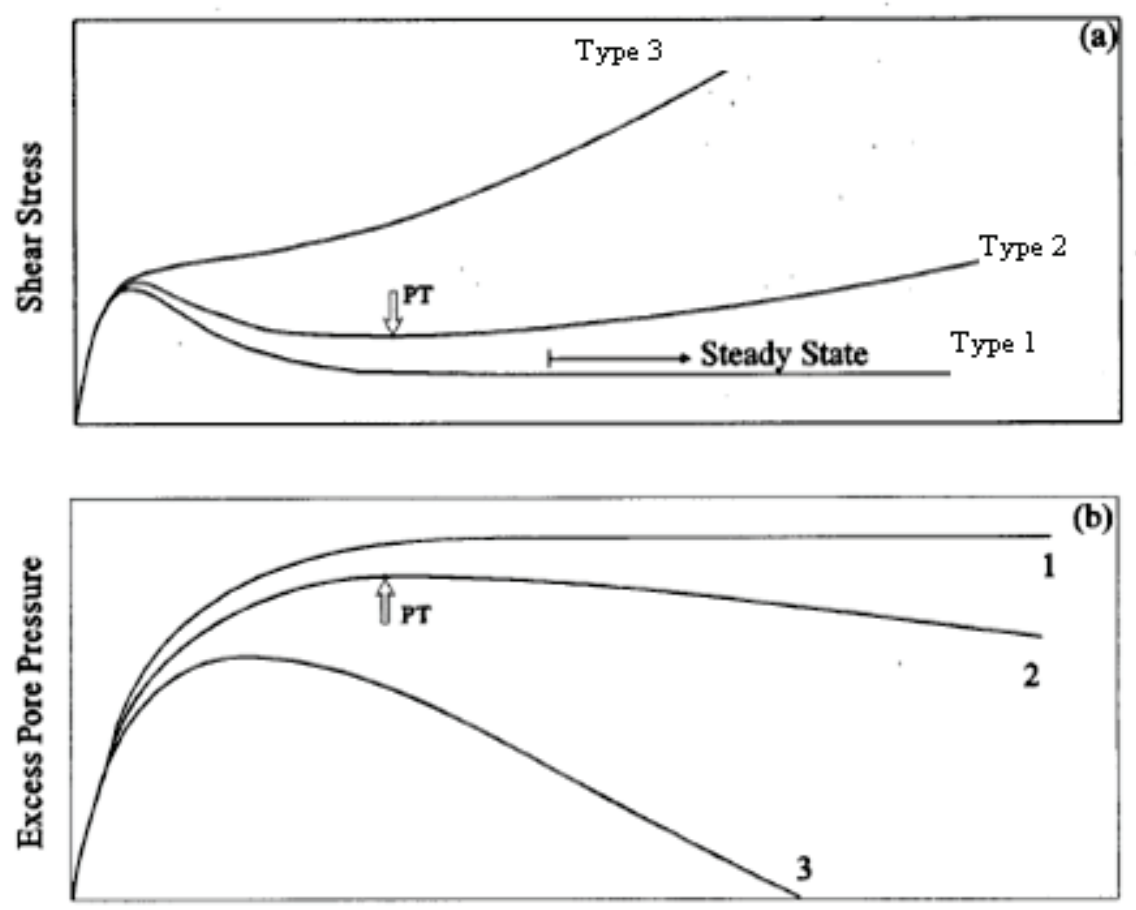

Shear Strain

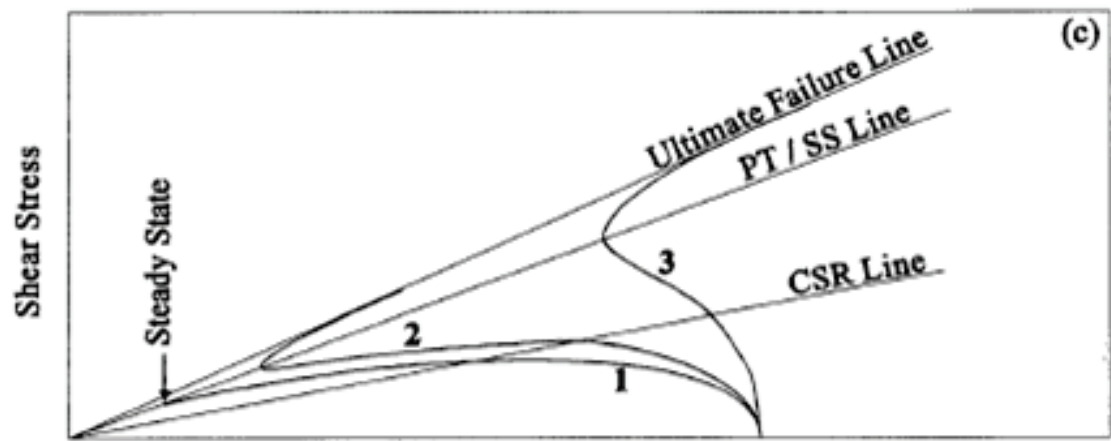

Normal Effective Stress

Fig. 2.1 Characteristic response of sand under undrained static loading (After Chern 1985)

Castro (1969) called this strain softening type of response as "liquefaction" whereas Casagrande (1975) and Seed (1979) referred as "flow liquefaction". Chern (1985) however coined the term "true liquefaction" in order to differentiate it from Type 2 response. The pore water pressure tends to increase continuously until it reaches a steady state value and remain unchanged during the flow deformation. The flow deformation occurs at a fixed point in the 
effective stress space (i.e., constant shear stress, effective normal stress and pore pressure) for a given density. The locus of such points produced by specimens with different density is called the steady state line.

The medium dense specimen initially shows similar behaviour as the loose specimen in that it reaches a peak, and then strain softens. However, the initially contractive and strain softening behaviour of soil is transformed into dilative and strain hardening behaviour upon reaching moderate levels of strain as shown in Fig. 2.1. In this type of response the strength of the soil specimen will increase until reaching a peak state and following the peak state the specimen starts to lose the strength until it reaches a minimum strength and subsequently starts to regain the strength. This kind of response has been called "limited liquefaction" by Castro (1969) and Chern (1985) and "partial liquefaction" by Lee and Seed (1967). Ishihara et al. (1975) named the minimum strength state as Quasi Steady State (QSS) since it looks like a transient steady state similar to Type 1. It has been demonstrated that QSS can be treated as a state where a limited amount of deformation occurs under constant stress and void ratio. The pore water pressure tends to increase continuously until reaches the QSS and start to decrease afterwards and that point in the stress path called as phase transformation line.

The dense specimen shows the Type 3 strain hardening response in which strength of the specimen continues to increase even though initially a small contractive behaviour is often noted. In terms of pore water pressure generation, specimen shows a positive pore water pressure during the contractive behaviour for a small strain range and thereafter large negative pore water pressures develop. So a dense specimen may shows contractive behaviour for initial small strain range and dilative behaviour at large strain level with a large shear strength, potentially even larger than the drained shear strength. 
While the relative density of the material is readily recognized to control the type of response, in reality the characteristic response of a sand depends on soil fabric, initial stress state (confining stress, shear stress) and loading mode, in addition to the relative density. It is shown that at identical initial void ratio - effective stress state, the undrained behaviour is profoundly affected by the fabric that ensues upon sample reconstitution (Vaid and Sivathayalan, 2000). At a given loading mode, an increase in relative density may transform the undrained response of sand from type 1 to type 3 (Vaid and Thomas, 1995; Vaid and Sivathayalan, 1996; Uthayakumar and Vaid, 1998). Increasing the confining stress while keeping other initial state variables constant may induce more contractive response (Vaid and Thomas, 1995; Vaid and Sivathayalan, 1996; Uthayakumar and Vaid, 1998). An increase in the static shear stress at constant confining stress promotes strain softening, despite a potential increase in relative density (Bishop 1966; Vaid and Thomas 1995; and Vaid and Sivathayalan, 2000).

The loading mode is generally characterized by the inclination of the major principal stress $\left(\alpha_{\sigma}\right)$ with specimen axis or the deposition direction. The TC and TE responses of loosest deposited Fraser River sand subjected to a range of confining stress has shown that the TC response is dilative, even for the loosest accessible state. In contrast, the TE response is strain softening over a range of initial states (Vaid and Sivathayalan, 2000). Uthayakumar and Vaid (1998) showed that at given initial state, a gradual transformation of the response of Fraser River sand occurs from dilative to strain softening, reflecting the influence of $\alpha_{\sigma}$ as it increase from zero to $90^{\circ}$. 


\subsection{UNDRAINED CYCLIC BEHAVIOUR OF SAND}

Cyclic loading will be induced during earthquakes and applied on in situ soils. The potential for liquefaction during cyclic loading is much higher than that during monotonic loading since only contractive soils can 'liquefy' under monotonic loading, but both contractive and dilative sands may liquefy under cyclic loading. A better understanding of the behaviour of soils under cyclic undrained loading, and factors that influence liquefaction potential is essential to safeguard against liquefaction failures. There are similarities in the behaviour of soils during cyclic and monotonic loading (Castro et al., 1982 and Vaid et al., 2000). Similar to monotonic behaviour the cyclic behaviour also mainly depends on initial stress state (confining stress level and initial static shear stress level), relative density of soil specimen (void ratio), loading mode and fabric. Under otherwise identical conditions, a denser specimen shows stronger response compared to a looser specimen. The intensity and the duration of the cyclic loading are also important, since a progressively increasing number of cycles (in larger earthquakes) may increase the amount of excess pore pressure generated, and lead to liquefaction, whereas a smaller earthquake may not produce sufficient number of load cycles to trigger liquefaction.

Vaid and Chern (1985) have demonstrated different strain development mechanisms occur during cyclic loading depending on the initial state of sand. These include true liquefaction and limited liquefaction which are similar to the monotonic undrained behaviour and cyclic mobility with or without transient state of zero effective stress. Unlike liquefaction, cyclic mobility causes strains which are limited in magnitude, but progressively increasing with load cycles. The initial development of strain is associated with stiffness degradation with cycles of loading. Because of the different mechanisms that are responsible for the strain development, the results of cyclic 
loading are generally assessed in terms of fixed level of strain developed in a given number of cycles.

Figure 2.2 shows the stress-strain, strain development and effective stress path plot for loose sand under cyclic loading which shows contractive strain softening behaviour leading to true liquefaction. During true liquefaction a sudden increase in pore pressure is accompanied by sudden development of large axial strain. This type of sand generates flow failure type of deformation under cyclic loading. The effective stress states at the instant at which the flow failure type of deformation begins lies on a line called as Critical Stress Ratio (CSR) line by Vaid and Chern (1985).

The development of strain during cyclic loading is minimal until the soil reaches the CSR line, once the soil reaches the CSR line soil starts to strain soften and large strain is suddenly induced in strain softening materials at a nearly constant stress state. This is analogous to the steady state under monotonic loading where the strength of soil remains at constant level and unlimited and unidirectional deformation will occur. However, in cyclic loading unloading pulses in the earthquake time history may lead to large oscillations. 

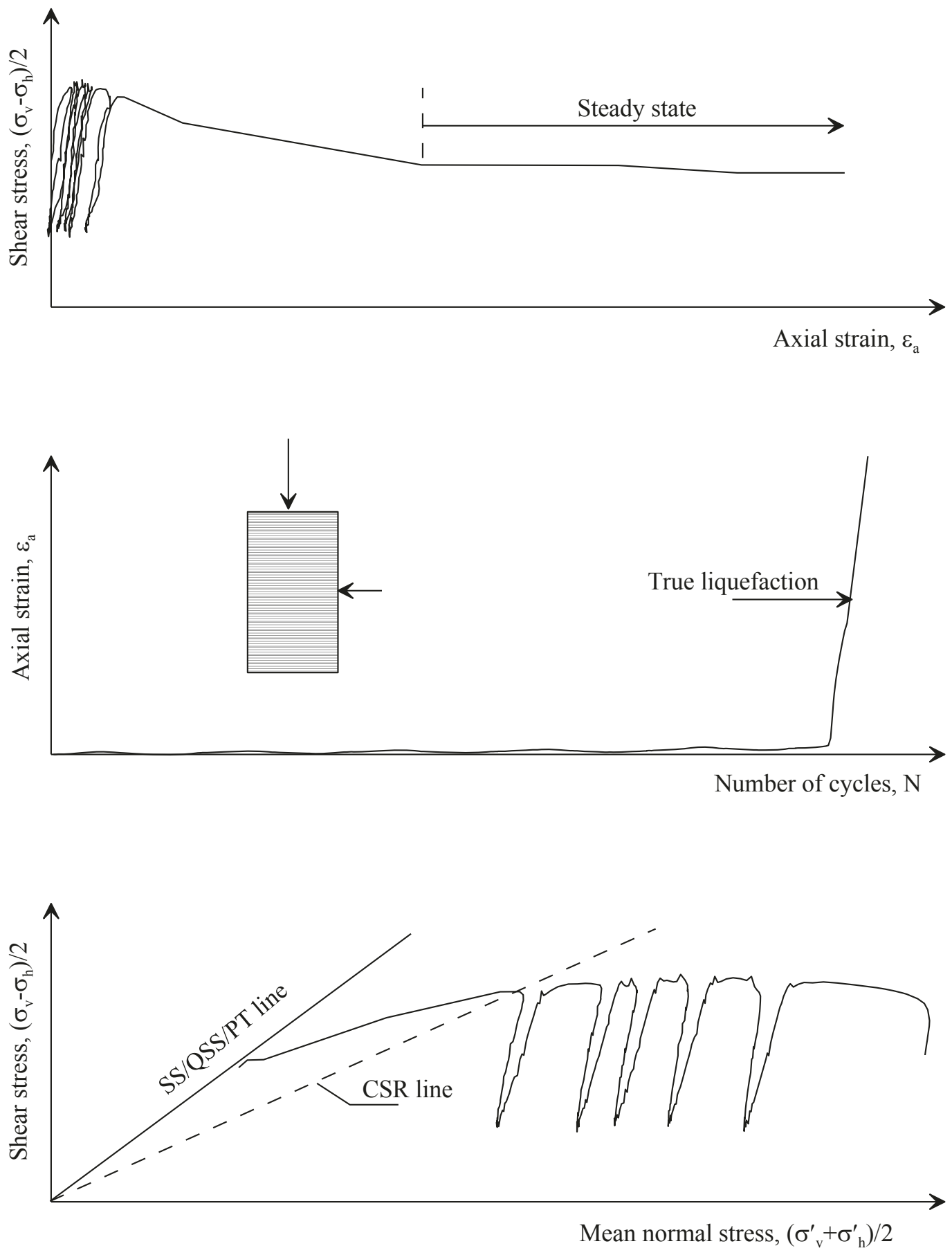

Fig. 2.2 True liquefaction of sand during cyclic undrained shear (After Vaid and Chern 1985) 
Figure 2.3 shows the stress-strain, strain development and effective stress path plot for medium dense sand which shows limited liquefaction behaviour under cyclic loading similar to type 2 response under static loading. Limited liquefaction may be followed by cyclic mobility if additional stress cycles are applied (Vaid and Chern, 1985). Vaid and Chern, (1985) have demonstrated that the true liquefaction and limited liquefaction can occur in cyclic loading only if the cyclic amplitude is larger than the minimum undrained shear strength of material at a given state.

Dense sand shows strain hardening behaviour and a possibility for liquefaction due to cyclic mobility if adequate cycles are applied. Cyclic mobility may be accompanied with a transient state of zero effective stress (Fig. 2.4) that occurs when the amplitude of cyclic shear stress is higher than the magnitude of static shear stress (or in other words, if stress reversal occurs during cyclic loading). A transient state of zero effective stress is necessary to develop large deformations. The unloading pulse upon reaching the phase transformation (PT) state usually generates large excess pore water pressure, and the effective stress state reaches zero soon thereafter. On the other hand cyclic mobility may also be accompanied without a transient state of zero effective stress (Fig. 2.5) that occurs if the magnitude of static shear stress is higher than the amplitude of cyclic shear stress and with a gradual strain development.

Seed (1979) found that strain development is small until the excess pore pressure ratio $\left(\frac{\Delta u}{\sigma \prime m c}\right)$ exceeds about $60 \%$. In contractive sands however large strains may develop without high excess pore pressure. However, a pore pressure ratio of $100 \%$ is normally pre requisite for large strain development due to cyclic mobility. 

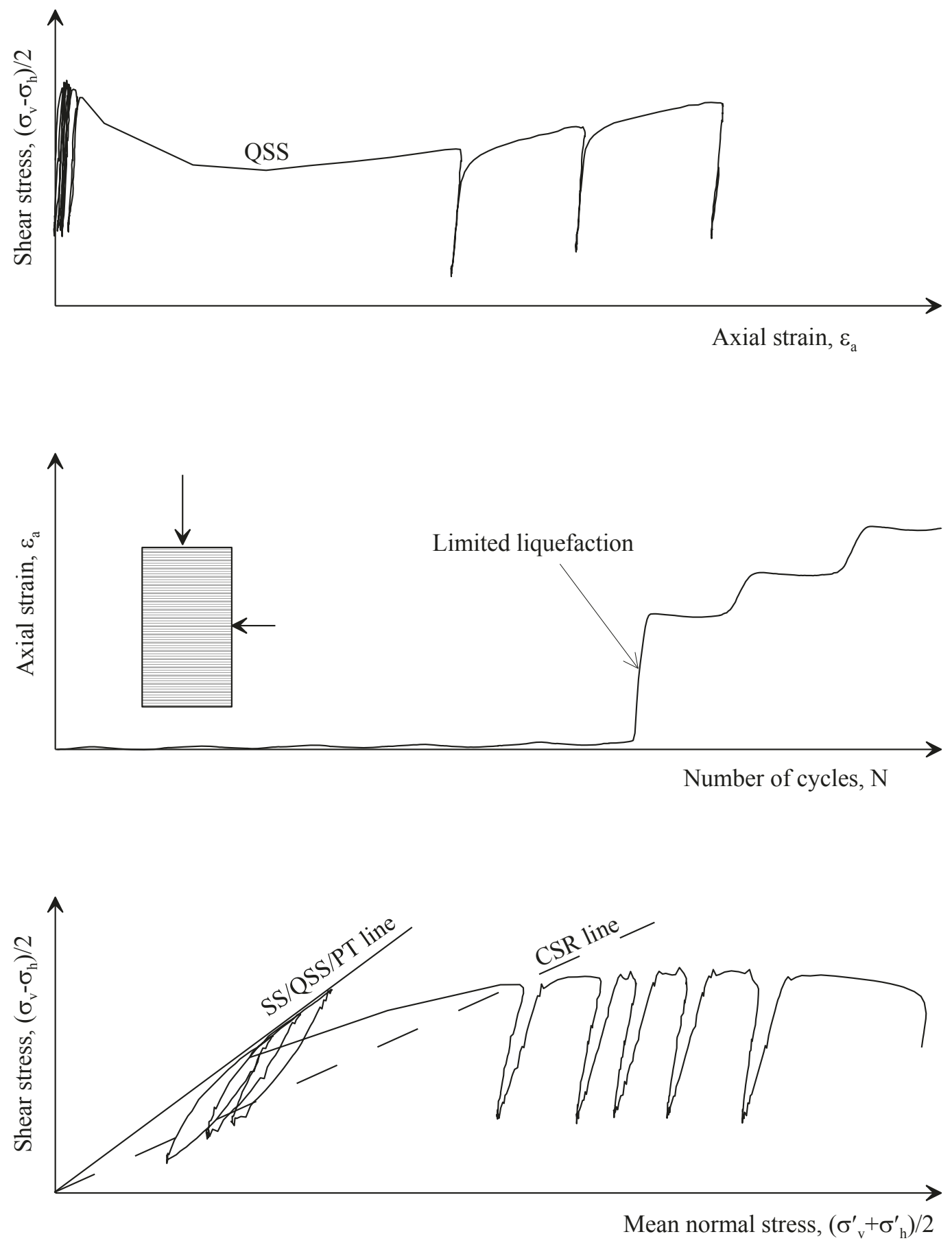

Fig. 2.3 Limited liquefaction of sand during cyclic undrained shear (After Vaid and Chern 1985) 

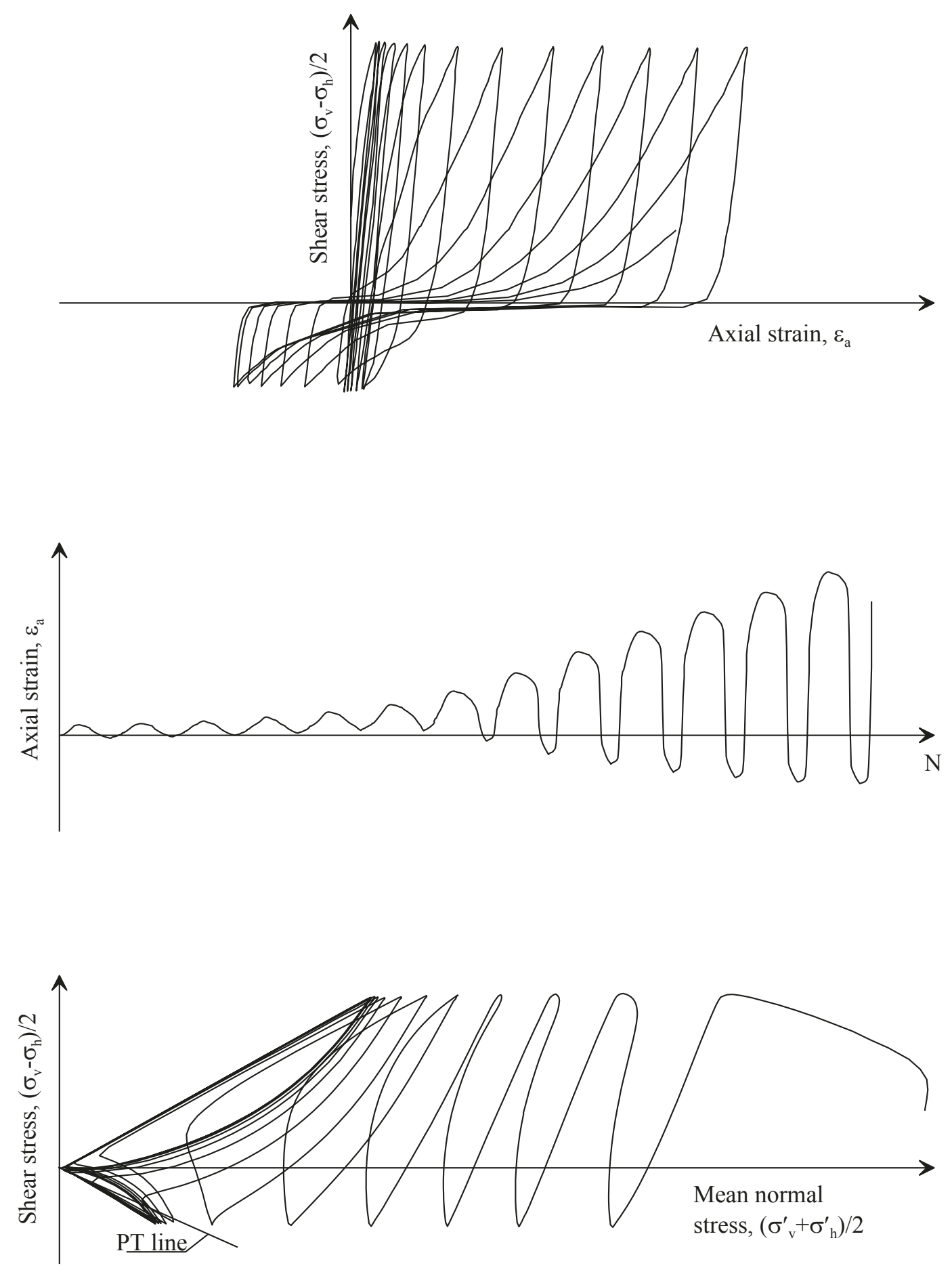

Fig. 2.4 Cyclic mobility in sand during cyclic undrained shear with transient state of zero effective stress (After Vaid and Chern 1985) 

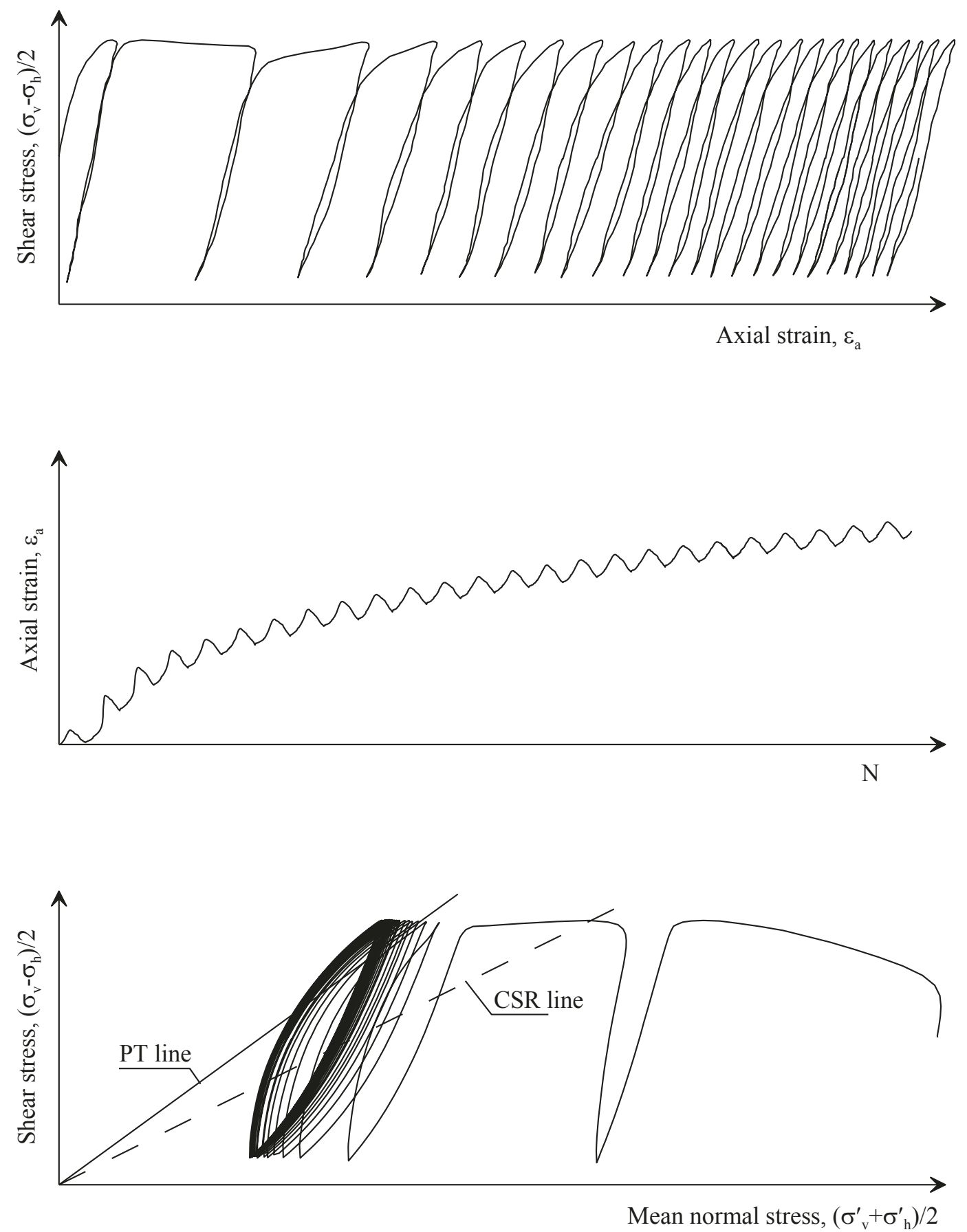

Fig. 2.5 Cyclic mobility in sand during cyclic undrained shear without transient state of zero effective stress (After Vaid and Chern 1985) 


\subsection{EFFECT OF PRINCIPAL STRESS DIRECTION}

As explained earlier, granular soils are inherently anisotropic because of particle orientation and contact normal distribution. Due to material anisotropy, the behaviour of a granular material is generally direction dependent. This inherent anisotropy causes the response of a granular soil to loading dependent on the orientation of principal stresses with reference to the depositional plane.

Broms and Casbarian (1965) conducted a study of principal stress rotation on clay under undrained condition using hollow cylinder specimens. The results showed that the continuous principal stress rotation increased the pore water pressure and decreased the friction angle at failure and consequently reduced the undrained strength of the soil.

Symes et al. (1984) investigated the anisotropy and the effects of principal stress rotation in medium-loose sand by testing it under undrained conditions using a hollow cylinder apparatus. Principal stresses have been rotated at a constant shear stress during both monotonic and cyclic loading. The experimental observations are described and then explained within a framework based on the concept of state boundary surfaces (SBS). Pore pressures are shown to be generated by rotation of principal stress directions at constant shear stress and their accumulation during cyclic principal stress rotation can lead to failure.

Symes et al. (1988) investigated the effects of principal stress rotation on the drained behaviour of saturated medium-loose sand using a hollow cylinder apparatus, where the principal stress directions were rotated both at a constant shear stress and under an increasing shear stress during drained monotonic loading to failure. The experimental results showed that specimens loaded along the vertical direction exhibited higher shear strength and initial stiffness and underwent lower volumetric strain. Increasing inclination of principal stress direction reduced 
initial stiffness and shear strength and increased induced volumetric strain. At constant shear stress level, rotation of principal stress direction generated volume contraction irrespective of the sense of rotation but the magnitude of volume contraction was depends on the sense of rotation.

Miura et al. (1986) carried out a series of drained tests on a dense anisotropic sand using hollow cylinder torsional shear apparatus. The experimental results showed that the shear deformation of sand due to rotation of principal stress axis is not negligible as compared with that with fixed principal stress axis. Despite the fact that the three principal stresses were kept constant, the specimen accumulated contractive strains due to the rotation of principal stress axis. Even though the specimen contracts under the rotational conditions, under the irrotational stress condition specimen expands irrespective of the direction of the principal stress axes within the same stress domain. Above fact suggests that under undrained condition the resistance against liquefaction will be reduced by rotation of principal stress axes. The manifestation of this under undrained loading leading to liquefaction at constant principal stresses has indeed been demonstrated by Sivathayalan and Vaid (2002).

Sayao (1989) studied the drained behaviour under rotation of principal stresses in while keeping $\sigma_{m}^{\prime}, R, b_{\sigma}$ constant, where effective principal stress ratio $R=\frac{\sigma_{1}^{\prime}}{\sigma_{3}^{\prime}}$. The results show that the continuous principal stress rotation at constant principal stress magnitudes induce progressive accumulation of both volumetric contractions and shear distortions. Further, principal stress rotation may be noted to induce contractive volume changes regardless of whether $\alpha_{\sigma}$ is increased or decreased on either side of vertical direction. Volumetric strain tends to be more significant for increasing phase of $\alpha_{\sigma}$ than for the decreasing phase with the largest contraction being associated with the first time increasing rotation phase. Contractive strains become progressively smaller with further rotation regardless of direction, which imply hardening effects 
due to previous rotations. Both volumetric strain and maximum shear strain induced due to rotation decrease progressively with increase in relative density. Much larger volumetric and shear strains were induced in sand at higher confining stresses for a given rotation angle. For a given $\alpha_{\sigma}$ both induced volumetric strain and shear strain increased with the level of R. It was noted that the value of $b_{\sigma}$ does not appear to influence the strain response of sand to principal stress rotation in a major way.

Uthayakumar (1995) studied the static undrained behaviour of sand using a HCT device. The results show that undrained response of loose water-deposited sand is highly dependent on the loading direction, and sand which is dilative when the major principal stress coincides with the deposition direction may transform into contractive as the direction of major principal stress changes from the deposition direction to the bedding plan direction.

Yoshimini et al. (1998) have pointed out that the direction of the principal stress is key factor in the undrained behaviour of the sand. They carried out undrained monotonic loading triaxial compression and extension tests on Toyoura sand and observed different shear behaviour during triaxial compression and extension tests (the shear behaviour was more contractive and softer in triaxial extension than in triaxial compression). To clarify these effects a series of undrained shear tests on Toyoura sand was performed by means of hollow cylinder torsional shear apparatus and the results showed for any density, a larger $\alpha_{\sigma}$ value, namely larger inclination of $\sigma_{1}$ from the vertical generated greater excess pore water pressure.

Sivathayalan and Vaid (2002) report an experimental study that evaluated the dependence of the monotonic undrained behaviour of sand on the orientation of principal stresses with respect to the bedding plane and the initial stress state. The result shows that the rotation of principal stress directions alone at constant deviator stress causes excess pore pressure 
development and hence an increase in the effective stress ratio, resulting in shear deformation. Further they have concluded that the susceptibility of sand to liquefaction due to stress rotation increases as the direction of the major principal stress approaches the orientation of the bedding plane.

Yang et al. (2007) conducted an experimental investigation in an automated hollow cylinder apparatus into the undrained anisotropic behaviour of saturated sand in rotational shear, which is defined as a class of non-proportional loading with a continuous rotation of the principal stress directions but at a constant deviatoric stress. The result shows that the soil specimens were weakened by the build-up of pore water pressure during rotational shearing under undrained conditions. The degree of weakening was dependent on the density of the specimen, the magnitude of the deviatoric stress and, particularly, the intermediate principal stress parameter $b_{\sigma}$.

Li et al. (2009) showed that a lower stiffness and a more contractive behaviour are observed when the loading direction deviates further away from the deposition direction or the previous loading direction. They have studied the monotonic behaviour of granular materials under different loading in directions, and conducted experiments where the major principal stress varied from vertical to horizontal at $15^{\circ}$ intervals.

Logeswaran (2010) and Manmatharajan (2011) conducted cyclic torsional shear tests using the hollow cylinder device to assess the influence of the rotation of principal stress on liquefaction potential. The tests were carried out on hydrostatically consolidated specimens and major principal stress was allowed to rotate symmetrically about the specimen axis. Both smooth and jump rotations were imposed on the loosest deposited sand, subjected to a given level of cyclic stress ratio. The test results clearly indicate that liquefaction resistance is dependent on the 
magnitude of stress rotation, and the lowest resistance was measured when the principal stresses were inclined at $45^{\circ}$ (jump rotation tests with fixed directions) or rotated between about $45^{\circ}$ to $60^{\circ}$ (smooth rotation tests with continuous rotation) to vertical. This behaviour was explained by the orientation of the plane of maximum shear stress, which is along the horizontal bedding planes during the $45^{\circ}$ rotation leads to the weakest response. Both of these researchers concluded that even though the direction of major principal stress is important, and its alignment along the bedding planes leads to softer response, but its influence is secondary compared to the orientation of the plane of maximum shear stresses with respect to the bedding plane.

\subsection{PORE PRESSURE GENERATION IN SATURATED SOILS}

Considerable attention has been directed towards the prediction and measurement of excess pore water pressure due to changes in the state of stress under undrained conditions. Pore water pressure generation in a saturated soil during undrained loading significantly influences the strength of the soil, and it is mainly related to the tendency for volume change during the application of shear stress.

Contractive volume change tendencies give rise to positive, and dilative tendencies lead to negative excess pore water pressure generation. The amount of excess pore pressure generated during undrained loading has been quantified in terms of increments of principal stresses. Skempton (1954), based on axisymmetric triaxial tests on hydrostatically consolidated specimens, proposed a relationship to quantify the amount of excess pore pressure generated in a triaxial compression test in terms of major $\Delta \sigma_{1}$ and minor $\Delta \sigma_{3}$ principal stress increments as

$$
\Delta u=B\left[\Delta \sigma_{3}+A\left(\Delta \sigma_{1}-\Delta \sigma_{3}\right)\right]
$$


Skempton called $A$ and $B$ as pore pressure coefficients, and in current practice these are widely known as Skempton's pore pressure parameters. Skempton's $B$ parameter depends on the degree of saturation, and $A$ accounts for the shear induced excess pore pressure.

When the loading involves no shear stresses, i.e., for a hydrostatic stress increment with $\Delta \sigma_{1}=\Delta \sigma_{3}(=\Delta \sigma)$, the equation simplifies to $\Delta u=B \Delta \sigma$. Considering elastic compression of the isotropic soil skeleton and pore fluid Skempton (1954) has shown that

$$
B=\frac{1}{1+n\left(C_{F} / C_{S k}\right)}
$$

where, $n$ is the porosity, and $C_{F}, C_{S k}$ are the compressibility of the pore fluid and soil skeleton respectively. Compressibility of the pore fluid is function of the degree of saturation, and in fully water saturated soils $\left(S_{r}=1\right)$, the value of $C_{F}$ is negligible compared to $C_{S k}$ and thus $B=1$. $C_{S k}$ in very stiff soils may also be relatively low compared to soft soils, and thus the Skempton's $B$ value may fall just below 1 in such soils even if they are fully saturated. The value of $B$ in a given soil decreases rapidly toward zero when degree of saturation decreases.

The coefficient $A$ has been determined experimentally by several researchers for different types of soils in the literature at the failure state. Technically, $A$ value can be determined as a function of shear stress level, but the interest is often in the $A$ value at failure. This is sometimes termed $A_{f}$ in the literature, but often the term $A$ refers to the $A$ value at failure. Skempton has demonstrated that $A=\frac{1}{3}$ for isotropic elastic materials, but in natural soils $A$ can take a range of values.

Application of Skempton's pore pressure equation without regard to principal stress rotation and intermediate principal stress has led to some confusion in the literature. Originally, Skempton's equation was based only on axisymmetric triaxial compression. It was reviewed by Skempton (1961) and expressed in terms of absolute values of principal stress difference. 
Attempts have been made by Terzaghi et al. (1996) to generalize Skempton's approach to account for three dimensional loading conditions as shown in the equation below, but there have not been sufficient experimental investigations to confidently determine parameters $A_{1}$ and $A_{2}$ to date, and the practice still relies on the original formulation of Skempton.

$$
\Delta u=B\left[\Delta \sigma_{3}+A_{1}\left(\Delta \sigma_{1}-\Delta \sigma_{3}\right)+A_{2}\left(\Delta \sigma_{2}-\Delta \sigma_{3}\right)\right]
$$

The loading mode or the stress path to failure highly influences the magnitude of $A$ for a given soil other than the composition and structure of the soil. Hence the magnitude and the behaviour of $A$ cannot be generalized in order to quantify the pore water pressure generation during undrained shearing to use in effective stress stability analysis. However Table 2.1 gives typical range of $A$ values at failure for various soils.

Table 2.1 Typical values of $A$ at failure (Das 2008)

\begin{tabular}{|c|c|}
\hline Type of soil & $A$ \\
\hline Clay with high sensitivity & $0.75-1.5$ \\
Normally consolidated clay & $0.5-1.0$ \\
Over consolidated clay & $-0.5-0$ \\
Compacted sandy clay & $0.5-0.75$ \\
\hline
\end{tabular}

The contact materials which trends to develop positive shear induced pore water pressure during undrained shear loading give positive values for the Skempton's pore pressure parameter $A$. Loose soils tend to collapse during the application of shear stress when they subjected to high consolidation pressure, hence they yield relatively high Skempton's pore pressure parameter $A$. Moderately over consolidated clays (OCR approximately 4 to 8) may either contract or expands during undrained shear loading and generally yield approximately zero shear induced pore water pressure, thus Skempton's pore pressure parameter $A$ would be close to zero at failure. Heavily 
over consolidated clays and dense sands show dilative tendency during undrained sharing especially when they are subjected to low effective confining pressure and develop high negative shear induced pore water pressure, thus their Skempton's pore pressure parameter $A$ is negative and rarely less than about -0.3. (Terzaghi et al., 1996). Table 2.2 gives the typical values of $A$ at failure $\left(=A_{\mathrm{f}}\right)$ for some normally consolidated clayey soils.

Table 2.2 Values of $A_{f}$ for normally consolidated clays (After Kenney 1959)

\begin{tabular}{|c|c|c|c|c|c|}
\hline Clay & Type & Liquid limit & $\begin{array}{c}\text { Plasticity } \\
\text { index }\end{array}$ & Sensitivity & $A_{f}$ \\
\hline \multicolumn{6}{|l|}{ Natural soils } \\
\hline Toyen & Marine & 47 & 25 & 8 & $1.48-1.50$ \\
\hline Drammen & Marine & 36 & 16 & 4 & $1.2-2.4$ \\
\hline Saco River & Marine & 46 & 17 & 10 & 0.95 \\
\hline Boston & Marine & - & - & - & 0.85 \\
\hline Bersimis & Estuarine & 39 & 18 & 6 & 0.63 \\
\hline Chew stoke & Alluvial & 28 & 10 & - & 0.59 \\
\hline Kapuskasing & Lacustrine & 39 & 23 & 4 & 0.46 \\
\hline DecomposedTalus & Residual & 50 & 18 & 1 & 0.29 \\
\hline St.Catherines & Till & 49 & 28 & 3 & 0.26 \\
\hline \multicolumn{6}{|l|}{ Remolded soils } \\
\hline London & Marine & 78 & 52 & 1 & 0.97 \\
\hline Weald & Marine & 43 & 25 & 1 & 0.95 \\
\hline Beauharnois & Till & 44 & 24 & 1 & 0.73 \\
\hline Boston & Marine & 48 & 24 & 1 & 0.69 \\
\hline Beauharnois & Estuarine & 70 & 42 & 1 & 0.65 \\
\hline Bersimis & Estuarine & 33 & 13 & 1 & 0.38 \\
\hline
\end{tabular}

Henkel (1960) has suggested an equation in terms of principal stress increments to express the excess pore pressure generation in 3-D problems. The equation was based on the stress invariants 
expressed by the sum of square of the difference of principal stress increments and the sum of all principal stress increments.

$$
\Delta u=\left[\frac{\Delta \sigma_{1}+\Delta \sigma_{2}+\Delta \sigma_{3}}{3}+\frac{a}{3} \sqrt{\left(\Delta \sigma_{1}-\Delta \sigma_{2}\right)^{2}+\left(\Delta \sigma_{2}-\Delta \sigma_{3}\right)^{2}+\left(\Delta \sigma_{3}-\Delta \sigma_{1}\right)^{2}}\right]
$$

Here, $\Delta \sigma_{1}, \Delta \sigma_{2}$ and $\Delta \sigma_{3}$ are the changes of principal stresses and $a$ is pore pressure parameter depending on soil type, consolidation history, and stress state. Later Henkel and Wade (1966) have revised the equation and proposed a new equation in terms of pre and post shear principal stresses to encounter shear induced excess pore pressure.

$$
\Delta u=\left[\frac{\Delta \sigma_{1}+\Delta \sigma_{2}+\Delta \sigma_{3}}{3}+\frac{a}{3} \Delta \sqrt{\left(\sigma_{1}-\sigma_{2}\right)^{2}+\left(\sigma_{2}-\sigma_{3}\right)^{2}+\left(\sigma_{3}-\sigma_{1}\right)^{2}}\right]
$$

Neither Skempton's nor Henkel's formulation consider the potential anisotropic characteristics of natural soils nor the effect of generalized initial stress states.

The terms $\Delta \sigma_{1}$ and $\Delta \sigma_{3}$ can be interpreted differently; either as the difference between the major (or minor) principal stress at the initial and final states, or in terms of the stress increment vector. This is not a cause for concern, if the loading remained triaxial compression, but can lead to inconsistent definitions when comparing states that represent triaxial compression and extension loading modes. As a result, inconsistent calculations of Skempton's $A$ values have been proposed in the literature (Law and Holtz, 1978). To unify the definition of $\Delta \sigma_{1}$ and $\Delta \sigma_{3}$, Law and Holtz (1978) have defined the stress increments as illustrated in Fig. 2.8. In that approach, the stress increment vectors were used, and algebraically largest increment was considered as $\Delta \sigma_{1}$ (major component of stress increments) and the algebraically smallest increment was considered as $\Delta \sigma_{3}$ (minor component of stress increments) regardless of the original major and minor principal stresses, so that $\Delta \sigma_{1}$ and $\Delta \sigma_{3}$ need not to be in the direction of $\sigma_{1}$ and $\sigma_{3}$ respectively. 

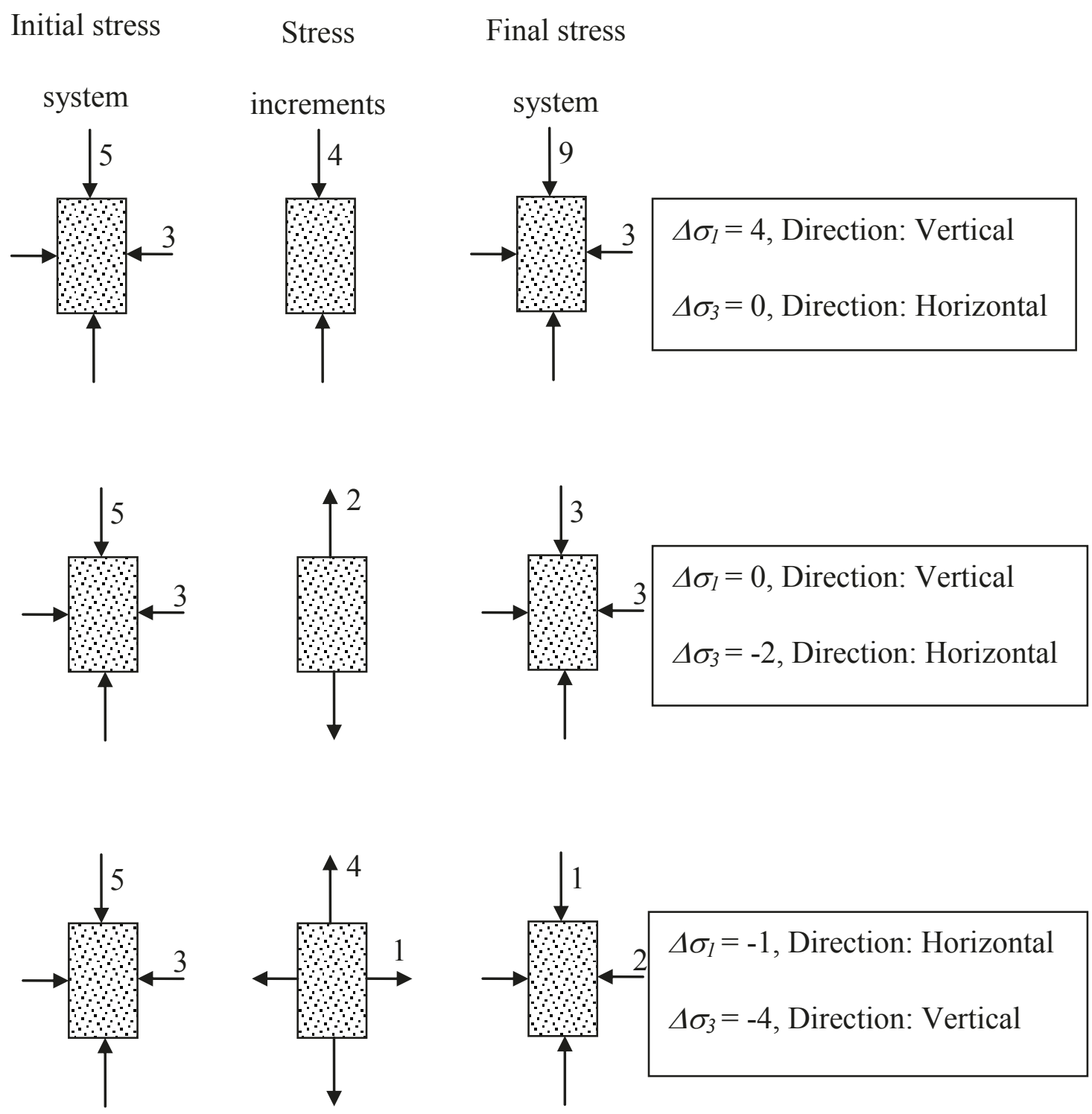

Fig.2.6 Increment of principal stresses in axisymmetric soil element during rotation of principal stress (After Law and Holtz, 1978) 


\section{LABORATORY SOIL TESTING DEVICES}

\subsection{INTRODUCTION}

Liquefaction susceptibly of soils is often assessed using field test data with empirical correlations, or from laboratory experiments carried out using conventional soil testing devices. Depending on the significance of the project both field and laboratory test data can be used to evaluate the potential for liquefaction. In order to have a realistic estimation of the in-situ loading and response, the laboratory soil testing device should have the ability to (1) apply the initial stress state prior to the commencement of loading, and (2) follow the stress path that is anticipated in-situ. The most commonly used soil test, the triaxial test, is not capable of properly simulating in-situ stress conditions in most cases. The less frequently used simple shear, true triaxial, and directional shear represent some improvements. But, a hollow cylinder torsional shear (HCT) device is the most versatile soil testing device. Each device has its own merits and drawbacks, and no device can be used to simulate actual field conditions with six degrees of freedom. The triaxial and the simple shear are the most frequently used laboratory soil testing devices even though they are not capable of applying generalized stress state and loading paths. HCT is not commonly used because of the complexity associated with it even though it can apply generalized initial stress states and loading path, and control four independent stress/strain parameters.

This chapter presents the merits and drawbacks of the fundamental laboratory soil testing devices such as triaxial and simple shear and details the versatile HCT device since all the experiments for this research program were carried using that. 


\subsection{TRIAXIAL DEVICE}

The triaxial device is widely used to evaluate the mechanical properties of soils because the testing procedure is relatively simple compared to other tests. A cylindrical specimen which has a height to diameter ratio of two is commonly used for a triaxial test. Usually the triaxial specimen is sheared by applying a vertical load while keeping the all-around pressure constant. Triaxial compression tests are more common but triaxial extension test also occasionally carried out. The triaxial specimen is enclosed within a rubber membrane and restrained at both top and bottom using end platens that have porous stones to allow drainage of water if/when required. Two stress components, namely major principal stress $\sigma_{1}$ and minor principal stress $\sigma_{3}$ can be controlled in this test. Irrespective of whether triaxial compression or triaxial extension is carried out, principal stresses always coincide with vertical and horizontal directions. There is no predetermined failure plane in triaxial device as in the direct shear device, which always has a failure plane along horizontal plane. Instead the failure plane in a triaxial test is determined by material property.

The end restraints used to support the specimen at top and bottom causes non-uniformities along the specimen and introduces some uncertainty in the interpretation of tests results, especially at large strains. This problem was studied by many researchers (Taylor, 1948; Bishop and Green, 1965), and Lade (1982) and showed that the non-uniformities can be minimized by using an aspect ratio of at-least two. Lubricated end platens have also been used to reduce the end restraint effects (Rowe and Barden, 1964; Barden and Khayatt, 1966), but this technique is known to introduce bedding errors (Sarsby et al., 1980). 

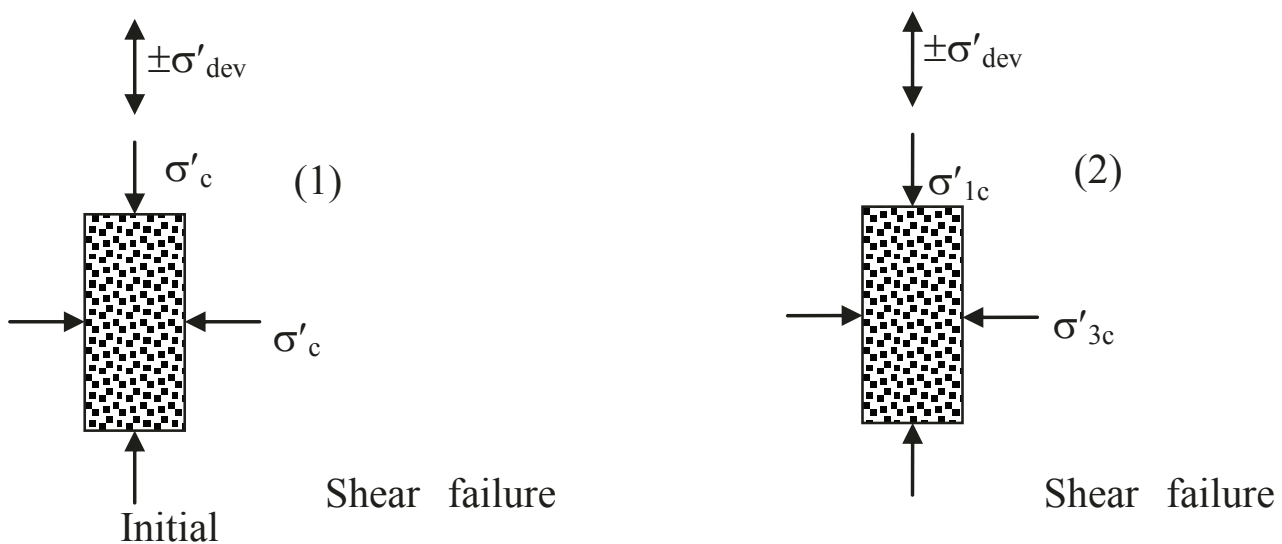

Shear failure
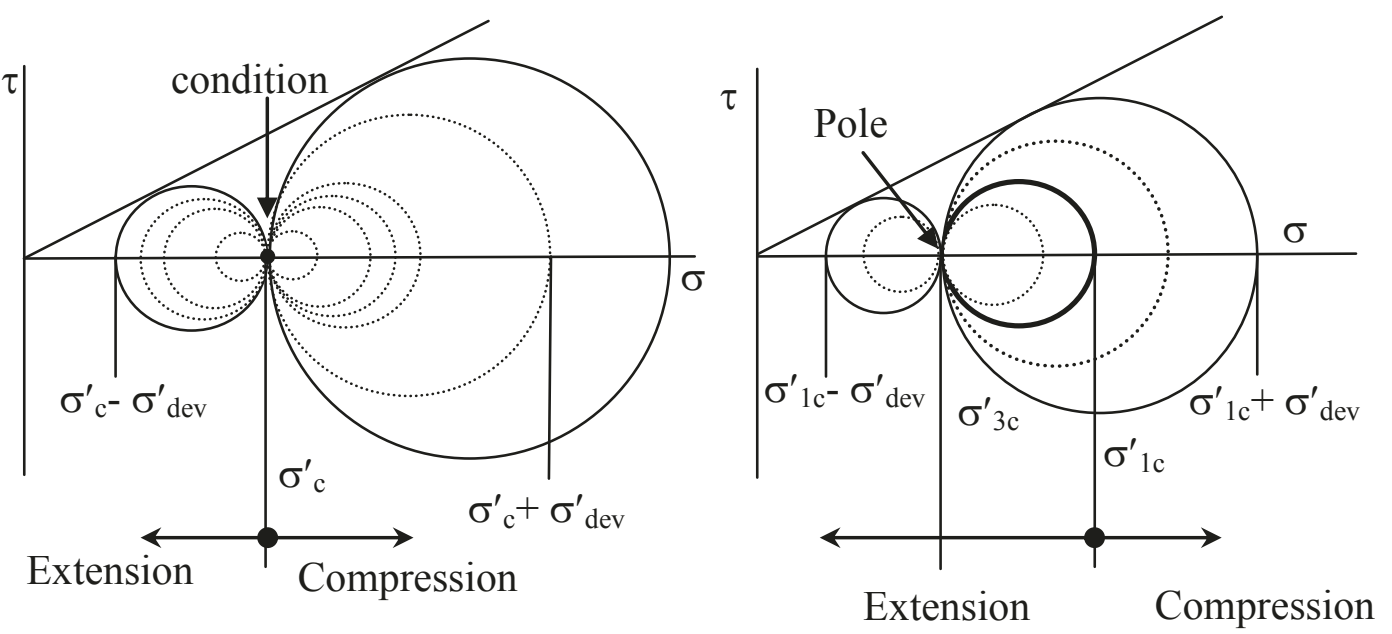

$\alpha_{\sigma}=90^{\circ} \quad \alpha_{\sigma}=0^{\circ}$

$\alpha_{\sigma}=90^{\circ}$

$\alpha_{\sigma}=0^{\circ}$

Fig 3.1 The stress representation of triaxial in Mohr's circle

The stress path during a triaxial test can be represented by Mohr's circle as shown in Fig. 3.1. Whether isotropically consolidated or anisotropically consolidated, the direction of principal stress will rotate instantaneously by $90^{\circ}$ when the axial deviatoric stress changes its direction during a cyclic triaxial test. Usually the direction of major principal stress is along vertical direction and minor principal stress is along radial direction during triaxial test, but they will switch their directions instantaneously when stress reversal occurs. In addition, a triaxial test 
always creates axisymmetric stress state, so two of three principal stresses are always equal. This kind of axisymmetric condition is not common in the actual field and the jump rotation of $90^{\circ}$ in principal stress directions cannot be expected in the field problems where principal stress rotates in a smooth manner. These properties limit the nature of stress paths that can be followed by the specimens and limits the applicability of the triaxial test for the actual field situations.

Another limitation associated with the triaxial device is the inability to control the magnitude of intermediate principal stress, which is always equal to minor principal stress $\left(\sigma_{2}=\right.$ $\left.\sigma_{3}\right)$ during triaxial compression test and equal to major principal stress $\left(\sigma_{2}=\sigma_{1}\right)$ during triaxial extension test. But in the field most of the geotechnical problems are associated with plane strain condition in which $\sigma_{2}$ may be significantly higher than $\sigma_{3}$.

In order to overcome the limitations associated with conventional triaxial devices, researchers have used the true triaxial device with different boundary conditions. In a true triaxial device, the three principal stresses are controlled independently and thus the influence of intermediate principal stress on the behaviour of soil may be assessed. However, the inclination of the principal stress or the manner of rotation of principal stresses cannot be evaluated using a true triaxial device.

\subsection{SIMPLE SHEAR DEVICE}

The simple shear device simulates the loading condition in-situ during a vertically propagating shear wave well. This ability has made this the preferred device for cyclic liquefaction studies. This device is designed to imposed normal and shear stress on the horizontal plane on anisotropically consolidated soil specimens. Typically the vertical normal stress, horizontal shear stress, axial strain and the shear strain are measured during a simple shear test. 
In practice, there are two types of simple shear devices: the Norwegian Geotechnical Institute (NGI) type and the Cambridge type. In the NGI type simple shear device, a circular specimen is laterally confined by a steel reinforced rubber membrane, and in the Cambridge type a cubical shape specimen is confined by six rigid faces.

Both types of simple shear devices simulate plane strain conditions which is the actual field condition in most geotechnical engineering problems. Stress non-uniformities arise due to the lack of complementary shear stress in a simple shear, but these can be minimized by using a small height to diameter ratio. The wire- reinforced membrane used in the NGI type devices imposes $k_{O}$ conditions during the application of the vertical load. This enables the simple shear device to appropriately simulate the in-situ stress state during consolidation. The level ground conditions are simulated by $k_{O}$ consolidation and the sloping ground stress state can be simulated by adding a shear stress on the horizontal plane during consolidation. The steel wire reinforced membrane not only provides $k_{O}$ condition during the consolidation but also restrains lateral deformation during shear which permits constant volume testing during monotonic and cyclic shear loading. In simple shear devices horizontal normal stresses are not applied externally, and are mobilized depending on the constitutive characteristics of the material. Each soil can thus be tested at its natural state without a need to explicitly specify the horizontal normal stresses. This is a major advantage compared to triaxial tests, since tests at $k_{O}$ conditions are more representative of the field.

The cyclic shear stress applied on the horizontal plane in a simple shear test simulates the actual field stress condition due to a vertically propagating shear wave. The value of simple shear in the study of soil behaviour in terms of principal stress direction and rotation is limited, but this device is useful for assessing the cyclic resistance of the soil. Since the undrained tests are 
conducted under constant volume, the pore pressure of the specimen is always atmospheric, and thus the development of excess pore pressure is actually zero. However, the drop in vertical normal stress during shearing is equal to the excess pore pressure that would have been developed (Dyvik et al. 1987).

\subsection{HOLLOW CYLINDER TORSIONAL SHEAR DEVICE}

The hollow cylinder torsional shear (HCT) device is a versatile laboratory soil testing equipment, and is used to measure the mechanical properties of soils under generalized stress state. As the name implies, a hollow cylindrical soil specimen is tested under the application of internal pressure and torque about the vertical axis, in addition to the external confining pressure and the axial load which are applied in a triaxial test. Because of the independent control over these four tractions, this test permits independent control of all three principal stresses $\left(\sigma_{1}, \sigma_{2} \& \sigma_{3}\right)$ and inclination of the major principal stress with vertical $\left(\alpha_{\sigma}\right)$.

\subsubsection{Features of the Carleton University HCT device}

The HCT device in the advanced geotechnical laboratory at Carleton University is capable of applying stress path controlled loading tests by controlling axial load, internal pressure, external pressure and torque about vertical axis on reconstituted sand specimens under monotonic and cyclic loading conditions. In addition, strain path controlled tests can be conducted by controlling the axial displacement, radial displacement, inner volume and specimen volume.

Fig. 3.2 shows a schematic view of the HCT device used for this research program. The specimen used for the testing has a height of approximately $300 \mathrm{~mm}$ with an outer diameter of $150 \mathrm{~mm}$ and an inner diameter of $100 \mathrm{~mm}$ which results in a wall thickness of $25 \mathrm{~mm}$, a cross 
sectional area of about $100 \mathrm{~cm}^{2}$ and a volume of about $3000 \mathrm{~cm}^{3}$. The reason behind the selection of such large dimensions is to reduce the level of stress non-uniformities, and improve the measurement resolutions. A $0.3 \mathrm{~mm}$ thick flexible rubber membrane is used to retain the sand specimen on which the internal and external pressure is applied laterally. The top and bottom of the specimen is restrained by annular aluminum end platens which are ribbed in the radial direction in order to ensure proper transfer of the torsional load. The vertical load is applied from the bottom of the specimen while fixing the top of the specimen, which makes the specimen preparation easer and eliminate the requirement to correct the vertical load for the weight of the load cell and the ram. The solid reaction of vertical and torsional load is ensured by the rigid steel frame attached at the bottom of the specimen. In order to ensure complete drainage of pore water during drained tests, six small porous stones are evenly placed into the built in cavities of each end platens (Fig. 3.3).

Nine parameters including four stress components, four strain components and pore pressure are measured during the test. In order to facilitate proper stress or strain path control loading the interactive use of a feedback controlled system (data acquisition and control computer) is necessary. Saturation of the system, noise level in the system and the proper functioning of transducers are frequently monitored and checked. 


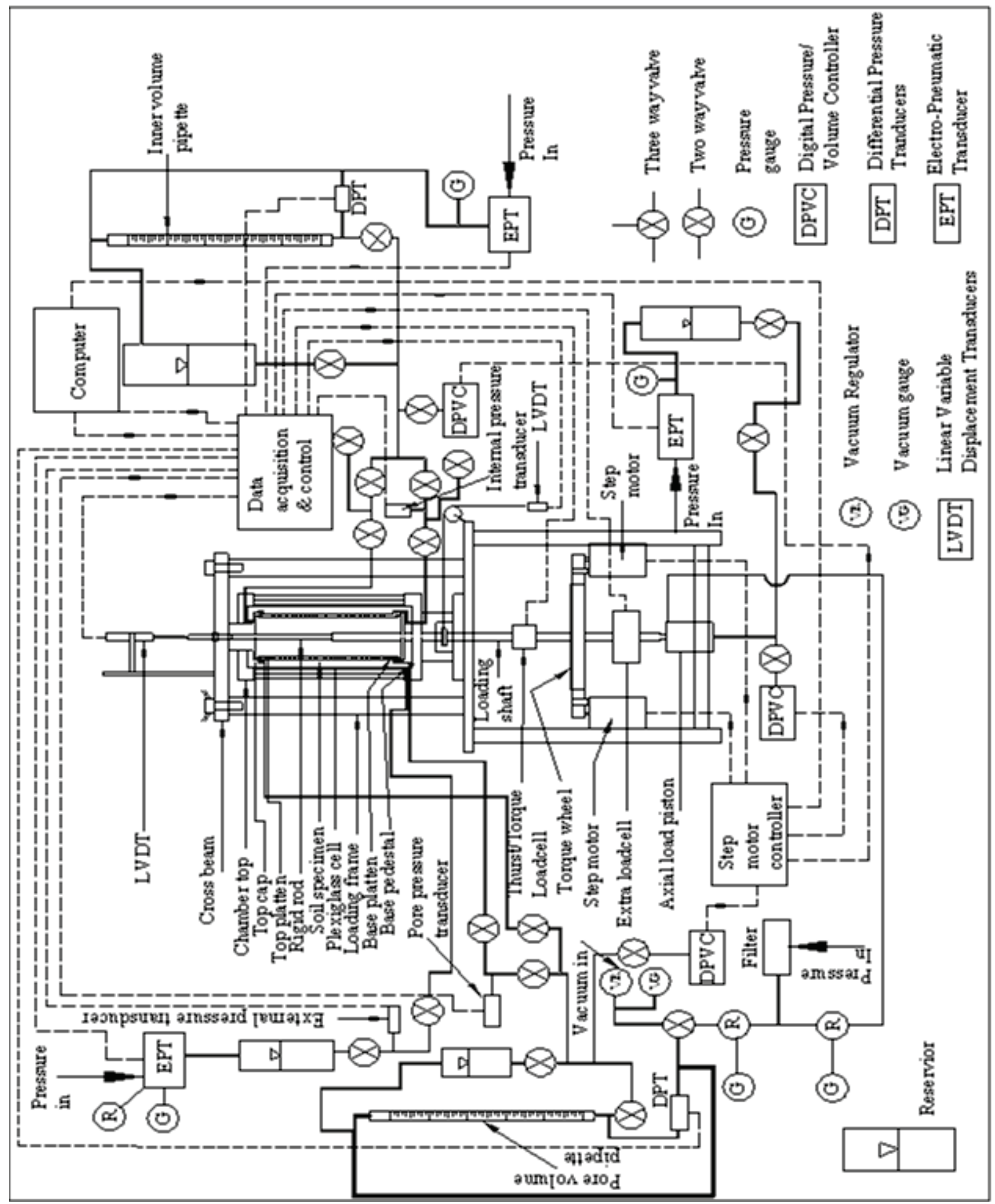

Fig. 3.2 Schematic layout of HCT device at Carleton University (After Logeswaran 2010) 


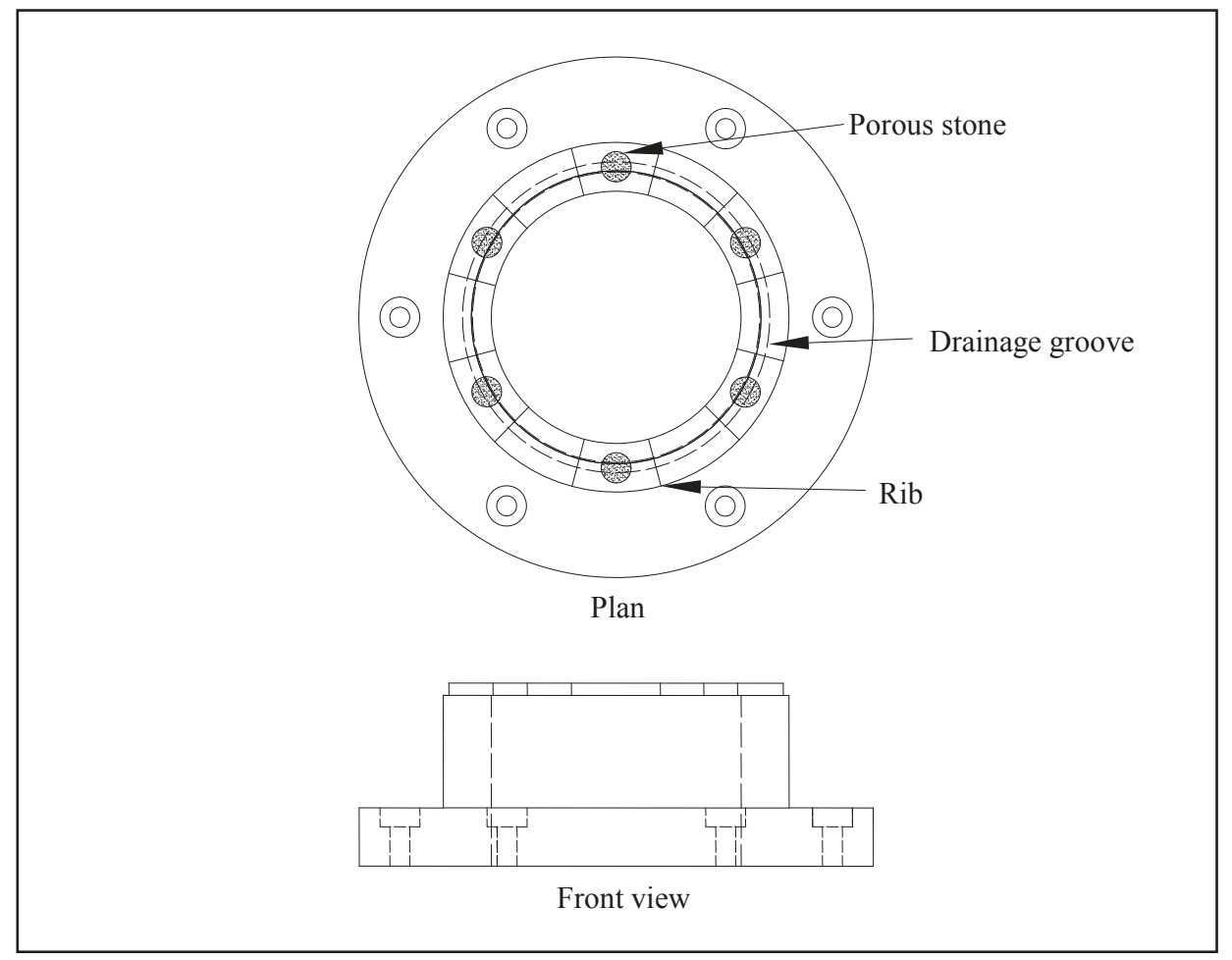

Fig. 3.3 Porous stones embedded into end platen with radial ribs (After Logeswaran 2010)

\subsubsection{Stress and strains in a HCT specimen}

The four surface tractions applied to a hollow cylinder specimen, namely the vertical force $F_{z}$, torque on the horizontal plane $T_{h}$, external pressure $P_{e}$ and internal pressure $P_{i}$ are used to determine the specific stress state of the specimen. The stress state imposed on the soil specimen is considered either in terms of the principal stresses $\sigma_{1}, \sigma_{2}$ and $\sigma_{3}$ and their inclination $\alpha_{\sigma}$, or in terms of vertical stress $\sigma_{z}$, tangential stress $\sigma_{\theta}$, radial stress $\sigma_{r}$ and shear stress on the horizontal plane $\tau_{z \theta}$. Fig. 3.4 shows the tractions applied on a soil specimen and the resulting stresses with the representation of direction of principal stresses. The strains corresponding to the direction of stresses are represented by vertical strain $\varepsilon_{z}$, tangential strain $\varepsilon_{\theta}$, radial strain $\varepsilon_{r}$ and shear strain $\gamma_{z \theta}$. These stress and strain components are used to calculate principal stress and strains and inclination of major principal stress/strain with vertical. 


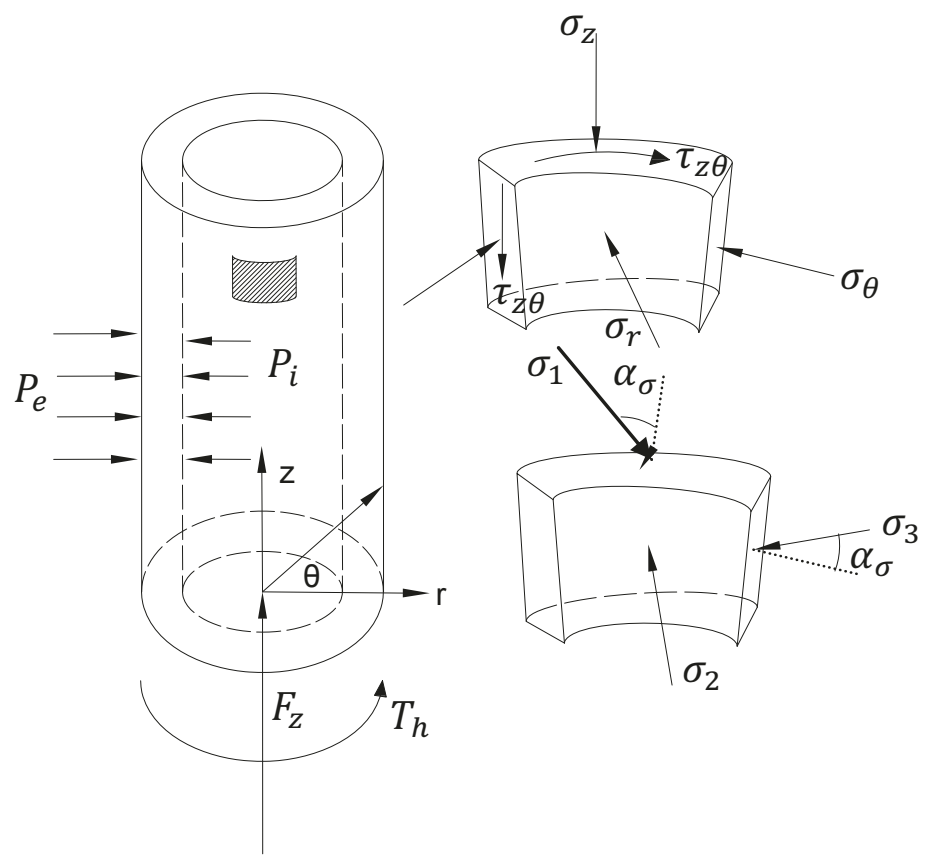

Fig. 3.4 Surface tractions and stress state of a soil element in a HCT specimen

The vertical stress $\sigma_{z}$ is directly calculated using the applied vertical load, the components of internal and external pressure applied in the vertical direction and the cross sectional area.

$\sigma_{z}=\frac{F_{z}+\pi\left(P_{e} R_{e}^{2}-P_{i} R_{i}^{2}\right)}{\pi\left(R_{e}^{2}-R_{i}^{2}\right)}$

The normal stresses $\sigma_{\theta}$, and $\sigma_{r}$ and shear stress $\tau_{z \theta}$ vary along the thickness of the specimen. So the average value of normal stresses $\sigma_{\theta}$, and $\sigma_{r}$ and shear stress $\tau_{z \theta}$ are calculated to determine the overall response of the soil. The stress state in a thick cylinder subjected to loading and deformation cannot be solved considering the equilibrium alone, and a knowledge of the constitutive relationship of the material is required to calculate the values of $\sigma_{\theta}, \sigma_{r}$ and $\tau_{z \theta}$. Even though different formulations have been proposed in the literature to determine the average values of $\sigma_{\theta}, \sigma_{r}$ and $\tau_{z \theta}$, the one equations noted below proposed by Vaid et al. (1990) considering force equilibrium and linear elastic isotropic behaviour were used in this study. 
$\sigma_{r}=\frac{\left(P_{e} R_{e}^{2}-P_{i} R_{i}^{2}\right)}{\left(R_{e}^{2}-R_{i}^{2}\right)}-\frac{2\left(P_{e}-P_{i}\right) R_{e}^{2} R_{i}^{2} \ln \left(R_{e} / R_{i}\right)}{\left(R_{e}^{2}-R_{i}^{2}\right)^{2}}$

$\sigma_{\theta}=\frac{\left(P_{e} R_{e}^{2}-P_{i} R_{i}^{2}\right)}{\left(R_{e}^{2}-R_{i}^{2}\right)}+\frac{2\left(P_{e}-P_{i}\right) R_{e}^{2} R_{i}^{2} \ln \left(R_{e} / R_{i}\right)}{\left(R_{e}^{2}-R_{i}^{2}\right)^{2}}$

$\tau_{z \theta}=\frac{4 T_{h}\left(R_{e}^{3}-R_{i}^{3}\right)}{3 \pi\left(R_{e}^{4}-R_{i}^{4}\right)\left(R_{e}^{2}-R_{i}^{2}\right)}$

where, $R_{i}$ and $R_{e}$ are internal and external radius of HCT specimen. $P_{i}$ and $P_{e}$ are the internal and external pressures and $T_{h}$ is the torque applied on the horizontal plane.

In an HCT specimen, the torsional shear stress is applied about the vertical axis or in other words in the $z \theta$ plane, and thus shear stress along the direction of $r \theta$ and $r z$ is zero. Therefore plane $r$ becomes one of the principal planes and normal stress acting on this plane is one of the principal stresses. Under most loading conditions the stress along direction $r$ is identified as intermediate principal stress $\sigma_{2}$. The magnitude of the major principal stress $\sigma_{1}$, and minor principal stress $\sigma_{3}$, and the inclination of major principal stress with vertical $\alpha_{\sigma}$ can be calculated from $\sigma_{z}, \sigma_{\theta}$ and $\tau_{z \theta}$.

$$
\begin{aligned}
& \sigma_{1}=\frac{\left(\sigma_{z}+\sigma_{\theta}\right)}{2}+\sqrt{\frac{\left(\sigma_{z}-\sigma_{\theta}\right)^{2}}{4}+\tau_{z \theta}^{2}} \\
& \sigma_{3}=\frac{\left(\sigma_{z}+\sigma_{\theta}\right)}{2}-\sqrt{\frac{\left(\sigma_{z}-\sigma_{\theta}\right)^{2}}{4}+\tau_{z \theta}^{2}} \\
& \alpha_{\sigma}=\tan ^{-1}\left(\frac{\tau_{z \theta}}{\sigma_{z}-\sigma_{3}}\right)
\end{aligned}
$$


Strain calculations assume linear variation across the wall of the specimen, which is consistent with the linear elastic response assumed in stress calculations. The following equations are used to determine the strains.

$\varepsilon_{Z}=-\frac{\Delta H}{H}$

$\varepsilon_{r}=-\left(\frac{\Delta R_{e}-\Delta R_{i}}{R_{e}-R_{i}}\right)$

$\varepsilon_{\theta}=-\left(\frac{\Delta R_{e}+\Delta R_{i}}{R_{e}+R_{i}}\right)$

$\gamma_{z \theta}=\left(\frac{2 \Delta \theta\left(R_{e}^{3}-R_{i}^{3}\right)}{3 H\left(R_{e}^{2}-R_{i}^{2}\right)}\right)$

Here, $H$ and $\Delta H$ are height, and change of height of the HCT specimen and $\Delta \theta$ is the angular displacement. $\Delta R_{e}$ and $\Delta R_{i}$ are change of external and internal radius respectively. $\Delta R_{i}$ is calculated from the measured value of $\Delta H$ and volume change of inner chamber. $\Delta R_{e}$ is calculated from the measured value of $\Delta H$ and volume change of specimen and $\Delta R_{i}$.

Similar to the stress calculations the strain components $\varepsilon_{z}, \varepsilon_{r}, \varepsilon_{\theta}$ and $\gamma_{z \theta}$ permits the determination of principal strain $\varepsilon_{1}, \varepsilon_{2}, \varepsilon_{3}$ and the inclination of major principal strain to vertical $\alpha_{\varepsilon}$

\subsubsection{Loading system of the HCT device}

The stress control loading system is simple and allows following the prescribed stress path. But, the stress controlled loading system does not allow the post peak behaviour to be captured in strain softening materials, because of the specimen - device interaction together with the development of run-away strains. So a strain control loading system is essential in order to assess 
the liquefaction potential of loose contractive sands which often strain soften during undrained loading.

The stress control loading system in the CU HCT device is activated by three ElectroPneumatic (E/P) transducers which convert an analog electrical signal input into output pressure which varies in the range of 0 to $700 \mathrm{kPa}$. The three Electro-Pneumatic transducers are connected to inner pressure chamber (Fig. 3.5), external pressure chamber (Fig. 3.6) and axial load piston (Fig. 3.7). The torque is applied by two stepper motors mounted at the bottom which apply pulses until reaching the target torque in a feedback loop controlled by the data acquisition system (Fig. 3.8).

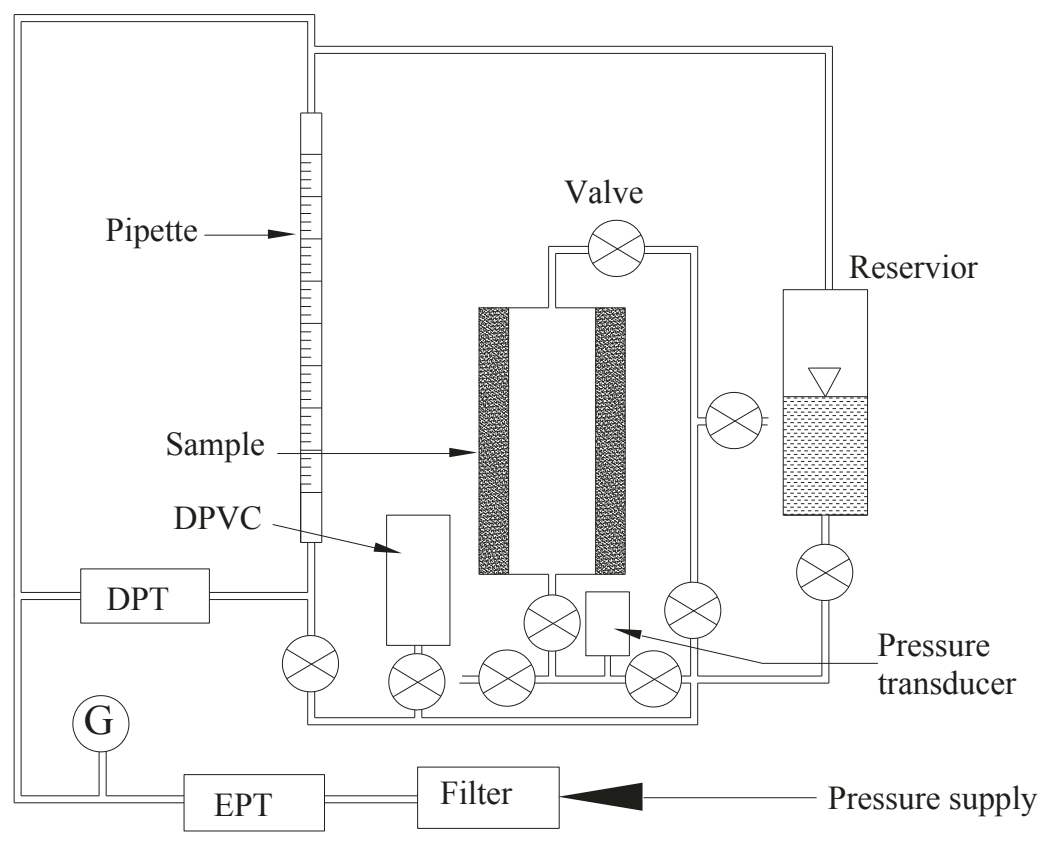

Fig. 3.5 Pressure and volume control of inner chamber (After Logeswaran 2010) 


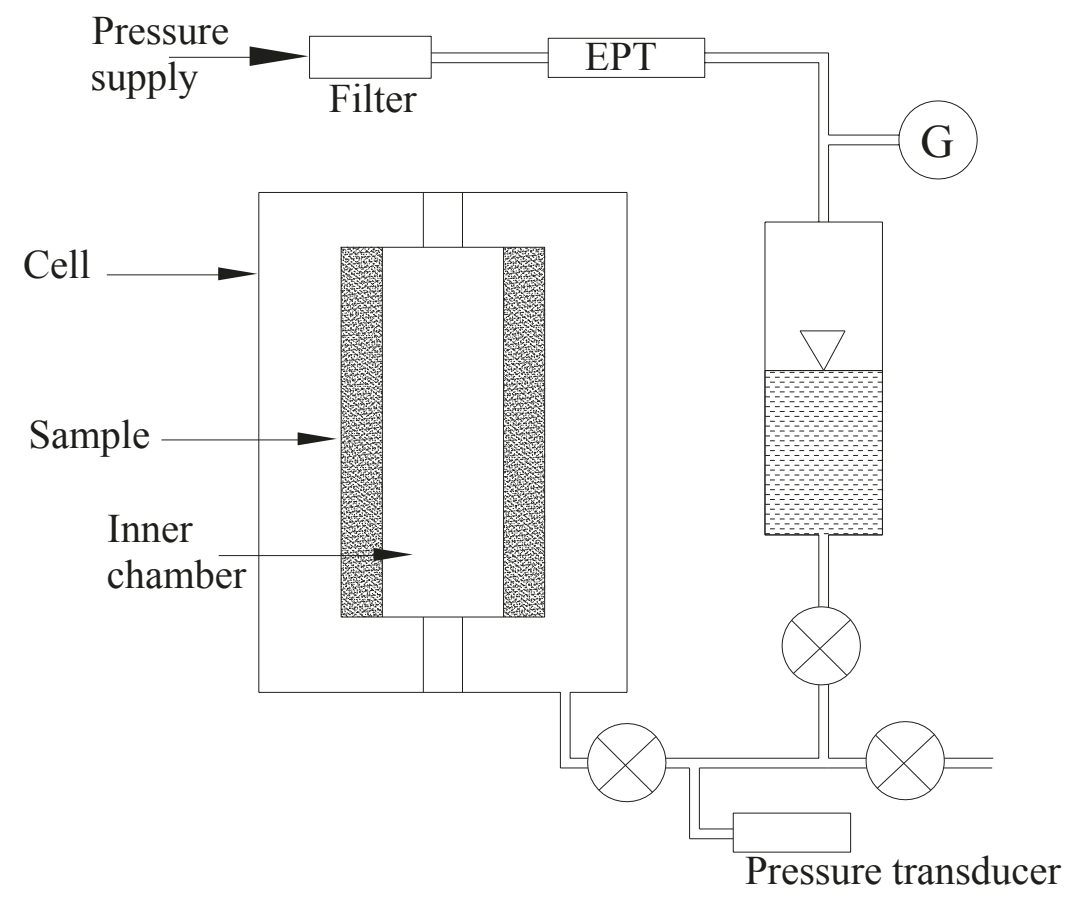

Fig. 3.6 Pressure control of external chamber (After Logeswaran 2010)

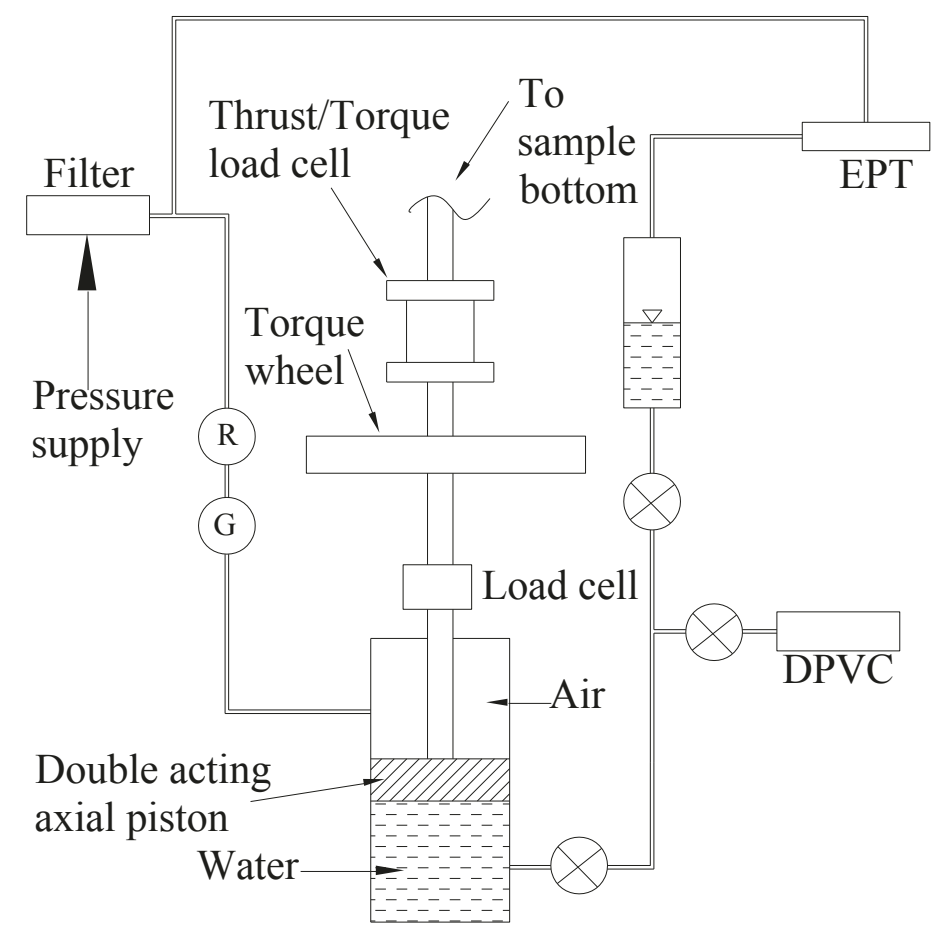

Fig. 3.7 Vertical stress and vertical displacement control (After Logeswaran 2010) 


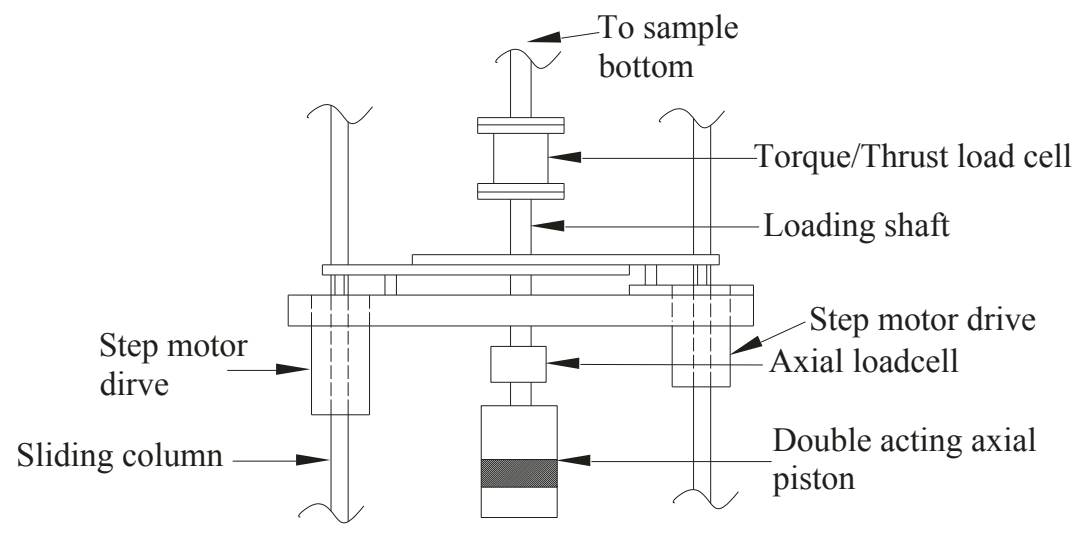

Front view

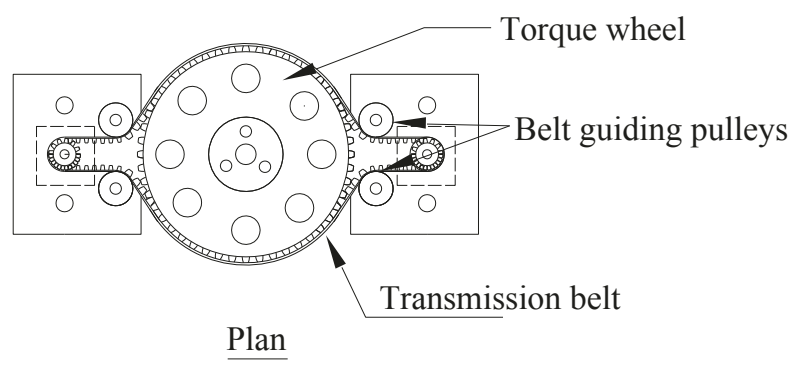

Fig. 3.8 Vertical and Torsional load applying system (After Logeswaran 2010)

Strain control loading is performed by using three Digital Pressure/Volume controllers (DPVC) and two torque motors. The three Digital Pressure/Volume controllers are connected to the vertical loading piston (Fig. 3.7), specimen volume (Fig. 3.9) and inner chamber (Fig. 3.5) and control vertical displacement, radial strain and tangential strain. Menzies (1987) pioneered the use of DPVC in soil testing which consist of a saturated water cylinder and a piston that is attached to a step motor by a ball screw. In DPVC the desired strain increment is applied by either extracting or injecting water. Since the strain increment is applied by volume control, the strain control loading system requires a fully saturated system. 


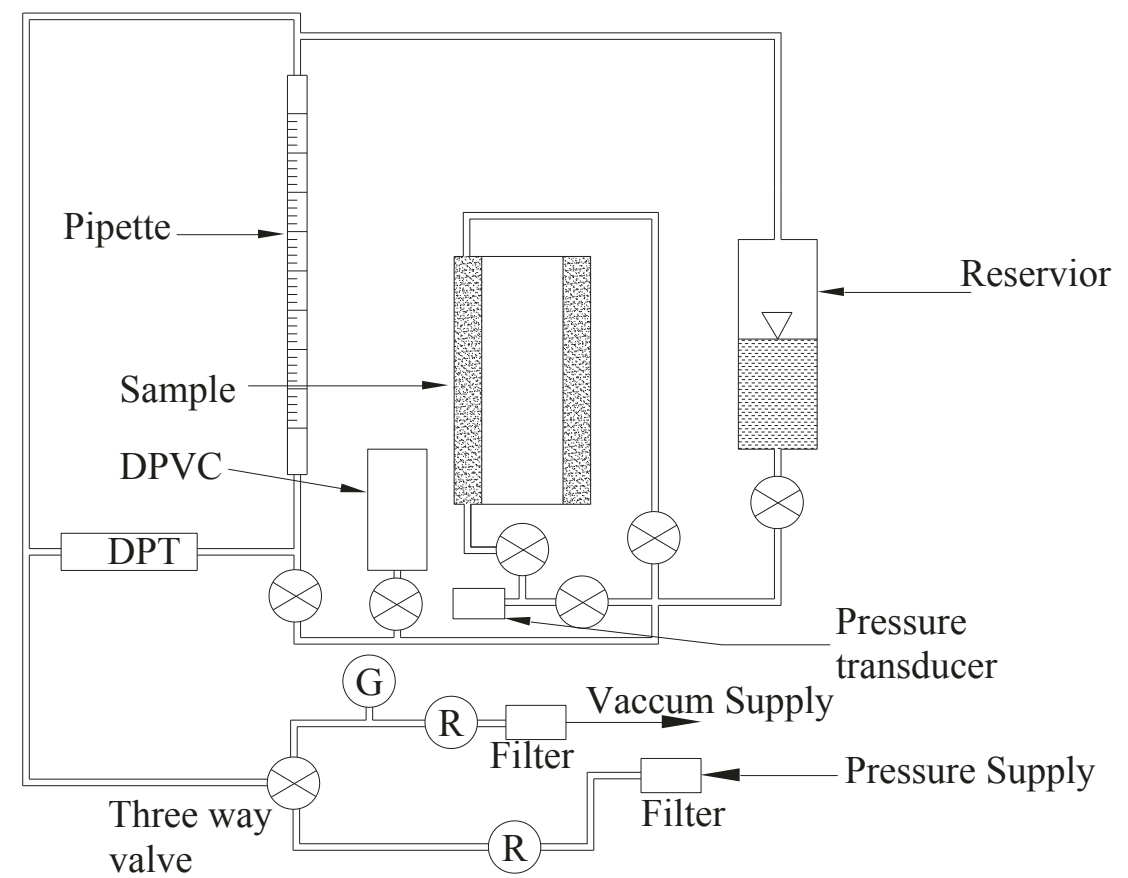

Fig. 3.9 Back pressure and specimen volume control (After Logeswaran 2010)

\subsubsection{Measuring system of the HCT device}

The CU HCT device uses nine electronic transducers to measure loads, pressures and deformation during the test. The three pressure components, namely internal chamber pressure, external chamber pressure and the pore pressure are directly measured using precision pressure transducers $(\mathrm{PT})$ which has a resolution of about $\pm 0.1 \mathrm{kPa}$. The surface traction components vertical load and torque are measured by thrust/torque load cell. The load cell has a resolution of thrust approximately $\pm 4 N$ which in terms has a vertical stress resolution in the order of $\pm 0.5 \mathrm{kPa}$. The load cell has a resolution of torque approximately $\pm 0.05 \mathrm{Nm}$ which in terms of shear stress has a resolution in the order of $\pm 0.01 \mathrm{kPa}$. The torque and thrust measurements have a negligible amount crosstalk in the load cell. In addition, the axial load is also measured 
using an extra load cell attached at the bottom of the torque loading system (Fig. 3.8). This has a resolution of approximately $\pm 1 \mathrm{~N}$ and a vertical stress resolution in the order of $\pm 0.1 \mathrm{kPa}$ (Logeswaran, 2010)

The two displacement components vertical displacement and torsional displacement are measured using Linear Variable differential Transducers (LVDT) and converted into axial and shear strain respectively. The vertical LVDT is mounted at the top of the cross beam of loading frame and the vertical displacement is directly measured by measuring the movement of the thin vertical rod that is attached to the centre of the bottom pedestal. The torsional LVDT measure the rotation of the specimen by changing the rotation as linear tangential displacement (Fig. 3.10). Both LVDTs have an accuracy level of approximately $10^{-3} \mathrm{~mm}$ which in term of axial and shear strains is better than about of $5 \times 10^{-4}$. The inner chamber volume and the sample volume changes are recorded using differential pressure transducers (DPT) which are used to calculate the radial and tangential strains. The differential pressure transducers have a volume measurement resolution of about $1 \mathrm{~mm}^{3}$ and thus leads to a volumetric strain resolution of $10^{-4}$.

\subsubsection{Data acquisition system of the CU HCT device}

Three National Instruments data acquisition and control interface cards (PCI-6052E, PCI6601, and PCI-6703) installed in the personal computer are connected to the multi-channel signal conditioner (MSC-10), the Analog-to-Digital (A/D) converter and the stepper motor controller.

Five stepper motor drivers of the HCT device are connected to the stepper motor controller and driven through the National Instruments PCI-6601 card. Two of five stepper motors which are connected to torque wheel and synchronized to operate on one channel of PCI-6601. Other three steeper motors are connected to the three DPVCs which controls vertical load, inner volume and 
specimen volume during strain controlled loading. Careful selection of this hardware of the HCT device ensures low noise level, and accurate measurement and application of tractions.

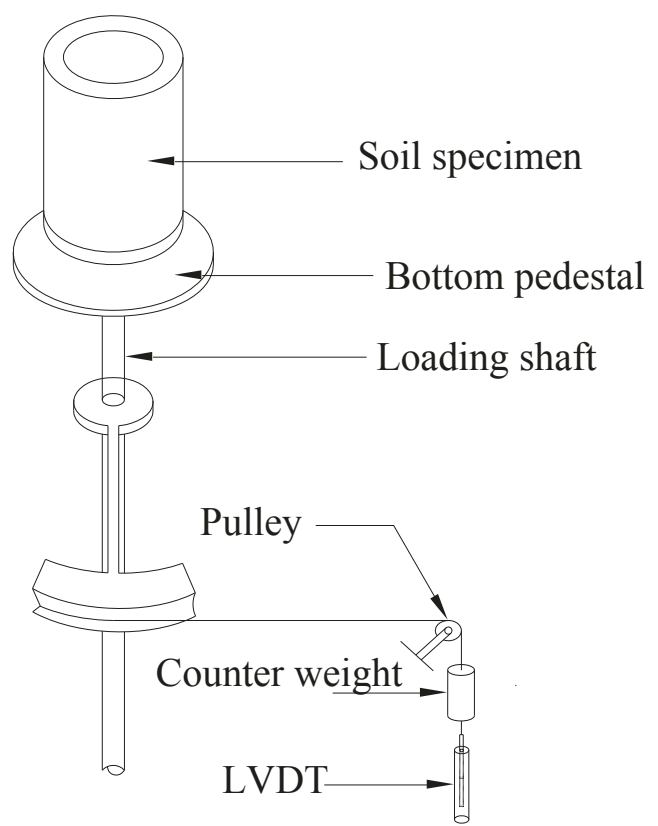

Fig. 3.10 Torsional displacement measurement in the HCT device

The MSC-10 provides excitation, amplification and filtration for the different transducers used, and consists of six analog outputs in which three of them are directly attached to the three EPTs in the HCT device in order to control them. All transducers are read concurrently and analog voltage is collected for a certain period and an average reading from the collected readings during that time interval is determined by an analog integrator. Then, the averaged value is converted to digital output by $\mathrm{A} / \mathrm{D}$ converters.

A in-house developed multithreaded data acquisition program was used to acquire the data and control the system. Multiple execution threads within a single process enable continuous and smooth operation of hardware and proper sampling of input channels without interruption or delay (Logeswaran, 2010). 


\section{EXPERIMENTAL WORK}

\subsection{INTRODUCTION}

This chapter presents details of the experimental work carried out in order to achieve the objectives described in chapter 1. A comprehensive research program was undertaken using the Hollow Cylinder Torsional Shear (HCT) apparatus in order to access the cyclic undrained behaviour of reconstituted Fraser sand specimens. Details of the material used for the testing, specimen reconstitution technique, specimen assembly, and performance and control of the HCT device are also described.

\subsection{MATERiAL TESTED}

The semi-angular Fraser River sand brought from the Fraser Delta in British Columbia was used for this study. Initially the sand was allowed to pass through a set of sieves ranging from $0.850 \mathrm{~mm}$ (\#20 sieve) to $0.075 \mathrm{~mm}$ (\#200 sieve) during a dry sieving process to remove the fine particles which are less than $0.075 \mathrm{~mm}$. Almost all particles were smaller than $0.85 \mathrm{~mm}(100 \%$ passing \#20 sieve). Then the sand retained on the \#200 sieve was wet sieved through \#200 sieve to remove the particles which are less than $0.075 \mathrm{~mm}$. The removal of the particles was undertaken to avoid particle segregation during pluviation and ensure the preparation of uniform specimens. The amount of fine particles less than $0.075 \mathrm{~mm}$ (\#200 sieve) is about $2 \%$ in the original sand, so that the removal of fine particles is expected to not significantly alter the soil response. Fig. 4.1 shows the particles size distribution of the sand as received, and as tested.

The maximum and minimum void ratios of Fraser River sand were determined using ASTM D4253 (2001a) and D4254 (2001b) specifications. The results show that the loosest void ratio $\mathrm{e}_{\max }=0.866$ and the densest void ratio $\mathrm{e}_{\min }=0.511$. According to the ASTM standards for 
test material the average particle size $D_{50}=0.28 \mathrm{~mm}$, uniformity coefficient $C_{u}=2.92$, coefficient of curvature $C_{c}=1.27$ and specific gravity was determined as per ASTM D 854 and found to be $\mathrm{G}_{\mathrm{s}}=2.67$.

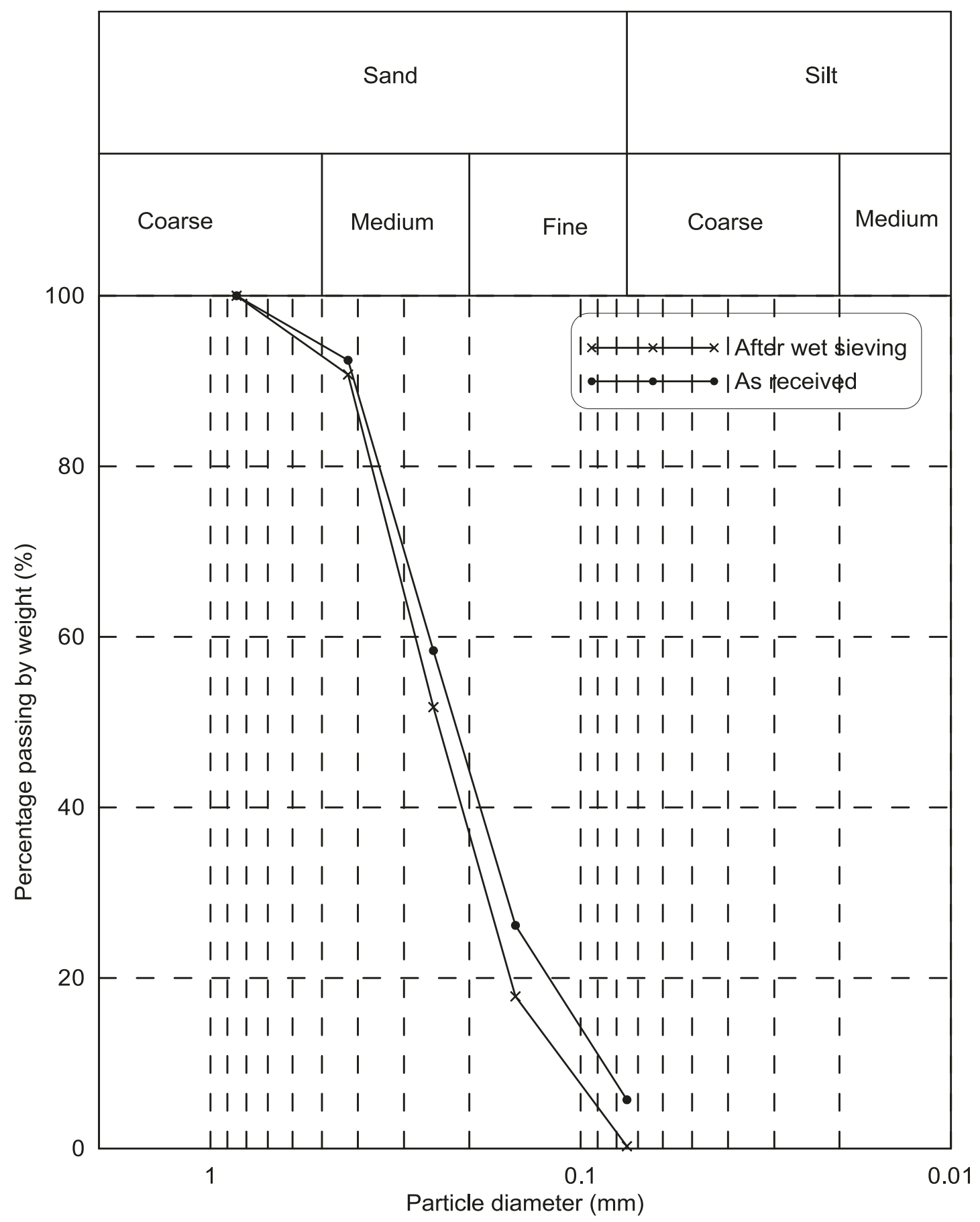

Fig. 4.1 Grain size distribution of Fraser river sand used 


\subsection{SPECIMEN PREPARATION}

In order to duplicate the actual in-situ behaviour as closely as possible during laboratory testing, all tests should preferably be conducted on undisturbed specimens. Undisturbed specimens from sandy soil deposits are however extremely difficult to obtain and conventional soil sampling techniques alter the physical properties of the soil very much. In-situ ground freezing is the best way to obtain undisturbed sandy soil specimens with minimum disturbance and without any alterations on the soil fabric. Because of the high cost associated with this technique, and the natural variability in-situ it is not possible to obtain several undisturbed specimens with identical characteristic for a systematic study. Thus, reconstituted specimens are generally used to research the behaviour of sands in the laboratory.

Many specimen reconstitution techniques have been used in laboratory testing over the years. The common techniques used for specimen reconstitution are moist tamping and air or water pluviation. The ability of the method to produce homogeneous and repeatable specimen of the studied material is a fundamental requirement, and in most cases the adopted reconstitution method is chosen to meet this requirement. Clean sand specimens pluviated in air and water have been shown to yield similar behaviour (Finn et al. 1977), but obtaining fully saturated specimens by air pluviation is a difficult and time consuming task. The most tamping technique compacts the sand at lower water content and may form a honeycomb like structure (Casagrande, 1975) in the specimen which has been shown to be highly collapsible (Vaid et al 2001).

Since water pluviation leads to uniform and repeatable specimens, this technique was used for this experimental study. The specimen reconstituted using this technique closely duplicates the behaviour of fluvial and water deposited soils. Further, undrained shearing requires fully saturated specimen in order to properly access the excess pore pressure generation during 
shearing, and water pluviated sands are excellent in this regard. Density of the water pluviated specimen is not generally affected by the height of the particle drop (Vaid and Negussey, 1988) because particle deposition occurs at constant terminal velocity that is reached within short drop height. However this technique may result in particle segregation if the soil is well graded, but since the sand used for this experimental study is poorly graded and the percentage of fine particles is negligible this technique is deemed most suitable for this experimental study.

\subsubsection{Preliminary steps of specimen preparation}

Preparation of a hollow cylindrical specimen is a complex process, and various steps have to be followed precisely to reconstitute repeatable specimens. Generally, the preliminary steps are completed on the first day, and the actual test is conducted the following day.

A known weight (approximately 5000 g) of Fraser River sand was boiled for about two hours in four volumetric flasks to remove the entrapped air between soil particles. Thereafter the flasks which contain the boiled sand were filled with de-aired water and allowed cool under room temperature. Afterwards, the flasks were sealed by a top cap in order to prevent air contact with de-aired water/sand. All porous stones that are embedded into the end platens were also boiled and allowed to cool under room temperature and kept in a closed container to prevent significant air contact. Adequate amount (about 15 litres) of de- aired water was prepared by boiling the water and allowed to cool to room temperature and seal the top opening in order to prevent the air contact. Both inner and outer membranes were checked to ensure they were not punctured. The O-rings used in the system were checked in order to ensure they were not damaged. Cell, inner, pore pressure reservoirs were filled with de-aired water. 


\subsubsection{Specimen preparation steps}

The base pedestal and the chamber base were cleaned in order to ensure there were no sand particles from the previous tests. This helps to prevent the membrane from puncturing and ensure proper O-ring sealing and avoid water leakage. All drainage and pressure lines were flushed with de-aired water in order to get a fully saturated system. Appropriate reference offsets reading were recorded in the data acquisition program. A dial gauge was fixed to a movable stand and the reference sample height reading was taken on a dummy-sample block to enable accurate determination of initial sample height. The inner membrane was placed around the inner surface of the base platen and held in position using two O- rings. The base platen was fixed to the base pedestal using two locating pins and six evenly spaced screws. At this stage, care was taken to ensure that the pore pressure drainage line in both base pedestal and base platen were coincident with each other. The four piece spilt inner mould was assembled carefully without damaging the inner membrane in order to create the inner cavity and the space between inner membrane and the base platen was filled with water. The inner former was held together with the membrane on the outside, at the bottom by a thick internal metallic ring that snugly fits to the axial shaft, and at the top by an annular platen and by an O-ring. De-aired water was flushed through the cavities in the base platen to saturate the base platen and porous stone cavities. Six porous stones were transferred into the de-aired water filled cavities carefully from a de-aired water filled container without any significant air contact. Again de-aired water was flushed through the porous stone and allowed to flood the base platen. This way the air contact was prevented from any part of the devices which is in contact with the soil specimen. 
The outer membrane was placed in position and sealed with the outer surface of the base platen using two O-rings. The two piece outer mould was placed in position carefully without damaging the outer membrane and held together using two metal rings at top and bottom respectively. The outer membrane was flipped over the mould at the top and sealed with the outer surface of the outer mould using two O-rings. A small amount of suction applied through the opening in the outer mould to keep the membrane attached to the interior wall of the outer mould. The inner surface of the outer mould is lined with porous plastic, and pulls the outer membrane taut under the applied suction. This creates a smooth membrane lined annular cavity, which is filled with de-aired water. Any air bubbles in the water filled annular cavity were removed manually as much as possible in order to ensure getting a fully saturated specimen. An extension collar was placed at the top of the outer mould and filled with water up to the possible level without spilling the water into the inner cavity. The flasks which contain boiled sand were filled with de-aired water and inverted into the cavity formed by the two membranes which was filled with de-aired water. The sand was deposited into the annular cavity under gravity by mutual displacement of water and sand. The submerged tip of the flask was slowly circulated along the cavity in order to facilitate the sand pluviation with approximately level surface. The uniform level of sand ensures the horizontal plane of deposition. Since the final velocity of the falling sand particles is reached with in short depth the drop height does not affect the density very much (Vaid and Negussey, 1988). The deposition was continued until the required level was reached and the excess sand was removed by siphoning off and a level top surface was established. The siphoned sand was oven dried and weighted to determine the weight of sand used which use in the calculation of density or void ratio of the specimen. Fig $4.2 \& 4.3$ show 
the details of the specimen deposition method and a schematic illustration of the specimen set up respectively.

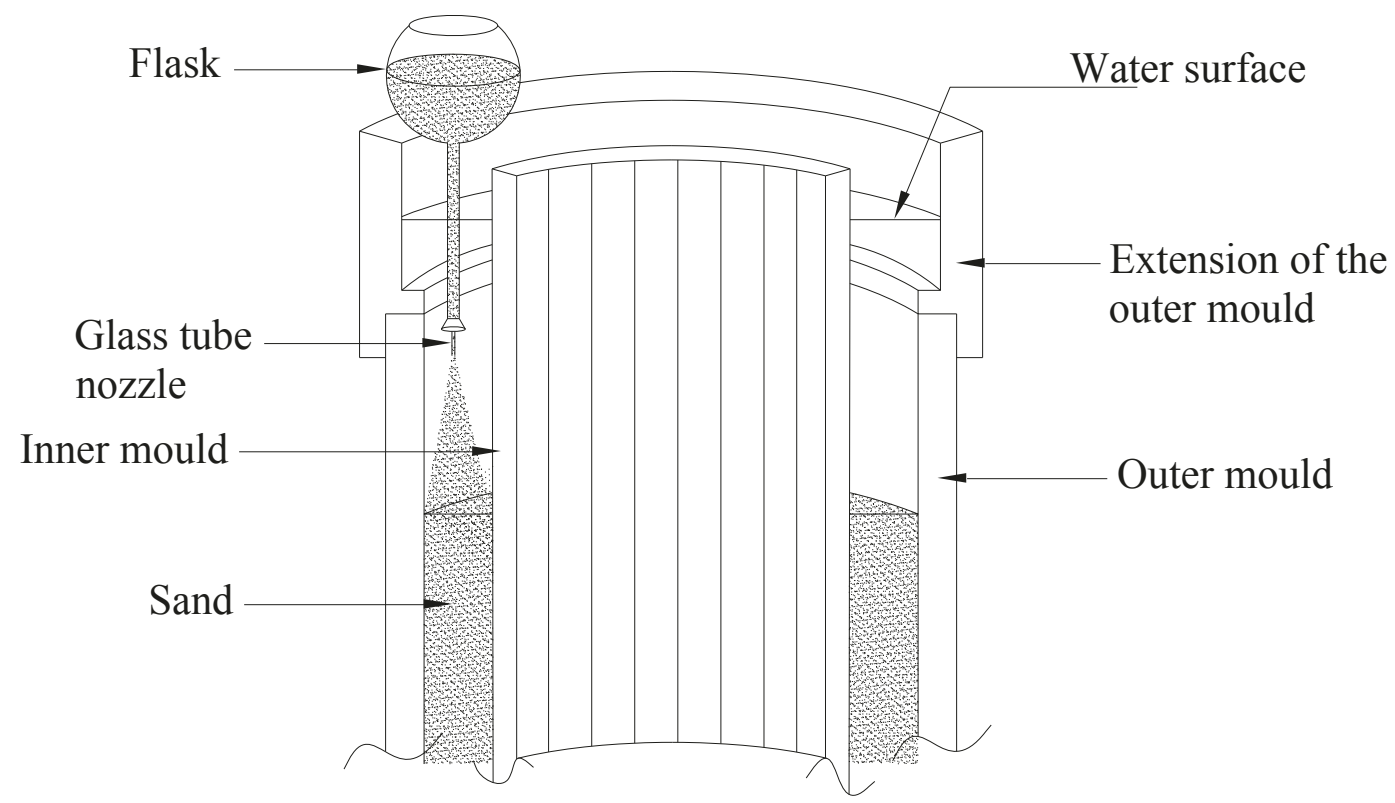

Fig. 4.2 HCT specimen preparation by water pluviation technique (After Sayao 1989)

The saturated bottom half of top platen with saturated porous stones was carefully seated on top of the sand specimen without disturbing the specimen. The dial gauge was set in place to provide the initial reading, and continuous monitoring of the height changes from this stage forward. De-aired water from the pore water reservoir was carefully circulated upwards from the bottom (with a very low gradient - typically about 0.1 or less) in order to remove the air entrapped between the top outer vertical wall of the platen and the outer membrane. The outer membrane was flipped on to the top platen and sealed with two O-rings. Again de-aired water was allowed to percolate from the bottom to remove the air entrapped between interior wall of the top platen and the inner membrane. 


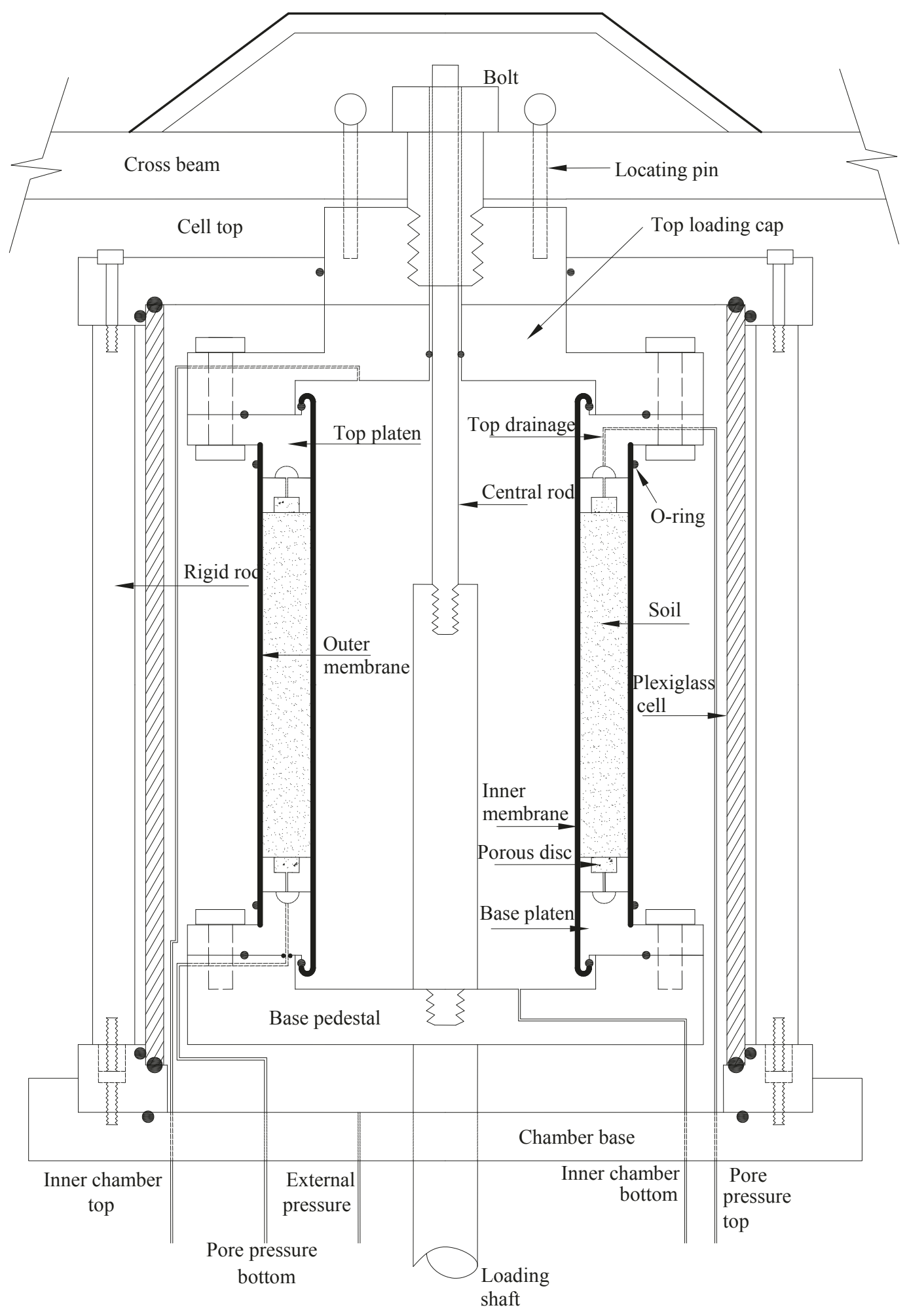

Fig. 4.3 Details of HCT specimen setup (After Logeswaran 2010) 
The inner membrane was flipped on to the top platen and sealed with two O-rings. The two screws and drainage ports of the top platens were opened, and de-aired water was allowed to percolate again slowly upwards through the specimen with small gradient to remove entrapped air bubbles between the top platen and the specimen. The top drainage line was connected to the drainage port of the top platen. Then approximately $20 \mathrm{kPa}$ of suction was applied to the specimen and the volume of water drained from the specimen was measured. Continuous volume measurement was carried out from this stage until the end of the test. With the applied suction, the soil will have an effective stress and can stand on its own even after the inner and outer moulds are removed. The inner former was carefully removed without disturbing the specimen nor damaging the inner membrane. The inner cavity was filled with de-aired water up to the top and air bubbles were removed manually as much as possible. The top half of the top platen was placed and tightened with the bottom half of the top platen with six evenly placed screws and two locating pins. The outer former vacuum was removed and consequently the outer former also removed. The full saturation of the inner cavity was ensured and the drainage line for the inner top was connected to the platen.

The O-ring used to seal the cell chamber was placed in the slot on the chamber base. The cell chamber was placed in position and tight with chamber base with six screws and filled with water. After that the cell top was placed on top of the cell chamber and sealed with the chamber by six screws. The top cross beam was brought into the position and fixed with the reaction frame. The central rod which is used to measure vertical displacement was fixed with the loading shaft and its seals the inner chamber. The specimen was moved up until the top cap contacts the cross beam by pressuring the bottom chamber of vertical loading double acting piston. The top cap was attached with cross beam by a bolt in order to ensure vertical alignment of the axes of 
the specimen and the frame. The two locating pins protruding from the cross beam were inserted into loading cap in order to prevent rotational movement during the application of torque loading. The specimen height, inner diameter and outer diameter were calculated at this stage from the total height change and the volume change which were tracked up to this point. The calculated specimen height, inner diameter and outer diameter were inputted into the data acquisition system and all stresses, and deformations are monitored continuously through the data acquisition program from this stage forward.

The specimen was made undrained and $30 \mathrm{kPa}$ of confining pressure was applied to the specimen hydrostatically to bring the pore pressure to a positive value before starting the saturation check. Then saturation check phase of the test program was conducted and the Skempton's $B$ value was checked in several increments of confining pressure. Full saturation was confirmed for the specimens (a Skempton's $B$ value of 0.99 or higher was considered indicative of full saturation). Specimens which had a lower level of saturation were rectified using the back pressure saturation technique, and all tests were conducted on specimens with Skempton's $B$ value of 0.99 or higher.

After completing the saturation check phase the specimen was consolidated to the desired initial stress state using the fully automated feedback control loading system. The desired initial stress state, number of increments and the loading rate were inputted into the data acquisition system prior to start the consolidation phase. All specimens for this test program were consolidated for an initial effective mean normal stress state of $200 \mathrm{kPa}$. This effective stress was reached in $25 \mathrm{kPa}$ stress increments with a five minute delay in between successive loading stages. Moreover the $25 \mathrm{kPa}$ stress increment was applied in ten steps during a three minute interval to ensure that the prescribed loading path is closely followed using feedback control. 
Following the consolidation phase the shearing phase of the testing program was executed with the predetermined test parameters. All cyclic tests were carried out with a cyclic period of 240 seconds in order to run the feedback control loading system properly. The loading rate has no effect of the cyclic undrained behaviour of sands, and thus the slower loading rate was not a concern.

\subsection{VOID RATIO CALCULATION}

Calculation of the void ratio of the specimen is important in laboratory soil testing in order to compare a series of tests to enable appropriate comparisons. The specific gravity of the soil, mass of the dry soil used for the sample preparation and the total volume of the specimen are required to calculate the void ratio. Usually the volume of the specimen is calculated from the height and the diameter of the specimen which is measured using the conventional methods. The height of the specimen can be measured confidently compared to the measurement of diameter.

For HCT specimens the diameter is measured according to the suggestion made by Vaid and Sivathayalan (1996). The outer diameter of the specimen is determined from the height of the water in the membrane lined cavity formed by the outer mould when it filled by water. The outer diameter of the specimen is calculated using the mass of water needed to fill the cavity, the density of the water at the room temperature and the height of the water column in the membrane lined cavity. In order to determine the inner diameter the cavity formed by the inner and outer membrane is filled with water and the height of water column in that cavity is measured. Similar to outer diameter the inner diameter also calculated using the mass of water needed to fill the cavity, the density of the water at the room temperature and the height of the water column in the membrane lined cavity. Since the volume and the height changes of the specimen was continually monitored during the sample preparation the void ratio of the specimen was 
calculated accurately. Given the care taken in calculating the void ratios, the void ratios reported in this thesis are accurate to \pm 0.005 . 


\section{INFLUENCE OF PRINCIPAL STRESS DIRECTIONS ON LIQUEFACTION RESISTANCE}

\subsection{INTRODUCTION}

The results from the tests which were carried out in order to study the effect of initial principal stress direction $\left(\alpha_{\sigma c}\right)$ and level of rotation $\left(\Delta \alpha_{\sigma}\right)$ on the liquefaction resistance of sands are presented in this chapter. The tests were carried out using the hollow cylinder torsional shear (HCT) apparatus described in chapter 3. As noted earlier, commonly used test methods to assess the liquefaction potential of soils in laboratory (i.e. triaxial and simple shear tests) cannot properly simulate the stress conditions in-situ. During a cyclic triaxial test the major principal stress may be either oriented along the vertical deposition direction or along the horizontal bedding plane and undergo a sudden jump rotation. During a simple shear test principal stress will undergo smooth rotation in uncontrolled manner. However in the field the principal stress may be oriented along an arbitrary direction and undergo smooth rotation. Investigation on cyclic soil behaviour under an arbitrary initial principal stress direction and possible levels of rotation may be useful to improve the current knowledge about liquefaction assessment.

Fig 5.1 shows the nature of the rotation of principal stress directions imposed on Fraser River sand for this experimental study program. The direction of principal stress was rotated smoothly during the application of cyclic stress. The direction of principal stress was rotated in a sinusoidal form with the frequency equal to the frequency of the applied cyclic shear stresses. In other words, the occurrence of the largest inclination of principal stress corresponds to the occurrence of the maximum cyclic shear stress. Cyclic deviator stress was considered to be positive for one half, and negative for the other half of the loading cycle. The sign of the 
direction of deviatoric stress are defined based on the direction of torsional shear stress $\tau_{z \theta}$. The positive values of the deviatoric stress denote that $\tau_{z \theta}$ is counter clockwise and the negative values denote that $\tau_{z \theta}$ is clockwise.
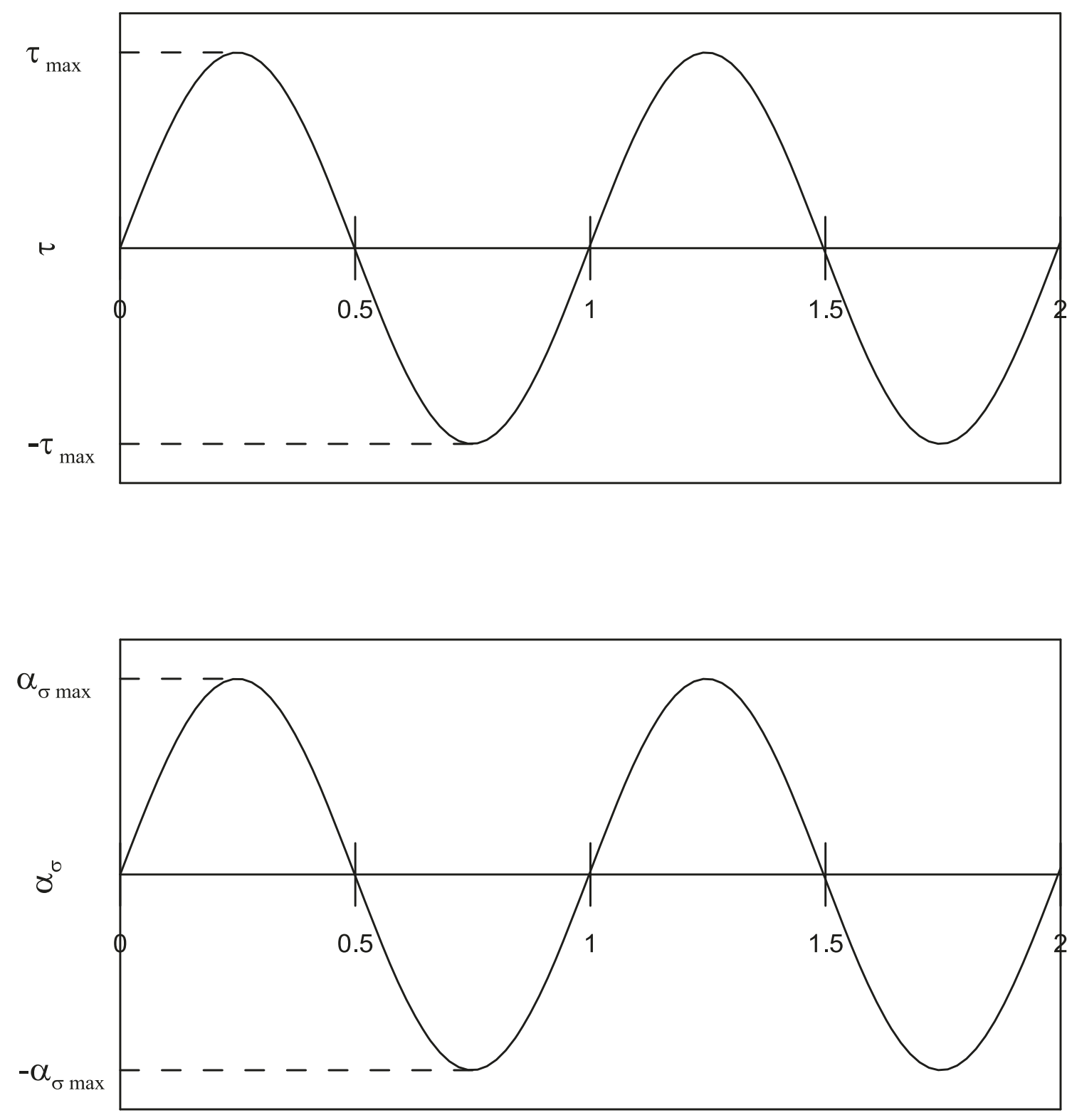

$\mathrm{N}$

Fig 5.1: Cyclic shear stress and direction of principal stresses 
The intensity of the cyclic loading (called the cyclic stress ratio, CSR) has been expressed by normalizing the cyclic shear stress $\left(\sigma_{d, c y} / 2\right)$ by the effective mean normal stress in the hollow cylinder test as follows (Logeswaran, 2010)

$$
\frac{\sigma_{\mathrm{d}}}{2 \sigma_{\mathrm{mc}}^{\prime}}=\beta_{0}+\beta_{\mathrm{max}} \sin (\omega \mathrm{t})
$$

Here, $\sigma_{d}$ is the deviatoric stress, $\sigma_{m c}^{\prime}$ is effective mean normal stress at the end of consolidation, $\beta_{0}$ is the initial static shear stress ratio and $\beta_{\max }$ is the amplitude of the cyclic stress ratio. $\beta_{0}$ is is zero in the series of tests reported herein because all specimens were consolidated isotropically.

The direction of the major principal stress $\alpha_{\sigma}$ was changed in a sinusoidal manner in order to smoothly rotate the principal stress direction during the application of cyclic shear stress as noted in the following equation

$$
\alpha_{\sigma}=\alpha_{\sigma c}+\alpha_{\sigma_{\max }} \sin (\omega \mathrm{t})
$$

Here, $\alpha_{\sigma}$ is principal stress direction with specimen deposition axis, $\alpha_{\sigma c}$ is initial principal stress direction at the end of consolidation. $\alpha_{\sigma_{\max }}$ is allowed maximum principal stress rotation.

The torsional shear stress applied on the horizontal plan $\tau_{z \theta}$ can be expressed in terms of $\sigma_{d}$ and $\alpha_{\sigma}$ using Mohr's circle.

$$
\tau_{\mathrm{z} \theta}=\frac{\sigma_{\mathrm{d}} \sin 2 \alpha_{\sigma}}{2}
$$

From the above equations during the smooth rotation of principal stress the shear stress on the horizontal plan can be calculated as follows (Logeswaran, 2010)

$$
\tau_{\mathrm{z} \theta}=\left(\beta_{0}+\beta_{\max } \sin (\omega \mathrm{t})\right) * \sin 2 \alpha_{\sigma} * \sigma_{\mathrm{mc}}^{\prime}
$$




\subsection{TEST PROGRAM}

The undrained behaviour of sand under the applied monotonic, cyclic loading is studied in terms of developed excess pore pressure, developed maximum shear strain and the number of cycles required to trigger liquefaction. The feedback controlled data acquisition system was used throughout the test in order to reach the targeted stress state.

All sand specimens were reconstituted using water pluviation and were consolidated to a hydrostatic effective mean normal stress $\left(\sigma_{m c}^{\prime}\right)$ of $200 \mathrm{kPa}$. The effective stress ratio at the end of consolidation is thus $k_{c}=1$. Technically, the values of $b_{\sigma c}$ and $\alpha_{\sigma c}$ are undefined at this stage, but this initial state permits cyclic shear with principal stress rotation about initial principal stress directions $\left(\alpha_{\sigma c}\right)$ of $0^{\circ}, 30^{\circ} 45^{\circ}, 60^{\circ}$ and $90^{\circ}$ under approximately plane strain conditions (intermediate stress parameter $b_{\sigma c}=0.4$ ). The relative density at the end of the consolidation was $33.5 \pm 3 \%$. All cyclic tests were conducted with a cyclic stress ratio (CSR) of 0.2 but with different level of stress rotations $\left(\Delta \alpha_{\sigma}=0^{\circ}, 30^{\circ}, 45^{\circ}, 60^{\circ}\right.$ and $\left.90^{\circ}\right)$. During the cyclic loading total mean normal stress $\sigma_{m}$ was held constant to ensure that the measured response reflects the shear induced behaviour. The period of cyclic loading was maintained at 240 seconds to enable proper feedback control. Liquefaction was defined according to the maximum shear strain criterion and the specimen was deemed to have liquefied when the maximum shear strain $\gamma_{\max }$ exceeded $3.75 \%$ (NRC 1985). The details of the stress rotation tests conducted are given in Table 5.1 and

\section{2 .}


Table 5.1 Initial and loading states of tests conducted under Monotonic loading

\begin{tabular}{|c|c|c|c|c|c|c|}
\hline \multirow{2}{*}{ Test No } & \multicolumn{4}{|c|}{ Consolidation state } & \multicolumn{2}{c|}{ Loading state } \\
\cline { 2 - 7 } & $D_{r c}(\%)$ & $\sigma_{m c}^{\prime}(\mathrm{kPa})$ & $b_{c}$ & $\alpha_{\sigma c}(\mathrm{deg})$ & $\Delta \alpha_{\sigma}(\mathrm{deg})$ & $\Delta \sigma_{d} / \Delta \alpha_{\sigma}$ \\
\hline MO01 & 36.5 & 200 & 0.4 & 45 & +30 & 13.33 \\
\hline MO02 & 36.0 & 200 & 0.4 & 45 & +45 & 8.89 \\
\hline MO03 & 34.9 & 200 & 0.4 & 45 & +60 & 6.67 \\
\hline MO04 & 35.5 & 200 & 0.4 & 45 & +90 & 4.44 \\
\hline MO05 & 35.8 & 200 & 0.4 & 0 & +45 & 8.89 \\
\hline MO06 & 35.9 & 200 & 0.4 & 90 & +45 & 8.89 \\
\hline
\end{tabular}

\subsection{MONOTONIC RESPONSE UNDER PRINCIPAL STRESS ROTATION}

A limited number of monotonic tests were conducted to understand the influence of initial principal stress direction and its subsequent rotation on the monotonic undrained behaviour of Fraser River sand. Three specimens starting at $\alpha_{\sigma c}=0^{\circ}, 45^{\circ}$ and $90^{\circ}$ were subjected to monotonic shearing with a fixed rate of principal stress rotation. Prior to shearing, the stress states of each specimen was $\sigma_{m c}^{\prime}=200 \mathrm{kPa}$ and $D_{r c}=33.5 \%$. Shearing was carried out by holding total mean normal stress $\sigma_{m}$ and the intermediate principal stress parameter $\left(b_{\sigma}\right)$ constant as shown in Table 5.1. During shearing $\Delta \sigma_{d} / \Delta \alpha_{\sigma}$ was held constant (at $8.89 \mathrm{kPa} /$ degree which is equivalent to $\Delta \alpha=45^{\circ}$ over a $200 \mathrm{kPa}$ shear stress increment ${ }^{1}$ ). This loading represents simultaneous increase in $\sigma_{d}$ and $\alpha_{\sigma}$ that would be expected in several in-situ loading situations.

\footnotetext{
${ }^{1}$ All $\Delta \alpha$ values reported in this section are similar equivalent rotation angles normalized to $200 \mathrm{kPa}$ shear stress, and the actual magnitude of the stress rotation would be smaller if the maximum shear stress did not reach $200 \mathrm{kPa}$.
} 
Table 5.2 Initial and loading states of tests conducted under cyclic loading

\begin{tabular}{|c|c|c|c|c|c|c|c|}
\hline \multirow{2}{*}{ Test No } & \multicolumn{4}{|c|}{ Consolidation state } & \multicolumn{2}{|c|}{ Loading state } & \multirow{2}{*}{$\begin{array}{c}\text { Number } \\
\text { of } \\
\text { Cycles }\end{array}$} \\
\hline & $D_{r c}(\%)$ & $\sigma_{m c}^{\prime}(\mathrm{kPa})$ & $b_{c}$ & $\alpha_{\sigma c}(\operatorname{deg})$ & $\alpha_{\sigma}(\operatorname{deg})$ & $\frac{\sigma_{d}}{2 \sigma_{m c}^{\prime}}$ & \\
\hline CY01 & 30.6 & 200 & 0.4 & 0 & \pm 0 & 0.2 & - \\
\hline CY02 & 31.4 & 200 & 0.4 & 0 & \pm 30 & 0.2 & 14 \\
\hline CY03 & 30.5 & 200 & 0.4 & 0 & \pm 45 & 0.2 & 8 \\
\hline CY04 & 36.5 & 200 & 0.4 & 0 & \pm 60 & 0.2 & 10 \\
\hline CY05 & 30.7 & 200 & 0.4 & 0 & \pm 90 & 0.2 & 15 \\
\hline CY06 & 35.6 & 200 & 0.4 & 30 & \pm 0 & 0.2 & - \\
\hline CY07 & 33.5 & 200 & 0.4 & 30 & \pm 30 & 0.2 & 19 \\
\hline CY08 & 30.5 & 200 & 0.4 & 30 & \pm 45 & 0.2 & 7 \\
\hline CY09 & 36.5 & 200 & 0.4 & 30 & \pm 60 & 0.2 & 12 \\
\hline CY10 & 36.5 & 200 & 0.4 & 30 & ${ }_{-9}^{+90}$ & 0.2 & 18 \\
\hline CY11 & 35.3 & 200 & 0 & 45 & \pm 0 & 0.2 & - \\
\hline CY12 & 35.2 & 200 & 0 & 45 & \pm 30 & 0.2 & 18 \\
\hline CY13 & 31.2 & 200 & 0 & 45 & \pm 45 & 0.2 & 16 \\
\hline CY14 & 31.4 & 200 & 0 & 45 & \pm 60 & 0.2 & 19 \\
\hline CY15 & 30.5 & 200 & 0 & 45 & ${ }_{-9}^{+90}$ & 0.2 & 20 \\
\hline CY16 & 36.5 & 200 & 0.4 & 60 & ${ }_{-}^{ \pm} 0$ & 0.2 & - \\
\hline CY17 & 31.4 & 200 & 0.4 & 60 & ${ }_{-30}^{+30}$ & 0.2 & 12 \\
\hline CY18 & 31.2 & 200 & 0.4 & 60 & \pm 45 & 0.2 & 9 \\
\hline CY19 & 36.5 & 200 & 0.4 & 60 & \pm 60 & 0.2 & 12 \\
\hline CY20 & 33.7 & 200 & 0.4 & 60 & ${ }_{-90}^{+90}$ & 0.2 & 18 \\
\hline CY21 & 36.1 & 200 & 0.4 & 90 & \pm 0 & 0.2 & - \\
\hline CY22 & 36.5 & 200 & 0.4 & 90 & \pm 30 & 0.2 & 9 \\
\hline CY23 & 35.5 & 200 & 0.4 & 90 & \pm 45 & 0.2 & 7 \\
\hline CY24 & 35.3 & 200 & 0.4 & 90 & \pm 60 & 0.2 & 9 \\
\hline CY25 & 36.5 & 200 & 0.4 & 90 & ${ }_{-90}^{+90}$ & 0.2 & 27 \\
\hline
\end{tabular}


Figure 5.2 presents the effective stress paths and deviator stress strain response of Fraser River sand sheared at a fixed rotation rate of $\Delta \sigma_{d} / \Delta \alpha_{\sigma}=8.89$ but at different initial $\alpha_{\sigma c}$ values. Significant differences can be noted in the measured response, especially when comparing the response at $\alpha_{\sigma c}=0^{\circ}$ with the other two cases. Fairly profound undrained anisotropy is apparent in these water deposited sand specimens. As the direction of $\sigma_{1}$ during consolidation deviates from the deposition direction $\left(\alpha_{\sigma c}=0^{\circ}\right)$ towards that of the bedding plane $\left(\alpha_{\sigma c}=90^{\circ}\right)$, undrained response changes from dilative to contractive for a fixed rotation. The sand exhibits highest strength at $\alpha_{\sigma c}=0^{\circ}$ and essentially similar response when starting at $\alpha_{\sigma c}=45^{\circ}$ or $90^{\circ}$. Several previous studies (Logeswaran, 2010, Sivathayalan, 2000, Uthayakumar, 1995, Yoshimine et al., 1998) report a systematic reduction in the undrained strength as the direction of the major principal stress aligns toward the bedding planes, and the lowest strength is generally observed when $\alpha_{\sigma c}=90^{\circ}$. Sayao (1989) and Wijewickreme (1990) have observed a similar behaviour in drained response, in which volumetric deformation for a given $\sigma_{d}$ increased with increasing $\alpha_{\sigma}$. All of these previous observations were from the results of tests where there is no principal stress rotation; thus, principal stress is fixed at the position of consolidation direction or in other words $\Delta \alpha_{\sigma}=0^{\circ}$.

Rotation of principal stress causes the inclination of the major principal stress to increase in the $\alpha_{\sigma c}=45^{\circ}$ test, and decrease in the $\alpha_{\sigma c}=90^{\circ}$ test. Thus these two tests exhibit relatively similar behaviour when shearing occurs with principal stress rotation. The rotation of major principal stress direction with maximum shear strain under controlled principal rotation is shown in Fig. 5.2. The observation shows that during $\alpha_{\sigma c}=0^{\circ}$ test principal stress was rotated by $20.1^{\circ}$ whereas during $\alpha_{\sigma c}=45^{\circ}$ and $\alpha_{\sigma c}=90^{\circ}$ tests principal stress was rotated by $16.2^{\circ}$ and $6.5^{\circ}$ respectably at the stage of PT. 

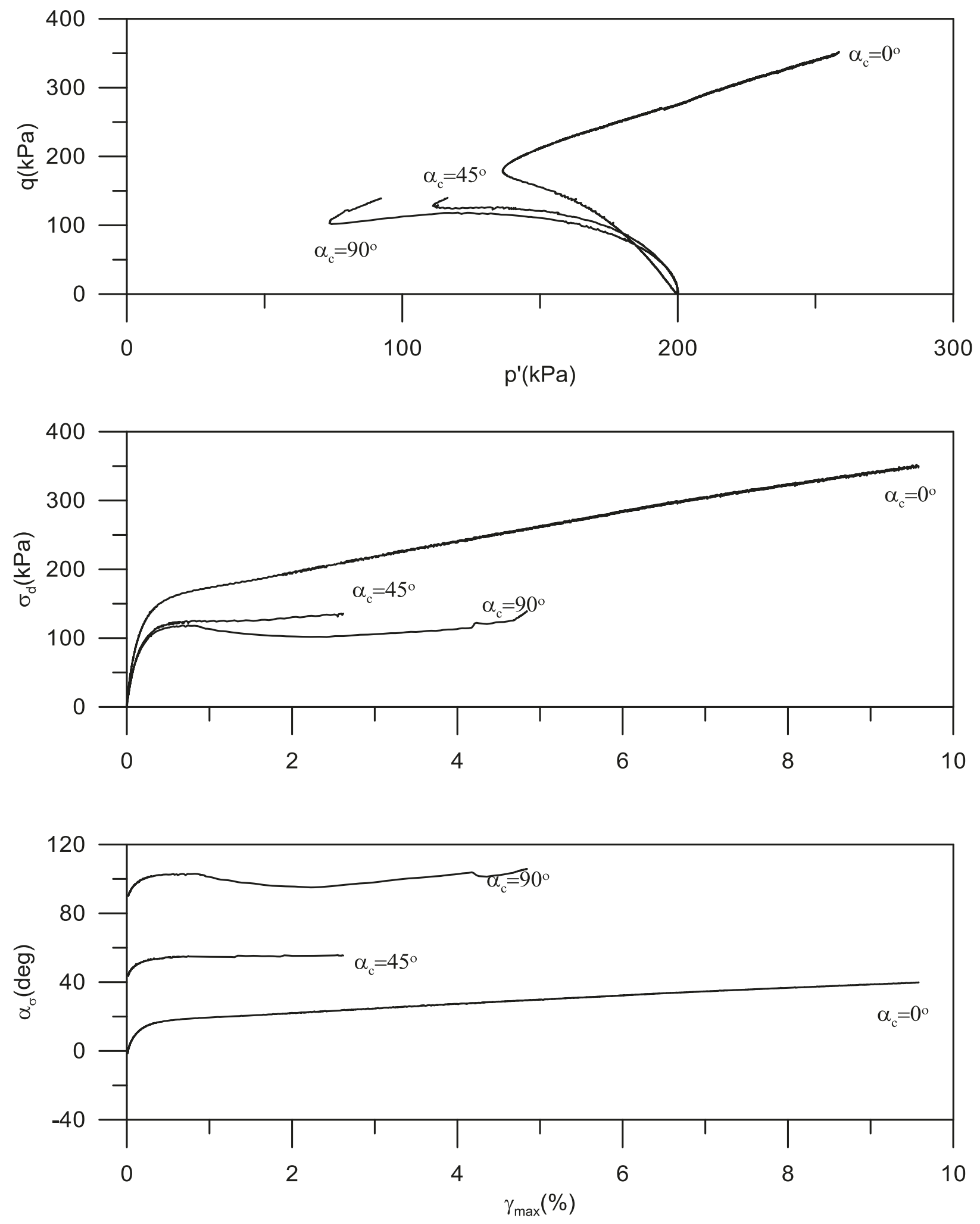

Fig 5.2 Effective stress path, deviator stress and major principal stress direction variation with maximum shear strain 
A series of monotonic undrained tests in which $\alpha_{\sigma}$ was increased in a controlled manner is described in this section. Prior to shearing, all specimens were isotropically consolidated to an effective stress of $200 \mathrm{kPa}$. The targeted relative density was 33.5\%. During shear loading $\sigma_{m}$, $b, \frac{\Delta \sigma_{d}}{\Delta \alpha_{\sigma}}$ were held constant. These tests represents simultaneous increase in $\sigma_{d}$ and $\alpha_{\sigma}$ and evaluate the effect of the degree of stress rotation on the undrained response.

Figure 5.3 shows effective stress path and deviator stress- strain response for tests conducted at a fixed initial $\alpha_{\sigma c}=45^{\circ}$ but different rates of stress rotation $\Delta \alpha_{\sigma}$ values. The degree of contractiveness may be noted to increase with an increase in $\Delta \alpha_{\sigma}$, and the response at the smallest $\Delta \alpha_{\sigma}$ used was only marginally contractive. Clearly, a faster rate of stress rotation leads to a weaker response, and consequently lower undrained strength. The rotation of major principal stress direction with maximum shear strain under controlled principal rotation is shown in Fig 5.3. The result shows $\Delta \alpha_{\sigma}=30^{\circ}$ test undergoes a rotation of $10.3^{\circ}, \Delta \alpha_{\sigma}=45^{\circ}$ test $16.2^{\circ}$, $\Delta \alpha_{\sigma}=60^{\circ}$ test $18.3^{\circ}$ and $\Delta \alpha_{\sigma}=90^{\circ}$ test $27.8^{\circ}$ before reaching the PT line. Since the magnitude of stress rotation was related to the deviatoric stress, principal stress directions varied little in the tests where the material strain softened and deformed at essentially constant shear stress.

Effective stress conditions at phase transformation (PT) state and maximum obliquity (MO) state for both fixed rotation of principal stress $\left(\Delta \alpha_{\sigma}=45^{\circ}\right)$ loading and different rotation of principal stress tests $\left(\alpha_{\sigma c}=45^{\circ}\right)$ are shown in Fig. 5.4. The results show that the friction angle mobilized at PT is equal to $\emptyset_{P T}=36.4^{\circ}$ and the friction angle mobilized at MO is equal to $\emptyset_{M O}=39.3^{\circ}$. Even though there is some scatter in the data, these results agree with the contention that the friction angle mobilized at the phase transformation state is a material property, and independent of whether the principal stress directions remain fixed or vary during shearing. 

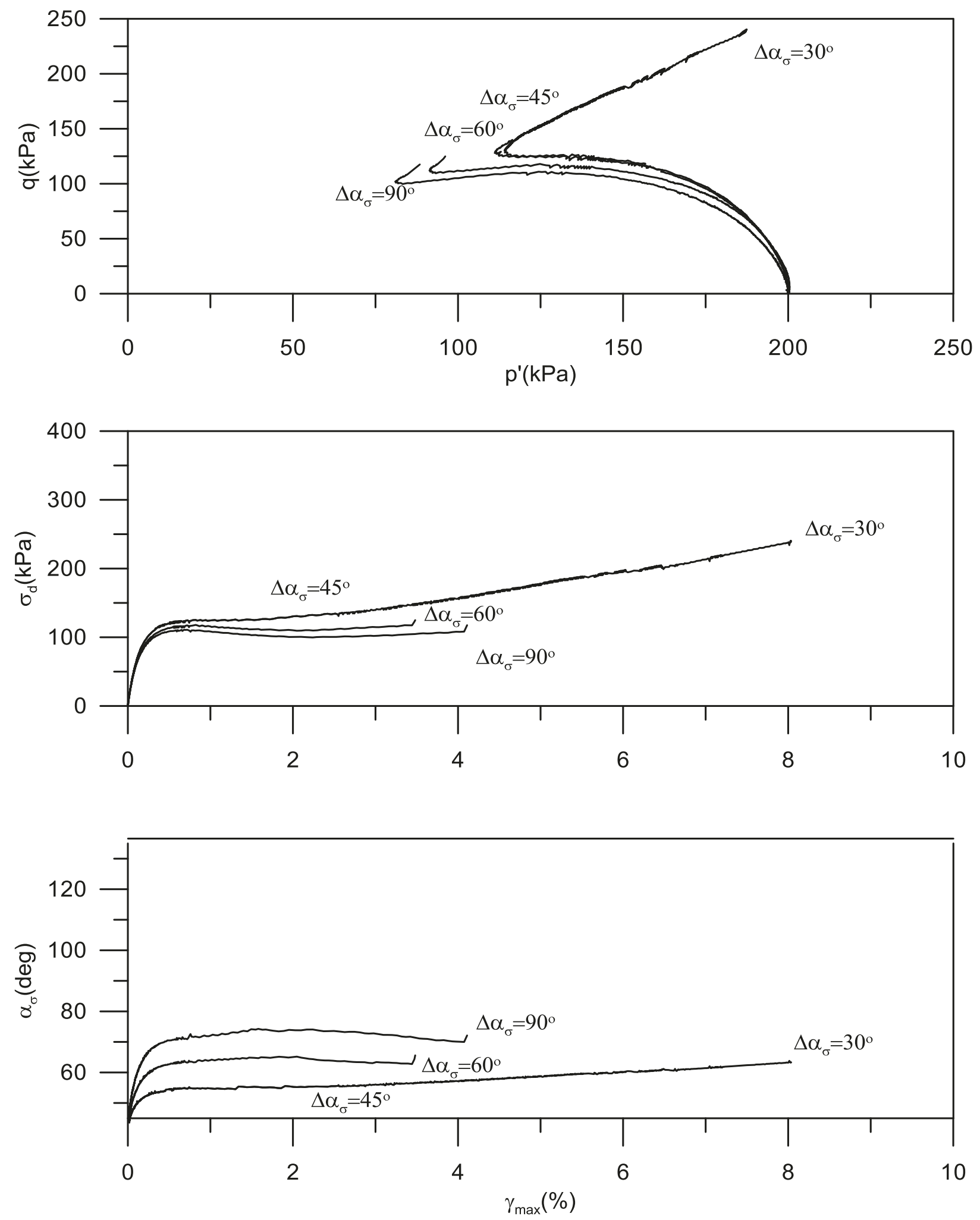

Fig 5.3 Effective stress path, deviator stress and major principal stress direction variation with maximum shear strain 

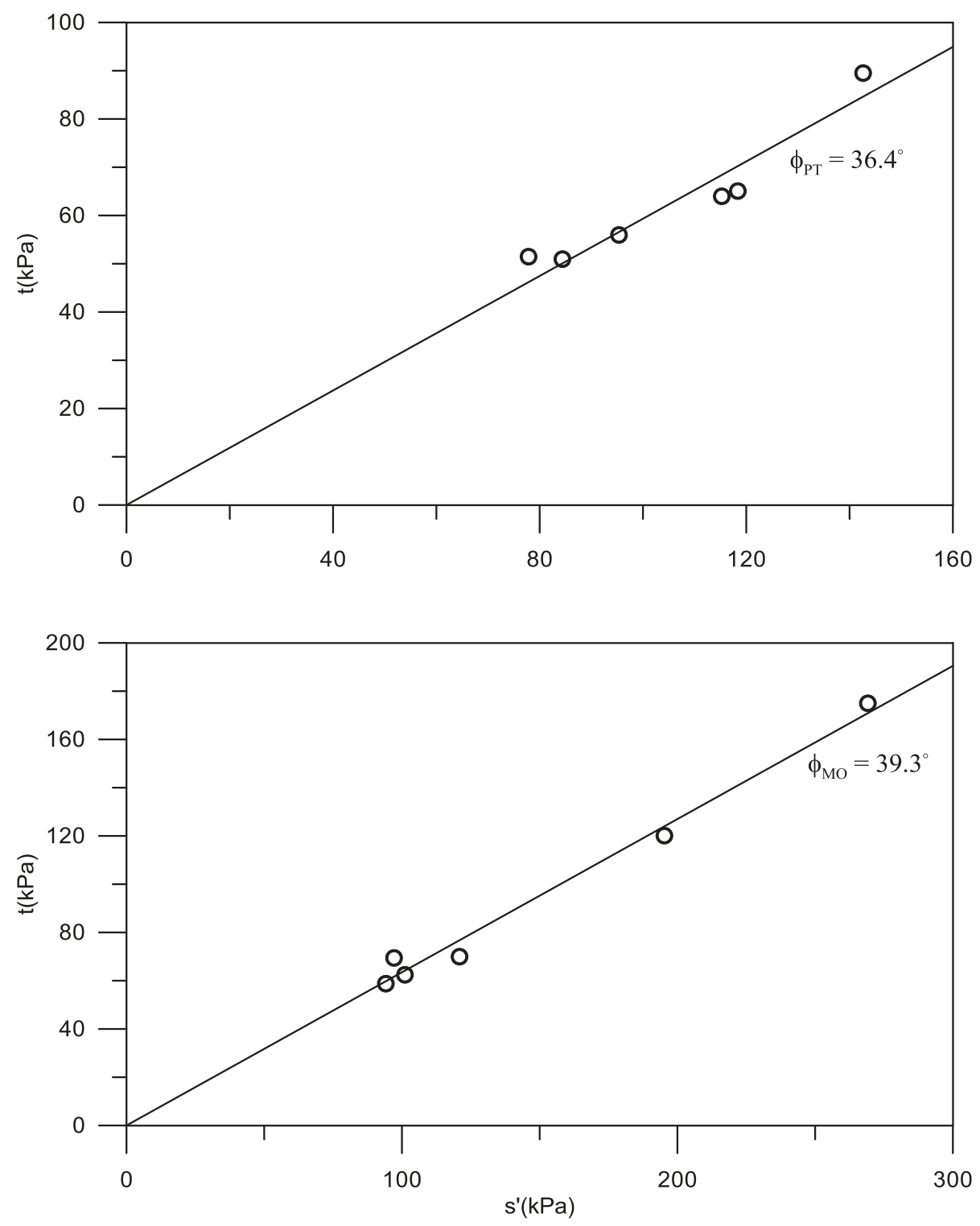

Fig 5.4 Variation of stress state at PT and MO state

Figure 5.5(a) and (b) present the relationship between phase transformation strength and rotation angle $\Delta \alpha_{\sigma}$, and Figure 5.5(c) and (d) show the variation of the phase transformation strength with the inclination of the major principal stress at phase transformation. Figures 5.5(a) and (c) show the data for fixed initial angle $\left(\alpha_{\sigma c}=45^{\circ}\right)$ but different magnitudes of stress rotation, and the other two figures show the data for a fixed rotation of $\left(\Delta \alpha=45^{\circ}\right)$ but starting at different $\alpha_{\sigma c}$ values. The continuous decrease in $S_{P T}$ with increase in $\Delta \alpha_{\sigma}$ starting at a given $\alpha_{\sigma c}$ 
demonstrates that $S_{P T}$ reduces when the principal stress direction moves from the direction of soil deposition to bedding plane. Clearly a plot with the inclination of $\sigma_{1}$ at PT and that with $\Delta \alpha$ are analogous if the initial angle is fixed. On the other hand, when comparing different initial angles $\left(\alpha_{\sigma c}\right)$ the magnitude of stress rotation at PT state and the $S_{P T}$ decrease with the increase in initial angle $\left(\alpha_{\sigma c}\right)$. However the $S_{P T}$ decreases with increase in the inclination of the major principal stress at the instant of phase transformation even for different $\alpha_{\sigma c}$ case.
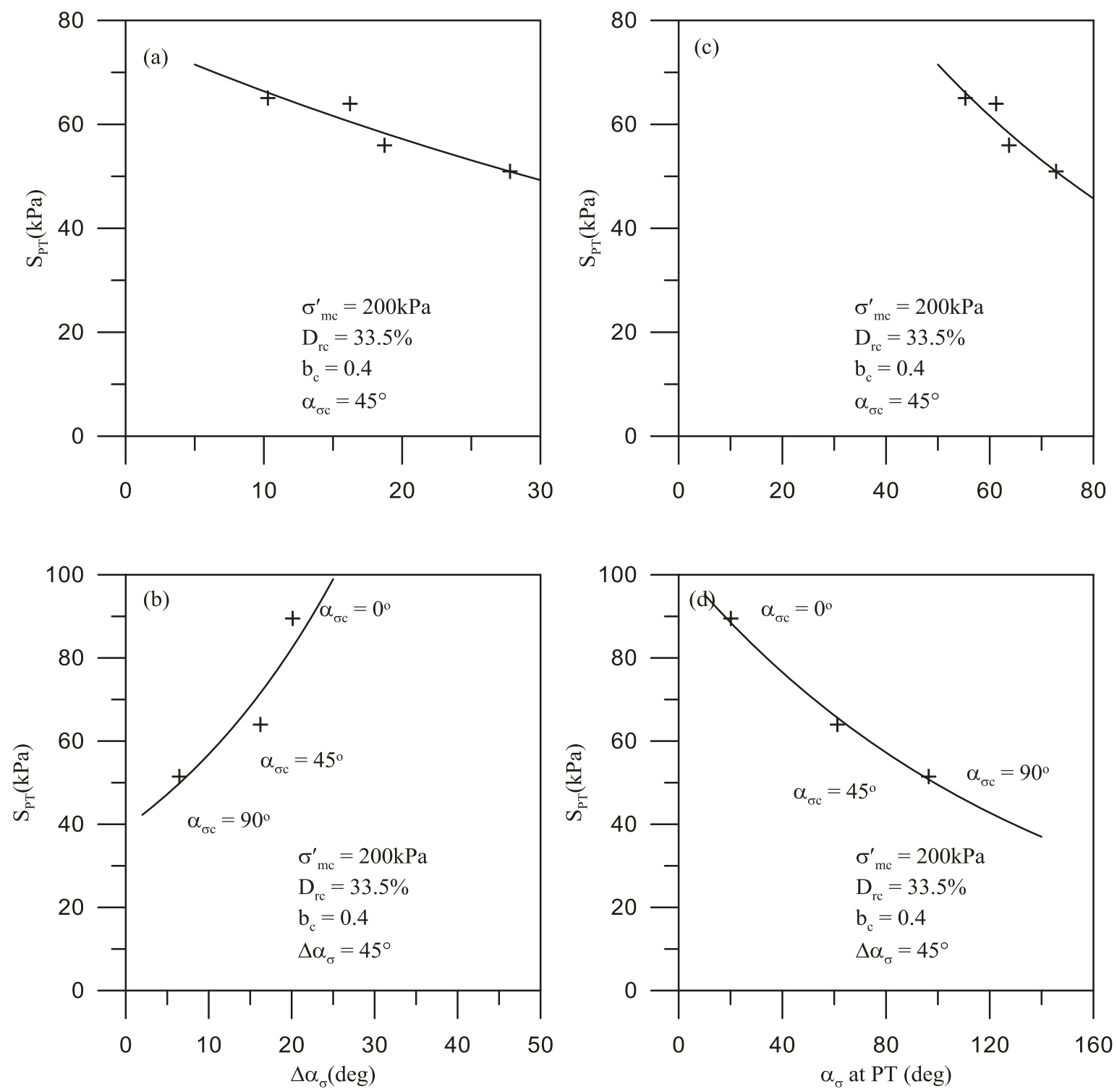

Fig 5.5 Variation of phase transformation strength with $\Delta \alpha_{\sigma}$ and $\alpha_{\sigma}$ at PT state 


\subsection{INFLUENCE OF $\Delta \alpha_{\sigma}$ ON CYCLIC BEHAVIOUR OF SAND}

A series of cyclic tests were performed at a fixed cyclic stress ratio (CSR) of $\frac{\sigma_{d}}{2 \sigma^{\prime} m c}=0.2$ with different amounts of smooth principal stress rotation. Principal stress directions were rotated in a smooth manner about the vertical deposition axis of the specimen (axis of cross anisotropy), and about axes inclined at $30^{\circ}, 45^{\circ}, 60^{\circ}$ and $90^{\circ}$ to the vertical. During these tests, the total mean normal stress was held constant at $\sigma_{m c}=450 \mathrm{kPa}$ and the intermediate principal stress parameter $b$ was held constant at $b_{c}=0.4$ for most of the tests except for the tests with the initial principal stress direction of $45^{\circ}$ with vertical. Test results of specimens subjected to different magnitudes of stress rotation about a specific principal stress axes direction are presented in the following sections for each principal stress axes inclination.

\subsubsection{Behaviour for $\alpha_{\sigma c}=0^{\circ}$}

The first series of tests were conducted by applying a cyclic shear stress symmetrical about the vertical axis. The applied cyclic stress ratio of $\mathrm{CSR}=0.2$ requires a maximum cyclic shear stress amplitude of $40 \mathrm{kPa}$, but depending on the degree of stress rotation different levels of cyclic torsional shear stress $\tau_{z \theta}$ would be required to follow the loading path. Fig. 5.6 shows the variation of $\tau_{z \theta}$ during different levels of the smooth rotation of the principal stress direction required to produce a sinusoidal cyclic shear stress. For this particular case $\left(\alpha_{\sigma c}=0^{\circ}\right)$ when maximum rotation of principal stress direction $\alpha_{\sigma \max }$ is $45^{\circ}$ or less $\left(30^{\circ}\right) \tau_{z \theta}$ reaches the peak at the instant of the peak cyclic stress ratio and when $\alpha_{\sigma m a x}$ is greater than $45^{\circ}\left(60^{\circ}\right.$ and $\left.90^{\circ}\right) \tau_{z \theta}$ does not reaches the peak at the instant of the peak cyclic stress ratio. Further, no torsional shear stress on the horizontal plane is required $\left(\tau_{z \theta}=0\right)$ for the case where there is no rotation of principal stress, and thus the major principal stress remained along the vertical deposition 
direction whereas for $\Delta \alpha_{\sigma}=90^{\circ}$ test $\tau_{z \theta_{\max }}$ of $22.5 \mathrm{kPa}$ is applied. Even though $\Delta \alpha_{\sigma}=30^{\circ}$ and $60^{\circ}$ tests show almost equal $\tau_{z \theta_{\max }}$ of $34.6 \mathrm{kPa}$ and $34.7 \mathrm{kPa}$ respectively, the variation within cycle is different. For $\Delta \alpha_{\sigma}=45^{\circ}$, since the major principal stress is oriented $45^{\circ}$ apart from horizontal at the instance of maximum rotation of principal stress, the $\tau_{z \theta_{\max }}$ which is equal to 40 $\mathrm{kPa}$ is mobilized.

Fig. 5.7 and 5.8 show the variation of deviatoric stress, excess pore pressure and maximum shear strain with number of cycles for $\Delta \alpha_{\sigma}=0^{\circ}$ and $\Delta \alpha_{\sigma}=60^{\circ}$ respectively. The deviatoric stress varies in a cyclic manner with the maximum deviatoric stress of $80 \mathrm{kPa}$ throughout the loading in the $\Delta \alpha_{\sigma}=0^{\circ}$ test and during the first eight cycles in $\Delta \alpha_{\sigma}=60^{\circ}$ test. Prescribed stress paths cannot be followed during strain softening deformation, and this is noted during cycles 9 and 10 in the $\Delta \alpha_{\sigma}=60^{\circ}$ test. The plot shows the variation of deviatoric stress (which is technically always positive) with the sign of $\tau_{z \theta}$ to illustrate the cyclic variation of the shear stress. There is no $\tau_{z \theta}$ for $\Delta \alpha_{\sigma}=0^{\circ}$ test, and the shear stress is always applied in the same direction on the $45^{\circ}$ plane. Thus Fig. 5.7 shows only positive variation of deviatoric stress. On the other hand for $\Delta \alpha_{\sigma}$ $=60^{\circ}$ test deviatoric stress shows both negative and positive variation (according to the sign of $\left.\tau_{z \theta}\right)$ indicating that the shear stress reverses directions during the loading. Even though there are peaks and troughs in pore pressure response within a cycle the cumulative excess pore pressure increases with the number of cycles in both cases. Almost $100 \%$ excess pore pressure is mobilized in the $\Delta \alpha_{\sigma}=60^{\circ}$ when the specimen liquefied during the $10^{\text {th }}$ cycle, but excess pore pressure and shear strain development were fairly small in the $\Delta \alpha_{\sigma}=0^{\circ}$ test. Since the increment in the excess pore water pressure decreases the strength of the soil, the maximum shear strain $\left(\gamma_{\max }\right)$ shows a systematic accumulation with number of cycle as noted in the excess pore pressure generation. Induced $\gamma_{\max }$ is relatively low until the specimen reaches the PT state and 
the rate of strain development rapidly increases afterwards. As mentioned above since $\Delta \alpha_{\sigma}=60^{\circ}$ test liquefied during the $10^{\text {th }}$ cycle the $\gamma_{\max }$ reaches the limiting value of $4 \%$ at the time of liquefaction and there is no liquefaction for $\Delta \alpha_{\sigma}=0^{\circ}$ test.

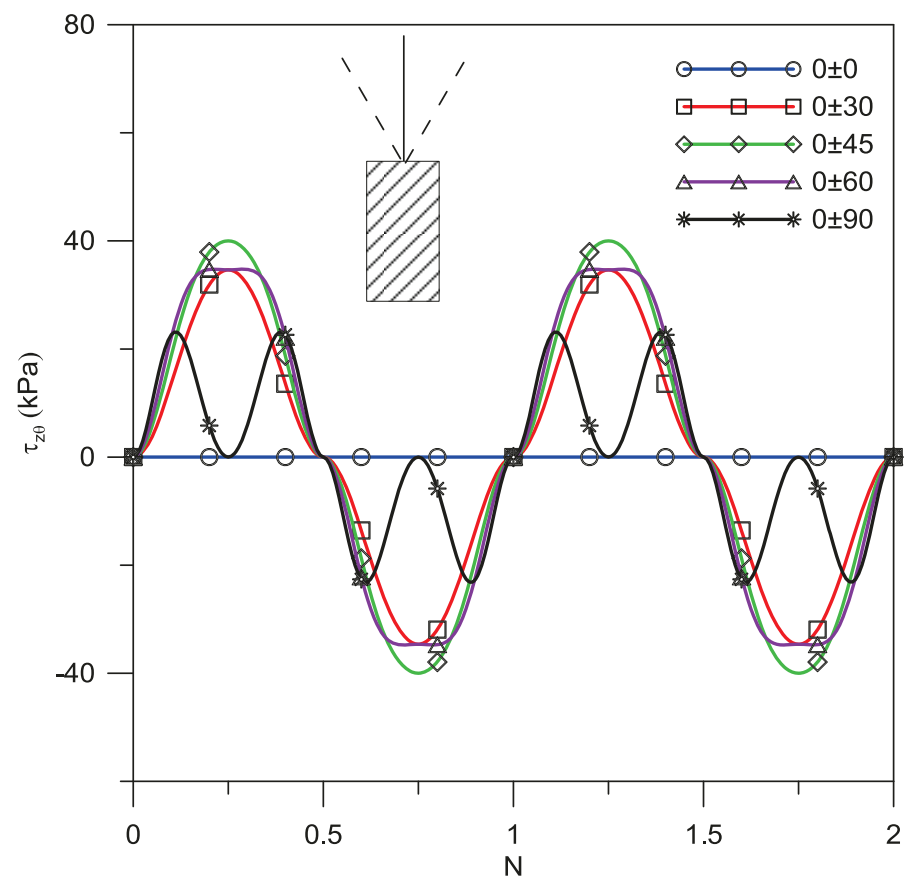

Fig 5.6 Shear stress on the horizontal plane during smooth rotation of $\alpha_{\sigma}$ from $\alpha_{\sigma c}=0^{\circ}$ 

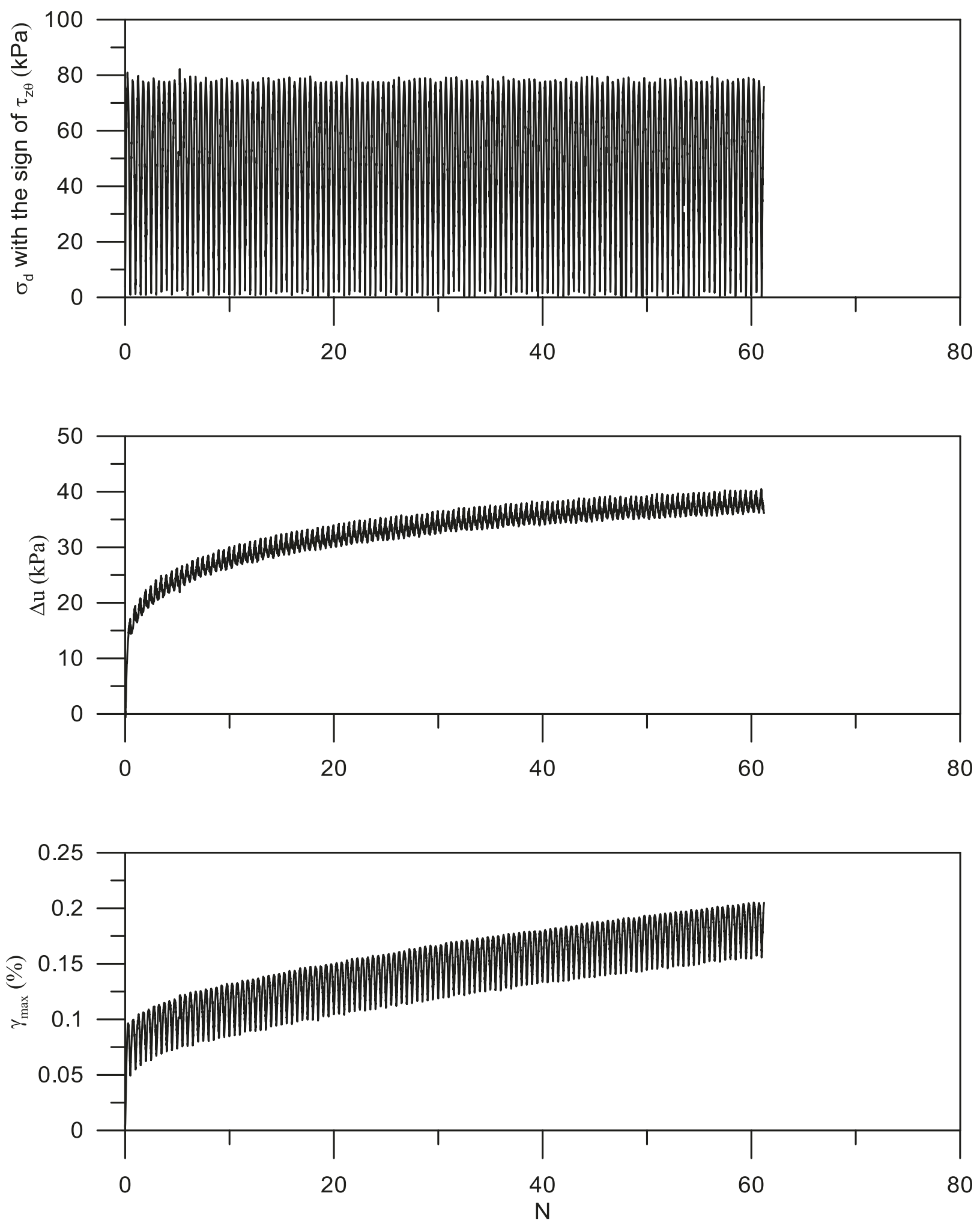

Fig. 5.7 Variation of deviatoric stress, excess pore pressure and maximum shear strain with number of cycles for $\Delta \alpha_{\sigma}=0^{\circ}$ 

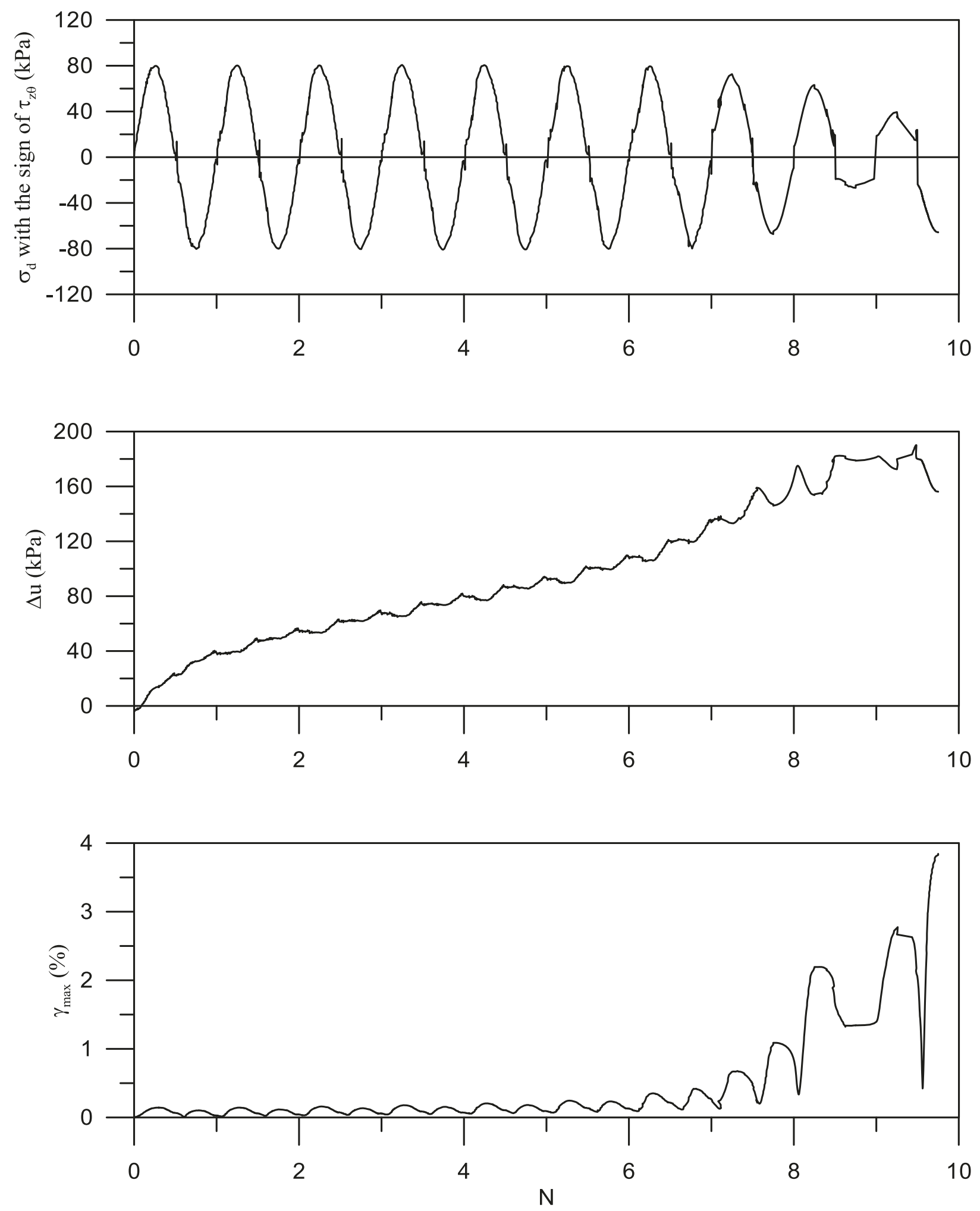

Fig. 5.8 Variation of deviatoric stress, excess pore pressure and maximum shear strain with number of cycles for $\Delta \alpha_{\sigma}=60^{\circ}$ 
Fig. 5.9 and 5.10 show the variation of total vertical stress, effective vertical stress and torsional shear stress applied on the horizontal plane with number of cycles for the $\Delta \alpha_{\sigma}=0^{\circ}$ and $\Delta \alpha_{\sigma}=60^{\circ}$ tests respectively. Since all the tests are carried out at constant mean normal stress $\left(\sigma_{m}\right)$ the total vertical stress varies in a cyclic manner between $450 \mathrm{kPa}$ and $490 \mathrm{kPa}$ during the $\Delta \alpha_{\sigma}=0^{\circ}$ test and between $430 \mathrm{kPa}$ to $460 \mathrm{kPa}$ during $\Delta \alpha_{\sigma}=60^{\circ}$ test. On the other hand effective vertical stress decreases with the number of cycle as the excess pore pressure goes up and eventually reaches a state of zero defined as liquefaction for the $\Delta \alpha_{\sigma}=60^{\circ}$ test. For the $\Delta \alpha_{\sigma}=0^{\circ}$ test major principal stress remains along the vertical deposition direction, and thus there is no torsional shear stress applied on the horizontal plane $\left(\tau_{z \theta}=0\right)$ whereas for the $\Delta \alpha_{\sigma}=60^{\circ}$ test the torsional shear stress applied on the horizontal plane $\left(\tau_{z \theta}=0\right)$ varies in a cyclic manner with a maximum magnitude of $34.7 \mathrm{kPa}$.

Fig. 5.11 and 5.12 show the variation of vertical strain, radial strain and torsional shear strain applied on the horizontal plane with number of cycles for the $\Delta \alpha_{\sigma}=0^{\circ}$ and $\Delta \alpha_{\sigma}=60^{\circ}$ tests respectively. Strain development is small in the $\Delta \alpha_{\sigma}=0^{\circ}$ test as the loading did not generate significant excess pore water pressures. For the $\Delta \alpha_{\sigma}=60^{\circ}$ test, relatively low strains were induced until the specimen reached the PT state, and there is a rapid increase in both vertical and torsional shear strains during the liquefaction cycle. Since all these tests were performed under plane strain condition $\left(b_{c}=0.4\right)$ there should be no strain along the radial direction since the intermediate principal strain must be zero. However, plane strain conditions cannot be enforced during strain softening deformation and thus some radial strain occurs during the load cycles leading to liquefaction. For the $\Delta \alpha_{\sigma}=0^{\circ}$ test since there is no $\tau_{z \theta_{\max }}$ as noted in Fig. 5.9 there is no torsional shear strain on the horizontal plan whereas for the $\Delta \alpha_{\sigma}=0^{\circ}$ test there is low shear 
strain varies in a cyclic manner until the specimen reaches PT state and large shear strain is developed after that.
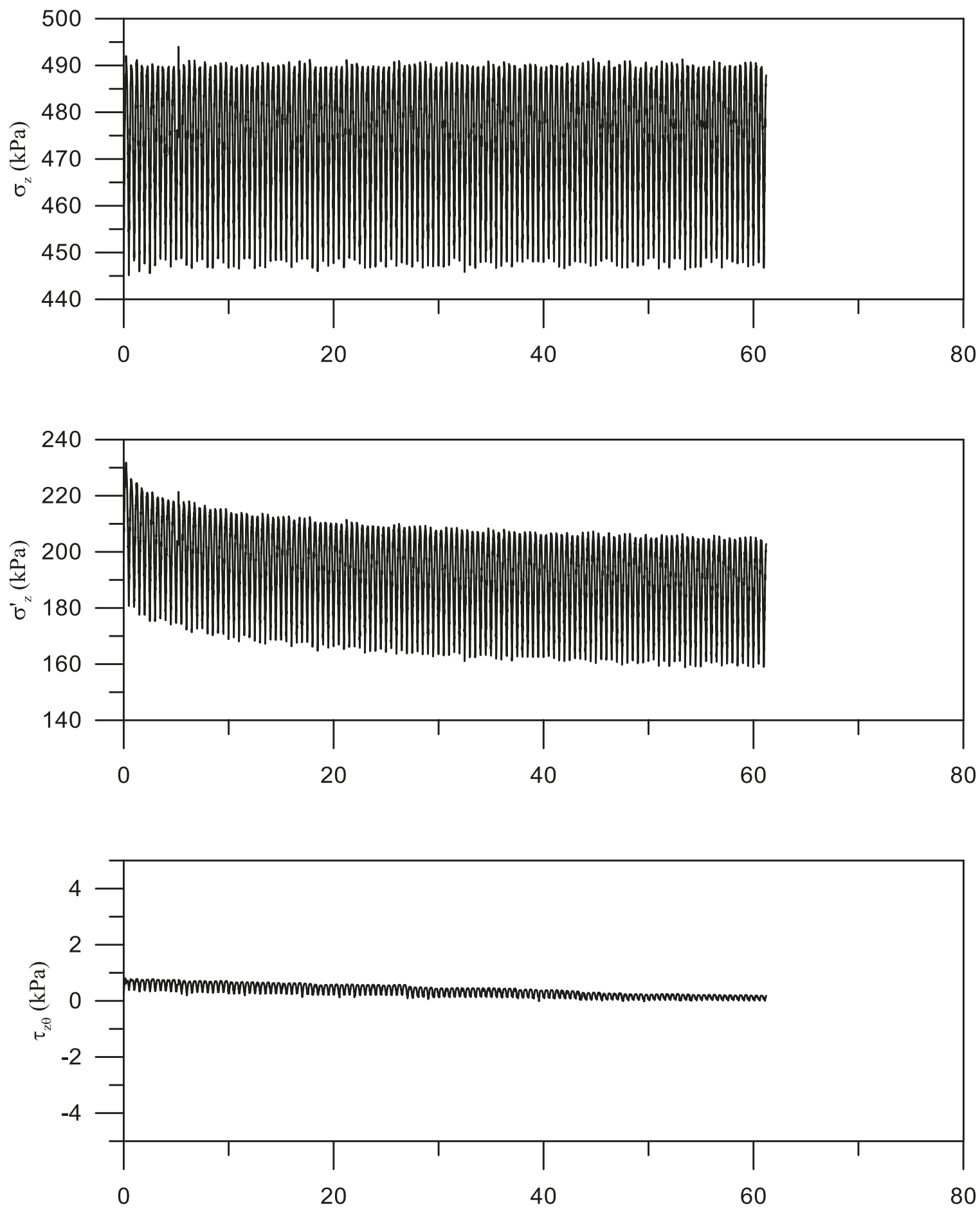

Fig. 5.9 Variation of total vertical stress, effective vertical stress and torsional shear stress applied on the horizontal plane with number of cycles for $\Delta \alpha_{\sigma}=0^{\circ}$ 

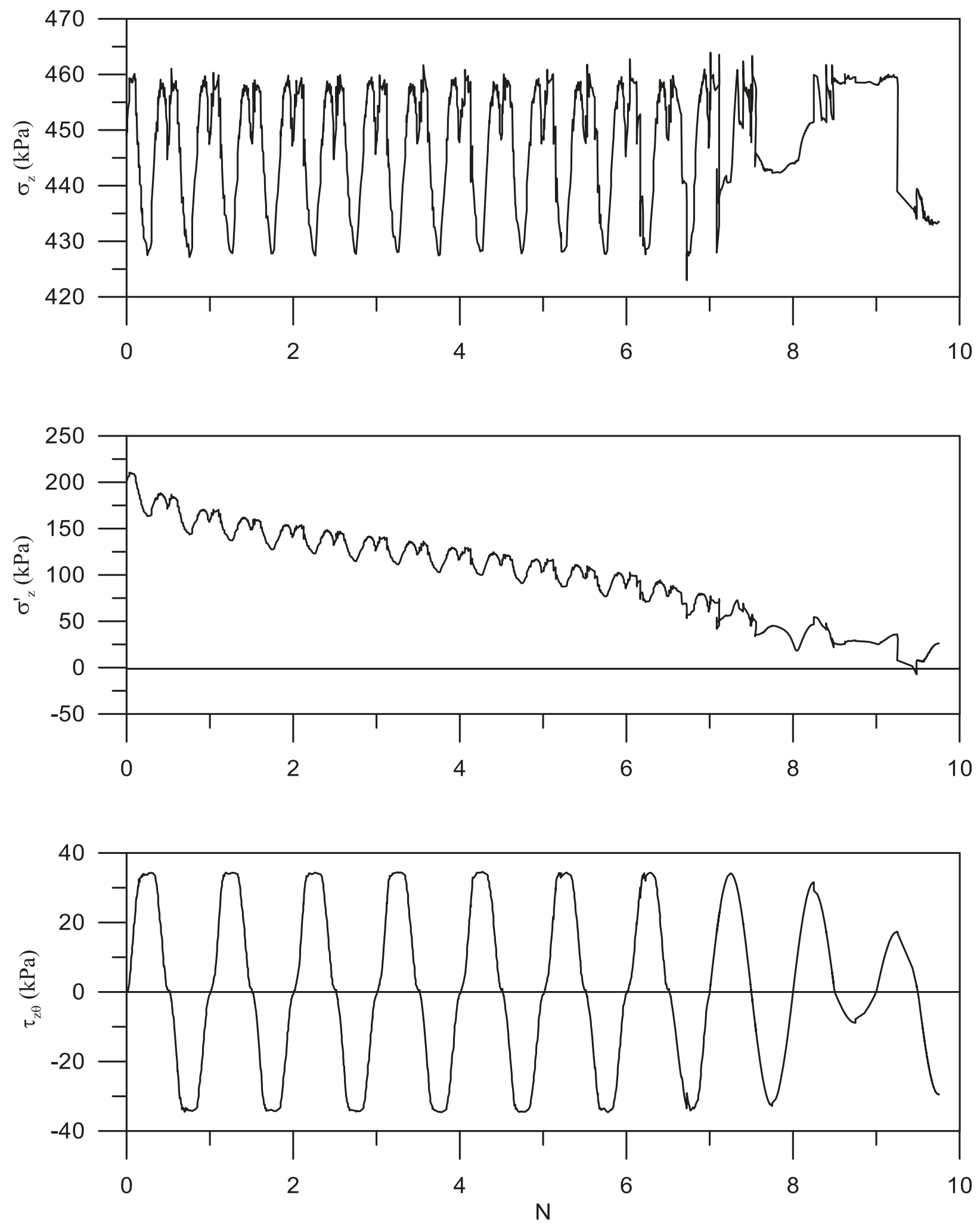

Fig. 5.10 Variation of total vertical stress, effective vertical stress and torsional shear stress applied on the horizontal plane with number of cycles for $\Delta \alpha_{\sigma}=60^{\circ}$ 

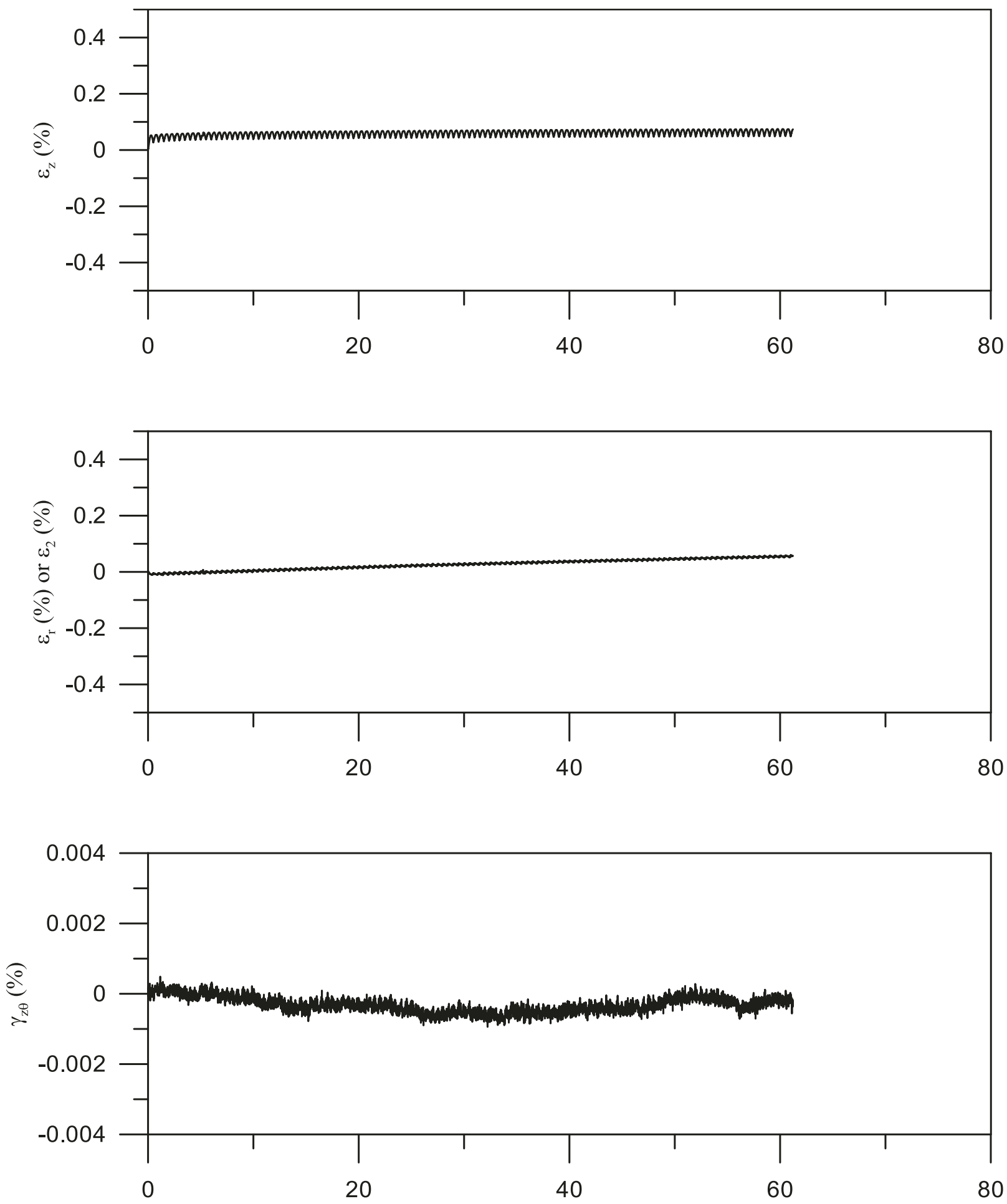

Fig. 5.11 Variation of vertical strain, radial strain and torsional shear strain applied on the horizontal plane with number of cycles for $\Delta \alpha_{\sigma}=0^{\circ}$ 

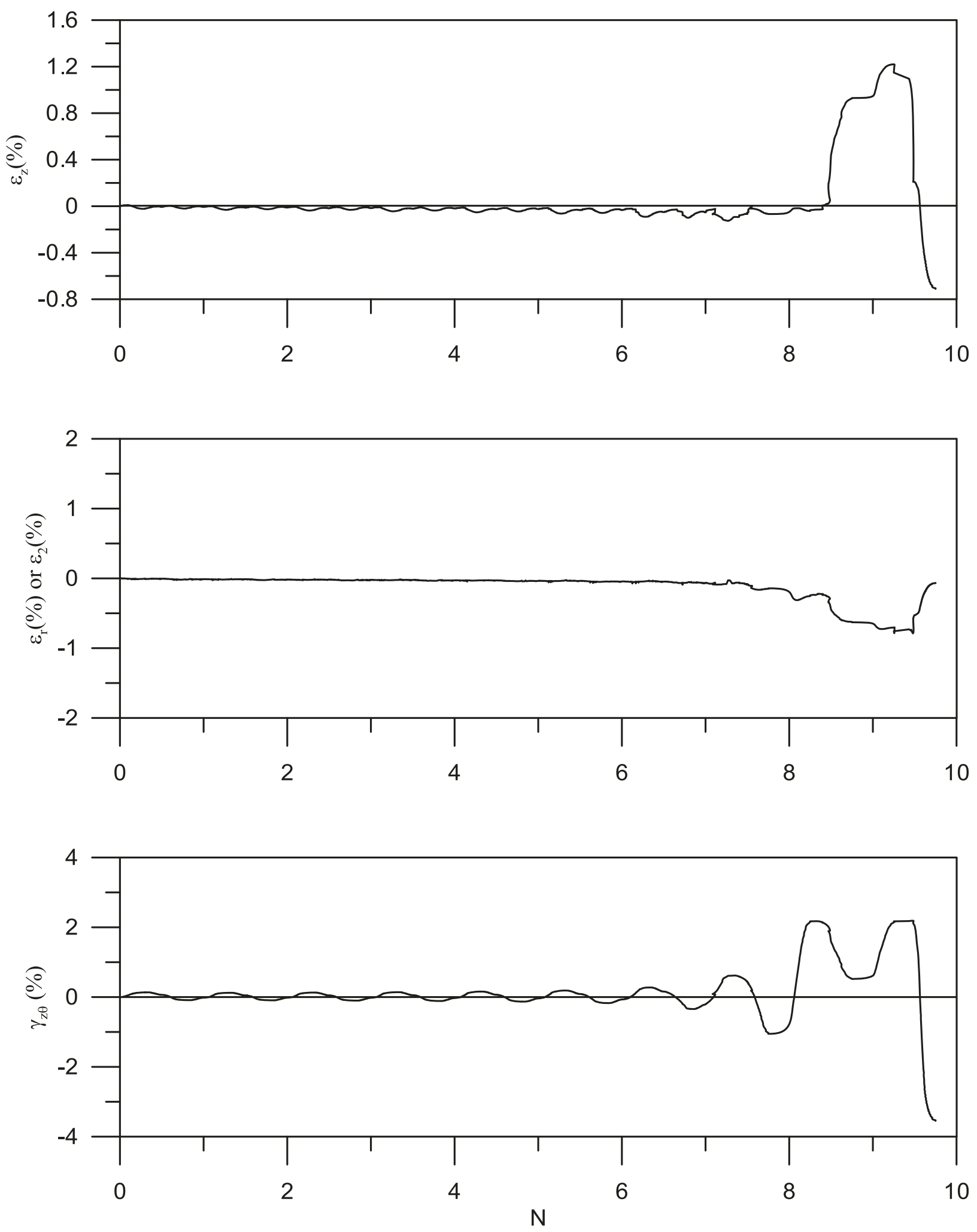

Fig. 5.12 Variation of vertical strain, radial strain and torsional shear strain applied on the horizontal plane with number of cycles for $\Delta \alpha_{\sigma}=60^{\circ}$ 
Figs. 5.13 to 5.17 show the cyclic response of Fraser River sand when the principal stress direction is smoothly rotated for a range of $\alpha_{\sigma \max }$ from $0^{\circ}$ to $90^{\circ}$ about the vertical deposition axis. The symmetry of the stress paths with respect to $\sigma_{m}^{\prime}$ is noted for all tests except the no rotation test. Plots showing the stress path for other $\alpha_{\sigma c}$ level are shown in Appendix A.

Liquefaction was not triggered even after applying 60 cycles in the test where $\alpha_{\sigma}$ is kept along the vertical deposition direction. This test is similar to a cyclic triaxial test, but only with compression loading cycles; i.e. a cyclic triaxial test with a fully rectified sine wave shear loading. This test was terminated at this stage since there is limited interest in such high number of load cycles. Excess pore pressure ration at the end of the test was about $20 \%$. The application of more cycles of loading would move the stress path gradually towards the PT line, but probably a few hundred additional load cycles would be required to trigger liquefaction. However, when the major principal stress direction was rotated by $30^{\circ}$ about the vertical axis, the soil liquefied in 14 cycles. Strain development was small during the first 12 cycles, but relatively large strains developed once the cyclic stress path reached the phase transformation line. Similarly, when $\alpha_{\sigma}$ is rotated between $-45^{\circ}$ and $+45^{\circ}$, liquefaction is induced at the 8 cycles. In each of these cases, the sand liquefied in progressively lesser number of loading cycles as the magnitude of stress rotation increased. In the test where $\alpha_{\sigma}$ is $-60^{\circ}$ and $+60^{\circ}$, liquefaction was induced in 10 cycles, and for rotation of $\alpha_{\sigma}$ between $-90^{\circ}$ and $+90^{\circ}$ liquefaction was induced at the 15 cycles. 


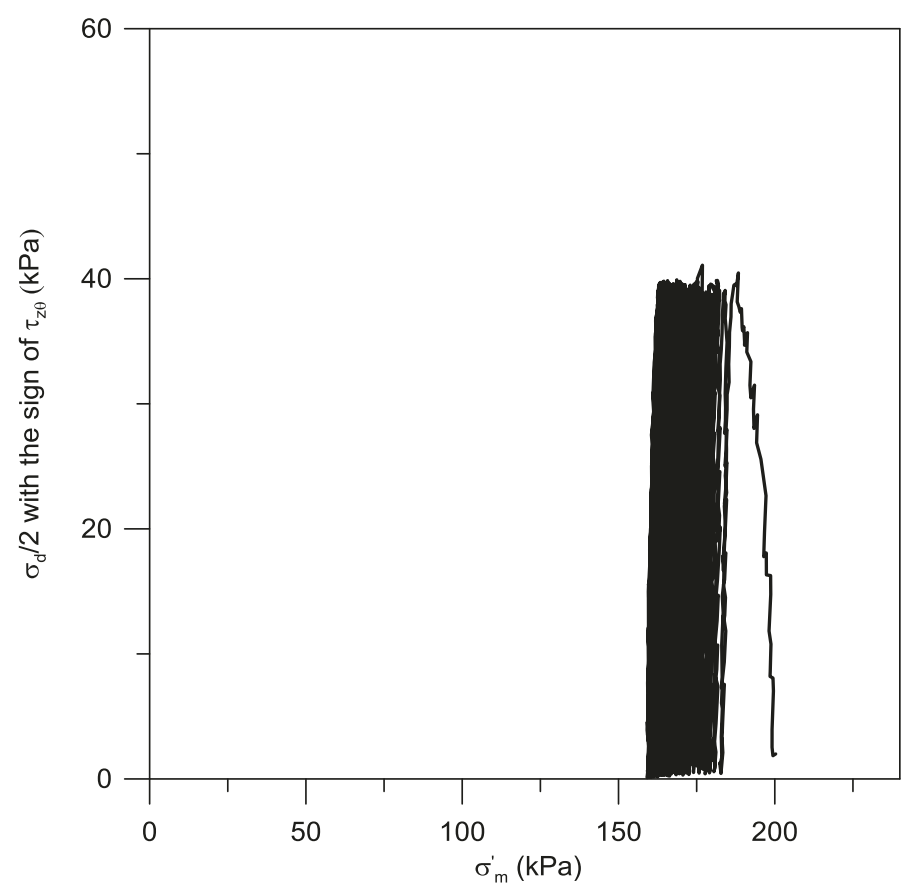

Fig 5.13 Stress path of Fraser River sand during cyclic loading of $\frac{\sigma_{d}}{2 \sigma_{3 c}^{\prime}}=0.20$ where $\alpha_{\sigma}$ was kept along vertical deposition direction

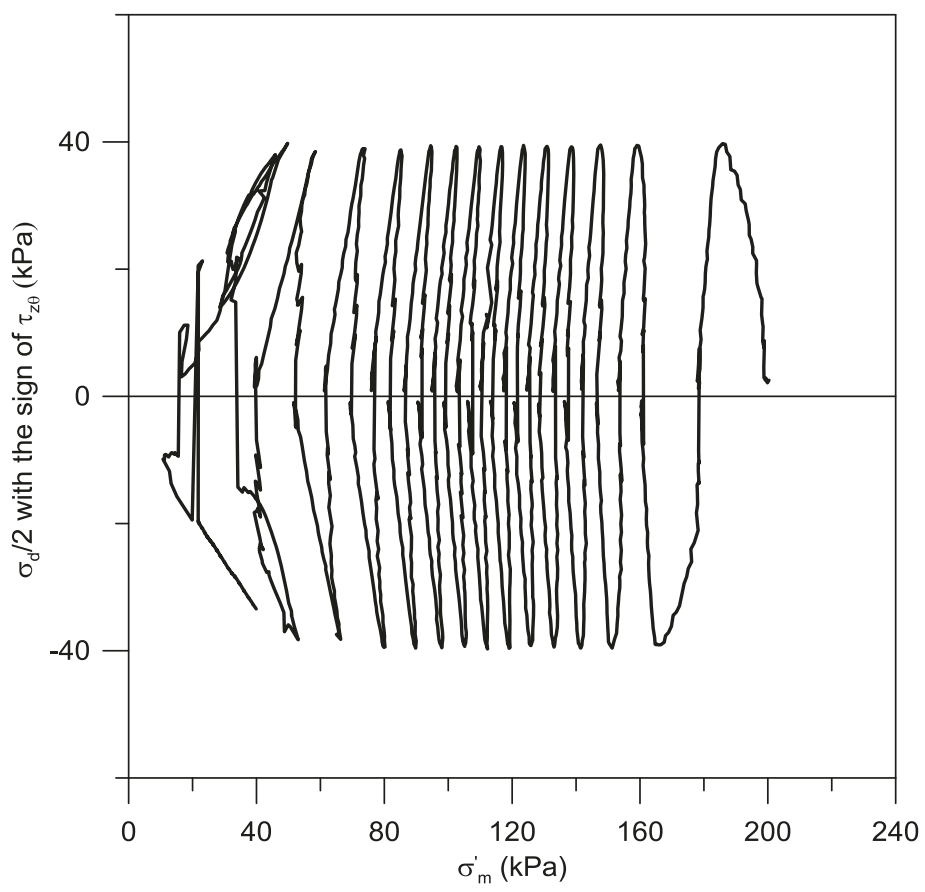

Fig 5.14 Stress path of Fraser River sand during rotation of $\alpha_{\sigma}$ between $-30^{\circ}$ and $+30^{\circ}$ 


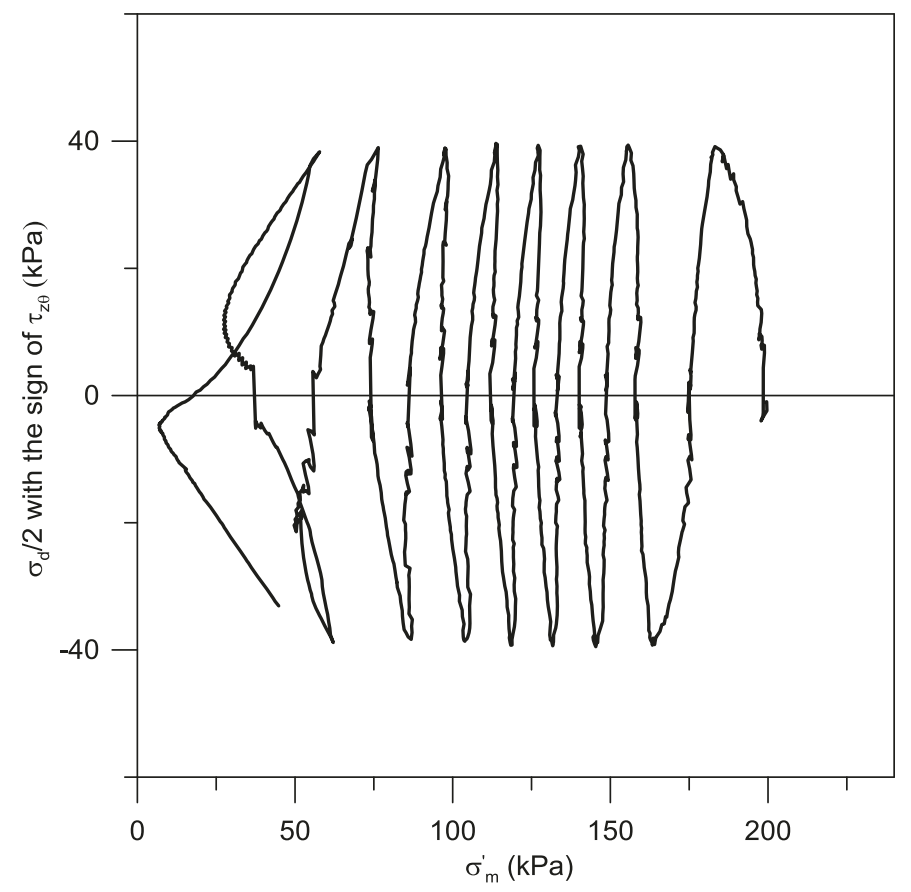

Fig 5.15 Stress path of Fraser River sand during rotation of $\alpha_{\sigma}$ between $-45^{\circ}$ and $+45^{\circ}$

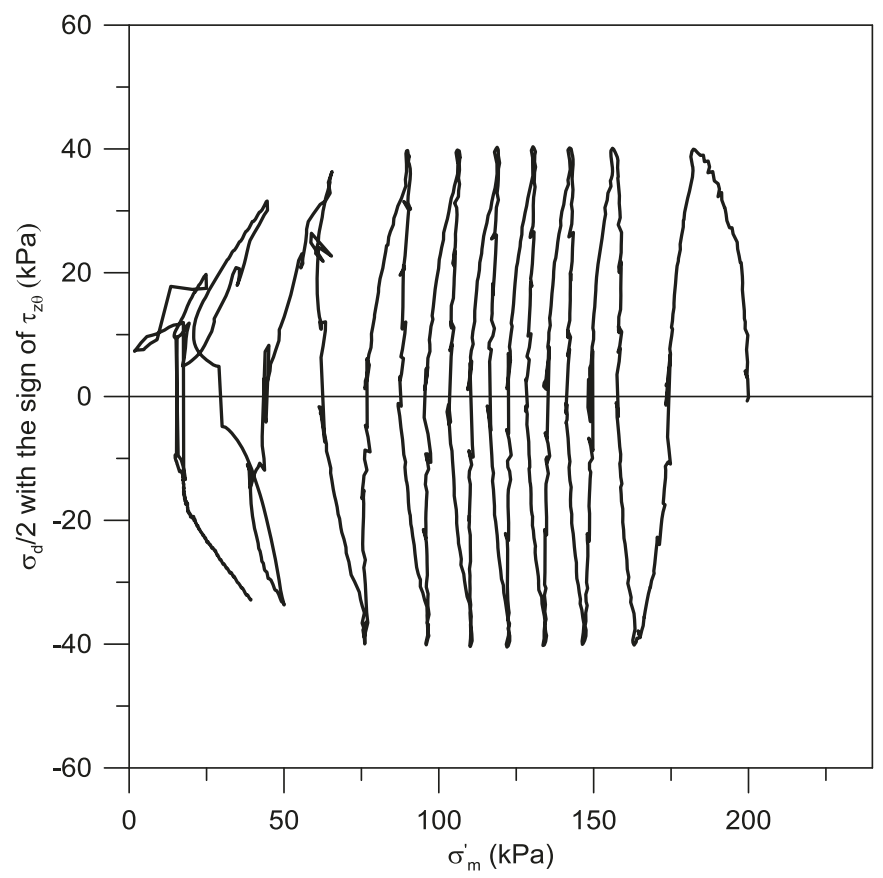

Fig 5.16 Stress path of Fraser River sand during rotation of $\alpha_{\sigma}$ between $-60^{\circ}$ and $+60^{\circ}$ 


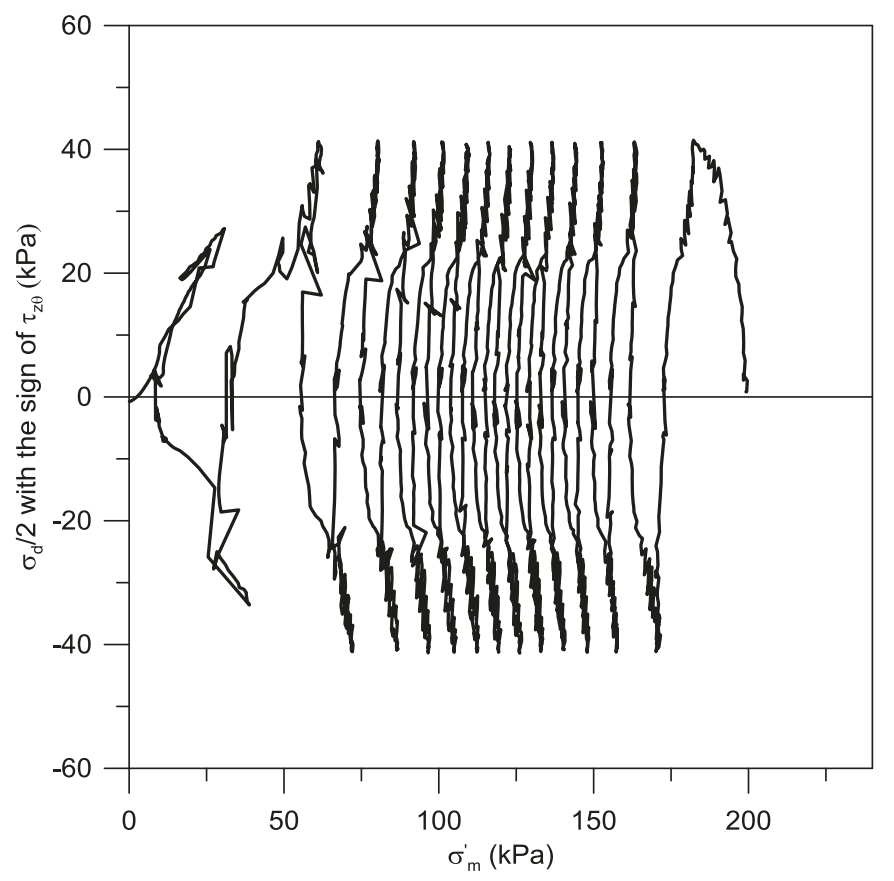

Fig 5.17 Stress path of Fraser River sand during rotation of $\alpha_{\sigma}$ between $-90^{\circ}$ and $+90^{\circ}$

Figure 5.18 compares the number of cycles to liquefaction with the degree of stress rotation. These observations indicate that the lowest cyclic resistant occurs when the principal stress rotates by $45^{\circ}$ about the vertical deposition axis. The difference in cyclic resistance between the $45^{\circ}$ and $60^{\circ}$ rotation tests is not that significant ( 8 cycles and 10 cycles). These observations are consistent with the results in the literature reported by Logeswaran (2010). 


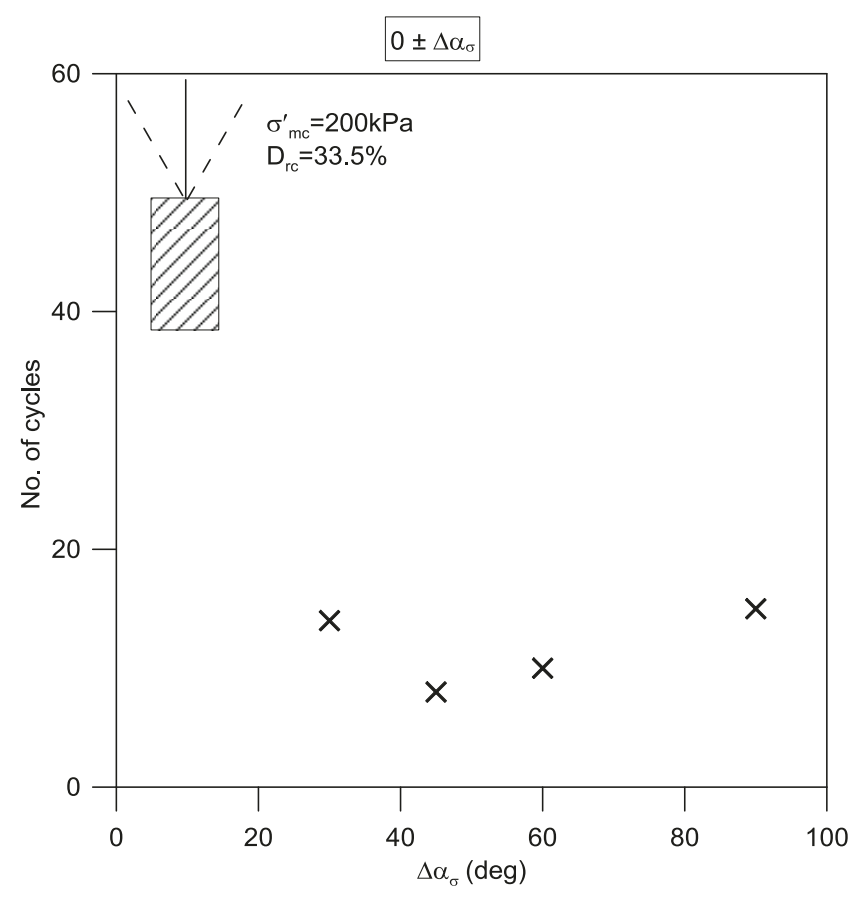

Fig. 5.18 Comparison of number of cycle required for liquefaction during $\alpha_{\sigma c}=0^{\circ}$

Fig. 5.19 illustrates the induced excess pore pressure response during cyclic loading with number of cycles as the principal stress smoothly rotated about the vertical axis. Positive excess pore pressure accumulates with increasing number of cycles. Depending of the range of $\alpha_{\sigma}$, the rate of excess pore pressure accumulation differs. The least amount of excess pore pressure is generated at the cyclic test with no rotation of $\alpha_{\sigma}$ or in other words the major principal stress is kept along the vertical deposition direction. The sand experience approximately $40 \mathrm{kPa}$ of excess pore pressure which is $20 \%$ of $\sigma_{m c}^{\prime}$ at the end of $60^{\text {th }}$ cycle. When $\alpha_{\sigma}$ is smoothly rotated between $-30^{\circ}$ and $+30^{\circ}$, the sand experiences approximately $190 \mathrm{kPa}$ of excess pore pressure which is $95 \%$ of $\sigma_{m c}^{\prime}$ at liquefaction state. During the smooth rotation of $\alpha_{\sigma}$ between $-45^{\circ}$ and $+45^{\circ}$ and $-60^{\circ}$ and $+60^{\circ}$ the sand experience the same trend of excess pore pressure response and reach an excess pore pressure of $184 \mathrm{kPa}$ which is $92 \%$ of $\sigma_{m c}^{\prime}$ and $190 \mathrm{kPa}$ which is $95 \%$ of $\sigma_{m c}^{\prime}$ at liqufaction state respectively. During smooth rotation of $\alpha_{\sigma}$ between $-90^{\circ}$ and $+90^{\circ}$ the 
sand exhibits essentially the same trend of excess pore pressure response as in the case of smooth rotation $\alpha_{\sigma}$ between $-30^{\circ}$ and $+30^{\circ}$.

The difference in excess pore pressure accumulation during no rotation $\left(\Delta \alpha_{\sigma}=0^{\circ}\right)$ and smooth rotation of $\alpha_{\sigma}$ between $-90^{\circ}$ and $+90^{\circ}$ can be attributed to the high strength of sand along the deposition direction and the lower strength along the bedding plan. Further this can be explained by the amount of maximum shear stress applied on the horizontal plan $\left(\tau_{z \theta \max }\right)$, in the no rotation case it is zero and during smooth rotation of $\alpha_{\sigma}$ between- $90^{\circ}$ and $+90^{\circ}$ it is $22.5 \mathrm{kPa}$.

During smooth rotation of $\alpha_{\sigma}$ between $-30^{\circ}$ and $+30^{\circ}$ and smooth rotation of $\alpha_{\sigma}$ between $-60^{\circ}$ and $+60^{\circ}$ the $\tau_{z \theta \max }$ is nearly equal ( $34.6 \mathrm{kPa}$ and $34.7 \mathrm{kPa}$ respectively), and the rate of excess pore pressure accumulation during these two tests can by illustrated by using the above explanation of position of principal stress refered to the deposition direction or bedding plan and the duration of imposed $\tau_{z \theta \max }$ on the specimen which is high during smooth rotation of $\alpha_{\sigma}$ between $-60^{\circ}$ and $+60^{\circ}$ compared to smooth rotation of $\alpha_{\sigma}$ between $-30^{\circ}$ and $+30^{\circ}$. These observations are consistent with the results reported by Logeswaran (2010), but for an intermediate principal stress parameter $b_{\sigma c}=0$.

Fig. 5.19 shows the variation of induced maximum shear strain $\gamma_{\max }$ during cyclic loading with smooth rotation of $\alpha_{\sigma}$. The developed $\gamma_{\max }$ is small until the stress state reaches the PT line, and significant levels of strains develop upon reaching the PT state. The test conducted with no rotation of principal stress generates only $0.16 \%$ of $\gamma_{\max }$ at the end of $60^{\text {th }}$ cycle and the test corresponding to smooth rotation of $\alpha_{\sigma}$ between $-45^{\circ}$ and $+45^{\circ}$ repersents the fastest strain accumulation. All the other scenarios fall in between these two extremes and the differences in the rate of strain accumulation can be explained by the reasons disscused to explain the excess pore pressure response. 

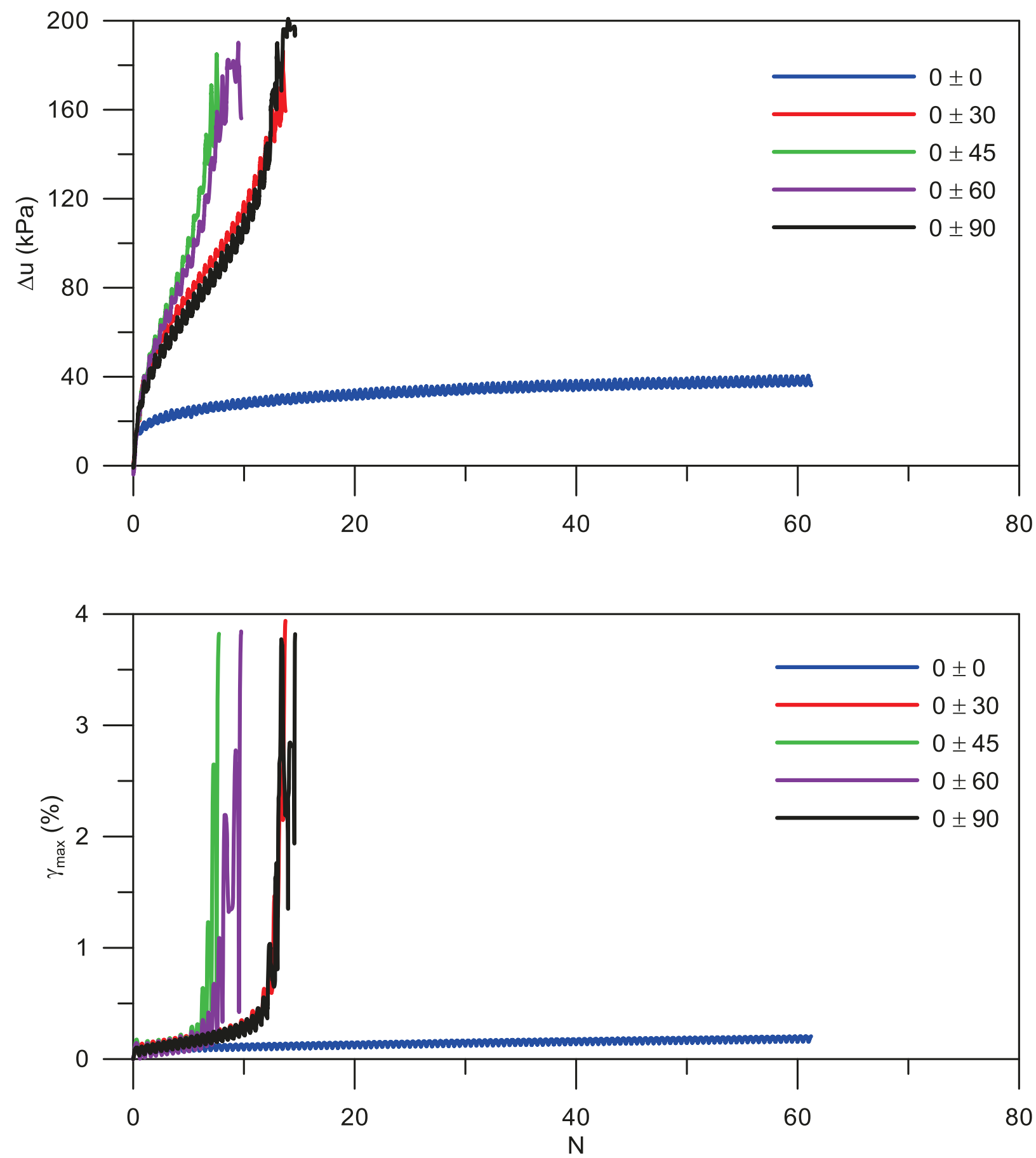

Fig. 5.19 Excess pore pressure and maximum shear strain response of Fraser River sand during cyclic loading with smooth rotation of $\alpha_{\sigma}$ from $\alpha_{\sigma c}=0^{\circ}$ 


\subsubsection{Behaviour for $\alpha_{\sigma c}=30^{\circ}$}

Fig 5.20 shows the torsional shear stress applied on the horizontal plan during smooth rotation of $\alpha_{\sigma}$ from $\alpha_{\sigma c}=30^{\circ}$. Even though $\tau_{z \theta_{\max }}$ is equal to $34.6 \mathrm{kPa}$ for the $\Delta \alpha_{\sigma}=0,30^{\circ}$ and $60^{\circ}$, their variation within cycle is different. The $\Delta \alpha_{\sigma}=0^{\circ}$ applies $34.6 \mathrm{kPa}$ of $\tau_{z \theta_{\text {max }}}$ during both half cycles whereas the $\Delta \alpha_{\sigma}=30^{\circ}$ applies $34.6 \mathrm{kPa}$ of $\tau_{z \theta_{\max }}$ only during first half cycle and $10 \mathrm{kPa}$ during the other half cycle, and the $\Delta \alpha_{\sigma}=60^{\circ}$ applies $17.3 \mathrm{kPa}$ of $\tau_{z \theta_{\max }}$ only during first half cycle and $34.6 \mathrm{kPa}$ during the other half cycle. During $\Delta \alpha_{\sigma}=45^{\circ}$ and $90^{\circ}$ tests $23.5 \mathrm{kPa}$ and $35.3 \mathrm{kPa}$ of $\tau_{z \theta_{\max }}$ applied respectively.

Fig. 5.21 shows the comparison of number of cycle required for liquefaction during different level of principal stress rotation from $\alpha_{\sigma c}=30^{\circ}$. The observations indicate that the lowest cyclic resistant occurs when the principal stress rotates $45^{\circ}$ about the initial position $\left(\alpha_{\sigma c}\right.$ $\left.=30^{\circ}\right)$.

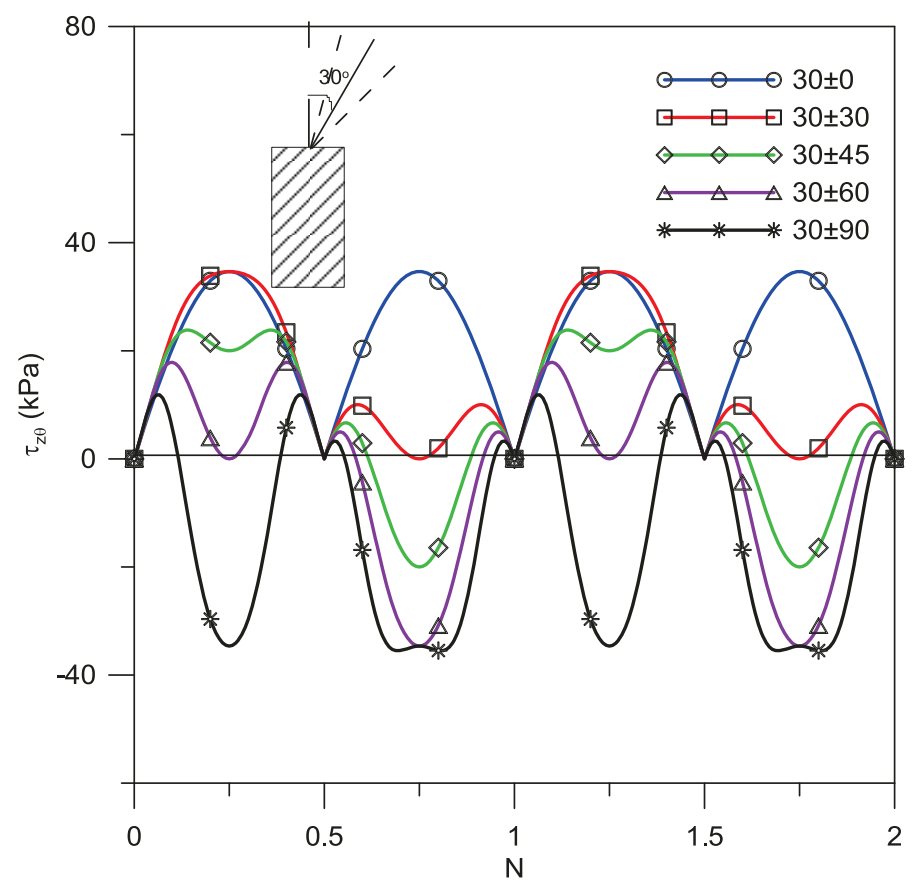

Fig 5.20 Shear stress on the horizontal plan during smooth rotation of $\alpha_{\sigma}$ from $\alpha_{\sigma c}=30^{\circ}$ 


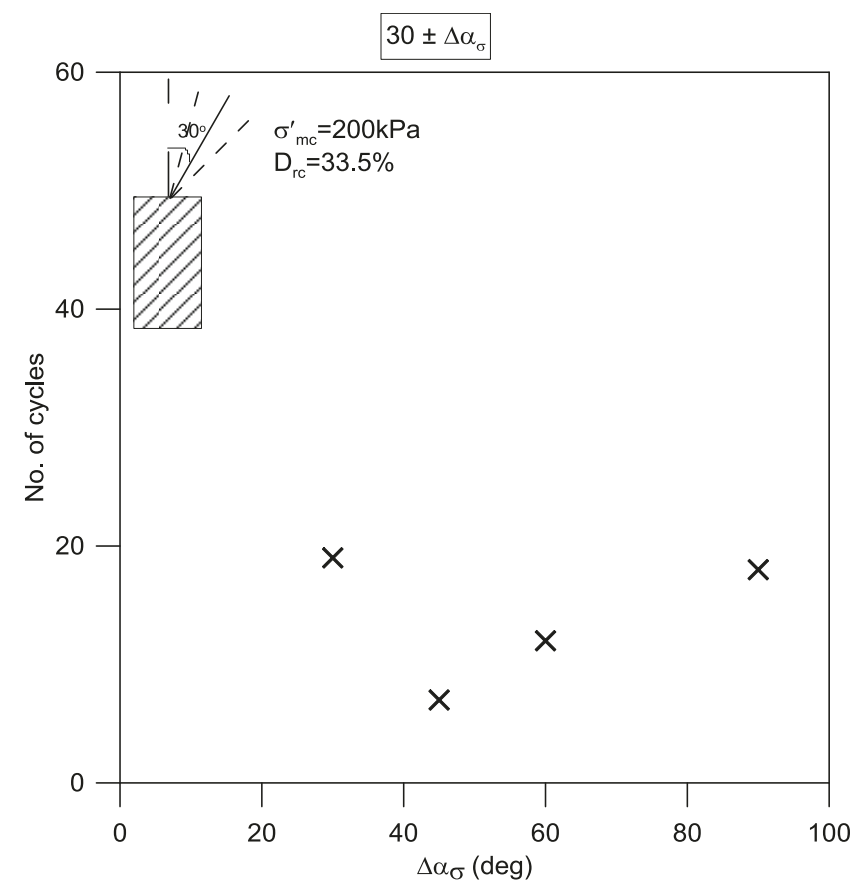

Fig. 5.21 Comparison of number of cycle required for liquefaction during $\alpha_{\sigma c}=30^{\circ}$

The excess pore pressure response shows (Fig. 5.22) that the test with $\Delta \alpha_{\sigma}=45^{\circ}$ experienced the highest rate of excess pore pressure accumulation. The lowest rate of excess pore pressure accumulation is for the test with no rotation of $\alpha_{\sigma}$ or in other words the test in which the major principal stress is kept along the initial position $\left(\alpha_{\sigma c}=30^{\circ}\right)$. The sand experiences approximately $46 \mathrm{kPa}$ of excess pore pressure which is $23 \%$ of $\sigma_{m c}^{\prime}$, at the end of $60^{\text {th }}$ cycle. Eventhough $\tau_{z \theta_{\max }}$ is equal for both $\Delta \alpha_{\sigma}=30^{\circ}$ and $\Delta \alpha_{\sigma}=60^{\circ}$ tests, the rate of excess pore pressure accumulation during $\Delta \alpha_{\sigma}=60^{\circ}$ test is higher than the rate of accumulation during the $\Delta \alpha_{\sigma}=30^{\circ}$ test. This difference in excess pore pressure accumulation can be attributed to the higher strength of the sand along the deposition direction, and to the effects of stress rotation given the anisotropy. In the test with $\Delta \alpha_{\sigma}=30^{\circ}$ the major principal stress is oriented along the vertical direction and at $\alpha_{\sigma}=60^{\circ}$ when the cyclic shear stress is at its maximum value during each half cycles. On the other hand, in the $\Delta \alpha_{\sigma}=60^{\circ}$ test the major principal stress is oriented along the bedding plane and $\alpha_{\sigma}=30^{\circ}$ at the instant of the peak shear stress. The larger 
magnitude of stress rotation, and the alignment of the major principal stress along the bedding plane contribute the larger pore water pressure generation and weaker response in the $\Delta \alpha_{\sigma}=60^{\circ}$ test. Pore water pressure generation in the $\Delta \alpha_{\sigma}=90^{\circ}$ test was marginally faster than the $\Delta \alpha_{\sigma}=$ $30^{\circ}$ test in this series of tests (when stress rotation was about an axis inclined at $30^{\circ}$ to vertical). $\Delta \alpha_{\sigma}=90^{\circ}$ test invlolves much larger stress rotation, and the peak cyclic shear stresses occur when major principal stress direction is inclined at $\alpha_{\sigma}=60^{\circ}$ during each half cycles compared to at $\alpha_{\sigma}=0^{\circ}$ and $60^{\circ}$ in the $\alpha_{\sigma c}=30^{\circ}$ test.

Even though $\tau_{z \theta_{\max }}$ is almost equal, the rate of excess pore pressure accumulation during rotation of $\Delta \alpha_{\sigma}=90^{\circ}$ is much higher than the test with no change in the major principal stress direction. This observation may be explained by the nature of major principal stress rotation. For the $90^{\circ}$ rotation major principal stress crosses the weakest bedding plane and the strongest deposition direction alternately whereas for the no rotation case fixed at $30^{\circ}$ and no such crossing occurs or in other words no stress reversal occurs during the no rotation case where as stress reversal occurs during the $90^{\circ}$ rotation case where compression and extention mode of loading is applied alternately. This kind of behaviour is already noted for the $\alpha_{\sigma c}=0^{\circ}$ and $\Delta \alpha_{\sigma}=0^{\circ}$ case.

The variation of the maximum shear strain $\left(\gamma_{\max }\right)$ developed with number of loading cycles shows (Fig. 5.22) that the test conducted with no rotation of principal stress generates only $0.12 \%$ of $\gamma_{\max }$ at the end of $60^{\text {th }}$ cycle. The test corresponding to smooth rotation of $\alpha_{\sigma}=$ $45^{\circ}$ repersents the fastest strain accumulation. All the other scenarios fall in between these two extreams and difference in the rate of accumulation can be explained by the reasons disscused to explain the excess pore pressure response. 

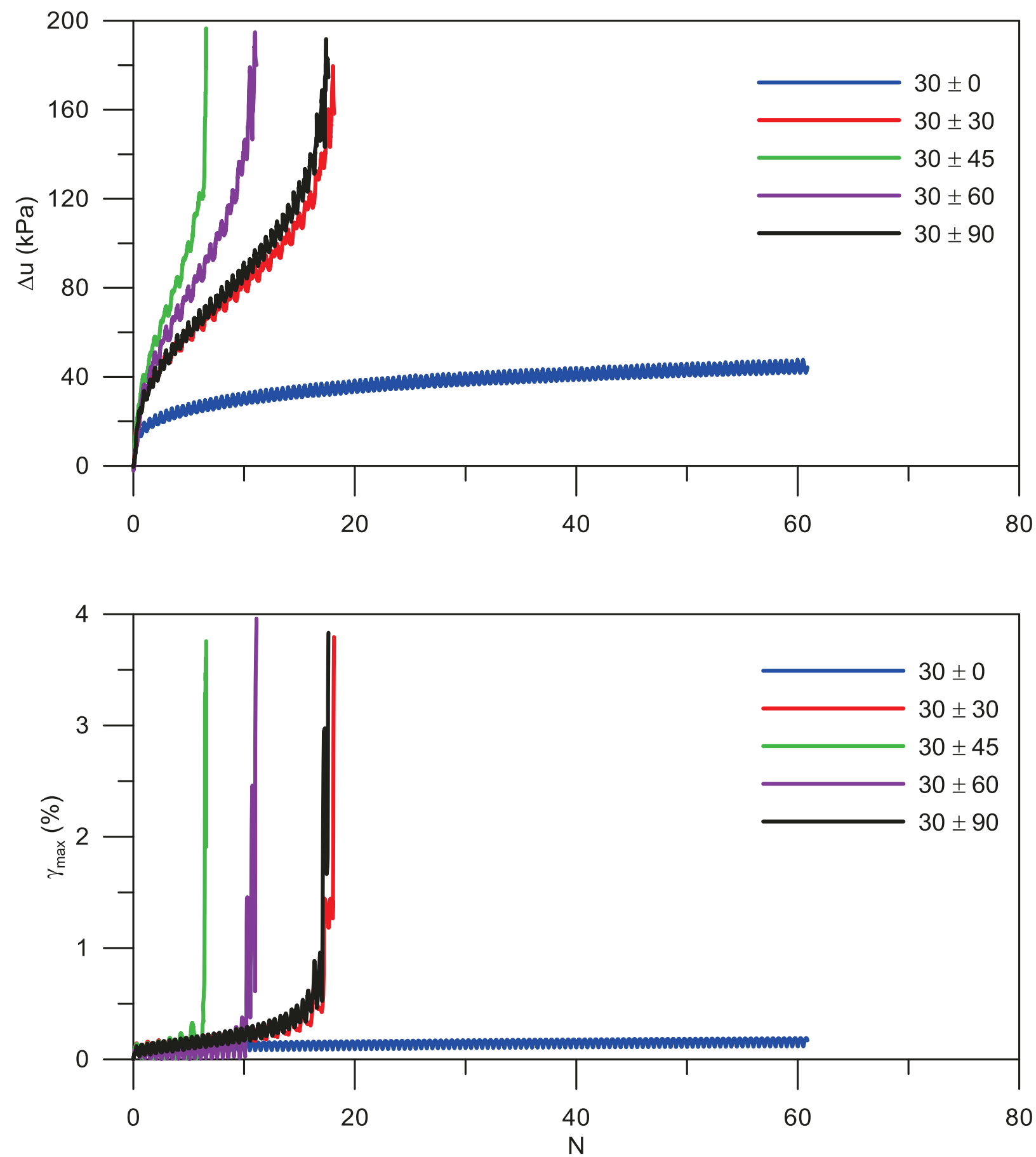

Fig. 5.22 Excess pore pressure and maximum shear strain response of Fraser River sand during cyclic loading with smooth rotation of $\alpha_{\sigma}$ from $\alpha_{\sigma c}=30^{\circ}$ 


\subsubsection{Behaviour for $\alpha_{\sigma c}=45^{\circ}$}

Fig 5.23 shows the torsional shear stress applied on the horizontal plane during smooth rotation of $\alpha_{\sigma}$ from $\alpha_{\sigma c}=45^{\circ}$. Since the major principal stress at the instant of peak cyclic stress is orientated at $45^{\circ}$ from horizontal plane for $\Delta \alpha_{\sigma}=0^{\circ}$ and $90^{\circ}$, the maximum torsional shear stress, $\tau_{z} \theta_{\max }$ required is the same in both cases $(40 \mathrm{kPa})$ but their variation within the cycle is different as seen in the Figure. During the $\Delta \alpha_{\sigma}=0^{o}$ test both half cycles applies $40 \mathrm{kPa}$ of $\tau_{z \theta_{\max }}$ whereas during the $\Delta \alpha_{\sigma}=90^{\circ}$ test, the compression cycle applies only $7.1 \mathrm{kPa}$, and the extension cycle applies $40 \mathrm{kPa}$ of $\tau_{z \theta_{\max }}$. In comparison, $\tau_{z \theta_{\max }}$ during the $\Delta \alpha_{\sigma}=30^{\circ}, 45^{\circ}$ and $60^{\circ}$ tests is $21.3 \mathrm{kPa}, 14.1 \mathrm{kPa}$ and $20 \mathrm{kPa}$ respectively.

Fig. 5.24 shows a comparison of the number of cycle required for liquefaction during different level of principal stress rotation from $\alpha_{\sigma c}=45^{\circ}$. The observations indicate that the lowest cyclic resistant occurs when the principal stress rotates by $45^{\circ}$ about the initial position $\left(\alpha_{\sigma c}=45^{\circ}\right)$

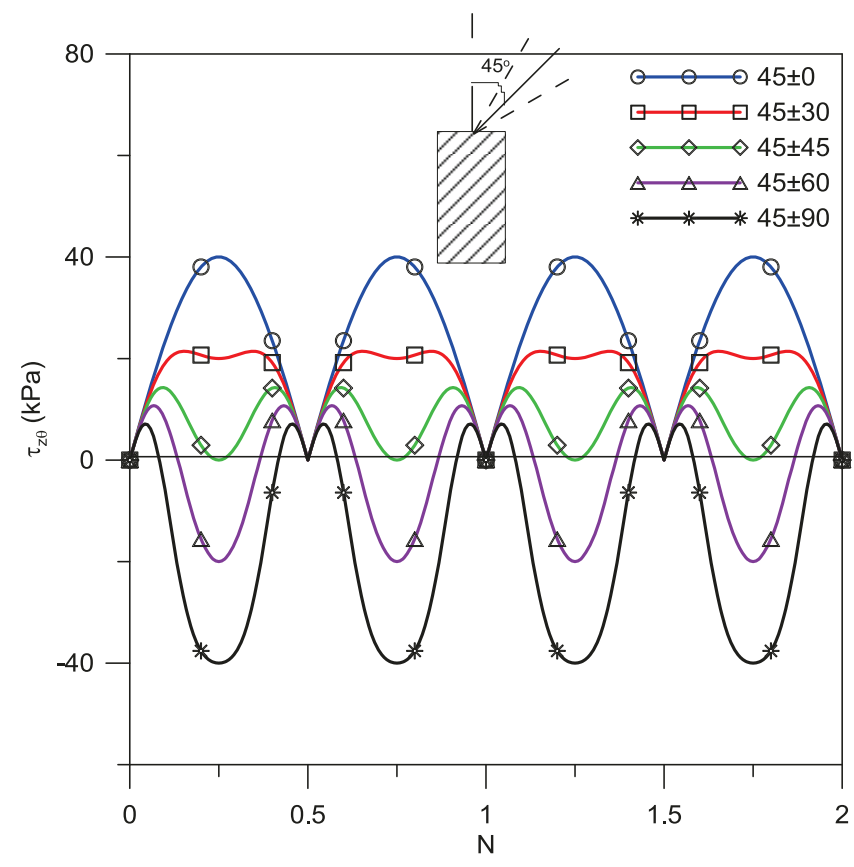

Fig 5.23 Shear stress on the horizontal plan during smooth rotation of $\alpha_{\sigma}$ from $\alpha_{\sigma c}=45^{\circ}$ 


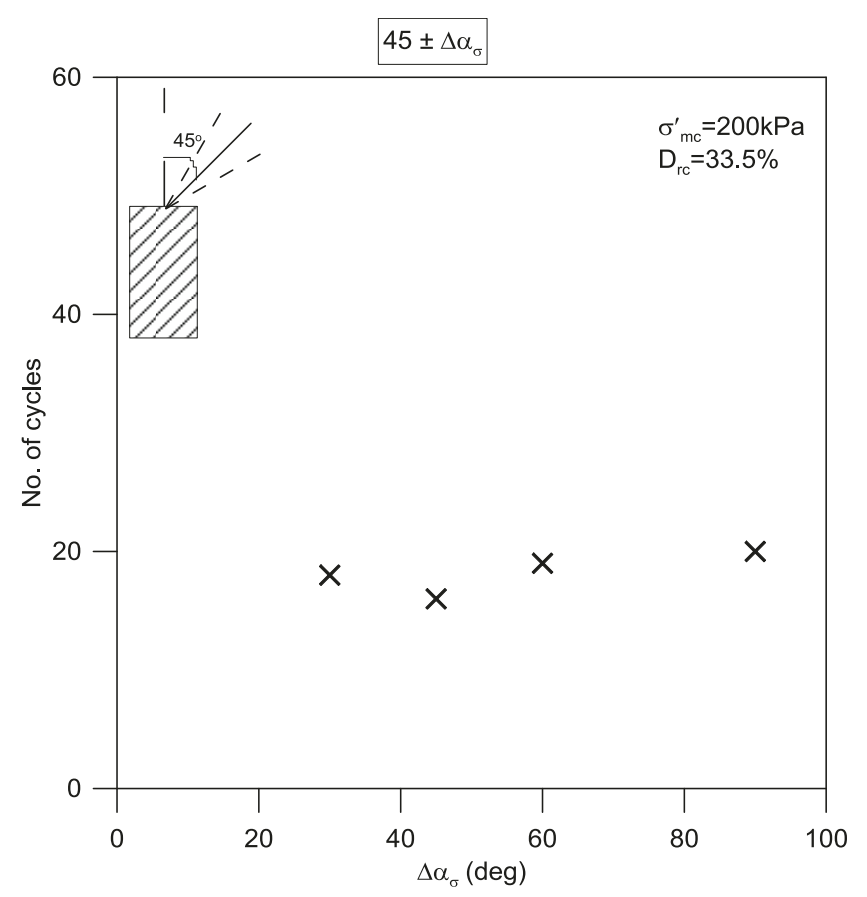

Fig. 5.24 Comparison of number of cycle required for liquefaction during $\alpha_{\sigma c}=45^{\circ}$

The excess pore pressure response shows (Fig. 5.25) that the $\Delta \alpha_{\sigma}=45^{\circ}$ test experiences the highest rate of excess pore pressure accumulation. The lowest rate of excess pore pressure is accumulated for the test with no rotation of $\alpha_{\sigma}$ (i.e. when the major principal stress is kept along the initial position at $\alpha_{\sigma c}=45^{\circ}$ ). The sand experiences approximately $40 \mathrm{kPa}$ of excess pore pressure which is $20 \%$ of $\sigma_{m c}^{\prime}$ at the end of the $60^{\text {th }}$ cycle. It is noted that $\tau_{z \theta_{\text {max }}}$ is nearly equal in both the $\Delta \alpha_{\sigma}=30^{\circ}$ and the $\Delta \alpha_{\sigma}=60^{\circ}$ tests. The rate of accumulation of excess pore pressure is approximately same in these two tests since the peak cyclic stress occurs at inclinations of $\alpha_{\sigma}=15^{\circ}$ and $75^{\circ}$ in both tests. On the other hand, even though the $\tau_{z \theta_{\text {max }}}$ is equal in the $\Delta \alpha_{\sigma}=$ $0^{\circ}$ (no rotation) and the $\Delta \alpha_{\sigma}=90^{\circ}$ tests, the rate of excess pore pressure accumulation during the $\Delta \alpha_{\sigma}=90^{\circ}$ test is higher than the rate of excess pore pressure accumulation during no rotation because of the significant differences in stress rotation. During the $\Delta \alpha_{\sigma}=90^{\circ}$ test major principal stress crosses the weakest bedding plane and the strongest deposition direction alternately, where as for the no rotation case $\left(\Delta \alpha_{\sigma}=0^{\circ}\right)$ no such crossing occurs. In other words 
no stress reversal occurs during the no rotation case where as stress reversal occurs during the $90^{\circ}$ rotation test causes compression and extention mode of loading applied alternately. As noted earlier, the variation of shear strain development follows that of the excess pore pressure development (Fig. 5.25). The test conducted with no rotation $\left(\Delta \alpha_{\sigma}=0^{\circ}\right)$ of principal stress generates only $0.43 \%$ of $\gamma_{\max }$ at the end of $60^{\text {th }}$ cycle. The test corresponding to $\Delta \alpha_{\sigma}=45^{\circ}$ repersents the fastest strain accumulation.
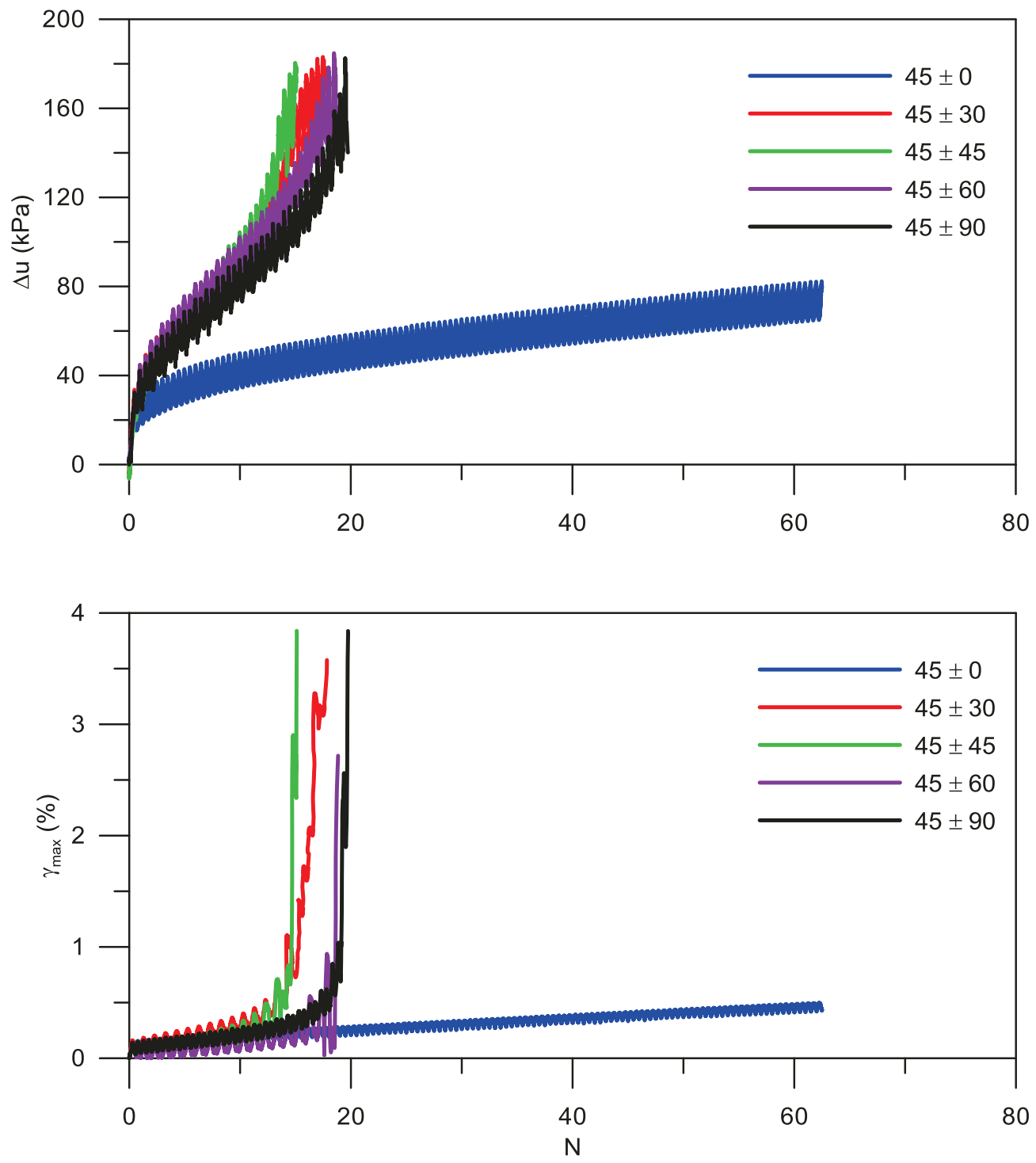

Fig. 5.25 Excess pore pressure and maximum shear strain response of Fraser River sand during cyclic loading with smooth rotation of $\alpha_{\sigma}$ from $\alpha_{\sigma c}=45^{\circ}$ 


\subsubsection{Behaviour for $\alpha_{\sigma c}=60^{\circ}$}

Fig 5.26 shows the torsional shear stress applied on the horizontal plan during smooth rotation of $\alpha_{\sigma}$ from $\alpha_{\sigma c}=60^{\circ}$. Even though $\tau_{z \theta_{\max }}$ is equal to $34.6 \mathrm{kPa}$ for the $\Delta \alpha_{\sigma}=0,30^{\circ}$ and $60^{\circ}$, the variation within the load cycle is different. The $\Delta \alpha_{\sigma}=0^{\circ}$ test applies $34.6 \mathrm{kPa}$ of $\tau_{z \theta_{\max }}$ during both half cycles whereas the $\Delta \alpha_{\sigma}=30^{\circ}$ applies $10 \mathrm{kPa}$ of $\tau_{z \theta_{\max }}$ only during first half cycle and $34.6 \mathrm{kPa}$ during the other half cycle. The $\Delta \alpha_{\sigma}=60^{\circ}$ test applies $34.6 \mathrm{kPa}$ of $\tau_{z \theta_{\max }}$ only during first half cycle and $17.3 \mathrm{kPa}$ during the other half cycle. The $\tau_{z \theta_{\text {max }}}$ during the $\Delta \alpha_{\sigma}=45^{\circ}$ and $90^{\circ}$ tests is $23.5 \mathrm{kPa}$ and $35.3 \mathrm{kPa}$ respectively.

Fig. 5.27 shows the comparison of number of cycle required for liquefaction during different level of principal stress rotation from $\alpha_{\sigma c}=45^{\circ}$. Similar to the previous cases, test results indicate that the lowest cyclic resistance occurs when the principal stress rotates by $45^{\circ}$ about the initial position $\left(\alpha_{\sigma c}=60^{\circ}\right)$.

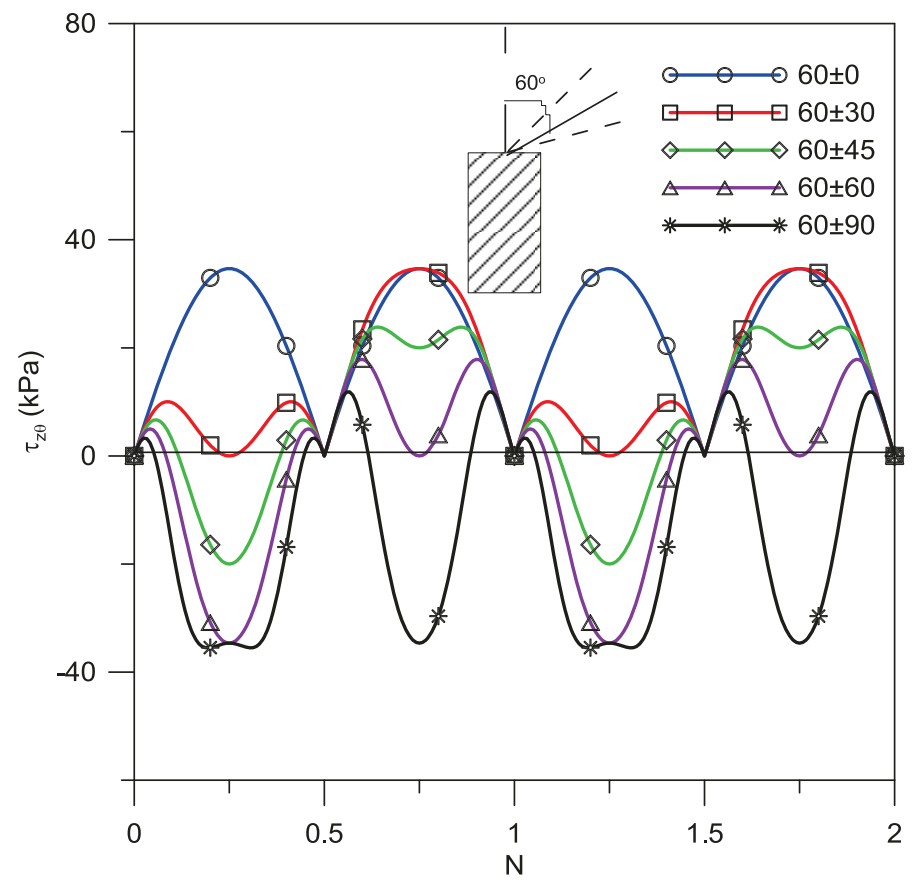

Fig 5.26 Shear stress on the horizontal plan during smooth rotation of $\alpha_{\sigma}$ from $\alpha_{\sigma c}=60^{\circ}$ 


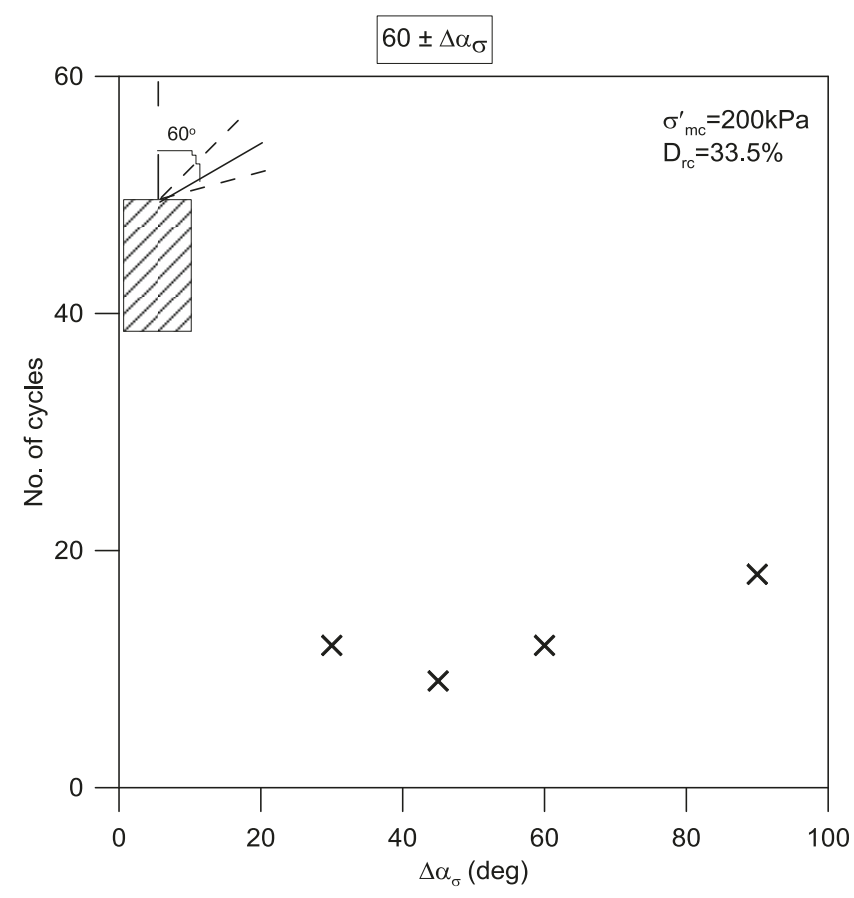

Fig. 5.27 Comparison of number of cycle required for liquefaction during $\alpha_{\sigma c}=60^{\circ}$

The excess pore pressure response shows (Fig. 5.28) that the $\Delta \alpha_{\sigma}=45^{\circ}$ test experiences the heighest rate of excess pore pressure accumulation. The lowest rate of excess pore pressure is accumulated for the test with no rotation of $\alpha_{\sigma}$ (i.e. when the major principal stress is kept along the initial position at $\alpha_{\sigma c}=60^{\circ}$ ). The sand experiences approximately $46 \mathrm{kPa}$ of excess pore pressure which is $23 \%$ of $\sigma_{m c}^{\prime}$ at the end of $60^{\text {th }}$ cycle. As before, $\tau_{z \theta_{\max }}$ is equal in both $\Delta \alpha_{\sigma}=30^{\circ}$ and $\Delta \alpha_{\sigma}=60^{\circ}$ tests. However, the rate of strain/excess pore pressure accumulation during $\Delta \alpha_{\sigma}=30^{\circ}$ is higher than the rate of accumulation in the $\Delta \alpha_{\sigma}=60^{\circ}$ test. This difference can be attributed to the higher strength of the sand along the deposition direction. $\Delta \alpha_{\sigma}=30^{\circ}$ test aligns the major principal stress along $\alpha_{\sigma}=30^{\circ}$ and $90^{\circ}$ (weakest bedding plane) at the instant of the maximum cyclic shear stress but $\Delta \alpha_{\sigma}=60^{\circ}$ test aligns the major principal stress along $\alpha_{\sigma}=0^{\circ}$ (strongest deposition direction) and $60^{\circ}$. Even though $\tau_{z \theta_{\max }}$ is almost equal during the no rotation $\left(\Delta \alpha_{\sigma}=0^{\circ}\right)$ and $\Delta \alpha_{\sigma}=90^{\circ}$ test, the rate of excess pore pressure accumulation is 
higher in the $\Delta \alpha_{\sigma}=90^{\circ}$ test due to stress rotation. In the $\Delta \alpha_{\sigma}=90^{\circ}$ test, the major principal stress crosses the weakest bedding plane and the strongest deposition direction alternately whereas for the stress directions are fixed at $60^{\circ}$ and no such crossing occurs in the $\Delta \alpha_{\sigma}=0^{\circ}$ test. The variation of induced maximum shear strain $\left(\gamma_{\max }\right)$ shows (Fig. 5.28) that the test conducted with no rotation of principal stress generates only $0.26 \%$ of $\gamma_{\max }$ at the end of $60^{\text {th }}$ cycle. The test corresponding to smooth rotation of $\Delta \alpha_{\sigma}=45^{\circ}$ repersents the fastest strain accumulation. All the other scenarios fall in between these two extremes as noted in the previous series.
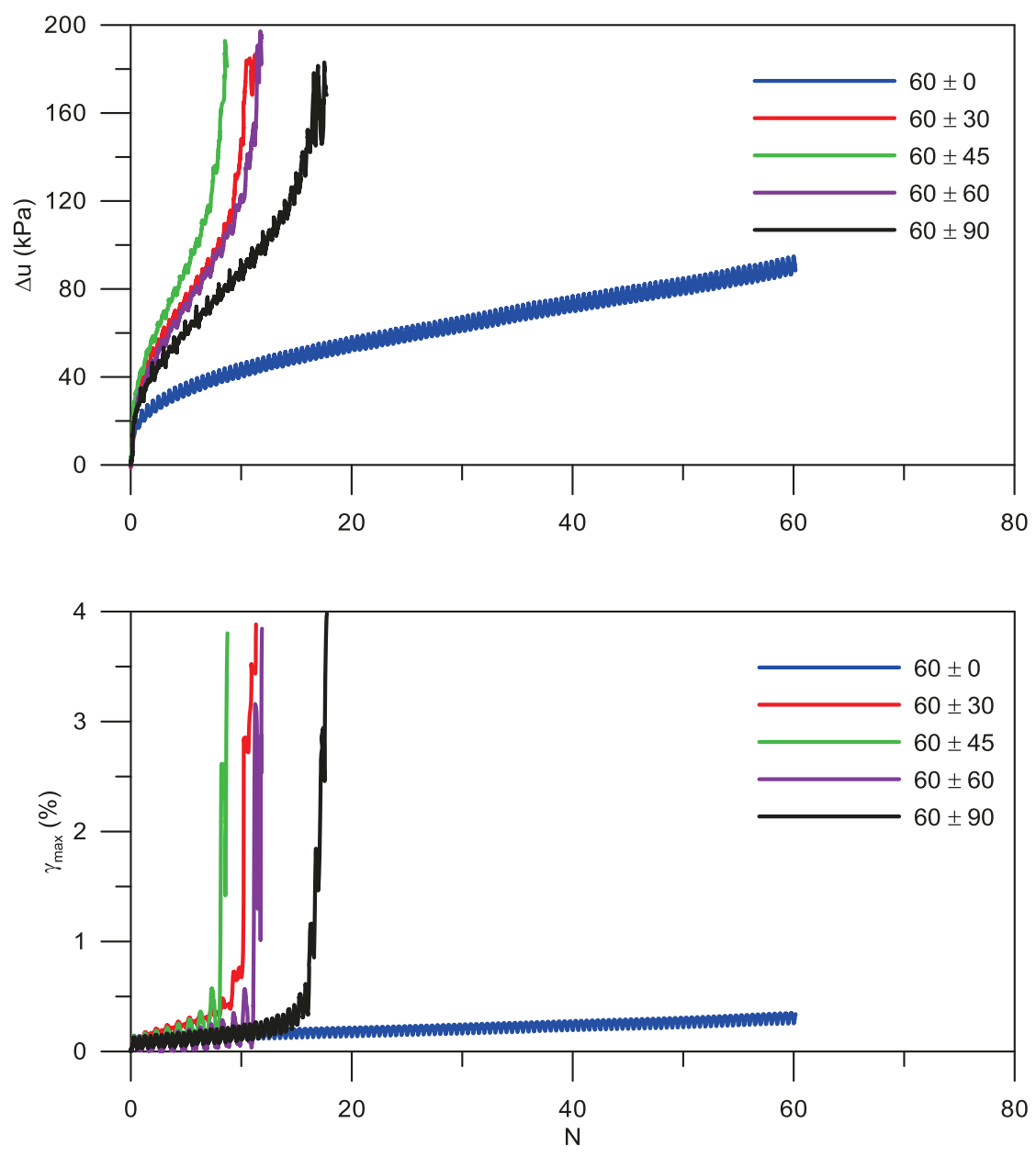

Fig. 5.28 Excess pore pressure and maximum shear strain response of Fraser River sand during cyclic loading with smooth rotation of $\alpha_{\sigma}$ from $\alpha_{\sigma c}=60^{\circ}$ 


\subsubsection{Behaviour for $\alpha_{\sigma c}=90^{\circ}$}

Fig 5.29 shows the torsional shear stress applied on the horizontal plan during smooth rotation of $\alpha_{\sigma}$ from $\alpha_{\sigma c}=90^{\circ}$. Since the major principal stress is oriented along the horizontal bedding plane during the $\Delta \alpha_{\sigma}=0^{\circ}$, there is no torsional shear stress applied on the horizontal plane. The $\tau_{z \theta_{\text {max }}}$ in the $\Delta \alpha_{\sigma}=30^{\circ}$ and $60^{\circ}$ tests is almost equal but the variation within the cycle is different. The $\Delta \alpha_{\sigma}=45^{\circ}$ test causes symmetrical loading about the horizontal plane, and requires the maximum torsional shear stress amplitude of $\tau_{z \theta_{\max }}=40 \mathrm{kPa}$, whereas the $\Delta \alpha_{\sigma}=90^{\circ}$ test requires a $\tau_{z \theta_{\max }}=22.5 \mathrm{kPa}$.

Fig. 5.30 shows the comparison of number of cycle required for liquefaction during different level of principal stress rotation from $\alpha_{\sigma c}=90^{\circ}$. As in the previous series of tests, test results indicate that the lowest cyclic resistance occurs when the principal stress rotates by $45^{\circ}$ about the initial position of $\alpha_{\sigma c}=90^{\circ}$.

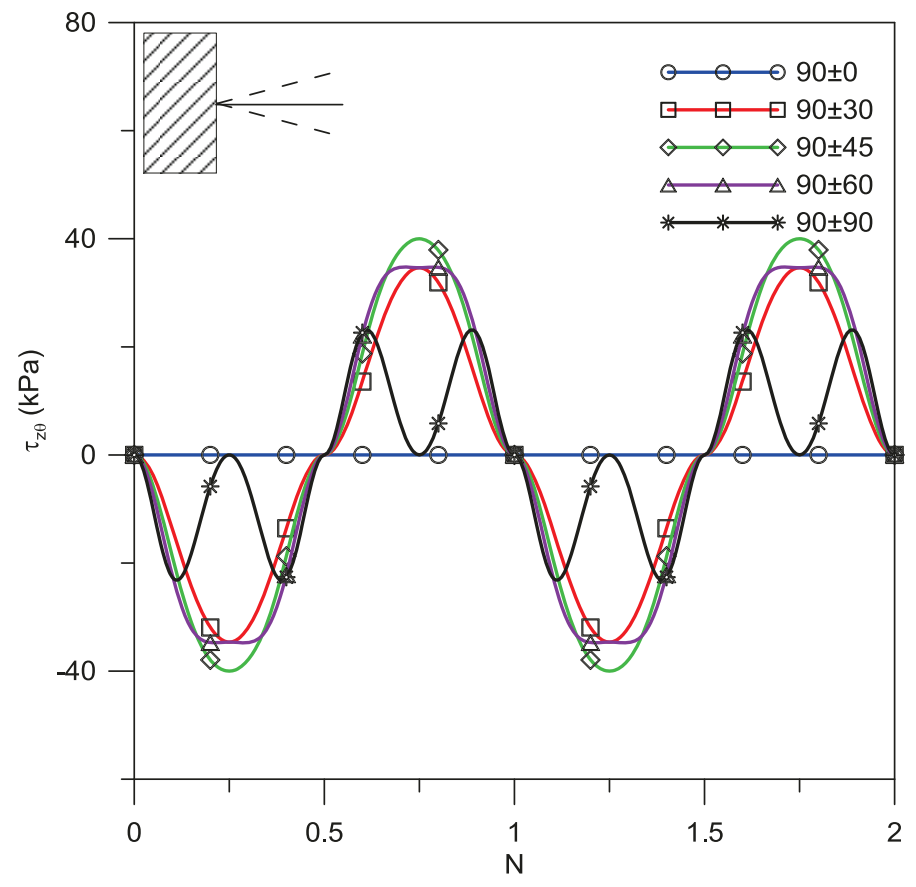

Fig 5.29 Shear stress on the horizontal plan during smooth rotation of $\alpha_{\sigma}$ from $\alpha_{\sigma c}=90^{\circ}$ 


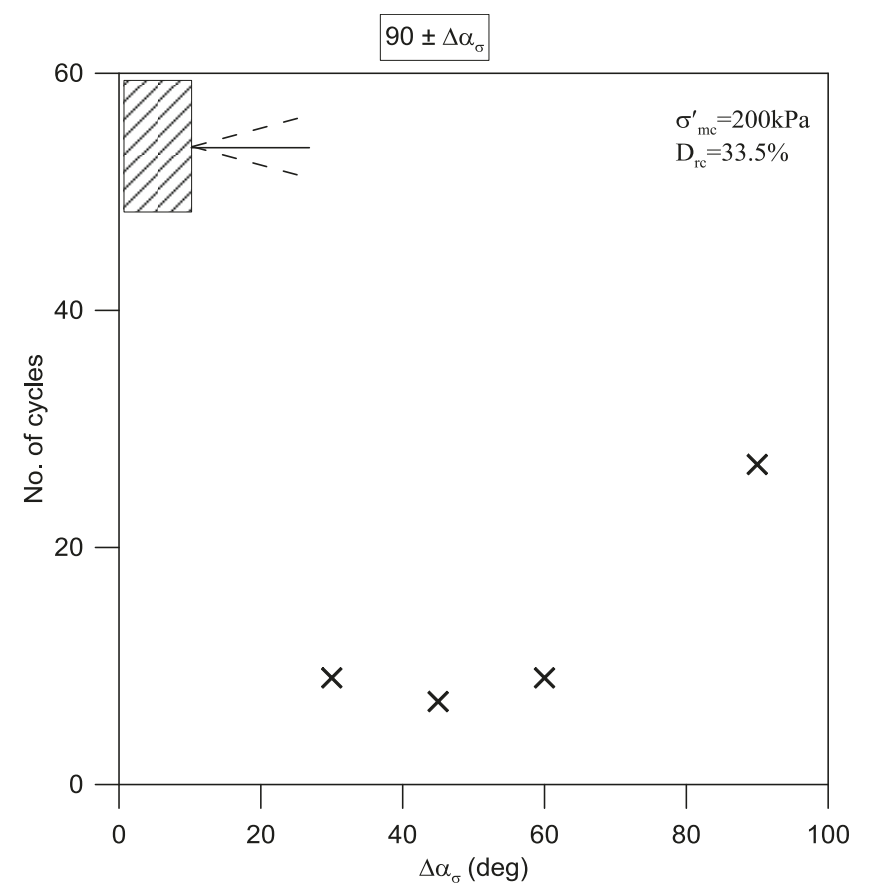

Fig. 5.30 Comparison of number of cycle required for liquefaction during $\alpha_{\sigma c}=90^{\circ}$

Fig. 5.31 shows that the excess pore pressure and strain development responses are similar to those those observed in the earlier series of tests. The $\Delta \alpha_{\sigma}=45^{\circ}$ test experiences the highest rate of excess pore pressure accumulation, and the the test with no rotation of $\alpha_{\sigma}$ (i.e. when the major principal stress is kept along the bedding plane) experiences the lowest rate of pore pressure/strain accumulation. The sand experience approximately $52 \mathrm{kPa}$ of excess pore pressure which is $26 \%$ of $\sigma_{m c}^{\prime}$ at the end of $60^{\text {th }}$ cycle when there was no stress rotation, but it generated over $90 \%$ of excess pore pressure in the other tests (those involving stress rotation) and liquefied from 7 to 27 cycles depening on the degree of stress rotation. Even though $\tau_{z \theta_{\max }}$ is almost equal for both $\Delta \alpha_{\sigma}=30^{\circ}$ and $\Delta \alpha_{\sigma}=60^{\circ}$ tests, the test with $\Delta \alpha_{\sigma}=30^{\circ}$ rotation shows somewhat faster pore water pressure generation. This can be attributed to the orientation of the major principal stress at the instant of the peak cyclic shear stress as in the earlier cases $\left(\alpha_{\sigma c}=\right.$ $60^{\circ}$ in the $\Delta \alpha_{\sigma}=30^{\circ}$ test as opposed to $\alpha_{\sigma c}=30^{\circ}$ in the $\Delta \alpha_{\sigma}=60^{\circ}$ test). However, the 
differences have been minor, and eventually both of them liquefied at the same number of cycles which demonstrates the effect of the additional stress rotation. As noted in each of the earlier series of tests, rate of pore water pressure and strain development is higher in the $\Delta \alpha_{\sigma}=90^{\circ}$ test compared to the $\Delta \alpha_{\sigma}=0^{\circ}$ (no rotation) test.

Only about $0.33 \%$ maximum shear strain $\left(\gamma_{\max }\right)$ was mobilized after 60 cycles of loading at $\operatorname{CSR}=0.20$ when there was no stress rotation (Fig. 5.31). However, the specimen liquefied (i.e., generated in excess of $3.75 \%$ shear strain) under all other conditions within about 27 cycles, and the test corresponding to $\Delta \alpha_{\sigma}=45^{\circ}$ was the weakest.
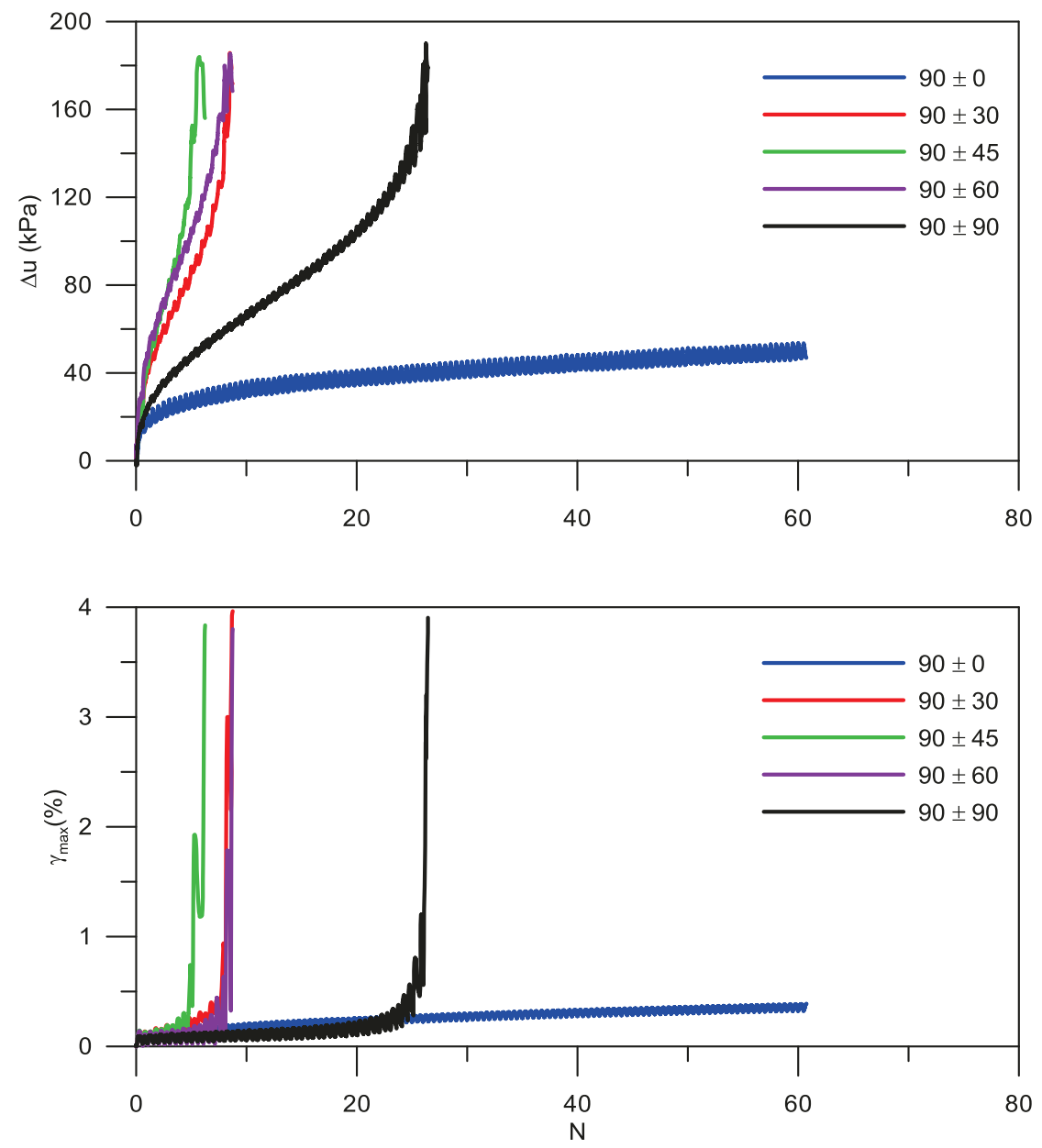

Fig. 5.31 Excess pore pressure and maximum shear strain response of Fraser River sand during cyclic loading with smooth rotation of $\alpha_{\sigma}$ from $\alpha_{\sigma c}=90^{\circ}$ 


\subsection{INFLUENCE OF $\boldsymbol{\alpha}_{\sigma c}$ ON CYCLIC BEHAVIOUR OF SAND}

Test results are presented in order to study the effect of direction of major principal stress at the end of consolidation $\left(\alpha_{\sigma c}\right)$ on the liquefaction resistance of sand during cyclic loading at a fixed cyclic stress ratio of 0.20 . Test results of specimens subjected to a given amount of principal stress rotation (about different axis) are compared in this section. The observations indicate that the cyclic resistance of sands at a given CSR (defined as a ratio of the maximum cyclic shear stress to the effective normal stress) depends on the magnitude of the maximum torsional shear stress applied on the horizontal plane $\tau_{z \theta_{\max }}$. Plots showing the development of maximum shear strain and excess pore pressure with number of loading cycles at each $\Delta \alpha_{\sigma}$ level are shown in Appendix B.

\subsubsection{Behaviour for $\Delta \alpha_{\sigma}=0^{\circ}$}

Fig. 5.32 shows the variation of torsional shear stress $\tau_{z \theta}$ applied on the horizontal plane of the specimen when the cyclic loading was applied along fixed inclinations of principal stresses. These tests have been termed $\Delta \alpha_{\sigma}=0^{\circ}$ tests in the previous section, and represent the effect of the principal stress directions on the cyclic response of soils. Five tests at principal stress directions of $\alpha_{\sigma c}=0^{\circ}, 30^{\circ}, 45^{\circ}, 60^{\circ}$ and $90^{\circ}$ were conducted. No torsional shear stress $\tau_{z \theta}$ is required for the first and last cases $\left(\alpha_{\sigma c}=0^{\circ}\right.$ and $\left.90^{\circ}\right)$ and rectified sine-wave loading with a $\tau_{z \theta_{\max }}=34.6 \mathrm{kPa}$ is required for $\alpha_{\sigma c}=30^{\circ}$ and $60^{\circ}$ tests, and a $\tau_{z \theta_{\max }}$ of $40 \mathrm{kPa}$ is required for the test at $\alpha_{\sigma c}=45^{\circ}$. 


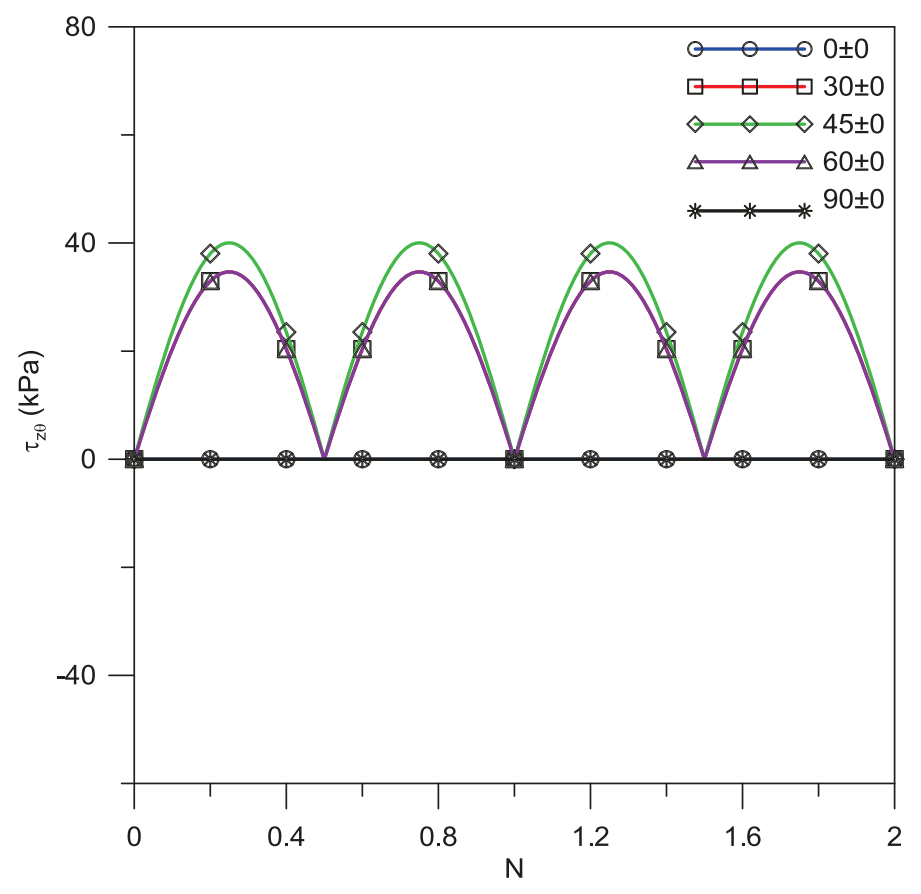

Fig 5.32 Shear stress on the horizontal plan during no rotation for different $\boldsymbol{\alpha}_{\boldsymbol{\sigma} \boldsymbol{c}}$

None of the specimens liquefied when the tests were terminated, and comparisons are made at the end of 60 load cycles in this section. Fig. 5.33 shows the induced excess pore pressure ration during cyclic loading. The least amount of excess pore pressure $\left(20 \%\right.$ of $\sigma_{m c}^{\prime}$ after 60 cycles) is generated when the major principal stress direction was along the deposition direction $\left(\alpha_{\sigma c}=0\right)$. Interestingly, the next lowest pore pressure generation $\left(26 \%\right.$ of $\sigma_{m c}^{\prime}$ after 60 cycles) corresponds to the test where the major principal stress direction was along the bedding planes $\left(\alpha_{\sigma c}=90\right)$. A common feature for both of tests is that no shear stress is applied on the horizontal (bedding) planes during the test

Even though the CSR and the maximum torsinal shear stress on the horizontal plane are identical in the $\alpha_{\sigma c}=30^{\circ}$, and $60^{\circ}$ tests, the excess pore pressure generation in the $\alpha_{\sigma c}=60^{\circ}$ test is twice that of the $\alpha_{\sigma c}=30^{\circ}$ test (approximately 23\% vs $47 \%$ ). Clearly, alignment of the major principal stress toward the bedding plane causes higher excess pore water pressure generation. 
This observation also confirms the formation of anisotropy in soil structure with weaker plane along the bedding plane.

The test at $\alpha_{\sigma c}=45^{\circ}$ shows somewhat lower rate of excess pore pressure development compared to the test at $\alpha_{\sigma c}=60^{\circ}$. The maximum torsional shear stress on the horizontal plane is slightly higher in the $\alpha_{\sigma c}=45^{\circ}$ test, but as noted earlier tests at $\alpha_{\sigma c}=45^{\circ}$ were conducted at intermediate principal stress parameter $b_{\sigma}=0$ (representing axysymmetric conditions) and all other tests were conducted at $b_{\sigma}=0.4$ (representing plane strain conditions). Both, the higher $b_{\sigma}$ value and more closer orientation along the bedding plane would have contributed to the relatively weaker response in the $\alpha_{\sigma c}=60^{\circ}$ test. But the differences have been minor, and this suggests that the higher torsional shear stress on the horizontal plane counteracts the effects of higher $\mathrm{b}$ and $\alpha_{\sigma c}$ somewhat.

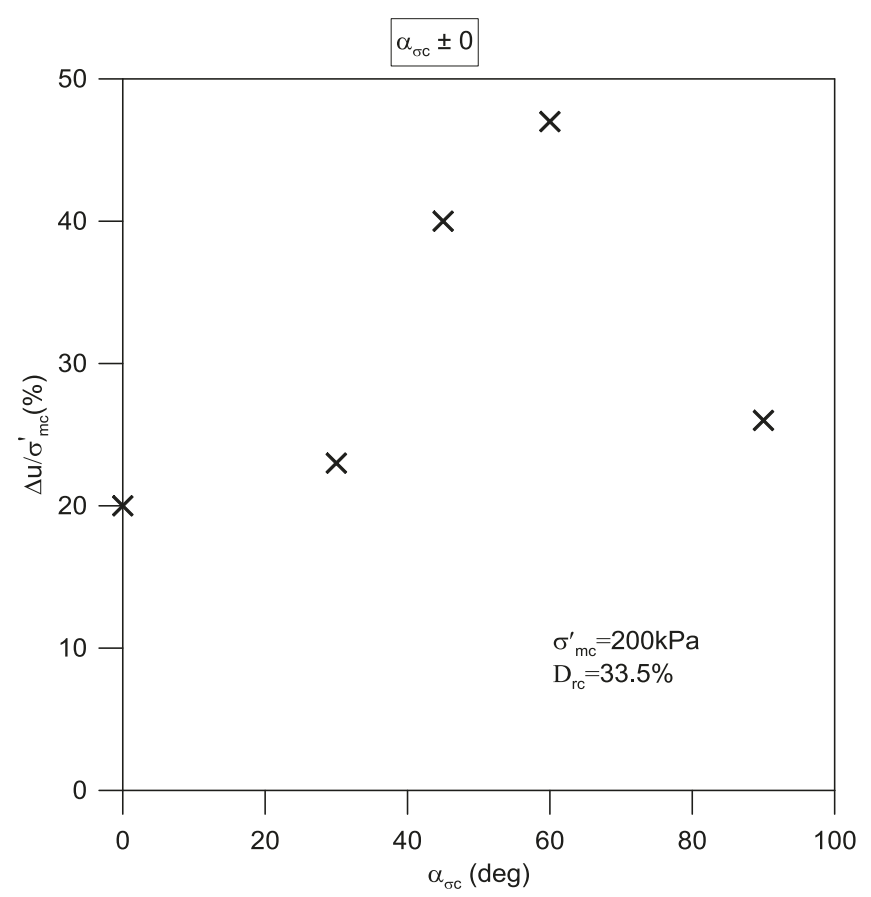

Fig. 5.33 Comparison of excess pore pressure ratio at the end of $60^{\text {th }}$ cycle during $\Delta \alpha_{\sigma}=0^{\circ}$ 


\subsubsection{Behaviour for $\Delta \alpha_{\sigma}=30^{\circ}$}

Fig. 5.34 shows the variation of torsional shear stress $\left(\tau_{z \theta}\right)$ applied on the horizontal plane of the specimen during the application of cyclic loading with fixed rotation of $\Delta \alpha_{\sigma}=30^{\circ}$ but about initial principal stress inclinations of $\alpha_{\sigma c}=0^{\circ}, 30^{\circ}, 45^{\circ}, 60^{\circ}$ and $90^{\circ}$ for cyclic loading at $\mathrm{CSR}=0.2$.

Both $\alpha_{\sigma c}=0^{\circ}$ and $90^{\circ}$ tests require similar shear stress history on the horizontal plane, but in opposite directions. The torsional shear stress applied on the horizontal plane has the same peak value of $34.6 \mathrm{kPa}$ but during $\alpha_{\sigma c}=0^{\circ}$ first half cycle is in compression mode and other half cycle is in extension mode whereas during $\alpha_{\sigma c}=90^{\circ}$ it is vice versa. Hence test with $\alpha_{\sigma c}=0^{\circ}$ and $90^{\circ}$ liquefy in 14 and 9 cycles respectively (Fig. 5.35). This response can clearly be attributed to the inclination of the major principal stress direction along the weaker bedding plane in the $\alpha_{\sigma c}=90^{\circ}$ test.

Even though $\alpha_{\sigma c}=30^{\circ}$ and $60^{\circ}$ tests subjected to $\Delta \alpha_{\sigma}=30^{\circ}$ require the same $\tau_{z} \theta_{\max }=$ $34.6^{\circ}$ their trend of variation is different from that of $\alpha_{\sigma c}=0^{\circ}$ and $90^{\circ}$. The $\alpha_{\sigma c}=0^{\circ}$ and $90^{\circ}$ tests show same maximum shear stress during both half cycles of the cycle where as $\alpha_{\sigma c}=30^{\circ}$ and $60^{\circ}$ tests show $34.6 \mathrm{kPa}$ maximum shear stress during one half cycle and $10 \mathrm{kPa}$ during the other half cycle. Sand liquefied in 19 and 12 cycles respectively (Fig. 5.35) for these tests and the lower number of cycles to liquefaction in the $\alpha_{\sigma c}=60^{\circ}$ test is expected given the orientation of the initial principal stress directions.

The $\tau_{z \theta_{\max }}$ for the test with $\alpha_{\sigma c}=45^{\circ}$ is equal to $21.3 \mathrm{kPa}$. This cause the strongest behaviour in addition to the adverse effect of $b_{\sigma c}=0$ and liquefy the specimen in 18 cycles (Fig. 5.35). 


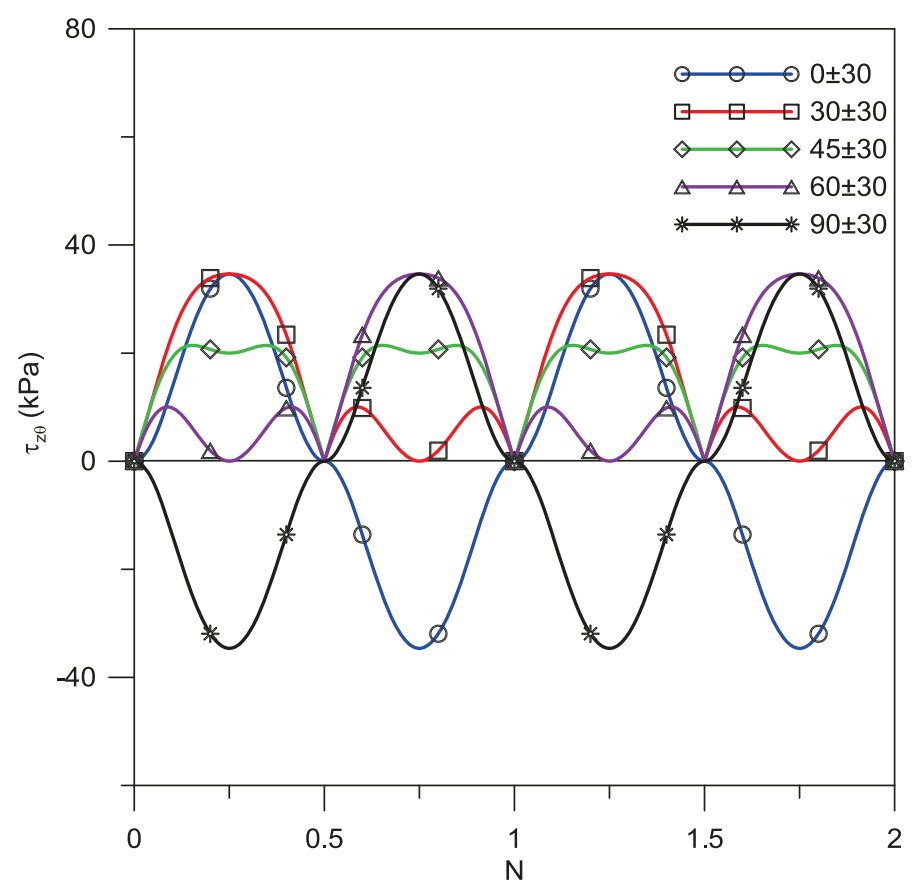

Fig 5.34 Shear stress on the horizontal plan during $\Delta \alpha_{\sigma}=30^{\circ}$ rotation for different $\alpha_{\sigma c}$

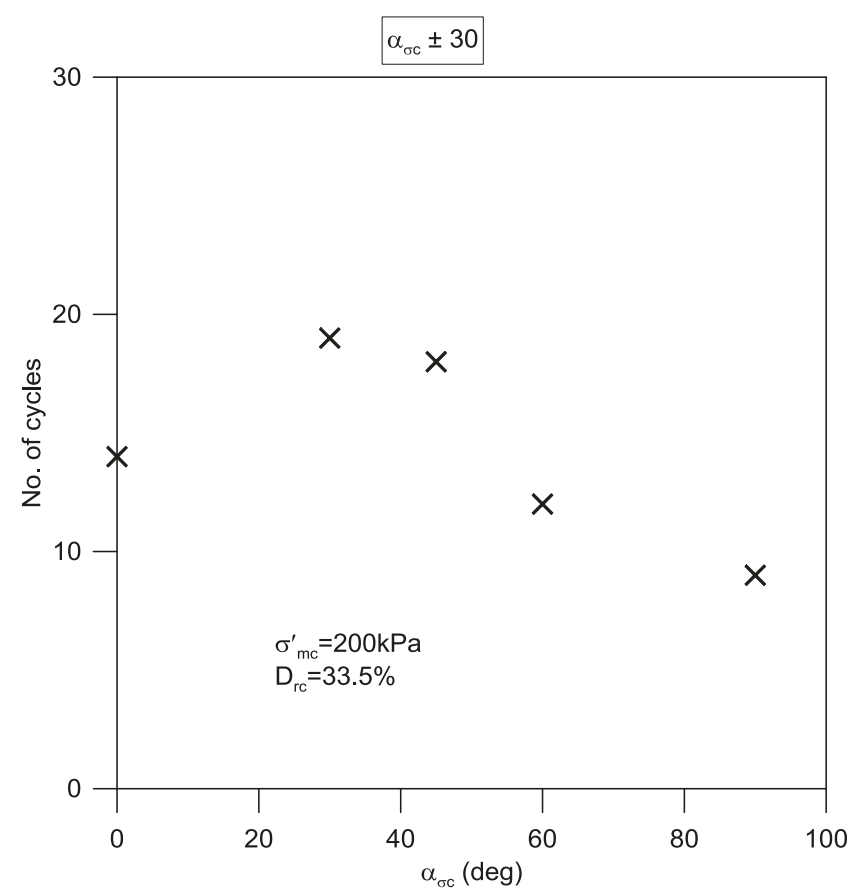

Fig. 5.35 Comparison of number of cycle required for liquefaction during $\Delta \alpha_{\sigma}=30^{\circ}$ 


\subsubsection{Behaviour for $\Delta \alpha_{\sigma}=45^{\circ}$}

Fig. 5.36 shows the variation of torsional shear stress $\left(\tau_{z \theta}\right)$ applied on the horizontal plane of the specimen during the application of cyclic loading with fixed rotation of $\Delta \alpha_{\sigma}=45^{\circ}$ but about initial principal stress inclinations of $\alpha_{\sigma c}=0^{\circ}, 30^{\circ}, 45^{\circ}, 60^{\circ}$ and $90^{\circ}$ for cyclic loading at $\mathrm{CSR}=0.2$. Stress rotation about the $45^{\circ}$ inclination is a special case as it involves repeated sequential activation of the weaker and stronger modes of response during cyclic shearing.

Both $\alpha_{\sigma c}=0^{\circ}$ and $90^{\circ}$ tests require similar shear stress history on the horizontal plane $\left(\tau_{z \theta_{\max }}=40 \mathrm{kPa}\right)$, but with a phase shift of $180^{\circ}$. Thus the loading history is fairly similar and the number of cycles to liquefaction reflects that ( 8 vs 7 cycles). The marginally weaker response in the $\alpha_{\sigma c}=90^{\circ}$ test is because the loading starts along the weaker direction in the first half cycle (subsequent loading mirrors that of $\alpha_{\sigma c}=0^{\circ}$ test). Both $\alpha_{\sigma c}=30^{\circ}$ and $60^{\circ}$ tests require the same $\tau_{z \theta_{\max }}=23.5 \mathrm{kPa}$, but with different variation within the cycle. The differences in cyclic strength has been fairly minor as the sand liquefied in 7 and 9 cycles respectively (Fig. 5.37). The test at $\alpha_{\sigma c}=45^{\circ}$ shows relatively higher resistance (16 cycles to liquefaction) compared to all other tests, but as noted previously the stronger behaviour is mainly due to the axisysmmetric stress conditions compared to the plane strain conditions in the other tests in addition to the relatively smaller $\tau_{z \theta_{\max }}(14.1 \mathrm{kPa})$. 


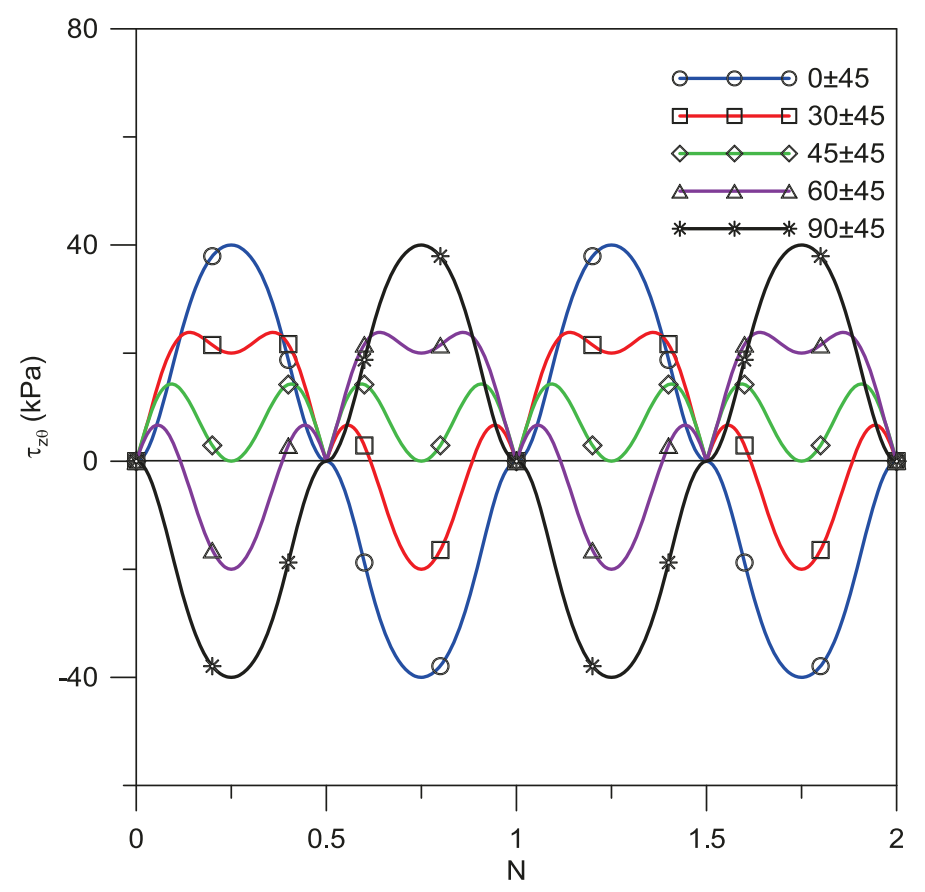

Fig 5.36 Shear stress on the horizontal plan during $\Delta \alpha_{\sigma}=45^{\circ}$ rotation for different $\alpha_{\sigma c}$

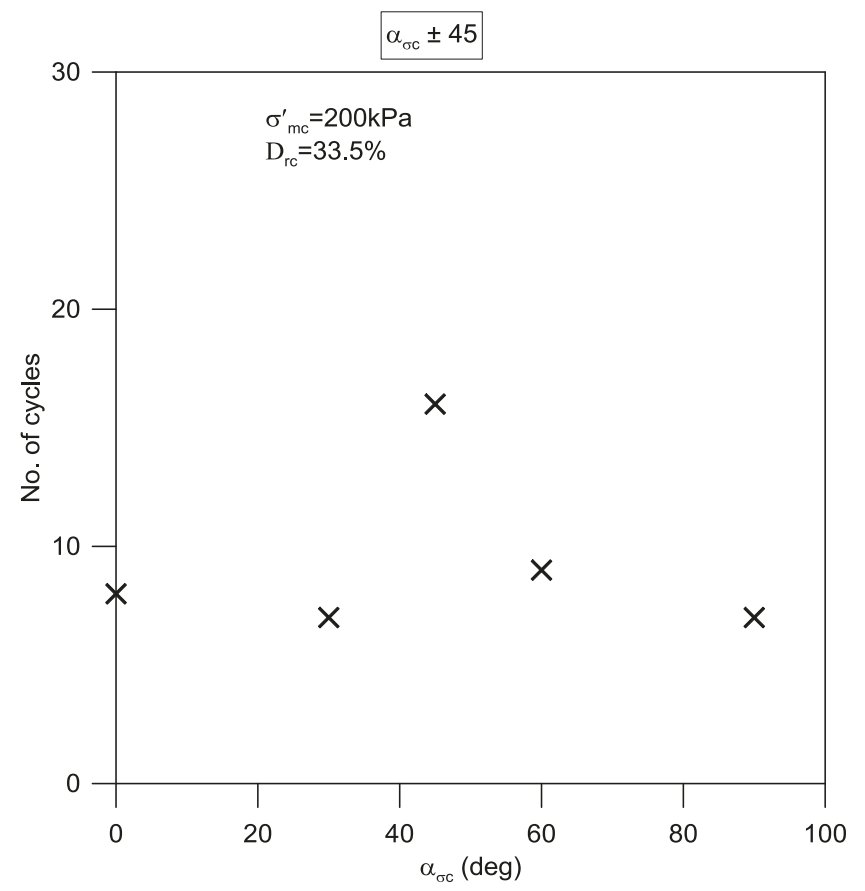

Fig. 5.37 Comparison of number of cycle required for liquefaction during $\Delta \alpha_{\sigma}=45^{\circ}$ 


\subsubsection{Behaviour for $\Delta \alpha_{\sigma}=60^{\circ}$}

Fig. 5.38 shows the variation of torsional shear stress $\left(\tau_{z \theta}\right)$ applied on the horizontal plane of the specimen during the application of cyclic loading with fixed rotation of $\Delta \alpha_{\sigma}=$ $60^{\circ}$ but about initial principal stress inclinations of of $\alpha_{\sigma c}=0^{\circ}, 30^{\circ}, 45^{\circ}, 60^{\circ}$ and $90^{\circ}$ for cyclic loading at $\mathrm{CSR}=0.2$.

As in the $\Delta \alpha_{\sigma}=30^{\circ}$ test discussed earlier, both $\alpha_{\sigma c}=0^{\circ}$ and $90^{\circ}$ tests require similar shear stress history on the horizontal plane, but in opposite directions. A $\tau_{z \theta_{\max }}=34.7 \mathrm{kPa}$ was applied in the $\alpha_{\sigma c}=0^{\circ}$ test in compression mode first half cycle and in extension mode during the other half, whereas in the $\alpha_{\sigma c}=90^{\circ}$ test the first half cycle is in extension mode and the second half in compression mode. Thus the cyclic resistance is fairly similar, and the sand at $\alpha_{\sigma c}$ $=0^{\circ}$ and $90^{\circ}$ liquefied in 10 and 9 cycles respectively (Fig. 5.39).

Even though the tests at $\alpha_{\sigma c}=30^{\circ}$ and $60^{\circ}$ require the same $\tau_{z \theta_{\max }}=34.7 \mathrm{kPa}$ on the horizontal plane, their variation within the cycle is different from that of $\alpha_{\sigma c}=0^{\circ}$ and $90^{\circ}$. The $\alpha_{\sigma c}=0^{\circ}$ and $90^{\circ}$ tests show same maximum shear stress during both half cycles whereas $\alpha_{\sigma c}=$ $30^{\circ}$ and $60^{\circ}$ tests show $34.6 \mathrm{kPa}$ maximum shear stress during one half cycle and $17.3 \mathrm{kPa}$ during the other half cycle. Since the maximum shear stress of $34.6 \mathrm{kPa}$ (on the weakest plane) is encountered only once during each cycle in these tests (as opposed to twice in the $\alpha_{\sigma c}=0^{\circ}$ and $90^{\circ}$ tests) higher cyclic resistance is expected, and the results showing liquefaction in 12 cycles (Fig. 5.39) match the expected response. As noted earlier, two factors (1) relatively smaller $\tau_{z \theta_{\max }}(20 \mathrm{kPa})$ and (2) adverse effect of $b_{c}=0$ stress condition contribute to higher cyclic resistance for the test with $\alpha_{\sigma c}=45^{\circ}$ which liquefied in 19 cycles (Fig. 5.39). 


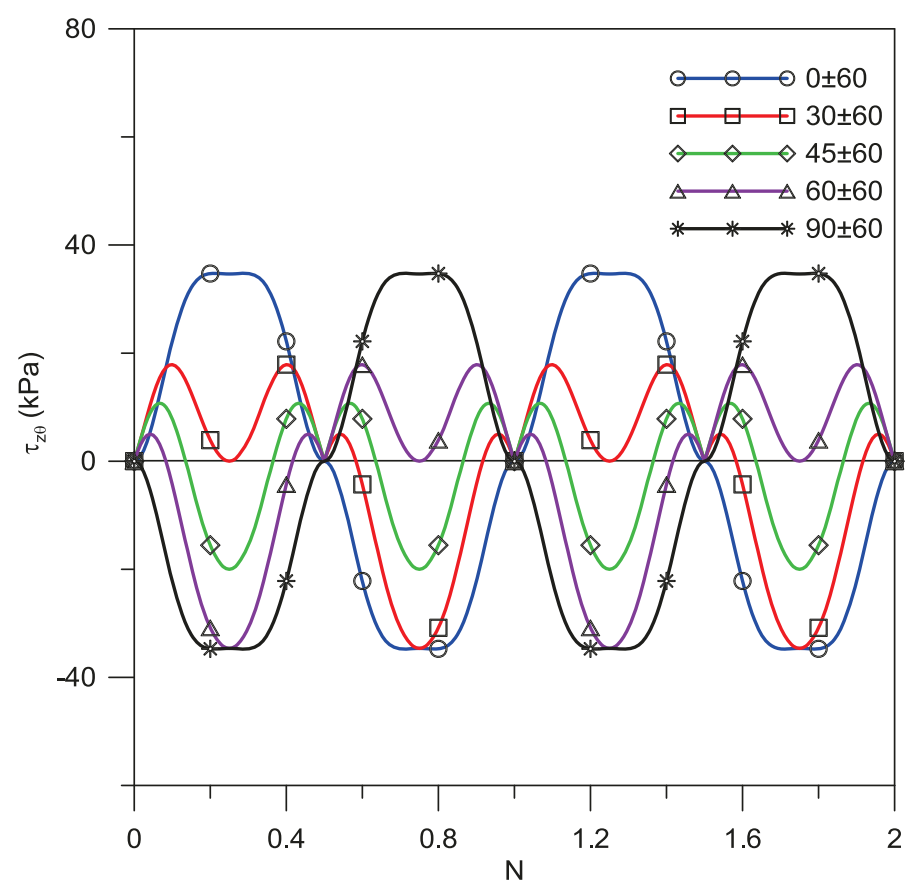

Fig 5.38 Shear stress on the horizontal plan during $\Delta \alpha_{\sigma}=60^{\circ}$ rotation for different $\alpha_{\sigma c}$

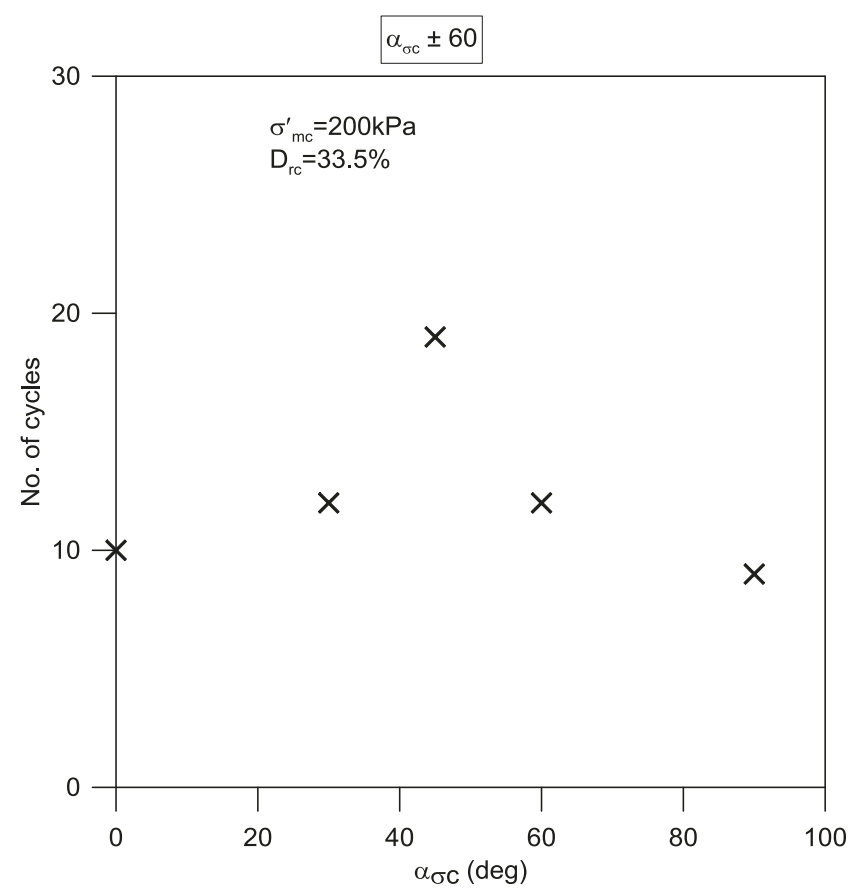

Fig. 5.39 Comparison of number of cycle required for liquefaction during $\Delta \alpha_{\sigma}=60^{\circ}$ 


\subsubsection{Behaviour for $\Delta \alpha_{\sigma}=90^{\circ}$}

Fig. 5.40 shows the variation of torsional shear stress $\left(\tau_{z \theta}\right)$ applied on the horizontal plan of the specimen during the application of cyclic loading with fixed rotation of $\Delta \alpha_{\sigma}=$ $90^{\circ}$ but about initial principal stress inclinations of $\alpha_{\sigma c}=0^{\circ}, 30^{\circ}, 45^{\circ}, 60^{\circ}$ and $90^{\circ}$ for cyclic loading at $\mathrm{CSR}=0.2$.

The torsional shear stress applied on the horizontal plane has the same peak value of 22.5 $\mathrm{kPa}$ but during $\alpha_{\sigma c}=0^{\circ}$ first half cycle is in compression mode and other half cycle is in extension mode whereas during $\alpha_{\sigma c}=90^{\circ}$ it is vice versa. The peak cyclic stress in the $\alpha_{\sigma c}=$ $90^{\circ}$ test is applied when the major principal stress is vertical, and in the $\alpha_{\sigma c}=0^{\circ}$ test when the major principal stress is horizontal. The test with $\alpha_{\sigma c}=0^{\circ}$ and $90^{\circ}$ require 15 and 27 cycles respectively to liquefy (Fig. 5.41). This difference can be attributed to the the lower strength of the sand along the bedding plane which is occurs $\alpha_{\sigma c}=0^{\circ}$ test and higher strength of the sand along the deposition direction which is occurs $\alpha_{\sigma c}=90^{\circ}$ test (given the stress rotation amplitude of $90^{\circ}$ ). The $\alpha_{\sigma c}=30^{\circ}$ and $60^{\circ}$ tests show a maximum torsional shear stress equal to $35.3 \mathrm{kPa}$, and liquefy in 18 cycles (Fig. 5.41), but because of the effect of the direction of the major principal stress noted earlier the $\alpha_{\sigma c}=0^{\circ}$ shows the weakest response in this series.

\subsection{SUMMARY}

The findings of the study carried out to investigate the monotonic and cyclic behaviour of Fraser River sand under smooth principal stress rotation using HCT device are presented in this chapter. The series of experiments were aimed to better understand the influence of initial direction of principal stress, and the influence of the level of rotation on the liquefaction resistance. The results of the series of experiments yield the following conclusions. 


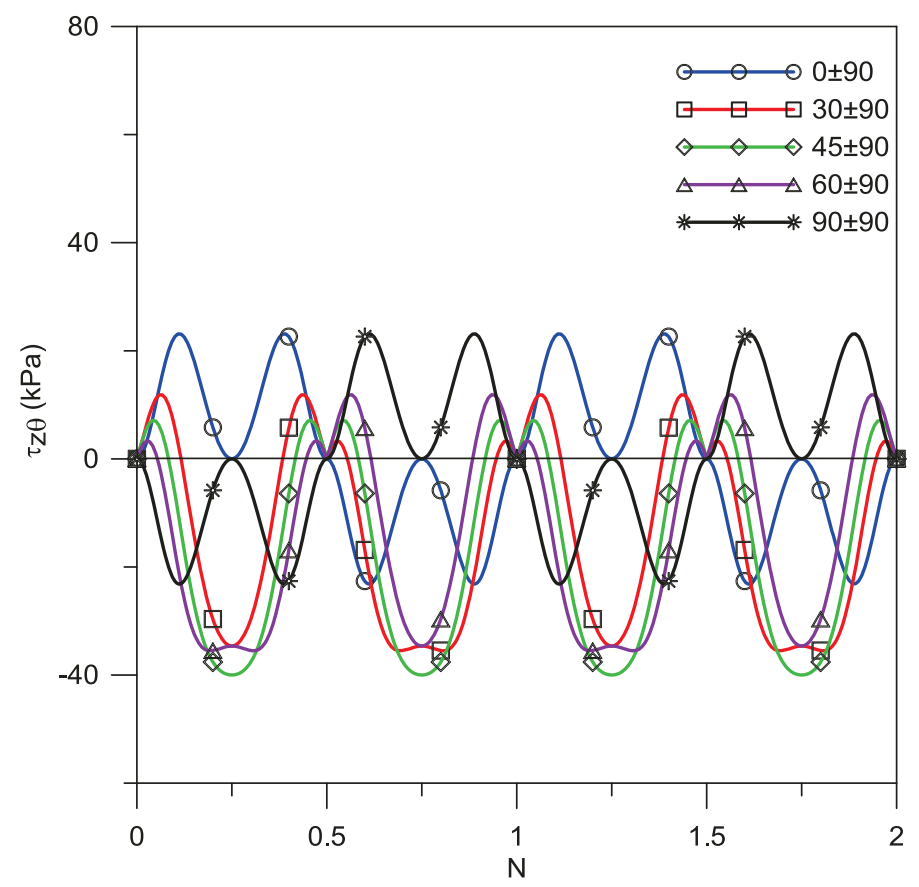

Fig 5.40 Shear stress on the horizontal plan during $\Delta \alpha_{\sigma}=90^{\circ}$ rotation for different $\alpha_{\sigma c}$

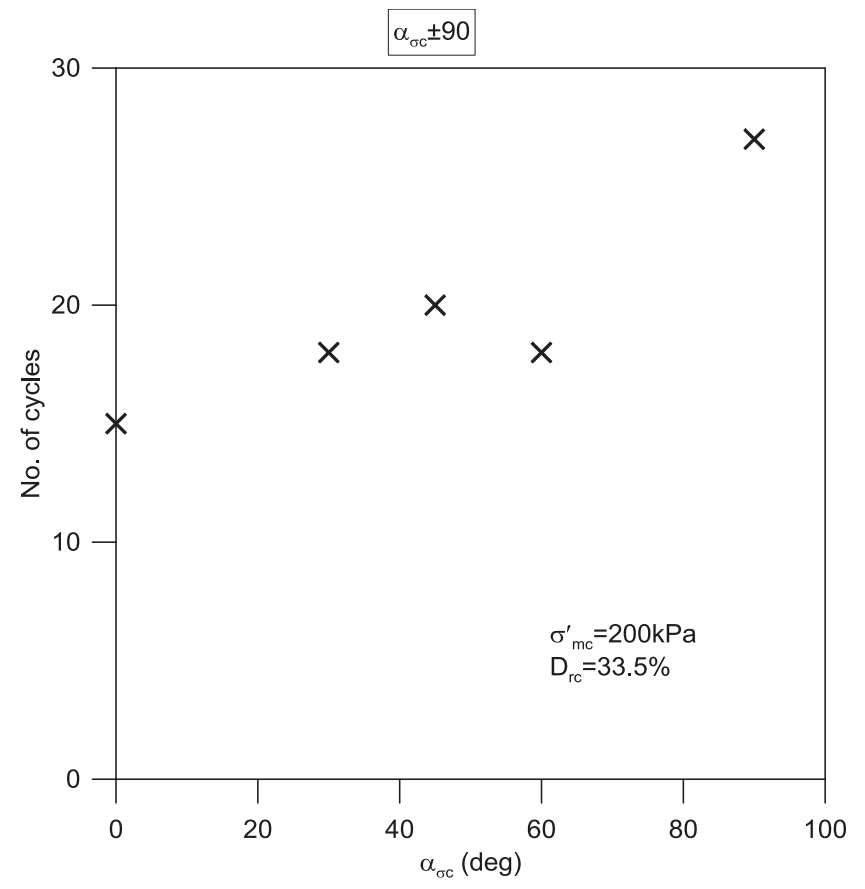

Fig. 5.41 Comparison of number of cycle required for liquefaction during $\Delta \alpha_{\sigma}=90^{\circ}$ 
The test carried out to study the influence of level of rotation on cyclic liquefaction resistance of Fraser River sand specimen clearly indicates that irrespective of the initial principal stress direction, the lowest cyclic resistance corresponds to $45^{\circ}$ rotation of principal stress about its initial position. This finding appears to be consistent with the finding in the literature for initial principal stress direction oriented along the vertical deposition direction (Logeswaran, 2010 and Manmatharajan, 2011). This observation may be explained by the concept of "induced anisotropy" which results during the shearing of the specimen.

Only during $45^{\circ}$ rotation of principal stress both the major principal stress and the minor principal stress will align along a particular direction irrespective of the initial direction of the principal stress. According to the induced anisotropy concept proposed by Oda et al. (1985), in non-spherical particles the direction of contact normals tend to align with the direction of major principal stress and the longitudinal axis of the particles tends to align along the minor principal stress direction. Since for the $45^{\circ}$ rotation of principal stress case in a particular direction major and minor principal stress is acted alternately at their maximum rotation during a cycle. This causes fabric changes during shearing (induced anisotropy) in sand specimen and change the physical configuration of the soil structure alternately. This could potentially weaken the soil specimen and cause liquefaction in less number of cycles.

As indicated in the literature direction of major principal stress with reference to the weakest horizontal plane plays an important role in determining the cyclic liquefaction resistance of sands. However, when principal stress directions continually change, the orientation of the major principal stress at the instant of peak cyclic stress seems to be a critical parameter in controlling the cyclic strength. Fraser River sand exhibits higher cyclic resistance when the nature of the rotation of principal stress causes $\sigma_{1}$ along the deposition direction $\left(\alpha_{\sigma c}=\right.$ 
$90^{\circ}, \Delta \alpha=90^{\circ}$ test) whereas the cyclic resistance reduces drastically when the rotation aligns $\sigma_{1}$ along the bedding plane $\left(\alpha_{\sigma c}=0^{\circ}, \Delta \alpha=90^{\circ}\right.$ test $)$. Further the magnitude of the torsional shear applied on the horizontal plane and the nature of the time history (duration of that near maximum $\tau_{z \theta}$ ) also affects the cyclic resistance of the specimen. Finally the manner of rotation (with or without stress reversals) also influences the behaviour of sand as seen in the tests with no principal stress rotation $\left(\Delta \alpha=0^{\circ}\right)$ or those with $90^{\circ}$ rotation.

The test carried out to study the influence of initial direction of principal stress on cyclic liquefaction resistance of Fraser River sand specimen clearly indicates that the cyclic resistance of sand depends on the magnitude of $\tau_{z \theta_{\max }}$ and the trend of the variation of torsional shear stress on the horizontal plane. Reason for the behaviour compared over the same $\tau_{z \theta_{\max }}$ may be explained by the differences in the direction of major principal stress with reference to the weakest horizontal plane. In some special scenarios, for example the behaviour of $\alpha_{\sigma c}=0^{\circ}$ and $90^{\circ}$ with rotation of $90^{\circ}$, the actual nature of principal stress rotation also influences the behaviour of cyclic liquefaction resistance. 


\section{PORE PRESSURE GENERATION UNDER GENERALIZED LOADING}

\subsection{INTRODUCTION}

Pore pressure generation during undrained loading plays an important role in geotechnical engineering since it is responsible for the reduction in the effective stress, and thus directly affects the undrained strength of soils. The amount of pore pressure induced due to a given change in total stress has generally been divided into two components: firstly a component due to the overall total stress increment (represented by $\Delta \sigma_{3}$ or $\Delta \sigma_{m}$ ), and secondly a component that represents the shear (represented by $\Delta \sigma_{d}$ or $\Delta \tau_{o c t}$ ) induced excess pore water pressure. As discussed in chapter 2, different approaches have been used in practice to quantify the excess pore pressure induced due to a change in total stress in terms of principal stress increments. The theoretical formulations (Skempton 1954; Henkel 1960) were based on presumed isotropic behaviour in soils, and experimental validations of these propositions have been limited to simpler loading conditions (e.g., triaxial compression, extension) only. Verification of the validity of these approaches under generalized field loading conditions in transversely isotropic material would be of significant value to the practice. To the best of my knowledge, no attempt has been made to date to evaluate the applicability of these concepts under generalized three dimensional loading conditions. This chapter presents the results of a series of tests conducted to assess whether the formulations can be applicable under three-dimensional loading. 


\subsection{ANALYTICAL FORMULATIONS FOR PORE PRESSURE GENERATION IN AN}

\section{ELASTIC MATERIAL}

As discussed in chapter 2, Skempton (1954) proposed that an isotropic increment of stresses in a saturated soil generates pore pressure equal to the magnitude of the stress increments. This proposition has been well established in the literature for hydrostatic initial states. Experiments reported herein demonstrate that the formulation holds true under generalized loading conditions with an arbitrary value of intermediate principal stress, and the direction of the major principal stress even in soils that do not have an isotropic fabric. Several studies in the literature indicate that water deposited sands have a transversely isotropic (called cross anisotropic by some researchers) fabric, and that the horizontal plane is a plane of isotropy.

The relationship between principal stresses and strains for an elastic cross- anisotropic material is generally expressed as $\{\Delta \epsilon\}=[C]\{\Delta \sigma\}$ where $[C]$ is the compliance matrix. In general form this equation is

$$
\left[\begin{array}{l}
\Delta \varepsilon_{11} \\
\Delta \varepsilon_{22} \\
\Delta \varepsilon_{33}
\end{array}\right]=\left[\begin{array}{lll}
c_{11} & c_{12} & c_{12} \\
c_{12} & c_{33} & c_{13} \\
c_{12} & c_{13} & c_{33}
\end{array}\right]\left[\begin{array}{l}
\Delta \sigma_{11}^{\prime} \\
\Delta \sigma_{22}^{\prime} \\
\Delta \sigma_{33}^{\prime}
\end{array}\right]
$$

\subsubsection{Hydrostatic stress increment in a transversely isotropic soil}

The vertical axis is an axis of symmetry in most cross-anisotropic (transversely isotropic) soils, and considering an isotropic stress increment the equation can be written using commonly understood parameters as 


$$
\left\{\begin{array}{c}
\Delta \epsilon v \\
\Delta \epsilon_{h} \\
\Delta \epsilon_{h}
\end{array}\right\}=\left[\begin{array}{ccc}
\frac{1}{E_{v}} & \frac{-v_{v h}}{E_{v}} & \frac{-v_{v h}}{E_{v}} \\
\frac{-v_{v h}}{E_{v}} & \frac{1}{E_{h}} & \frac{-v_{h h}}{E_{h}} \\
\frac{-v_{v h}}{E_{v}} & \frac{-v_{h h}}{E_{h}} & \frac{1}{E_{h}}
\end{array}\right]\left\{\begin{array}{c}
\Delta \sigma^{\prime} \\
\Delta \sigma^{\prime} \\
\Delta \sigma^{\prime}
\end{array}\right\}
$$

Thus

$$
\left(\Delta \epsilon_{v}+2 \Delta \epsilon_{h}\right)=\left[\frac{\left(1-4 v_{v h}\right)}{E_{v}}+\frac{2\left(1-v_{h h}\right)}{E_{h}}\right] \Delta \sigma^{\prime}
$$

In other words,

$$
\left(\Delta \epsilon_{v}+2 \Delta \epsilon_{h}\right)=\left[\frac{\left(1-4 v_{v h}\right)}{E_{v}}+\frac{2\left(1-v_{h h}\right)}{E_{h}}\right](\Delta \sigma-\Delta u)
$$

The total volume remains constant when a fully saturated soil is subjected to truly undrained loading. The increase in pore water pressure $\Delta u$ will cause a change in the pore water volume, $\Delta V_{P}$ which is given by $\Delta V_{P} / V_{P}=C_{w} \Delta u$ where $C_{w}$ is the compressibility and $V_{P}$ the volume of the pore fluid respectively. For a hydrostatic total stress increment $\Delta \sigma$ the change in effective stress is also hydrostatic and is given by $\Delta \sigma^{\prime}=\Delta \sigma-\Delta u$. The volumetric strain of the soil skeleton $\Delta \epsilon_{v}=\Delta V / V_{o}=\Delta \epsilon_{1}+\Delta \epsilon_{2}+\Delta \epsilon_{3}$. In a transversely isotropic material with a vertical axis of symmetry, this reduces to $\Delta \epsilon_{v}=\Delta V / V_{0}=\Delta \epsilon_{v}+2 \Delta \epsilon_{h}$. For a soil element with porosity, $n$ the volume change components (pore fluid \& soil skeleton) will be $\Delta V_{P}=$ $C_{w} n V_{0} \Delta u$ and $\Delta V=\left(\Delta \epsilon_{v}+2 \Delta \epsilon_{h}\right) V_{0}$. For a truly undrained test, $\Delta V_{P}=\Delta V$, and thus $\left(\Delta \epsilon_{v}+\right.$ $\left.2 \Delta \epsilon_{h}\right)=C_{w} n \Delta u$. This leads to the generalized equation for pore pressure generation in a transversely isotropic soil under hydrostatic stress increment 


$$
B=\frac{\Delta u}{\Delta \sigma}=\frac{1}{1+\frac{C_{w} n}{\frac{\left(1-4 v_{v h}\right)}{E_{v}}+\frac{2\left(1-v_{h h}\right)}{E_{h}}}}
$$

The term $\frac{\left(1-4 v_{v h}\right)}{E_{v}}+\frac{2\left(1-v_{h h}\right)}{E_{h}}$ is the volumetric compressibility $C_{s k-3 D}$ of the soil skeleton and if the soil is assumed to be isotropic $\left(E=E_{v}=E_{h} \& v_{v h}=v_{h h}=v\right)$ this equation will simplify to the original Skempton's formulation with $C_{s k}$ iso $=3(1-2 v) / E$

$$
B=\frac{\Delta u}{\Delta \sigma}=\frac{1}{1+\frac{n C_{w}}{C_{\text {sk iso }}}}
$$

Test results presented in this chapter demonstrating that the excess pore pressure generation under generalized initial states is equal to the hydrostatic stress increment indicate that compressibility of water $C_{w}$ remains fairly negligible compared to $C_{s k-3 D}$.

\subsubsection{Non-hydrostatic stress increment in a transversely isotropic soil}

When a non-hydrostatic stress increment in applied to the transversely isotropic soil element, the principal strains can be determined from the following equation if principal stress axis coincides with the material's axis of symmetry $\left(\alpha_{\sigma}=0^{\circ}\right.$ or $\left.90^{\circ}\right)$

$$
\left\{\begin{array}{c}
\Delta \epsilon_{1} \\
\Delta \epsilon_{2} \\
\Delta \epsilon_{3}
\end{array}\right\}=\left[\begin{array}{ccc}
\frac{1}{E_{v}} & \frac{-v_{v h}}{E_{v}} & \frac{-v_{v h}}{E_{v}} \\
\frac{-v_{v h}}{E_{v}} & \frac{1}{E_{h}} & \frac{-v_{h h}}{E_{h}} \\
\frac{-v_{v h}}{E_{v}} & \frac{-v_{h h}}{E_{h}} & \frac{1}{E_{h}}
\end{array}\right]\left\{\begin{array}{c}
\Delta \sigma_{1}^{\prime} \\
\Delta \sigma_{2}{ }^{\prime} \\
\Delta \sigma_{3}{ }^{\prime}
\end{array}\right\}
$$

Since $\Delta \epsilon_{v}=\Delta \epsilon_{1}+\Delta \epsilon_{2}+\Delta \epsilon_{3}$, expanding the above equation leads to

$$
\Delta \epsilon_{v}=\frac{\left(1-v_{v h}\right) \Delta \sigma_{1}^{\prime}-v_{v h}\left(\Delta \sigma_{1}^{\prime}+\Delta \sigma_{2}^{\prime}+\Delta \sigma_{3}^{\prime}\right)}{E_{v}}+\frac{\left(1-v_{h h}\right)\left(\Delta \sigma_{2}^{\prime}+\Delta \sigma_{3}^{\prime}\right)}{E_{h}}
$$

and 


$$
\Delta u=\frac{1}{C_{w} n}\left[\frac{\left(1-v_{v h}\right) \Delta \sigma_{1}^{\prime}-v_{v h}\left(\Delta \sigma_{1}^{\prime}+\Delta \sigma_{2}^{\prime}+\Delta \sigma_{3}^{\prime}\right)}{E_{v}}+\frac{\left(1-v_{h h}\right)\left(\Delta \sigma_{2}^{\prime}+\Delta \sigma_{3}^{\prime}\right)}{E_{h}}\right]
$$

Substituting $\Delta \sigma_{i}^{\prime}=\Delta \sigma_{i}-u: i=1 . .3$ yields

$$
\begin{gathered}
\Delta u=\frac{1}{C_{w} n}\left[\frac{\left(1-v_{v h}\right) \Delta \sigma_{1}-v_{v h}\left(\Delta \sigma_{1}+\Delta \sigma_{2}+\Delta \sigma_{3}\right)}{E_{v}}+\frac{\left(1-v_{h h}\right)\left(\Delta \sigma_{2}+\Delta \sigma_{3}\right)}{E_{h}}\right. \\
\left.-\frac{\left(1-4 v_{v h}\right) \Delta u}{E_{v}}-\frac{2\left(1-v_{h h}\right) \Delta u}{E_{h}}\right]
\end{gathered}
$$

Rearranging yields an equation in terms of principal stress increments $\Delta \sigma_{1}, \Delta \sigma_{2}$ and $\Delta \sigma_{3}$ as

$$
\Delta u=\frac{1}{C_{w} n} \frac{\left[\frac{\left(1-v_{v h}\right) \Delta \sigma_{1}-v_{v h}\left(\Delta \sigma_{1}+\Delta \sigma_{2}+\Delta \sigma_{3}\right)}{E_{v}}+\frac{\left(1-v_{h h}\right)\left(\Delta \sigma_{2}+\Delta \sigma_{3}\right)}{E_{h}}\right]}{\left(1+\frac{\left(1-4 v_{v h}\right)}{C_{w} n E_{v}}+\frac{2\left(1-v_{h h}\right)}{C_{w} n E_{h}}\right)}
$$

Clearly, excess pore pressure generation for a given stress increment $\left(\Delta \sigma_{1}, \Delta \sigma_{2}, \Delta \sigma_{3}\right)$ will depend on the nature of anisotropy (i.e., relative values of $E_{v}, E_{h}, v_{v h}$ and $v_{h h}$ ) and the magnitude of the stress increments. For an isotropic material, $E=E_{v}=E_{h} \& v_{v h}=v_{h h}=v$ and thus the equation simplifies to

$$
\Delta u=\frac{\left(\Delta \sigma_{1}+\Delta \sigma_{2}+\Delta \sigma_{3}\right)}{3+\frac{n C_{w} E}{(1-2 v)}}
$$

or

$$
\Delta u=\frac{1}{1+\frac{n C_{w}}{C_{\text {sk iso }}}} \cdot \Delta \sigma_{m}
$$

where $\Delta \sigma_{m}=\left(\Delta \sigma_{1}+\Delta \sigma_{2}+\Delta \sigma_{3}\right) / 3$ is the mean normal stress increment, and since $1+n C_{w} / C_{\text {sk iso }} \approx 1$ for most saturated natural soils, the excess pore pressure generated will be 
equal to the mean normal stress increment for purely elastic materials. For simple 1-D loading, the Skempton's A value would thus be $1 / 3$ as widely recognized in the literature. Such simplified formulation will not be possible under generalized three dimensional loading of an elasto-plastic material and the formulation will have to include a complete $6 \times 6$ compliance matrix.

An alternate formulating, following Skempton's hypothesis can be attempted by considering the stress increment vector as the sum of the spherical and deviatoric stress vectors,

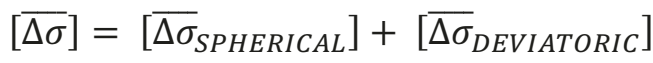

$$
\begin{aligned}
& \left\{\begin{array}{c}
\Delta \sigma_{1} \\
\Delta \sigma_{2} \\
\Delta \sigma_{3}
\end{array}\right\}=\left\{\begin{array}{c}
\Delta \sigma_{3} \\
\Delta \sigma_{3} \\
\Delta \sigma_{3}
\end{array}\right\}+\left\{\begin{array}{c}
\Delta \sigma_{1}-\Delta \sigma_{3} \\
\Delta \sigma_{2}-\Delta \sigma_{3} \\
0
\end{array}\right\}
\end{aligned}
$$

Using this formulation, equation 6.11 would take the form

$$
\Delta u=\frac{\Delta \sigma_{3}}{1+\frac{C_{w} n}{\frac{\left(1-4 v_{v h}\right)}{E_{v}}+\frac{2\left(1-v_{h h}\right)}{E_{h}}}}+\frac{2}{C_{w} n} \frac{\left[\frac{\left(1-2 v_{v h}\right) \Delta \tau_{13}-v_{v h} \Delta \tau_{23}}{E_{v}}+\frac{\left(1-v_{h h}\right) \Delta \tau_{23}}{E_{h}}\right]}{\left(1+\frac{\left(1-4 v_{v h}\right)}{C_{w} n E_{v}}+\frac{2\left(1-v_{h h}\right)}{C_{w} n E_{h}}\right)}
$$

where $\Delta \tau_{13}=\left(\Delta \sigma_{1}-\Delta \sigma_{3}\right) / 2$ and $\Delta \tau_{23}=\left(\Delta \sigma_{2}-\Delta \sigma_{3}\right) / 2$ are the maximum shear stresses in the major-minor and intermediate-minor planes respectively. For a triaxial compression test, $\Delta \tau_{23}=0$ and equation 6.16 can be simplified as

$$
\Delta u=B\left[\Delta \sigma_{3}+A \Delta \sigma_{d}\right]
$$

where

$$
B=1 / 1+\frac{C_{w} n}{\frac{\left(1-4 v_{v h}\right)}{E_{v}}+\frac{2\left(1-v_{h h}\right)}{E_{h}}}
$$


and

$$
A=\frac{1}{B} \cdot \frac{\left(1-2 v_{v h}\right)}{\left(E_{v} C_{w} n+\left(1-4 v_{v h}\right)+2\left(1-v_{h h}\right) \frac{E_{v}}{E_{h}}\right)}
$$

For a triaxial extension test, $\Delta \tau_{23}=\Delta \tau_{13}$ and thus the corresponding Skempton's $A$ parameter will be

$$
A=\frac{1}{B} \cdot \frac{\left(1-3 v_{v h}\right)+\left(1-v_{h h}\right) \frac{E_{v}}{E_{h}}}{\left(E_{v} C_{w} n+\left(1-4 v_{v h}\right)+2\left(1-v_{h h}\right) \frac{E_{v}}{E_{h}}\right)}
$$

Equations 6.19 and 6.20 indicate that Skempton's $A$ value will not be equal under compression and extension loading, even if the transversely isotropic material is purely elastic. For an isotropic material, these formulations will simplify to equation 1.21 as formulated by Skempton.

$$
\begin{gathered}
B=\frac{1}{1+\frac{n C_{w}}{C_{\text {Sk iso }}}} \\
A_{T C}=\frac{1}{3} \quad \& \quad A_{T E}=\frac{2}{3}
\end{gathered}
$$

These elastic formulations indicate that pore pressure parameters depend only on the increments, and not on the initial effective stress states. It would be of interest to evaluate whether this premise holds for natural soils, which in addition to being non-isotropic, possess plastic deformation characteristics. 


\subsection{Test Program}

Tests intended to explore the effect of initial effective stress ratio (i.e. static shear) and the direction of principal stresses under plane strain loading conditions (as opposed to the axisymmetric conditions that prevail in a triaxial test) on pore water pressure generation were conducted using the HCT device. Two series of tests were conducted in this component of the research. In the first series, different hydrostatic stress increments $(\Delta \sigma=50,100$ and $150 \mathrm{kPa}$ or $\Delta \sigma=50,75$, and $100 \mathrm{kPa}$ ) were applied to specimens at generalized, non-hydrostatic initial states. These tests enable the determination of the relationship between the amount of excess pore pressure generated and the hydrostatic stress increment under a generalized initial stress state. Specimens were consolidated to a fixed value of intermediate principal stress parameter $\left(b_{\sigma c}=0.4\right)$, and different values of effective mean normal stress $\left(\sigma_{m c}^{\prime}=50\right.$ or $\left.100 \mathrm{kPa}\right)$, inclination of major principal stress $\left(\alpha_{\sigma c}=0^{\circ}\right.$ or $\left.45^{\circ}\right)$ and $k_{c}$ ranging from 1.0 to 3.0. The data acquisition and control program applied the increments simultaneously such that there is no change in the deviatoric stress applied to the specimen; $\sigma_{d}=\left(k_{c}-1\right) \times \sigma_{3 c}^{\prime}$ remained a constant throughout the loading sequence, and $\Delta \sigma_{m}=\Delta \sigma_{3}=\Delta \sigma$. The state of saturation in the specimens was confirmed initially at a hydrostatic stress state, and the Skempton's $B$ value was 0.99 or better was ensured in all tests. The details of the tests conducted in this series, together with the measured pore pressure increment are given in Table 6.1. 
Table 6.1 Initial and loading state of tests conducted under different total stress path for Skempton's " $B$ " value test

\begin{tabular}{|c|c|c|c|c|c|c|c|c|}
\hline \multirow{2}{*}{ Test No } & \multicolumn{5}{|c|}{ Consolidation state } & \multicolumn{3}{|c|}{ Loading state } \\
\hline & $e_{c}$ & $\sigma_{m c}^{\prime}(\mathrm{kPa})$ & $\mathrm{k}_{\mathrm{c}}$ & $b_{c}$ & $\alpha_{\sigma c}($ Deg) & $\Delta \sigma(\mathrm{kPa})$ & $\Delta \mathrm{u}(\mathrm{kPa})$ & $\Delta \mathrm{u} / \Delta \sigma$ \\
\hline $\begin{array}{l}\text { "B" } \\
\text { Value }\end{array}$ & 0.777 & 25 & 1.0 & - & - & 25 & 24.85 & 0.994 \\
\hline \multirow{3}{*}{ PP01A } & \multirow{3}{*}{0.774} & \multirow{3}{*}{50} & \multirow{3}{*}{1.5} & \multirow{3}{*}{0.4} & \multirow{3}{*}{0} & 50 & 49.3 & 0.986 \\
\hline & & & & & & 100 & 98.91 & 0.989 \\
\hline & & & & & & 150 & 148.98 & 0.993 \\
\hline \multirow{3}{*}{ PP01B } & \multirow{3}{*}{0.773} & \multirow{3}{*}{50} & \multirow{3}{*}{2.0} & \multirow{3}{*}{0.4} & \multirow{3}{*}{0} & 50 & 49.01 & 0.980 \\
\hline & & & & & & 100 & 98.52 & 0.985 \\
\hline & & & & & & 150 & 148.52 & 0.990 \\
\hline \multirow{3}{*}{ PP01C } & \multirow{3}{*}{0.772} & \multirow{3}{*}{50} & \multirow{3}{*}{3.0} & \multirow{3}{*}{0.4} & \multirow{3}{*}{0} & 50 & 49.06 & 0.981 \\
\hline & & & & & & 100 & 99.05 & 0.991 \\
\hline & & & & & & 150 & 148.53 & 0.990 \\
\hline $\begin{array}{l}\text { "B" } \\
\text { Value }\end{array}$ & 0.765 & 25 & 1.0 & - & - & 25 & 24.93 & 0.997 \\
\hline \multirow{3}{*}{ PP02A } & \multirow{3}{*}{0.763} & \multirow{3}{*}{100} & \multirow{3}{*}{1.3} & \multirow{3}{*}{0.4} & \multirow{3}{*}{45} & 50 & 49.4 & 0.988 \\
\hline & & & & & & 75 & 74.6 & 0.995 \\
\hline & & & & & & 100 & 100 & 1.000 \\
\hline
\end{tabular}




\begin{tabular}{|c|c|c|c|c|c|c|c|c|}
\hline \multirow{3}{*}{ РP02B } & \multirow{3}{*}{0.762} & \multirow{3}{*}{100} & \multirow{3}{*}{1.5} & \multirow{3}{*}{0.4} & \multirow{3}{*}{45} & 50 & 49.2 & 0.984 \\
\hline & & & & & & 75 & 73.7 & 0.983 \\
\hline & & & & & & 100 & 98.5 & 0.985 \\
\hline \multirow{3}{*}{ PP02C } & \multirow{3}{*}{0.761} & \multirow{3}{*}{100} & \multirow{3}{*}{1.8} & \multirow{3}{*}{0.4} & \multirow{3}{*}{45} & 50 & 49.1 & 0.982 \\
\hline & & & & & & 75 & 73.7 & 0.983 \\
\hline & & & & & & 100 & 99.0 & 0.990 \\
\hline \multirow{3}{*}{ PP02D } & \multirow{3}{*}{0.760} & \multirow{3}{*}{100} & \multirow{3}{*}{2.0} & \multirow{3}{*}{0.4} & \multirow{3}{*}{45} & 50 & 49.3 & 0.986 \\
\hline & & & & & & 75 & 73.6 & 0.981 \\
\hline & & & & & & 100 & 98.8 & 0.988 \\
\hline
\end{tabular}

The second series of tests were intended to quantify shear (or deviatoric) stress induced excess pore pressure generation, and evaluate the Skempton's $A$ and Henkel's $a$ parameters under generalized loading conditions. Fully saturated specimens were consolidated to a fixed value of intermediate principal stress parameter $\left(b_{\sigma c}=0.4\right)$, and effective mean normal stress $\left(\sigma_{m c}^{\prime}=100 \mathrm{kPa}\right)$, and to different inclination of major principal stress $\left(\alpha_{\sigma c}=0^{\circ}, 45^{\circ} \& 90^{\circ}\right)$ and initial effective stress ratio $\left(k_{c}=1.0,1.5 \& 2.0\right)$.

Table 6.2 details the test parameters used in this series of tests. Unlike the previous series of tests where the stress increment was hydrostatic, specimens in this series were subjected to two set of non-hydrostatic stress increment vector given by $\Delta \sigma_{1}=25 \mathrm{kPa}, \Delta \sigma_{2}=17.5 \mathrm{kPa}$, $\Delta \sigma_{3}=12.5 \mathrm{kPa}$ and $\Delta \sigma_{1}=100 \mathrm{kPa}, \Delta \sigma_{2}=70 \mathrm{kPa}, \Delta \sigma_{3}=50 \mathrm{kPa}$. The purpose using two such set of stress increments was to evaluate the capability of the system in measuring shear 
induced excess pore water pressure at small stress increments. Both the direction of the major principal stress $\alpha_{\sigma}$ and the value of the intermediate principal stress parameter $b_{\sigma}$ remained constant throughout the test. A staged testing approach was used to conduct the tests. At the end of each test phase, the specimen was sheared drained to reach the initial $k_{c}$ level which requires higher effective stress states. To maintain consistency in the testing program, this was done by adjusting both intermediate and minor principal stresses while keeping the major principal stress constant to reach the desired stress ratio. During the whole test phase no unloading in major principal stress occurred. The test procedure followed ensured that the material remained normally consolidated in terms of major principal stress during each stage of the loading. However, the void ratio of the specimens systematically changed during the testing as the specimens were consolidated to progressively higher static shear stress bias, but the amount of change was negligible ( $\Delta e=0.002$ or less in most cases) 
Table 6.2 Initial and loading state of tests conducted under different total stress path for Skempton's "A" and Henkel's " $a$ " value test

\begin{tabular}{|c|c|c|c|c|c|c|c|c|c|}
\hline \multirow{2}{*}{ Test No } & \multicolumn{5}{|c|}{ Consolidation state } & \multicolumn{3}{|c|}{ Loading state } & \multirow{2}{*}{$\begin{array}{c}\text { Stress } \\
\text { Increment } \\
(\mathrm{kPa})\end{array}$} \\
\hline & $e_{c}$ & $\sigma_{m c}^{\prime}(\mathrm{kPa})$ & $k_{c}$ & $b_{c}$ & $\alpha_{c}(\operatorname{deg})$ & $k_{\sigma}$ & $b_{\sigma}$ & $\alpha_{\sigma}$ & \\
\hline PP03A & 0.736 & 100 & 1.0 & - & - & 1.0 & - & - & \\
\hline РP03B & 0.744 & 100 & 1.5 & 0.4 & 0 & 1.5 & 0.4 & 0 & $\Delta \sigma_{1}=25$ \\
\hline PP03C & 0.741 & 100 & 2.0 & 0.4 & 0 & 2.0 & 0.4 & 0 & $\Delta \sigma_{2}=17.5$ \\
\hline PP04A & 0.749 & 100 & 1.0 & - & - & 1.0 & - & - & \\
\hline PP04B & 0.747 & 100 & 1.5 & 0.4 & 45 & 1.5 & 0.4 & 45 & $\Delta \sigma_{1}=100$ \\
\hline PP04C & 0.753 & 100 & 2.0 & 0.4 & 45 & 2.0 & 0.4 & 45 & $\Delta \sigma_{2}=70$ \\
\hline PP05A & 0.752 & 100 & 1.0 & - & - & 1.0 & - & - & $\Delta \sigma_{3}=50$ \\
\hline PP05B & 0.759 & 100 & 1.5 & 0.4 & 90 & 1.5 & 0.4 & 90 & \\
\hline PP05C & 0.758 & 100 & 2.0 & 0.4 & 90 & 2.0 & 0.4 & 90 & \\
\hline
\end{tabular}




\subsection{SKEMPTON'S B PARAMETER UNDER GENERALIZED LOADING}

Figures 6.1 to 6.3 show the variation of the generated excess pore water pressure as a function of the hydrostatic stress increment $\Delta \sigma$ for the tests conducted at $\sigma_{m c}^{\prime}=50 \mathrm{kPa}$ and $\alpha_{\sigma}=0^{\circ}$ together with the statistics of the least squares fit. Regardless of the initial $k_{c}$ value, the best-fit line passes through the origin and has a gradient of approximately 1.0, (slope angle varying between $44.65^{\circ}-44.77^{\circ}$ ) which is the essentially Skempton's $B$ value for a fully saturated specimen. The Skempton's $B$ value measured under hydrostatic initial stress state for this specimen was 0.994 and these results clearly demonstrate that the Skempton's $B$ value is not affected by the non-hydrostatic stress state, or the plane strain deformation constraint. Similar data, but at $\sigma_{m c}^{\prime}=100 \mathrm{kPa}$ and principal stress directions inclined at $\alpha_{\sigma}=45^{\circ}$ shown in Figures 6.4 to 6.7 yield similar results $\left(B \sim 1\right.$; slope angle: $\left.44.54^{\circ}-44.91^{\circ}\right)$ and demonstrate that the inclinations of principal stress with respect to the bedding plane has no effect on pore pressure generation under a hydrostatic stress increment.

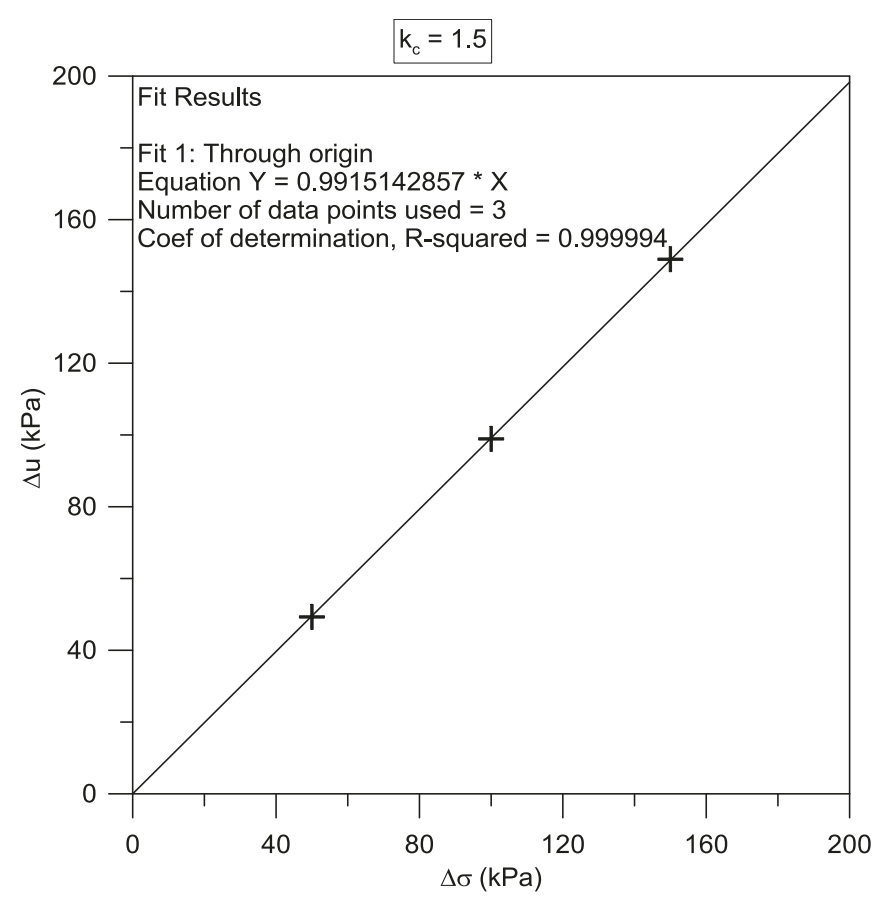

Fig. 6.1 Excess pore pressure induced during isotropic increment of principal stresses for $k_{c}=1.5$ 


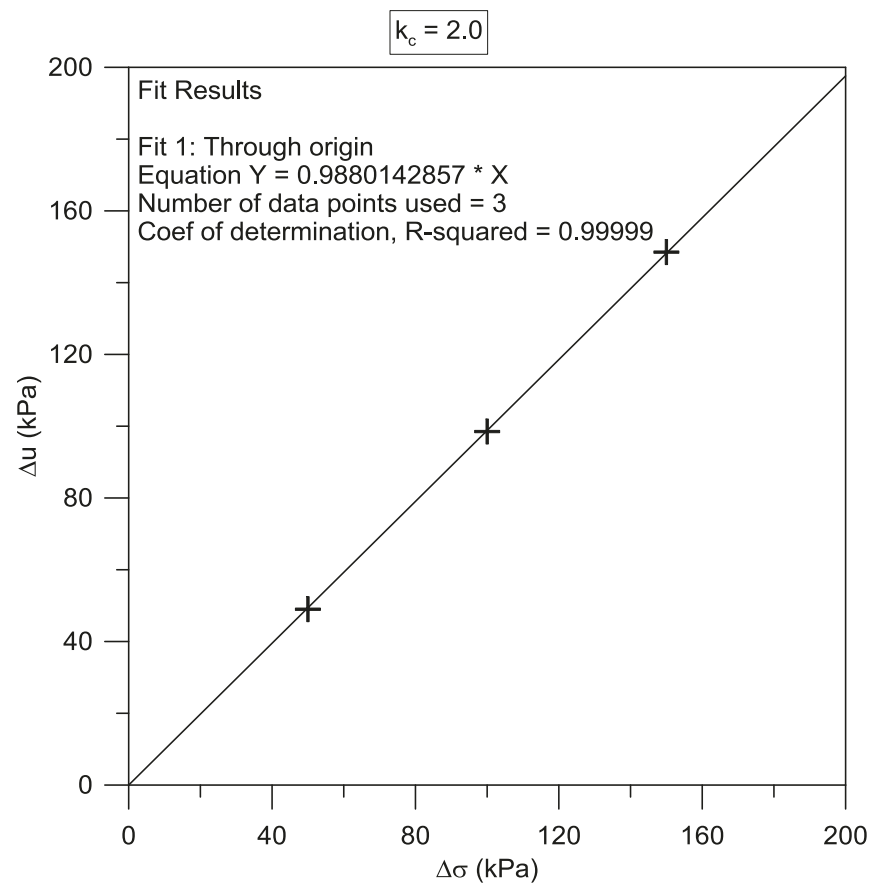

Fig. 6.2 Excess pore pressure induced during isotropic increment of principal stresses for $k_{c}=2.0$

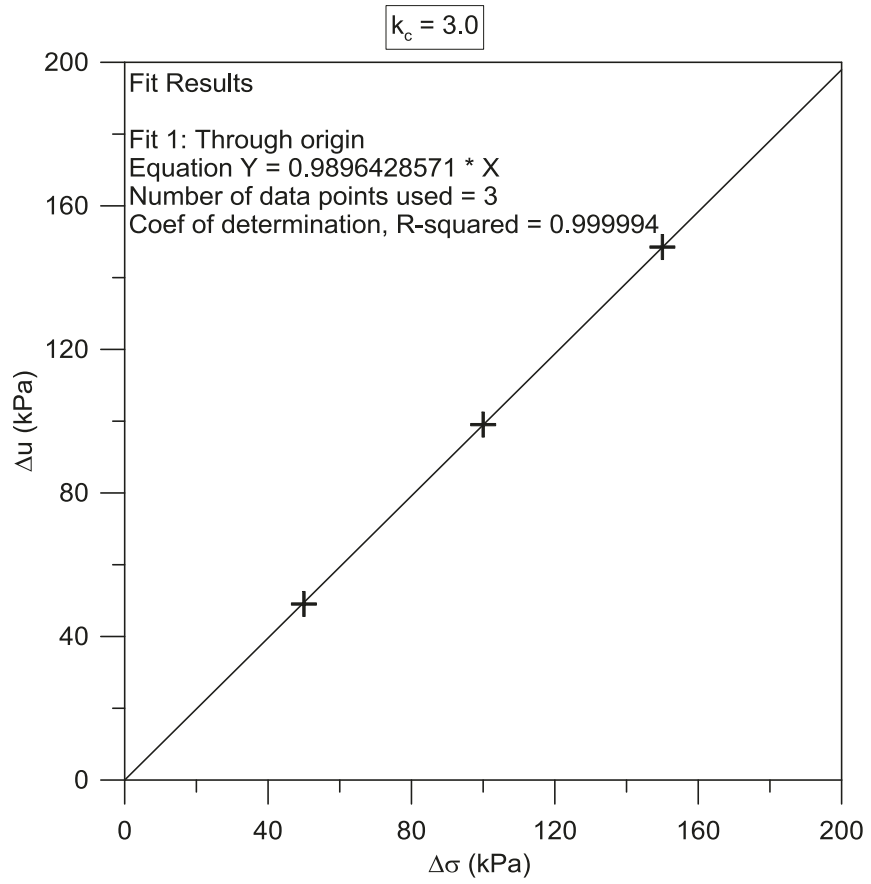

Fig. 6.3 Excess pore pressure induced during isotropic increment of principal stresses for $k_{c}=3.0$ 
Table 6.3 shows a summary of the $\Delta u / \Delta \sigma$ ratio measured in these tests and compares it to the Skempton's $B$ value under hydrostatic initial conditions. A Skempton's $B$ value of 0.994 (and 0.997) under an initial hydrostatic load increment is a clear indication that the specimens were well-saturated initially. As noted by Black and Lee (1973) Skempton's $B$ value in fully saturated "medium-soft" soils is 0.9988 and "stiff" soils is 0.9877 . Loosest deposited Fraser river sand can be considered to fall in between these two groups, and the measured $B$ value indicates that the specimens are probably fully saturated. Even if the specimens are fully saturated, the measuring system (tubing, transducers etc.) introduce some compliance and thus the measured value will be somewhat lower than that calculated using the theory, if the compressibility of water and the soil skeleton are known. In addition, measurement errors due to the electrical noise inherently present in any $D A Q$ system are responsible for some of the variation. The reported $B_{3 D} / B_{I S O}$ values are essentially 1 in all cases and clearly indicate that the initial three dimensional stress state does not influence pore pressure generation.

It should be noted that the stress increment did not cause any change to the deviatoric (or octahedral shear) stress and excess pore pressure generation was solely due to all around pressure increase. In other words, since the deviatoric stress increment was zero during all of these tests, and thus there was no shear induced excess pore pressure generation (i.e. pore pressure parameters " $A$ " and " $a$ " need not to be considered).

These results demonstrate that the Skempton's proposal to determine the pore pressure generated due to a hydrostatic stress increment is valid under three-dimensional initial loading conditions regardless of the value of the intermediate principal stress and the inclination of major principal stress, even in cross-anisotropic materials. 
Table 6.3 Comparison of pore pressure generation under hydrostatic and 3D initial stress conditions

\begin{tabular}{|c|c|c|c|c|}
\hline \multicolumn{3}{|c|}{ Initial stress state } & \multirow[b]{2}{*}{$\begin{array}{c}B_{I S O} \\
(\text { Skempton's } B)\end{array}$} & \multirow[b]{2}{*}{$\frac{B_{3 D}}{B_{I S O}}$} \\
\hline$\alpha_{c}$ & $b_{c}=\frac{\left(\sigma_{2 c}-\sigma_{3 c}\right)}{\left(\sigma_{1 c}-\sigma_{3 c}\right)}$ & $k_{c}=\frac{\sigma_{1 c}^{\prime}}{\sigma_{3 c}^{\prime}}$ & & \\
\hline $0^{\circ}$ & 0.4 & 1.5 & \multirow{3}{*}{0.994} & 0.998 \\
\hline $0^{\circ}$ & 0.4 & 2.0 & & 0.994 \\
\hline $0^{\circ}$ & 0.4 & 3.0 & & 0.994 \\
\hline $45^{\circ}$ & 0.4 & 1.3 & \multirow{4}{*}{0.997} & 1.000 \\
\hline $45^{\circ}$ & 0.4 & 1.5 & & 0.987 \\
\hline $45^{\circ}$ & 0.4 & 1.8 & & 0.990 \\
\hline $45^{\circ}$ & 0.4 & 2.0 & & 0.989 \\
\hline
\end{tabular}




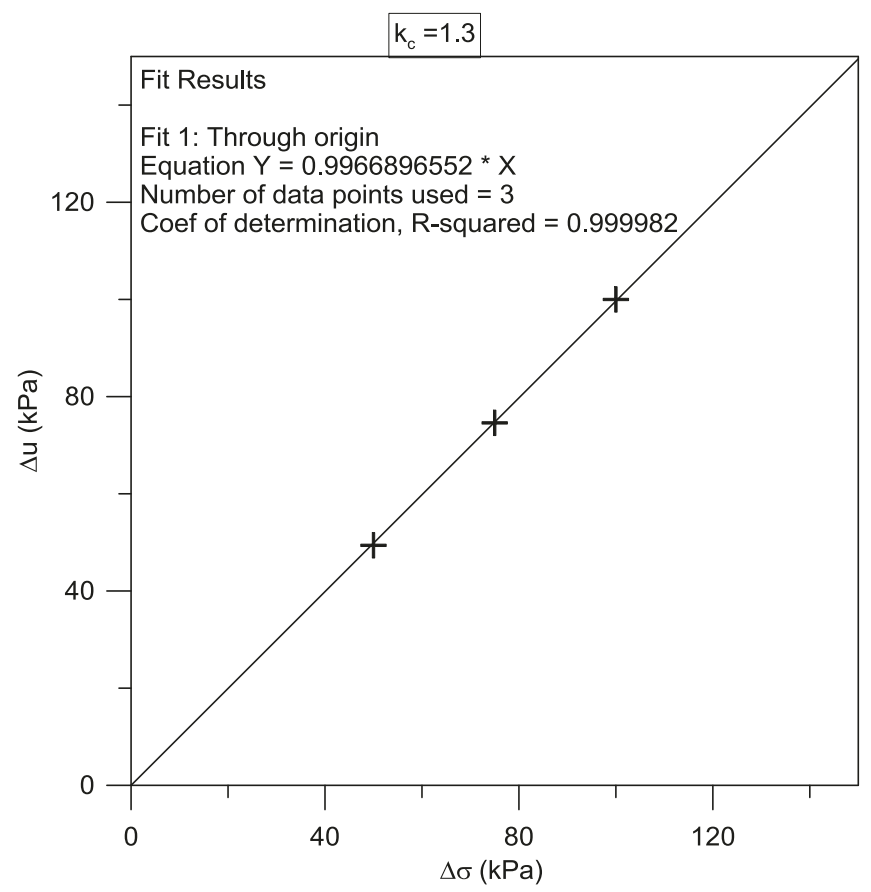

Fig. 6.4 Excess pore pressure induced during isotropic increment of principal stresses for $k_{c}=1.3$

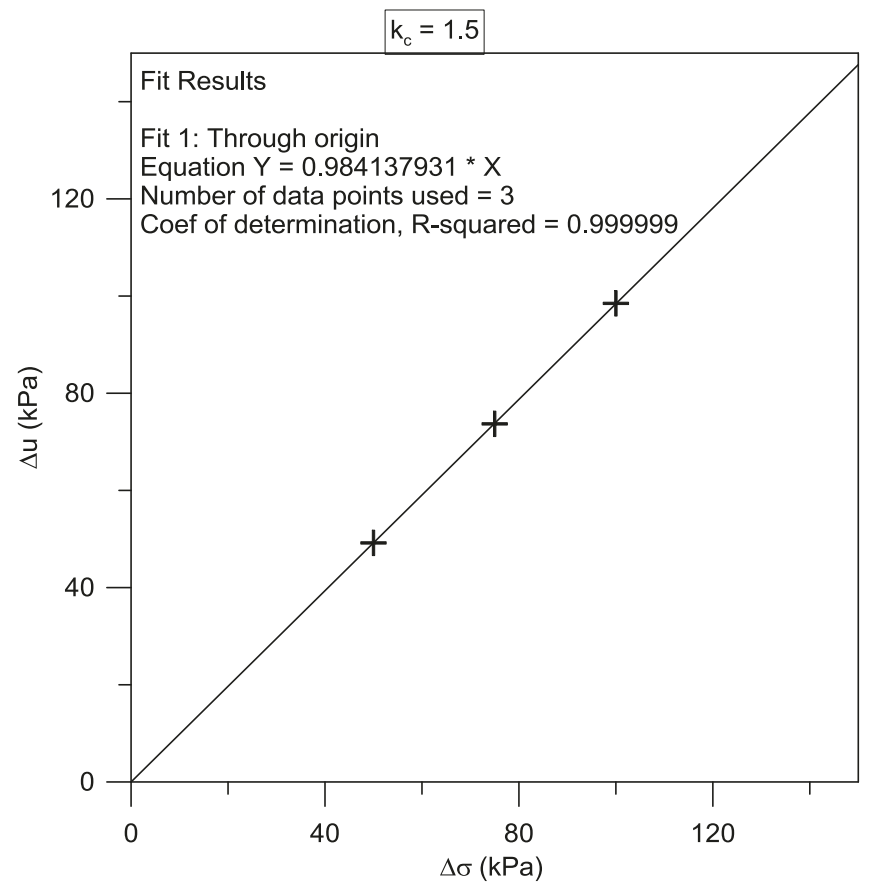

Fig. 6.5 Excess pore pressure induced during isotropic increment of principal stresses for $k_{c}=1.5$ 


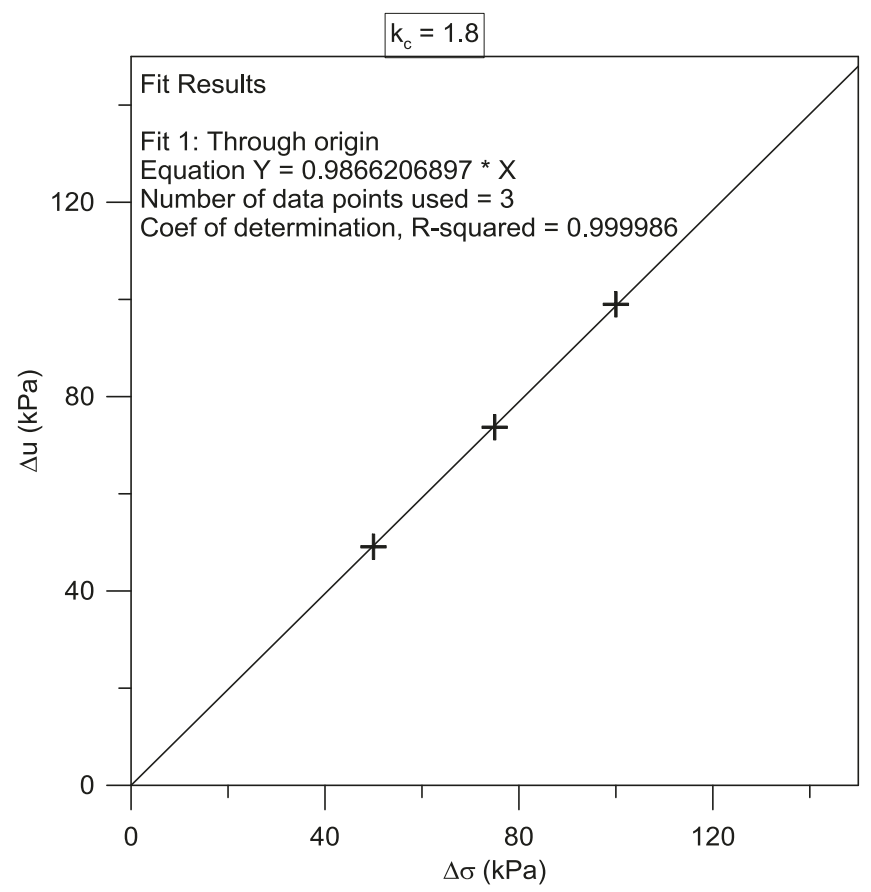

Fig. 6.6 Excess pore pressure induced during isotropic increment of principal stresses for $k_{c}=1.8$

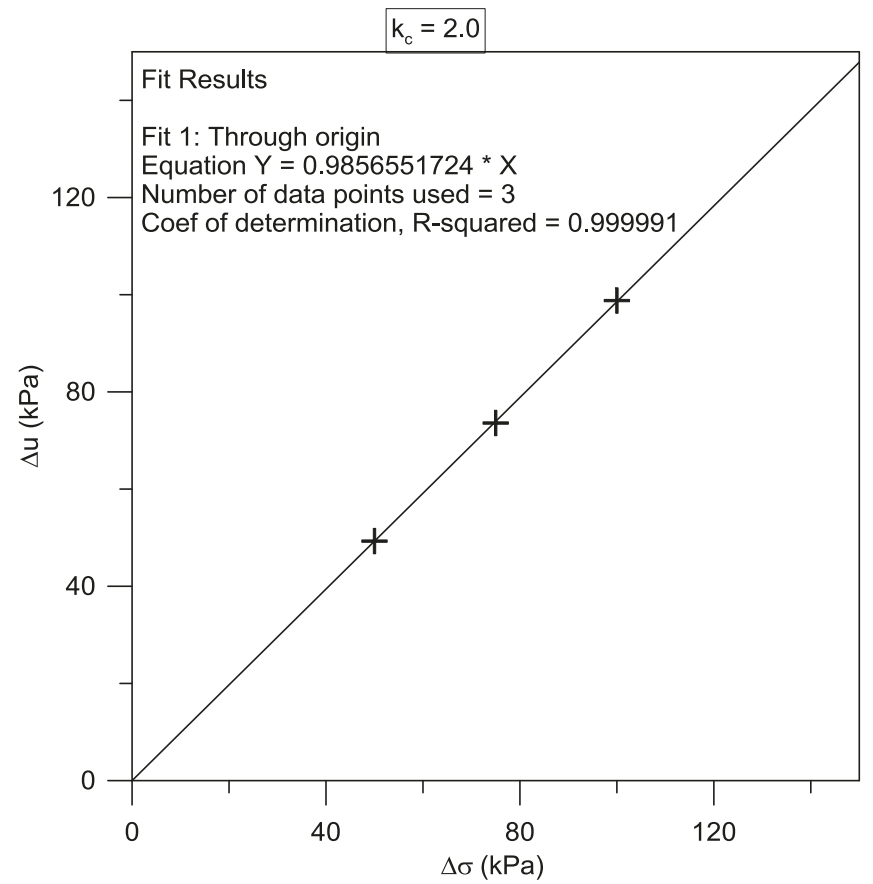

Fig. 6.7 Excess pore pressure induced during isotropic increment of principal stresses for $k_{c}=2.0$ 


\subsection{SHEAR INDUCED PORE PRESSURE GENERATION:}

\section{Skempton's A \& Henkel's a}

Fig. 6.8 shows the variation of excess pore water pressure induced by the two sets of total stress increments that introduce additional shear stresses $\left(\Delta \sigma_{1}=25 \mathrm{kPa}, \Delta \sigma_{2}=17.5 \mathrm{kPa}\right.$, $\Delta \sigma_{3}=12.5 \mathrm{kPa}$ and $\left.\Delta \sigma_{1}=100 \mathrm{kPa}, \Delta \sigma_{2}=70 \mathrm{kPa}, \Delta \sigma_{3}=50 \mathrm{kPa}\right)$ for three different direction of major principal stresses at the end of consolidation. In all three cases, shear induced pore water pressure decreased with an increase in initial shear stress. Further pore water pressure was increased by almost four times when the deviatoric stress was increased by for times. Since all the stress increments were applied at a specific $k_{c}$ in order to get a single representative value, the average pore water pressure induced by these two set of stress increments are considered in the following discussions.

Fig. 6.9 shows the variation of excess pore pressure induced by a non-hydrostatic stress increment of $\Delta \sigma_{1}=25 \mathrm{kPa}, \Delta \sigma_{2}=17.5 \mathrm{kPa}$ and $\Delta \sigma_{3}=12.5 \mathrm{kPa}$ at different initial $k_{c}$ values. The generated excess pore water pressure varied between about $16.1 \mathrm{kPa}$ and $18.6 \mathrm{kPa}$. For the test where the direction of the major principal stress was along the vertical axis $\left(\alpha_{\sigma}=0^{\circ}\right)$ the excess pore water pressure generated was $17.8 \mathrm{kPa}, 16.8 \mathrm{kPa}$ and $16.1 \mathrm{kPa}$ for the cases of $k_{c}=1.0,1.5$ and 2.0 respectively. On the other hand when the direction of the major principal stress was along the horizontal axis $\left(\alpha_{\sigma}=90^{\circ}\right)$ the excess pore water pressure generated for $k_{c}=1.0,1.5$ and 2.0 was $18.6 \mathrm{kPa}, 17.8 \mathrm{kPa}$ and $17.1 \mathrm{kPa}$ respectively. The third series of tests conducted at a principal stress inclination of $\alpha_{\sigma}=45^{\circ}$ induced $18 \mathrm{kPa}, 17 \mathrm{kPa}$ and $16.3 \mathrm{kPa}$ excess pore water pressure at $k_{c}=1.0,1.5$ and 2.0 respectively. The least amount of excess pore water pressure was developed in cases when the principal stress was aligned with the deposition direction and the most when it aligned with the bedding planes. These measurements suggest a 
systematic increase in pore water pressure generation with the increase in inclination of major principal stress to deposition direction, and a decrease in pore water pressure with the increase in initial static shear or effective stress ratio.
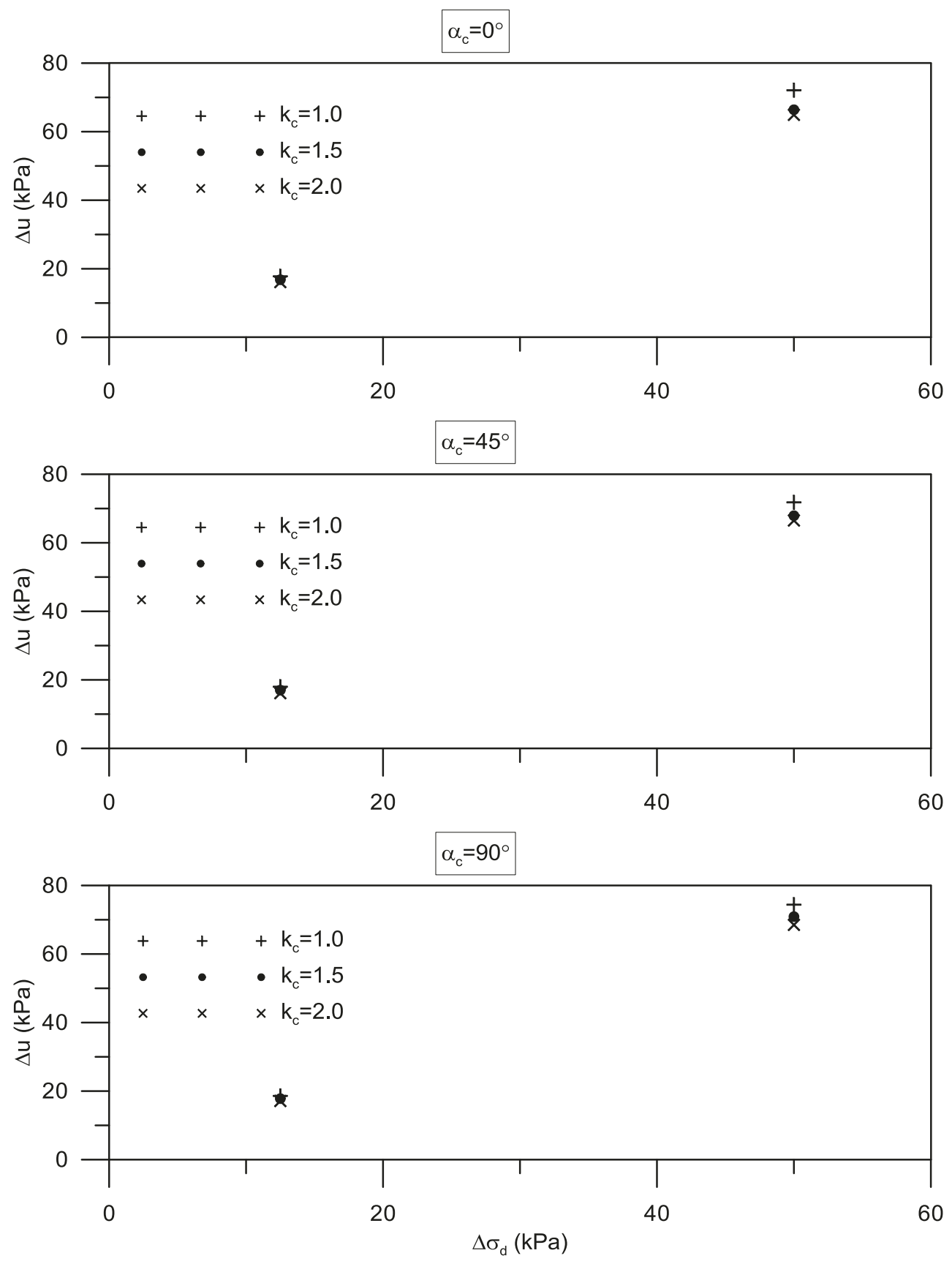

Fig. 6.8 Variation of pore water pressure with magnitude of deviatoric stress 


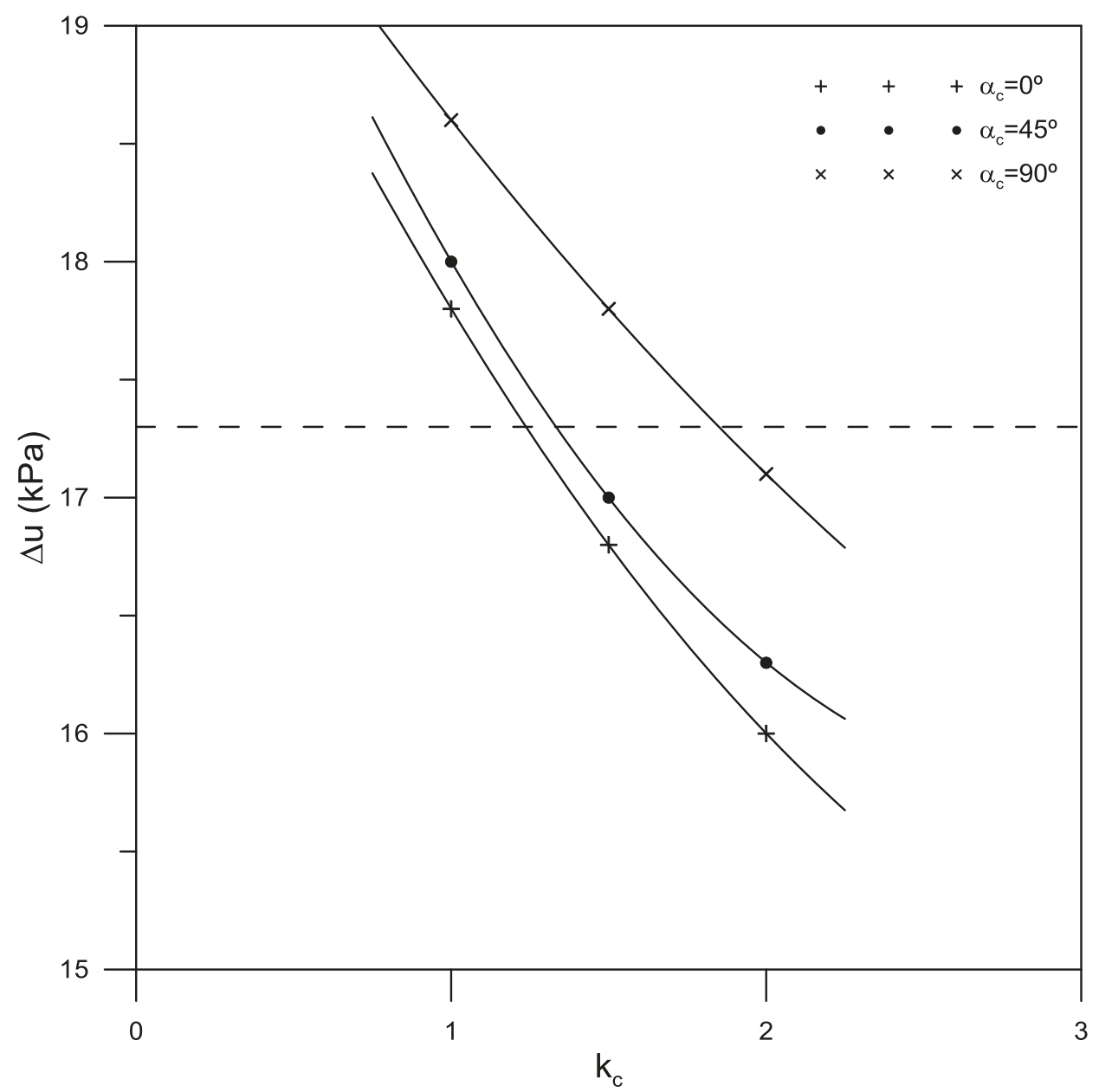

Fig. 6.9 Induced excess pore water pressure during anisotropic principal stress increment

The applied three dimensional stress increment can be considered as the sum of spherical (all around) and deviatoric stress components

$$
\begin{gathered}
{[\overline{\Delta \sigma}]=\left[\overline{\Delta \sigma}_{\text {SPHERICAL }}\right]+\left[\overline{\Delta \sigma}_{\text {DEVIATORIC }}\right]} \\
{\left[\begin{array}{ccc}
\Delta \sigma_{1}=25.0 & 0 & 0 \\
0 & \Delta \sigma_{2}=17.5 & 0 \\
0 & 0 & \Delta \sigma_{3}=12.5
\end{array}\right]}
\end{gathered}
$$

Based on the findings reported in the previous section, since the soil was fully saturated the hydrostatic stress increment of $\Delta \sigma=12.5 \mathrm{kPa}$ would have produced an essentially equivalent 
amount of excess pore water pressure, and thus the component of the excess pore pressure induced by the deviatoric stress increment, $\Delta u_{\text {shear induced }}=(\Delta u-12.5) \mathrm{kPa}$. The data presented above thus indicates $\Delta u_{\text {shear induced }}$ varied from about 3.6 to $6.1 \mathrm{kPa}$ depending on the value of $k_{c}$ and the direction of the major principal stress. Using Skempton's formulation, this would yield values of $A$ in the range 0.29 to 0.49 . These $A$ values are always positive and thus consistent with the expected contractive tendency of the material at this stage of loading.

On the other hand, the non-hydrostatic stress increment can also be considered to be the sum of all the around mean normal stress vector plus the stress difference vector as shown below

$$
\begin{aligned}
& {[\overline{\Delta \sigma}]=\left[\overline{\Delta \sigma}_{\text {MEAN NORMAL }}\right]+\left[{\overline{\Delta \sigma_{\text {DIFFERENCE }}}}\right]} \\
& {\left[\begin{array}{ccc}
\Delta \sigma_{1}=25.0 & 0 & 0 \\
0 & \Delta \sigma_{2}=17.5 & 0 \\
0 & 0 & \Delta \sigma_{3}=12.5
\end{array}\right]=\left[\begin{array}{ccc}
\Delta \sigma_{m}=18.3 & 0 & 0 \\
0 & \Delta \sigma_{m}=18.3 & 0 \\
0 & 0 & \Delta \sigma_{m}=18.3
\end{array}\right]} \\
& +\left[\begin{array}{ccc}
\Delta \sigma_{1}-\Delta \sigma_{m}=6.7 & 0 & 0 \\
0 & \Delta \sigma_{2}-\Delta \sigma_{m}=-0.8 & 0 \\
0 & 0 & \Delta \sigma_{3}-\Delta \sigma_{m}=-5.8
\end{array}\right]
\end{aligned}
$$

Considering Henkel's formulation, the excess pore water pressure generated due to a mean normal stress increment of $18.3 \mathrm{kPa}$ in a saturated soil would be $18.3 \mathrm{kPa}$. Thus the magnitude of the shear induced excess pore pressure $\Delta u_{\text {shear induced }}$ would vary from about $-2.2 \mathrm{kPa}$ to $+0.3 \mathrm{kPa}$ in the tests. Clearly, the excess pore pressure induced by the deviatoric stress is positive in some cases and negative in some cases depending on the value of $k_{c}$ and the direction of the major principal stress. It must, however, be noted that at this stage of the loading the soil has a contractive tendency, and a negative shear-induced pore pressure parameter is counter-intuitive since the contractive tendency would be expected to produce a positive increase in pore water pressure. 
Fig. 6.10(a) shows the variation of Skempton's ' $A$ ', and Henkel's ' $a$ ' parameters (that are normally used to quantify shear induced pore pressure) with effective principal stress ratio for the three different major principal stress directions. The shear induced excess pore water pressure in this material under these conditions is always positive in Skempton's formulation, and thus Skempton's pore pressure parameter " $A$ " is positive, and it decreases with the increasing amount of initial shear stress (or $k_{c}$ ) and increases with inclination of major principal stress with vertical. But the excess pore pressure induced by the deviatoric stress in Henkel's formulation is positive in some cases, and negative in others and thus the pore pressure parameter " $a$ " defined by Henkel (1960) and Henkel and Wade (1966) may be positive or negative, even though the normally consolidated material was tested at the loosest deposited state. The gradual reduction in Skempton's ' $A$ ', and Henkel's ' $a$ ' with increasing initial static shear stress (or $k_{c}$ ) is logical since the soils with higher initial $k_{c}$ require smaller increase in excess pore pressure to reach the failure line. Data reported by Tripathi (2005) from triaxial compression tests show similar reduction in Skempton's $A$ value as initial shear stresses increase. Even though the actual $A$ values are different due to different specimen and stress states, tests reported herein show a $28 \%$ reduction in $A$ value as $\mathrm{k}_{\mathrm{c}}$ increases from 1 to 2 , and Tripathi reports $37 \%$ reduction. Fig. 6.10(b) shows the variation of Skempton's ' $A$ ', and Henkel's ' $a$ ' parameters with major principal stress directions for the three different effective principal stress ratio. The systematic increase in pore pressure parameters (Skempton's ' $A$ ', and Henkel's ' $a$ ') with an increase in the inclination of the major principal stress to the deposition direction $\left(\alpha_{\sigma}\right)$ can be explained by the inherent anisotropy found in water deposited sands (Oda 1972). The sand exhibits highest strength and lowest deformability when major principal stress is aligned along the deposition direction $\left(\alpha_{\sigma}=0^{\circ}\right)$ and lowest strength and highest deformability when major principal stress is aligned 
along the bedding plane $\left(\alpha_{\sigma}=90^{\circ}\right)$. This behaviour is caused by higher pore pressure when the major principal stress is oriented along the bedding plane and lower pore pressure when the major principal stress is oriented along the deposition direction, thus higher pore pressure parameter for $\alpha_{\sigma}=90^{\circ}$ than $\alpha_{\sigma}=0^{\circ}$.
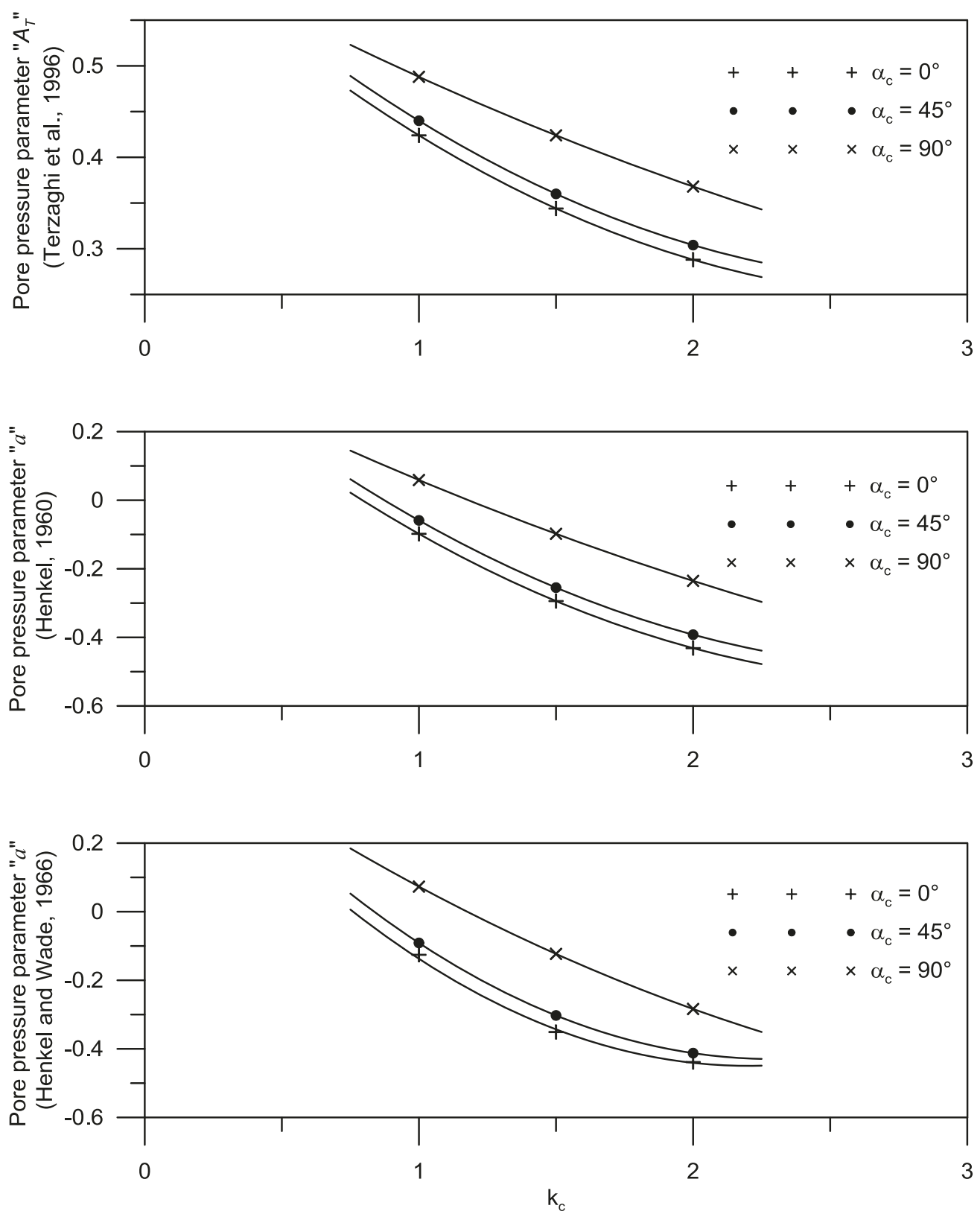

Fig. 6.10(a) Variation of pore water pressure parameters during anisotropic principal stress increment 

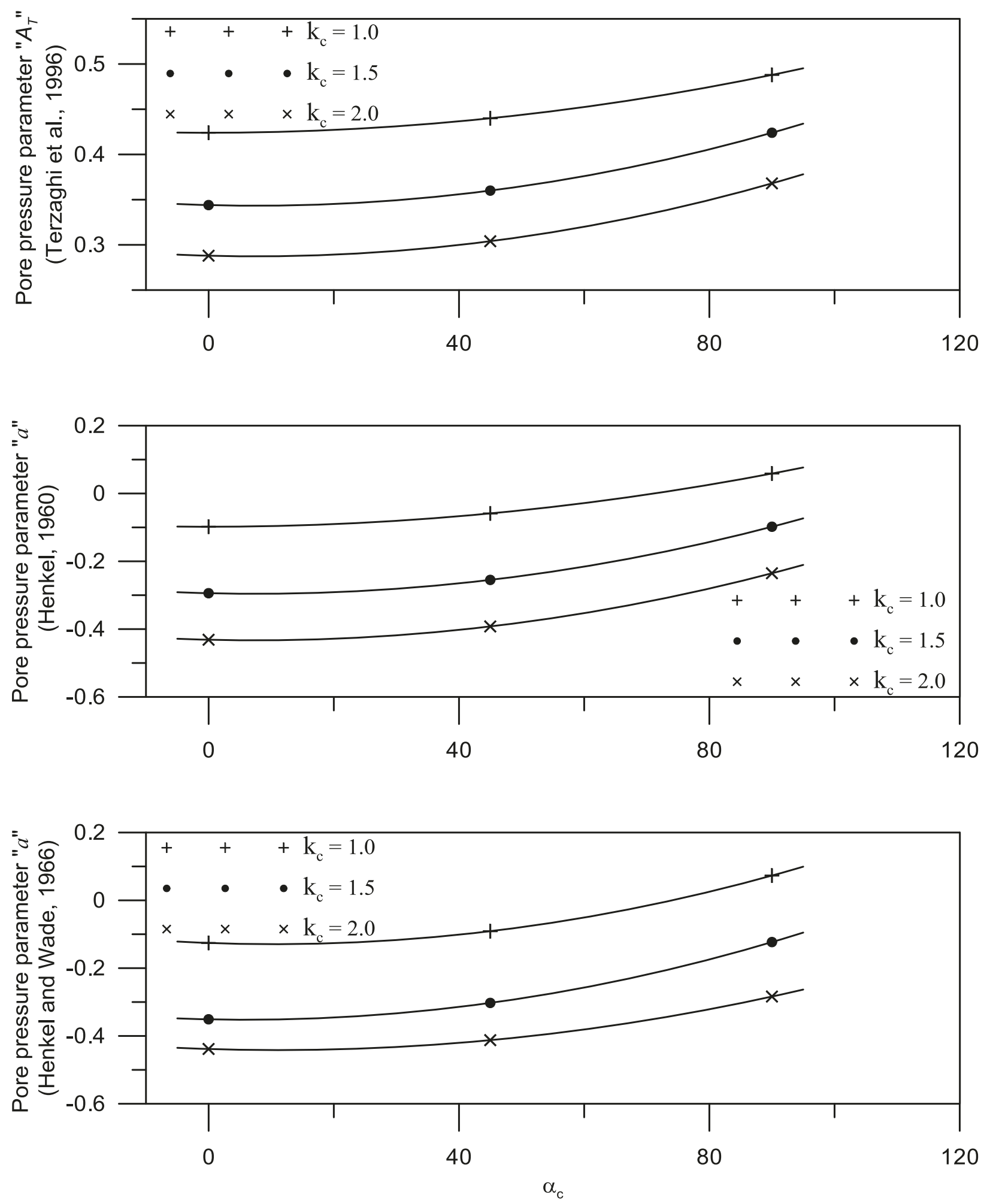

Fig. 6.10(b) Variation of pore water pressure parameters during anisotropic principal stress increment 
Test data published in the literature (Uthayakumar, 1996, Logeswaran, 2010) can also be used to validate this hypothesis. Fig. 6.11 is derived from the test data reported by Logeswaran (2010) and shows the influence of principal stress direction $\left(\alpha_{\sigma c}\right)$ on pore water pressure generation at a constant $\sigma_{m c}^{\prime}, b_{c}$ and $k_{c}$ in an HCT test. The specimens were consolidated to three different initial static shear stress states $\left(k_{c}=1.0,1.5\right.$ and 2.0 $)$ and $\sigma_{m c}^{\prime}=200 \mathrm{kPa}$. The specimens were sheared at a constant $b_{c}=0.4$, but in one case along the deposition direction and at $\alpha_{\sigma c}=0^{\circ}$ and $90^{\circ}$. The excess pore pressure response for $\alpha_{\sigma c}=0^{\circ}$ demonstrates that the sand initially deforms contractively up to about $3 \%$ shear strain, and subsequently dilates after reaching peak pore pressure whereas for $\alpha_{\sigma c}=90^{\circ}$ the sand generates relatively higher excess pore pressure, which was positive throughout. The increased tendency for more contrastive response at $\alpha_{\sigma c}=90^{\circ}$ than at $\alpha_{\sigma c}=0^{\circ}$ is well known. These results also show that the pore water pressure increases with the increasing direction of major principal stress. This is consistent with the test results achieved for this research program.

Fig. 6.12 illustrates the influence of principal stress direction $\left(\alpha_{\sigma c}\right)$ on Skempton's " $A$ " value obtained from the HCT test data shown in the previous figure. The result shows that the pore water pressure parameter increases with the increase in $\alpha_{\sigma c}$ and the trend of the variation is similar to that derived from the experiments carried out in this research program. 

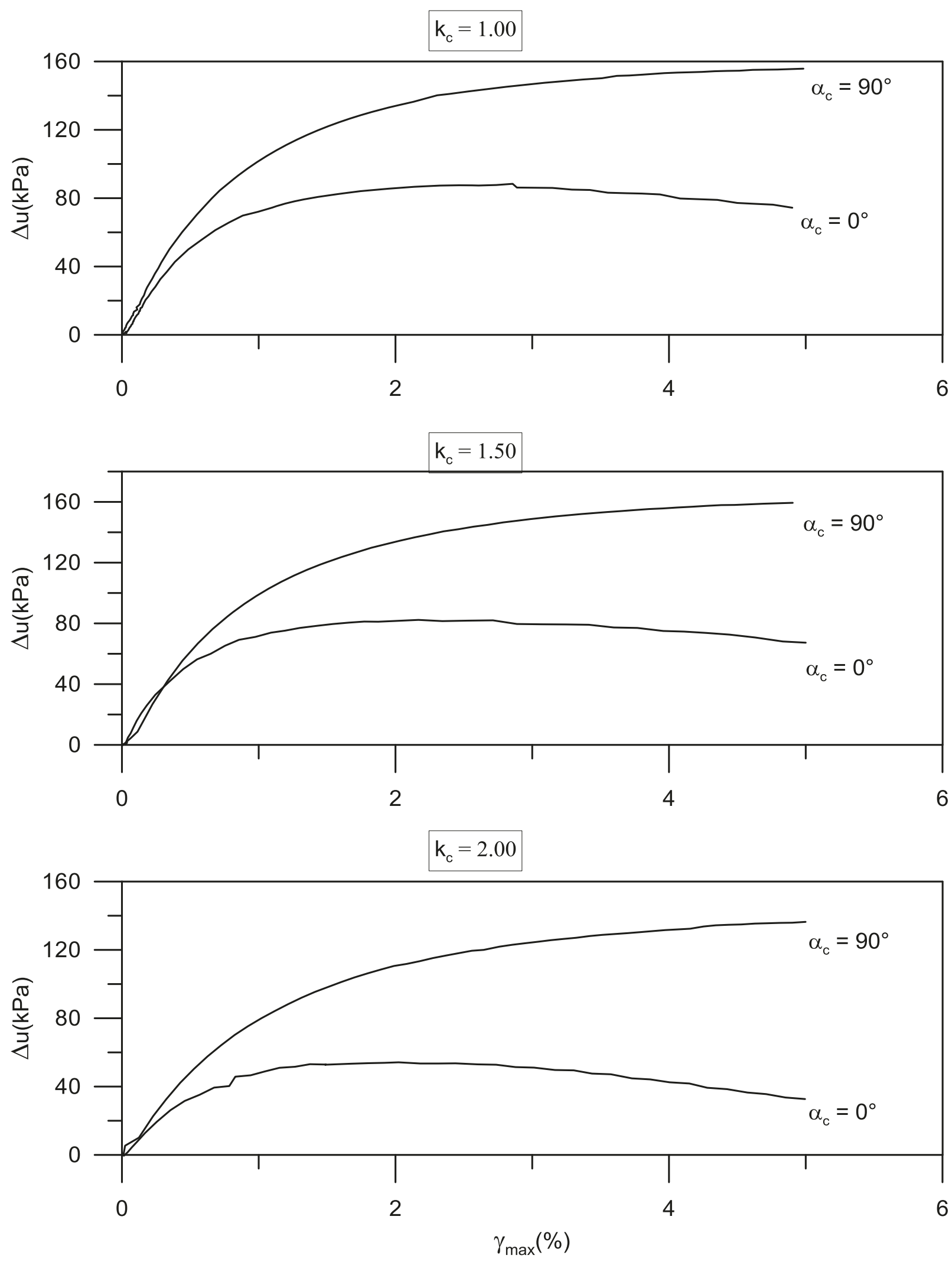

Fig. 6.12 Influence of principal stress direction on pore pressure generation for $k_{c}=1.0,1.5,2.0$ 

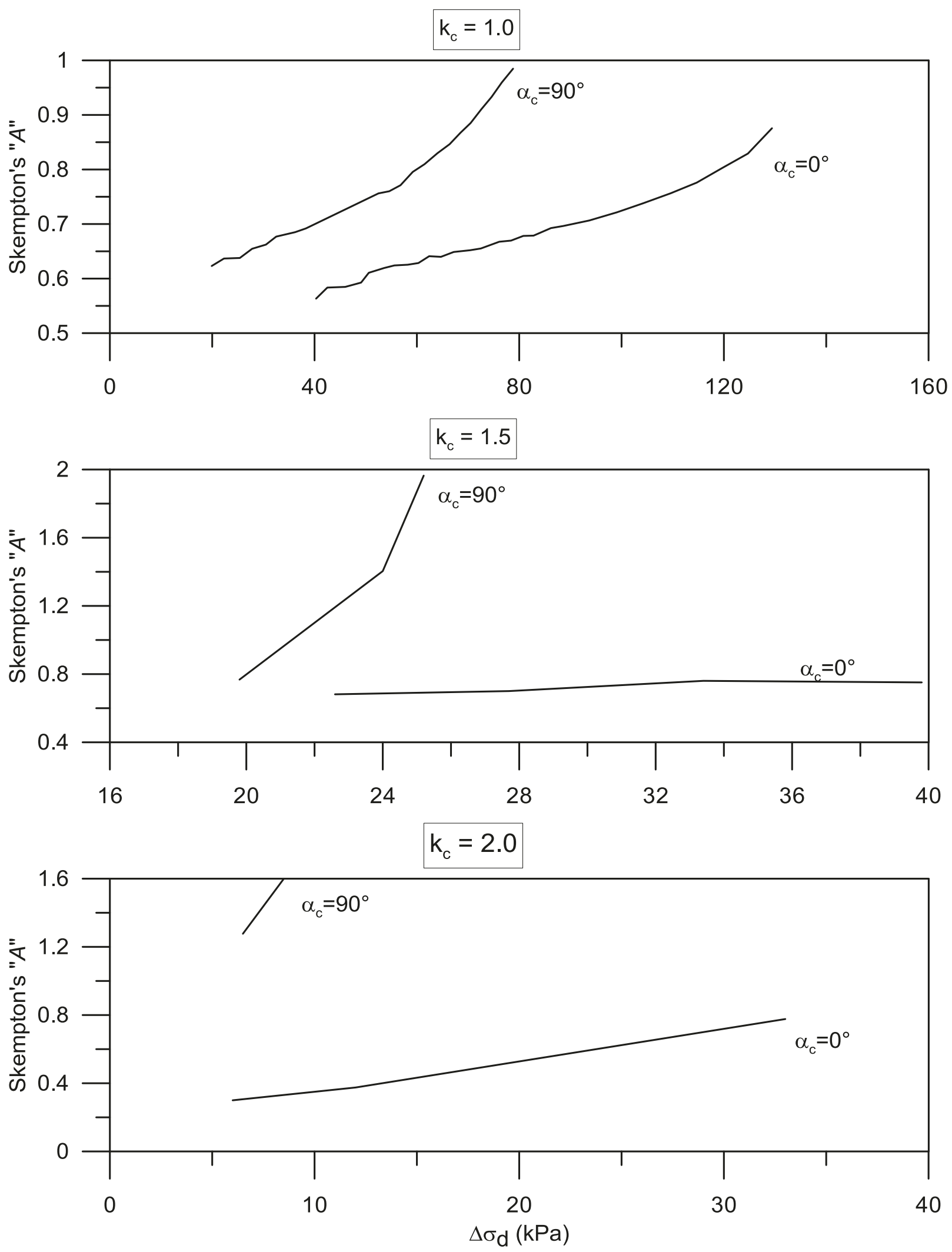

Fig. 6.13 Influence of principal stress direction on pore pressure parameter for $k_{c}=1.0,1.5,2.0$ 


\subsection{DISCUSSION}

A study aimed to better understanding the mechanics of the generation of excess pore water pressure under generalized three dimensional loading conditions is presented in this chapter. The main objective was to evaluate the applicability of the Skempton (1954) and Henkel (1960) formulations during generalized undrained loading. Prior experimental attempts to validate those formulations were limited to simpler loading modes only (Law and Holtz, 1978). Experiments were carried out on the loosest deposited Fraser River sand in the present study using the automated HCT device at different initial states.

An assessment of the Skempton's formulation for shear induced pore pressure generation indicates that the $A$ value is a function of the initial stress state (both the initial effective stress ratio, and the direction of the principal stresses). The computed $A$ value varied between about 0.3 to 0.5 for the given load increment, and its sign was consistent with the expected contractive response of the material. Given these findings, it can be concluded that the experimental evidence shows that Skempton's approach can be applied for generalized three dimensional loading conditions where the specimen was consolidated to a generalized stress state with different major, intermediate and minor principal stress and an inclination of major principal stress with vertical.

An evaluation of the Henkel's formulation, on the other hand, shows that Henkel's ' $a$ ' parameter that accounts for shear induced pore pressure generation may either be positive or negative depending on the value of $k_{c}$ and the direction of the major principal stress even when the sand was deposited at the loosest state and has contractive tendencies at the given stressdensity state. This is not a desired outcome, since the shear induced pore water pressure would be positive in such contractive material. More comprehensive studies may be needed to make a 
firm recommendation, but the initial results suggest that Henkel's formulation is not appropriate under generalized loading, even though it was intended for such loading (but validated only under simpler triaxial loading). 


\section{SUMMARY AND CONCLUSIONS}

Potential risk of soil liquefaction is a major concern in geotechnical design in regions that are susceptible to earthquake loading. Even though static liquefaction itself is a concern in loose sands, earthquake loading can trigger liquefaction in both loose and dense sands. Liquefaction potential of sand depends on various state parameters including relative density, soil fabric, stress/strain history, initial stress state and loading path. The basic understanding of this phenomenon and the effects of various factors controlling it have been derived from controlled laboratory experiments. But, the effects of principal stress rotation, and three dimensional stress states on pore pressure generation and liquefaction potential have not been fully understood to date.

\subsection{PRINCIPAL STRESS ROTATION \& LIQUEFACTION}

A systematic study was undertaken to assess the effects of principal stress rotation during cyclic and monotonic loading on liquefaction susceptibility. Isotropically consolidated soil specimens at initial principal stress direction of $\alpha_{\sigma c}=0^{\circ}, 45^{\circ}$ and $90^{\circ}$ were sheared monotonically under plane strain condition with a fixed rate of stress rotation given by $\Delta \sigma_{d} / \Delta \alpha_{\sigma}=8.89 \mathrm{kPa} /$ degree. In addition, monotonic tests were also conducted on isotropically consolidated soil specimens at a fixed initial principal stress direction of $\alpha_{\sigma c}=45^{\circ}$ but with different levels of stress rotation.

The soil specimens consolidated to an effective mean normal stress $\left(\sigma_{m c}^{\prime}\right)$ of $200 \mathrm{kPa}$ and initial effective stress ratio $\left(k_{c}\right)$ of 1.0 were subjected to cyclic shear with principal stress rotation about different initial principal stress directions given by $\alpha_{\sigma c}=0^{\circ}, 30^{\circ}, 45^{\circ}, 60^{\circ}$, and $90^{\circ}$. Most of the tests were conducted under plain strain conditions (intermediate stress 
parameter $b_{c}=0.4$ ), and a limited number of tests were conducted under axisymmetric conditions. All cyclic tests were conducted with a cyclic stress ratio (CSR) of 0.2 but with different level of principal stress rotations $\left(\Delta \alpha_{\sigma}=0^{\circ}, 30^{\circ}, 45^{\circ}, 60^{\circ}\right.$, and $\left.90^{\circ}\right)$. Principal stresses in cyclic tests were rotated in a smooth manner to reflect the true field conditions. The largest inclination of the direction of principal stresses with respect to the initial state coincided with the peak cyclic shear stress during loading.

The initial direction and degree of stress rotation during shear plays an important role on the undrained behaviour of sands. The results of the monotonic tests supplement the findings reported in the literature, and indicate that for a given initial direction, higher degree of stress rotation (that further aligns the major principal stress toward the bedding plane) will lead to weaker response. Similarly, when the rate of stress rotation was fixed, specimens for which the major principal stress was oriented more closely to the horizontal bedding plane yielded relatively weaker response compared to the soil specimen for which the major principal stress was oriented more closely to the vertical deposition direction.

The tests carried out to study the influence of level of rotation on cyclic liquefaction resistance of Fraser River sand specimen clearly indicate that principal stress rotation strongly influences the cyclic strength of the sand.

- Strongest response was obtained when there was no principal stress rotation nor stress reversal $\left(\Delta \alpha_{\sigma}=0^{\circ}\right)$. In fact, none of the specimens under this condition liquefied even after 60 cycles of loading.

- Irrespective of the initial principal stress direction, the lowest cyclic resistance was obtained in tests in which the principal stresses rotated by $\pm 45^{\circ}$ about its initial position. This finding is consistent with the finding reported in the literature based on a limited 
number of tests for initial principal stress direction oriented along the vertical deposition direction.

- As indicated in the literature, the direction of major principal stress with respect to the bedding plane also plays an important role in determining the cyclic liquefaction resistance of the sand specimen. The sand exhibits higher cyclic resistance when the rotation of principal stress is closer to the deposition direction $\left(\alpha_{\sigma c}=30^{\circ}\right.$ and $\Delta \alpha_{\sigma}=$ $30^{\circ}$ ) whereas the cyclic resistance reduces drastically when the rotation of principal stress is further towards the bedding plane $\left(\alpha_{\sigma c}=30^{\circ}\right.$ and $\left.\Delta \alpha_{\sigma}=60^{\circ}\right)$.

- The magnitude of the maximum torsional shear applied on the horizontal plane and the time history of the torsional shear stress (especially the duration during which it remains close to the maximum value) also affects the cyclic resistance of the specimen.

The tests carried out to study the influence of initial direction of principal stress on cyclic liquefaction resistance of Fraser River sand specimen indicates that

- The cyclic resistance of sand depends on the magnitude of the maximum torsional shear stress applied on the horizontal plane $\left(\tau_{z \theta \max }\right)$ and the nature of the variation of torsional shear stress on the horizontal plane.

- For a given $\tau_{z \theta \max }$ weaker behaviour is observed when the direction of the major principal stress is aligned toward the weakest horizontal plane.

- In some specific scenarios, for example the behaviour of $\alpha_{\sigma c}=0^{\circ}$ and $90^{\circ}$ with rotation of $\Delta \alpha_{\sigma}=90^{\circ}$, the actual nature of principal stress rotation influences the behaviour of cyclic liquefaction resistance. 
In summary, the cyclic test results demonstrate that the lowest cyclic resistance always corresponds to $45^{\circ}$ stress-rotation (regardless of the initial principal stress direction). This suggests that the use of cyclic simple shear (SS) tests might be appropriate for design purposes. Even though the principal stress rotation in simple shear device is not controllable, a cyclic simple shear test subjects specimens to approximately $\pm 45^{\circ}$ stress rotation (but generally about the vertical direction, $\alpha_{\sigma c}=0^{\circ}$ ) and thus correspond to a conservative estimate. Even though it is not common practice, different initial $\alpha_{\sigma c}$ values can be simulated in SS tests by applying appropriate static shear stress on the horizontal plane. Further, SS also produces the plane strain condition which is representative of many geotechnical engineering problems.

\subsection{Pore Pressure Generation \& 3D stress state}

A series of experiments were carried out to investigate the applicability of the pore pressure formulations (Skempton, 1956, Henkel, 1960) for more generalized loading state using the automated HCT device. Soil specimens were anisotropically consolidated to a more generalized stress state compared to the typical triaxial stress conditions which have been used in the literature to validate the formulations. The initial stress states explored included initial nonhydrostatic stress states under plane strain loading, and even states with an inclination of major principal stress with vertical (that represents the stress conditions in slopes). Prescribed generalized total stress paths were applied to the specimens and the amount of pore pressure

generated was measured. The applicability of Skempton's $B$ value concept for the generalized loading and the effect of $k_{c}$ on the pore pressure parameters $A$ and $a$ were assessed. 
Skempton showed that an isotropic increment of principal stresses in a saturated soil generates pore pressure equal to the magnitude of the stress increments. The analytical formulation based on an isotropic media was validated experimentally based on triaxial test results. Most natural soils, especially those deposited in an alluvial environment are cross anisotropic in nature, and a generalized formulation relating principal stress increments and excess pore pressure generation in such cross anisotropic materials is provided in chapter 6 .

- The experimental evidence shows that Skempton's approach can be applied for generalized three dimensional loading conditions in cross anisotropic materials where the specimen was consolidated to a generalized stress state with different major, intermediate and minor principal stress and an inclination of major principal stress with vertical.

- The research reported suggests that the pore pressure parameters $A$ and $a$ decrease with an increase in the initial shear stress $\left(k_{c}\right)$. This can be explained by the fact that at a specific confining stress level, and for similar total stress increments, the amount of pore pressure required to reach the failure envelope decreases with an increase in the initial shear stress. This is the case in water deposited sands where the shear strength of the soil increases with the confining pressure.

- Both pore pressure parameters $A$ and $a$ increase with an increase in inclination of major principal stress with vertical. Thus, the effective stress at the critical/failure state would decrease with an increase in the inclination of major principal stress leading to weaker response as the principal stress aligns with the bedding plane. 


\subsection{RECOMMENDATIONS FOR FUTURE RESEARCH}

This thesis emphasizes the importance of generalized initials stress states, and stress rotation on the on the undrained behaviour of Fraser River sand. The research program investigated the behaviour of loosest deposited Fraser River sand at different initial principal stress inclinations $\left(\alpha_{\sigma c}\right)$ and different levels of stress rotation $\left(\Delta \alpha_{\sigma}\right)$. Further research in the following topics will enhance the findings reported in this thesis, and allow drawing generalized conclusions.

- Fraser River sand particles are predominantly has sub-angular. Extending this study to sands with different particle shapes would permit an assessment of the impact of grain shape on the undrained behaviour of sand while principal stress rotates. The widely used Ottawa sand, which is predominantly sub-rounded is an excellent candidate for further research.

- Most natural soils exist at non-hydrostatic initial states, and a study on the influence of initial non-hydrostatic stress state on the cyclic resistance of the sand under principal stress rotations will complement the current knowledge, and will have practical implications.

- Denser sands are generally less anisotropic than loose sands. Thus a detailed study on the effect of initial principal stress direction and level of principal stress rotation on liquefaction resistance of dense sands is also recommended. 


\section{REFERENCES}

Arthur, J.R.F., and Menzies, B.K., (1972), "Inherent Anisotropy in Sand”, Géotechnique, 22 (1): $115-128$.

Arthur, J.R.F., Chua, K.S., and Dunstan, T., (1977), "Induced Anisotropy in a Sand", Géotechnique, 27(1): 13-30.

Arthur, J.R.F., Chua, K.S., Dunstan, T., and del Camino, J.R., (1980), "Principal Stress Rotation: A Missing Parameter", Journal of the Geotechnical Engineering Division, ASCE, 106(4): 419-433.

ASTM. 2001a. Standard Test Methods for Maximum Index Density and Unit Weight of Soils Using a Vibratory Table (D-4253-00). Annual Book of ASTM Standards, American Society for Testing and Materials, Philadelphia, PA. Vol. 04.08.

ASTM. 2001b. Standard Test Methods for Minimum Index Density and Unit Weight of Soils and Calculation of Relative Density (D-4254-00). Annual Book of ASTM Standards, American Society for Testing and Materials, Philadelphia, PA. Vol. 04.08.

Braja M. Das (2008), “Advanced Soil Mechanics”, $3^{\text {rd }}$ Edition, New York, Taylor \& Francis Group, 594p.

Broms, B.B., and Casbarian, A.O., (1965), "Effects of Rotation of the Principal Stress Axes and of the Intermediate Principal Stress on the Shear Strength", Proceeding, $6^{\text {th }}$ International Conference on Soil Mechanics and Foundation Engineering, Vol. 1, pp. 179-183.

Casagrande, A., (1975), "Liquefaction and Cyclic Deformation of Sands: a Critical Review", Proceedings Fifth Pan-American Conference on Soil Mechanics and Foundation Engineering, pp. 79-133. 
Casagrande, A., and Carrillo, N., (1944), "Shear Failure of Anisotropic Materials", Journal of the Boston Society of Civil Engineers, 31(4) :122-135.

Castro, G., (1969), "Liquefaction of Sands", PhD Thesis, Harvard University, Cambridge, Massachusetts, 231p.

Castro, G., (1987), "On the Behaviour of Soils during the Earthquakes-Liquefaction", Proceedings of Soil Dynamics and Liquefaction, No. 42. Edited by A.S. Cakmak. Elsevier, pp. 169-204.

Castro, G., and Poulos, S.J., (1977), "Factors Affecting Liquefaction and Cyclic Mobility", Journal of Geotechnical Engineering Division, ASCE, 103(6) :501-516.

Castro, G., Poulos, S.J., France, J.W., and Enos, J.L., (1982), "Liquefaction Induced by cyclic Loading”, Report submitted to the National Science Foundation, March 1982.

Chern, J.C., (1985), “Undrained Response of Sands with Emphasis on Liquefaction and Cyclic Mobility", PhD Thesis, University of British Columbia, Vancouver, Canada, 213p.

Ha, D., (2003), "Effect of Initial Stress State on the Undrained Cyclic Behaviour of Sands", M.A.Sc. Thesis, Carleton University, Ottawa, Canada.

Haruyama, M., (1981), “Anisotropic Deformation-Strength Characteristics of an Assembly of Spherical Particles under 3-D Stresses", Soils and Foundations, 21(4) :41-55.

Henkel, D. J., (1960), "The Shear Strength of Saturated Remoulded Clays", Proceedings of the ASCE Research Conference on Shear Strength of Cohesive Soils, Boulder, Colo., New York, pp. 533-554.

Henkel, D.J., and Wade, N.H., (1966). "Plane Strain Tests on Remoulded Clay", Journal of the Soil Mechanics and Foundation Division, ASCE, Vo. 92(6):67-80. 
Hight, D.W., Gens, A., and Symes, M.J., (1983), “The Development of a New Hollow Cylinder Apparatus for Investigating the Effects of Principal Stress Rotation in Soils", Géotechnique, 33(4) :355-383.

Ishihara, K., (1993), "Liquefaction and Flow Failure during Earthquakes", Géotechnique, $43(3): 351-415$.

Ishihara, K., and Yamazaki, F., (1980), "Cyclic Simple Shear Tests on Saturated Sand in MultiDirectional Loading”, Soils and Foundations, 20(1):45-59.

Ishihara, K., Tatsuoka, F., and Yasuda, S., (1975), "Undrained Deformation and Liquefaction of Sands under Cyclic Stresses", Soils and Foundations, 15(1):29-44.

Kramer, S.L., and Seed, H.B., (1988), "Initiation of Soil Liquefaction under Static Loading Conditions", Journal of Geotechnical Engineering, ASCE, 114(4):412-430.

Kwan Lam, W., and Tatsuoka, F., (1988), "Effects of Initial Anisotropic Fabric and $\sigma_{2}$ on Strength and Deformation Characteristic of Sand", Soils and Foundations, 28(1), pp. 89106.

Lade, P.V., and Wasif, U., (1988), "Effect of Height- to- Diameter ratio in Triaxial Specimens on the Cross Anisotropic Sand", Advanced Triaxial Testing of Soil and Rock, ASTM STP 977, R.T. Donaghe, R.C. Chaney, and M.L. Silver, Eds., American Society for Testing and Materials, Philadelphia, ASCE, pp. 706-714.

Lambe, T. W., (1951), “Soil Testing for Engineers”, John Wiley \& Sons Inc., New York.

Law, K.T., and Holtz, R.D, (1978), “A Note on Skempton's A Parameter With Rotation of Principal Stresses", Géotechnique, 28(1):57-64.

Lee, K.L., and Seed, H.B., (1967), "Cyclic Stress Conditions Causing Liquefaction of Sand”, Journal of Geotechnical Engineering Division, ASCE, 93(5):47-70. 
Logeswaran, P., (2005), "Behaviour of Sands under Simultaneous Changes of Volume and Pore Pressure", MASc Thesis, Carleton University, Ottawa, Canada, 177p.

Logeswaran, P., (2010), "Behaviour of Sands under Generalized Loading and Drainage Conditions", PhD Thesis, Carleton University, Ottawa, Canada, 273p.

Logeswaran, P., and Sivathayalan, S., (2005), "Characterization of Response of Fraser River Sand under Simultaneous Change in Volume and Pore Pressure", $58^{\text {th }}$ Canadian Geotechnical Conference Saskatoon, Saskatchewan, Canada, Sept. 19-21, 2005.

Love, A. E. H., (1927), “The Mathematical Theory of Elasticity”, Cambridge University Press, Cambridge.

Manmatharajan, V., (2011), "Initial Stress State and Stress History Effects on Liquefaction Susceptibility of Sands", MASc Thesis, Carleton University, Ottawa, Canada, 182p.

Menzies, B.K., (1987), “A computer Controlled Hydraulic Testing System”, Proceedings, Symposium on Advanced Triaxial Testing, ASTM STP 977, Philadelphia, PA, Vol. 1, pp. 82-94.

Miura, K., Miura, S., and Toki, S., (1986), "Deformation Behaviour of Anisotropic Dense Sand under Principal Stress Axes Rotation", Soils and Foundations, 26(1):36-52.

Miura, K., Miura, S., and Toki, S., (1986), “Deformation Prediction for Anisotropic Sand during the Rotation of Principal Stress Axes", Soils and Foundations, 26(3):42-56.

Miura, S., and Toki, S., (1982), "A Sample Preparation Method and Its Effect on Static and Cyclic Deformation - Strength Properties of Sand", Soils and Foundations, 22(1):61-77.

Miura, S., and Toki, S., (1984), "Anisotropy in Mechanical Properties and Its Simulation of Sands from Natural Deposits", Soils and Foundations, 24(3):69-84. 
Mohamad, R., and Dobry, R., (1986), "Undrained Monotonic and Cyclic Triaxial Strength of Sand", Journal of Geotechnical Engineering, ASCE, 112(10):941-958.

Nakata, Y., Hyodo, M., Murata. H., and Yasufuku, N., (1998), "Flow Deformation of Sands Subjected to Principal Stress Rotation", Soils and Foundations, 38(2):115-128.

Negussey, D., Wijewickreme, W.K.D., and Vaid, Y.P., (1988), “Constant Volume Friction Angle of Granular Materials", Canadian Geotechnical Journal, 25, pp. 50-55.

Oda, M., (1972a), "Initial Fabrics and Their Relations to Mechanical Properties of Granular Material", Soils and Foundations, 12(1):17 -36.

Oda, M., (1972b), "The Mechanism Fabric Changes during Compressional Deformation of Sand", Soils and Foundations, 12(2):1-18.

Oda, M., (1981). “Anisotropic Strength of Cohesionless Sands”, Journal of the Geotechnical Engineering. Division., ASCE, 107(9):1219-1231.

Oda, M., Koishikawa, I., and Higuchi, T., (1978), "Experimental Study of Anisotropic Shear Strength of Sand by Plane Strain Test", Soils and Foundations, 18(1):25-38.

Oda, M., Nasser, S.N., and Konishi, J. (1985). "Stress-Induced Anisotropy in Granular Masses", Soils and Foundations, 25(3):85-97.

Saada, A.S., and Puccini, P., (1985), Discussion on "The Development of a New Hollow Cylinder Apparatus for Investigating the Effects of Principal Stress Rotation in Soils", Géotechnique, 35(1):78-85.

Sayao, A.S.F., (1989), "Behaviour of Sand under General Stress Paths in the Hollow Cylinder Torsional Device", PhD Thesis, University of British Columbia, Vancouver, Canada, 1996p. 
Sayao, A.S.F., and Vaid, Y.P., (1991), “A Critical Assessment of Stress Nonuniformities in Hollow Cylinder Test Specimens”, Soil and Foundations, 31(1):60-72.

Seed, H.B., (1979), "Soil Liquefaction and Cyclic Mobility Evaluation for Level Ground during Earthquakes”, Journal of Geotechnical Engineering Division, ASCE, 105(2):201-225.

Shahsavari Goughari, M., (2012), "Effect of Initial Shear Stress Direction and Stress History on the Undrained Behaviour of Sands under Triaxial Loading”, MASc Thesis, Carleton University, Ottawa, Canada, 173p.

Shibuya, S., (1988), "A Servo System for Hollow Cylinder Torsional Testing of Soils", Geotechnical Testing Journal, ASTM, 11(2):109-118.

Sivathayalan, S., (2000), "Fabric Initial State and Stress Path Effects on Liquefaction Susceptibility of Sands, PhD Thesis, University of British Columbia, Vancouver, Canada, $307 p$.

Sivathayalan, S., and Logeswaran, P., (2007), "Behaviour of Sands under Generalized Drainage Boundary Conditions", Canadian Geotechnical Journal, 44(2):138-150.

Sivathayalan, S., and Logeswaran, P., (2008), "Experimental Assessment of the Response of Sands under Shear-Volume Coupled Deformation", Canadian Geotechnical Journal, 45(9), pp.1310-1323.

Sivathayalan, S., and Vaid, Y.P., (2002), "Influence of Generalized Initial State and Principal Stress Rotation on the Undrained Response of Sands”, Canadian Geotechnical Journal, 39(1):63-76.

Sivathayalan, S., Manmatharajan, V., Logeswaran, P., (2011), "Hollow Cylinder Torsional Shear Tests to Evaluate the Role of Principal Stress Direction on Cyclic Resistance", $14^{\text {th }}$ PanAm Geotechnical Conference, Toronto, ON, Oct 1-6, 
Skempton, A.W., (1954), "The pore-pressure coefficients $A$ and B", Ge'otechnique, 4(4):143147.

Stedman, D., (1997), "Effects of Confining Pressure and Static Shear on Liquefaction Resistance of Fraser River Sand", MASc Thesis, University of British Columbia, Vancouver, Canada, 93p.

Symes, M.J., Gens, A. and Hight, D.W. (1984), "Undrained Anisotropy and Principal Stress Rotation in Saturated Sand", Géotechnique, 34(1):11-27.

Symes, M.J., Gens, A., and Hight, D.W., (1988), "Drained Principal Stress Rotation in Saturated Sand", Géotechnique, Vo. 38(1):59-81.

Symes, M.J., Hight, D.W., and Gens, A., (1982), "Investigating Anisotropy and the Effects of Principal Stress Rotation and of the Intermediate Principal Stress Using a Hollow Cylinder Apparatus", IUTAM Conference on Deformation and Failure of Granular Materials, Delft, pp. 441-449.

Symes, M.J., Shibuya, S., Hight, D.W. and Gens, A. (1985), "Liquefaction with Cyclic Principal Stress Rotation", Proceedings. $11^{\text {th }}$ International Conference on Soil Mechanics and Foundation Engineering, San Francisco, 4, pp. 1919-1922.

Taylor, D.W., (1948), "Fundamental of Soils mechanics” John Wiley \& Sons.

Terzaghi, K, Peck, R.B., and Mesri, G. (1996). "Soil Mechanics in Engineering Practice", $3^{\text {rd }}$ Edition, New York, John Wiley \& Sons Inc. p 504.

Terzaghi, K. and Peck, R.B., (1948), "Soil Mechanics in Engineering Practice", $2^{\text {nd }}$ edition, John Wiley and Sons, New York.

Thomas, J., (1992), "Static, Cyclic and Post Liquefaction Behaviour of Fraser River Sand", M.A.Sc. Thesis, University of British Columbia, Vancouver, Canada, 117p. 
Uthayakumar, M., (1995), "Liquefaction of Sands under Multi-axial Loading", PhD Thesis, University of British Columbia, Vancouver, Canada, 185p.

Uthayakumar, M., and Vaid, Y.P. (1998), "Static Liquefaction of Sands under Multiaxial Loading”, Canadian Geotechnical Journal, 35(2):273-283.

Vaid, Y.P., and Chern, J.C., (1983), "Mechanism of Deformation during Undrained Loading of Saturated Sand”, International Journal of Soil Dynamics and Earthquake Engineering, 2(3):171-177.

Vaid, Y.P., and Chern, J.C., (1985), “Cyclic and Monotonic Undrained Response of Sands", Proceedings Advances in the Art of Testing Soils under Cyclic Loading Conditions, Detroit, pp. 120-147.

Vaid, Y.P., and Eliadorani, A., (2000), "Undrained and Drained (?) Stress-Strain Response", Canadian Geotechnical Journal, 37(5):1126-1130.

Vaid, Y.P., and Negussey, D., (1988), "Preparation of Reconstituted Sand Specimens", Symposium on Advanced Triaxial Testing of Soil and Rock, ASTM STP 977, pp. 405417.

Vaid, Y.P., and Sivathayalan, S., (1996), "Static and Cyclic Liquefaction Potential of Fraser Delta Sand in Simple Shear and Triaxial Tests", Canadian Geotechnical Journal, 33(2):281-289.

Vaid, Y.P., and Sivathayalan, S., (2000), "Fundamental Factors Affecting Liquefaction Susceptibility of Sands". Canadian Geotechnical Journal, 37(3):592-606.

Vaid, Y.P., and Thomas, J., (1994), "Post Liquefaction Behaviour of Sand", Proceedings $13^{\text {th }}$ International Conference on Soil Mechanics and Foundation Engineering, New Delhi, India, pp. 1305-1310. 
Vaid, Y.P., and Thomas, J., (1995), "Liquefaction and Post Liquefaction of Sand", Journal of Geotechnical Engineering”, 121(2):163-173.

Vaid, Y.P., and Thomas, J., (1996), Closure on "Liquefaction and Post Liquefaction of Sand", Journal of Geotechnical Engineering, 122(2):503-504.

Vaid, Y.P., Chung, E.K.F., and Kuerbis, R.H., (1990a), "Stress Path and Steady State”, Canadian Geotechnical Journal, 27(1):1-7.

Vaid, Y.P., Sayao, A., Enhuang, H. and Negussey, D., (1990b), “Generalized Stress-PathDependent Soil Behaviour with a New Hollow Cylinder Torsional Apparatus”, Canadian Geotechnical Journal, 27(5):601-616.

Vaid, Y.P., Sivathayalan, S., and Stedman, D., (1999), "Influence of Specimen Reconstitution Method on Undrained Response of Sand", ASTM Geotechnical Testing Journal, 22(3):187-195.

Vaid, Y.P., Stedman, J.D., and Sivathayalan, S. (2001), "Confining Stress and Static Shear Effects in Cyclic Liquefaction”, Canadian Geotechnical Journal, 38(3):580-591.

Wijewickreme, J.D., and Vaid, P.J., (1991), "Stress Nonuniformity in Hollow Cylinder Torsional Specimen", Geotechnical Testing Journal, 14(4):349-362.

Wijewickreme, W.K.D. (1990). Ph.D. Thesis, "Behaviour of Sand under Simultaneous Increase in Stress Ratio and Principal Stress rotation", Department of Civil Engineering, University of British Columbia, Canada, 225p.

Wijewickreme, W.K.D., and Vaid, Y.P., (2008), "Experimental Observations on the Response of Loose Sand under Simultaneous Increase in Stress Ratio and Rotation of Principal Stresses", Canadian Geotechnical Journal, 45:597-610. 
Wong, R.K.S., and Arthur, J.R.F., (1985), "Induced and Inherent Anisotropy in Sand", Géotechnique, 35, (4):471-481.

Xia Li, Hai-Sui Yu, (2009), "Influence of Loading Direction on The Behaviour of Anisotropic Granular Materials”, International Journal of Engineering Science, (47):1284-1296.

Yamada, Y. and Ishihara, K., (1979), “Anisotropic Deformation Characteristics of Sand under Three Dimensional Stress Conditions", Soils and Foundations, 19(2):79-94.

Yang, Z.X., Li, X.S., and Yang, J., (2007) "Undrained Anisotropy and Rotational Shear in Granular Soil”, Géotechnique, 57(4):371-384.

Yoshimine, M., Ishihara, K., and Vargas, W., (1998), "Effects of Principal Stress Direction and Intermediate Principal Stress on Undrained Shear Behaviour of Sand", Soils and Foundations, 38(3):179-188. 
Appendix A

(Stress Paths) 
Behaviour for $\alpha_{\sigma c}=30^{\circ}$

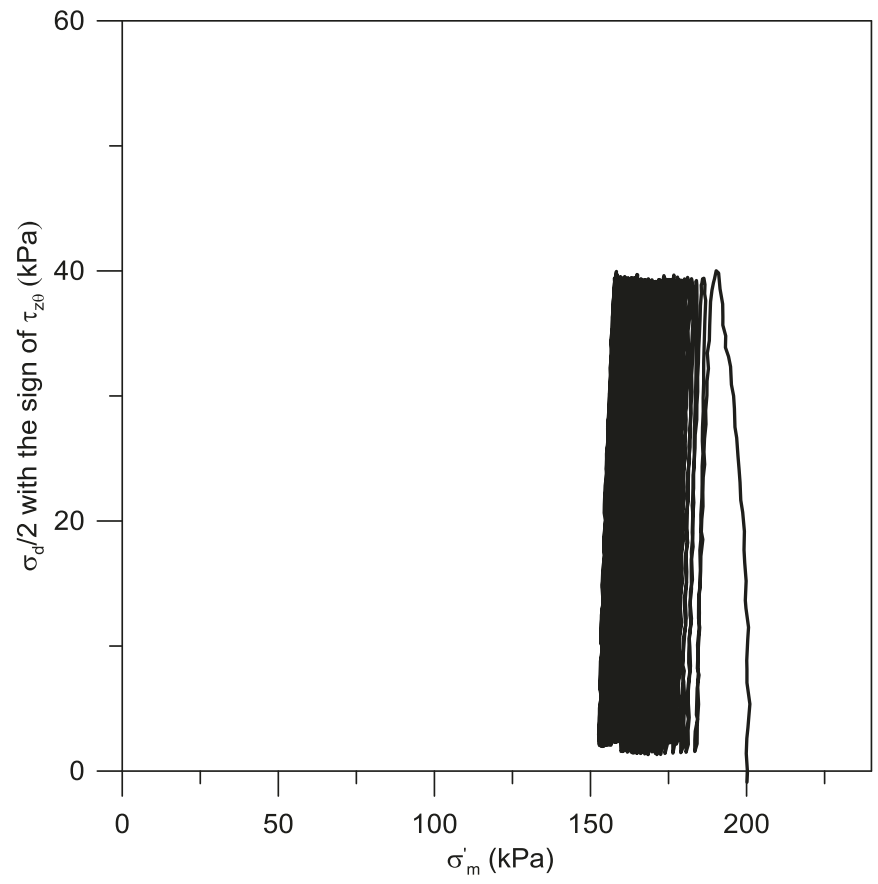

Fig. A.1 Stress path of Fraser River sand during rotation of $\alpha_{\sigma}=30_{-}^{+} 0^{\circ}$

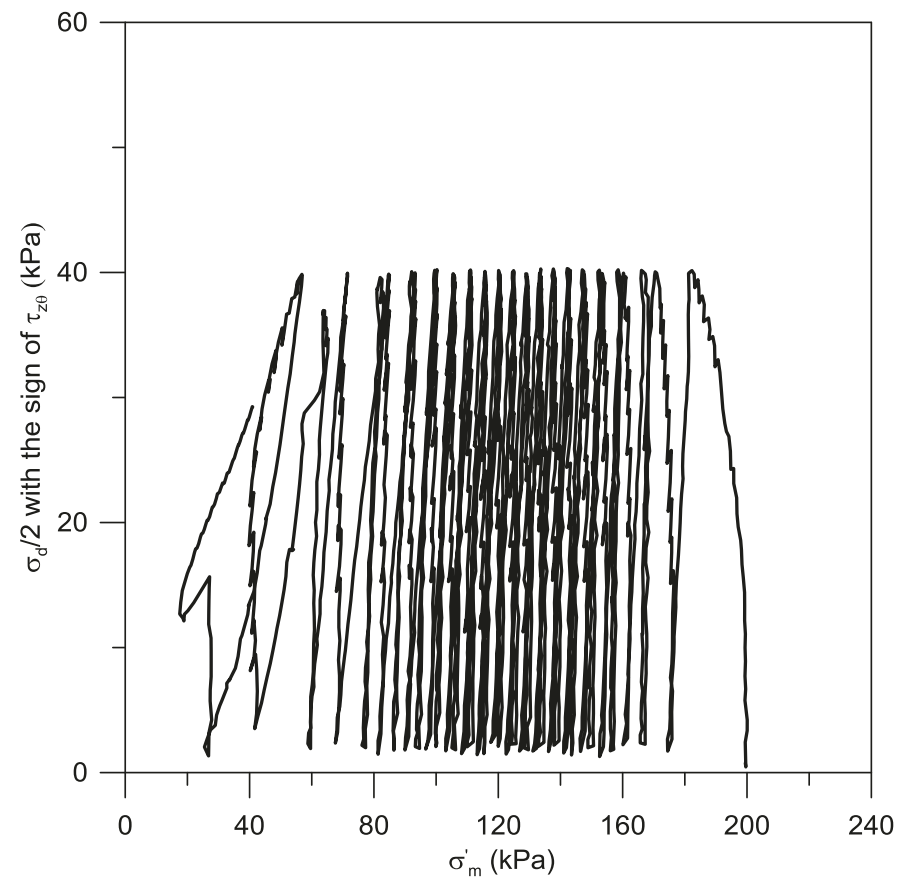

Fig. A.2 Stress path of Fraser River sand during rotation of $\alpha_{\sigma}=30_{-}^{+} 30^{\circ}$ 


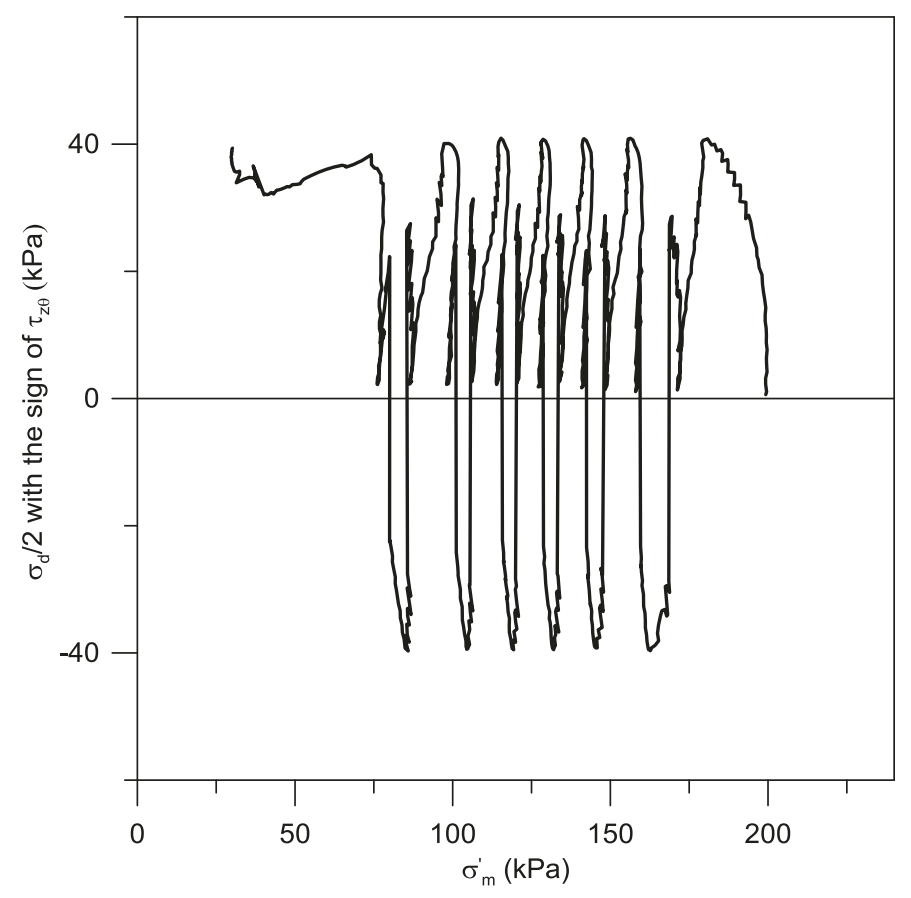

Fig. A.3 Stress path of Fraser River sand during rotation of $\alpha_{\sigma}=30_{-}^{+} 45^{\circ}$

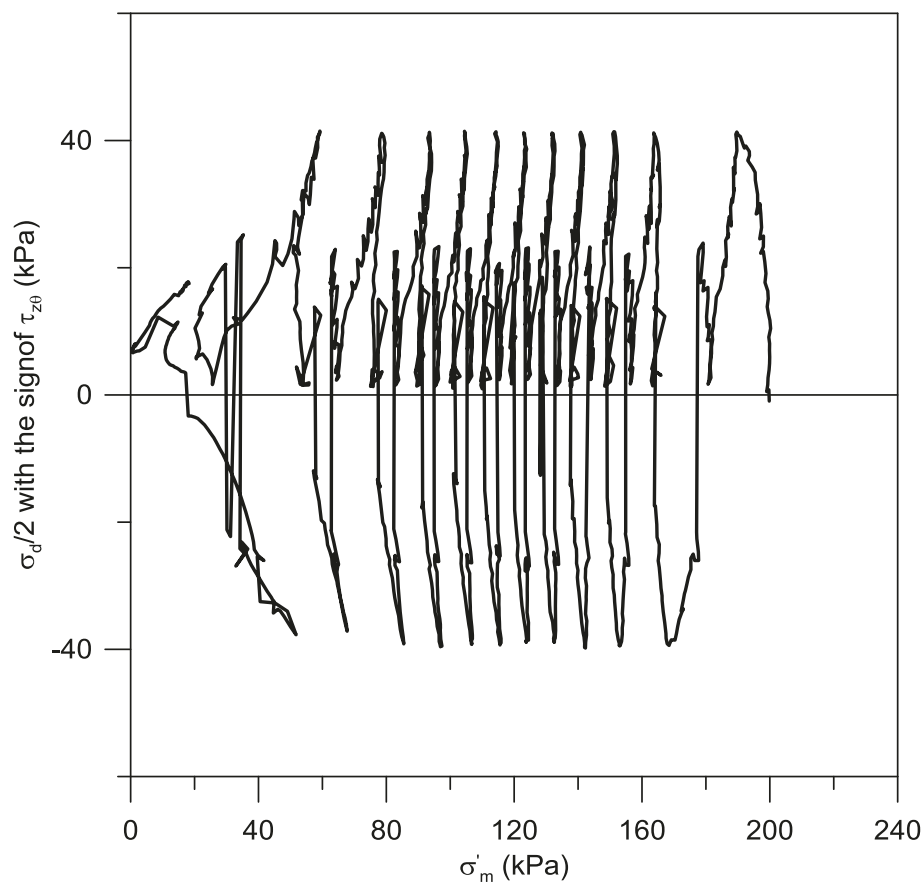

Fig. A.4 Stress path of Fraser River sand during rotation of $\alpha_{\sigma}=30_{-}^{+} 60^{\circ}$ 


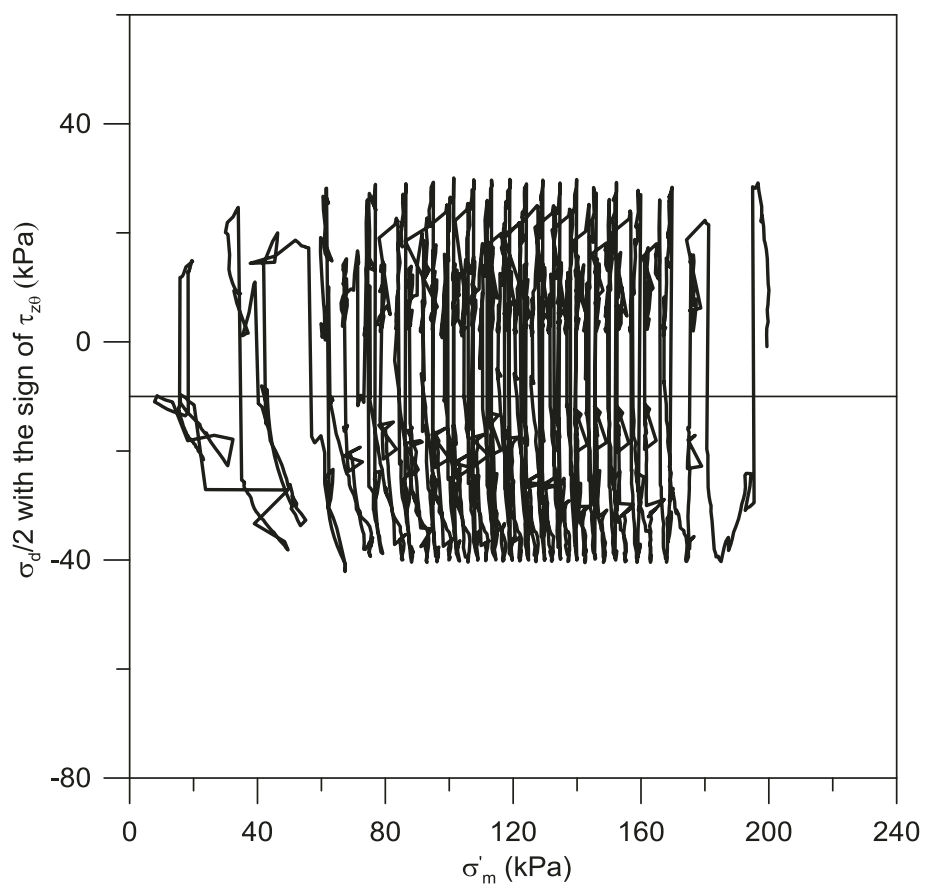

Fig. A.5 Stress path of Fraser River sand during rotation of $\alpha_{\sigma}=30_{-}^{+90^{\circ}}$ Behaviour for $\alpha_{\sigma c}=45^{\circ}$

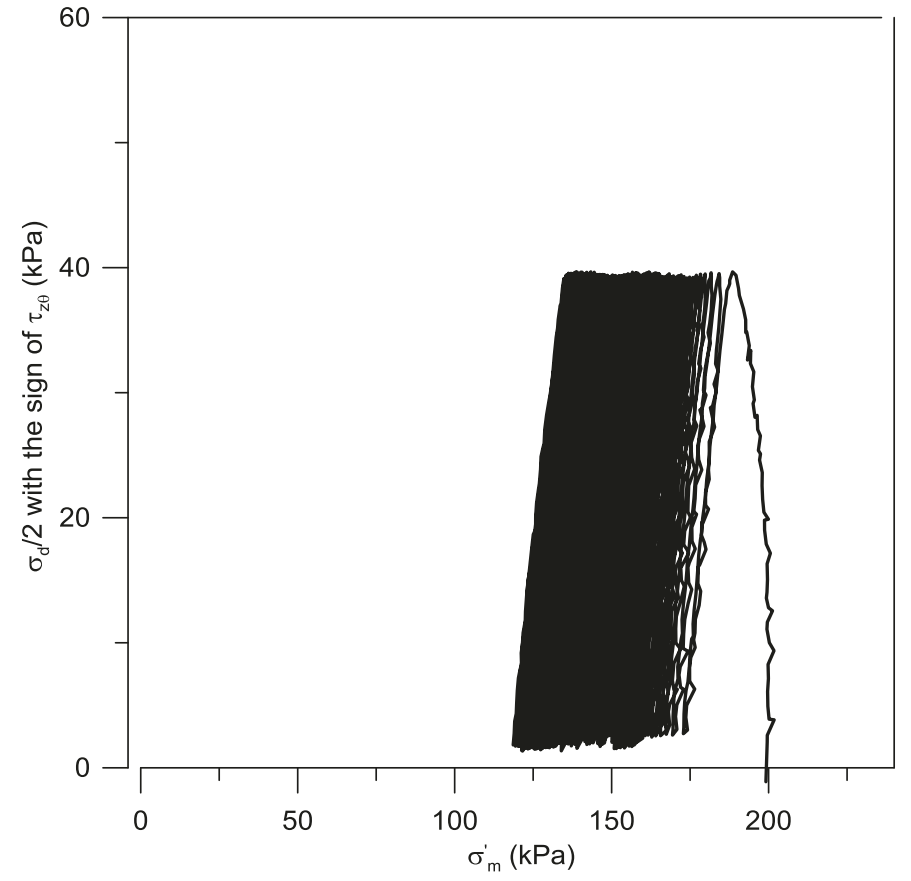

Fig. A.6 Stress path of Fraser River sand during rotation of $\alpha_{\sigma}=45_{-}^{+} 0^{\circ}$ 


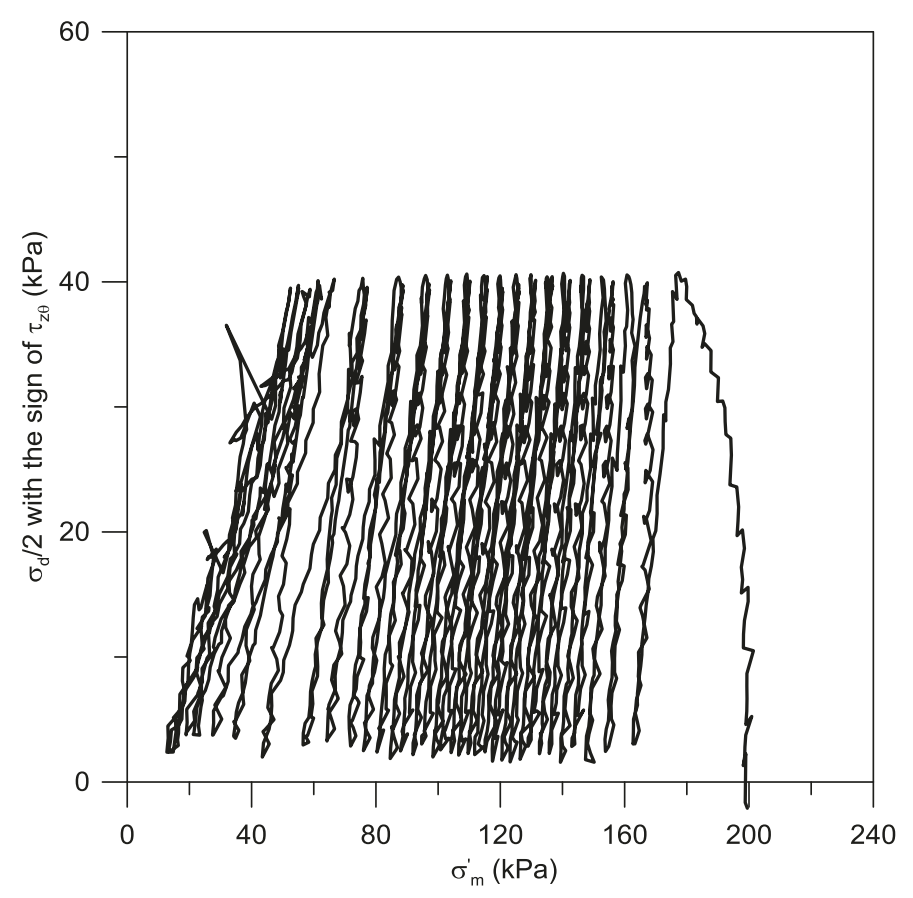

Fig. A.7 Stress path of Fraser River sand during rotation of $\alpha_{\sigma}=45_{-}^{+} 30^{\circ}$

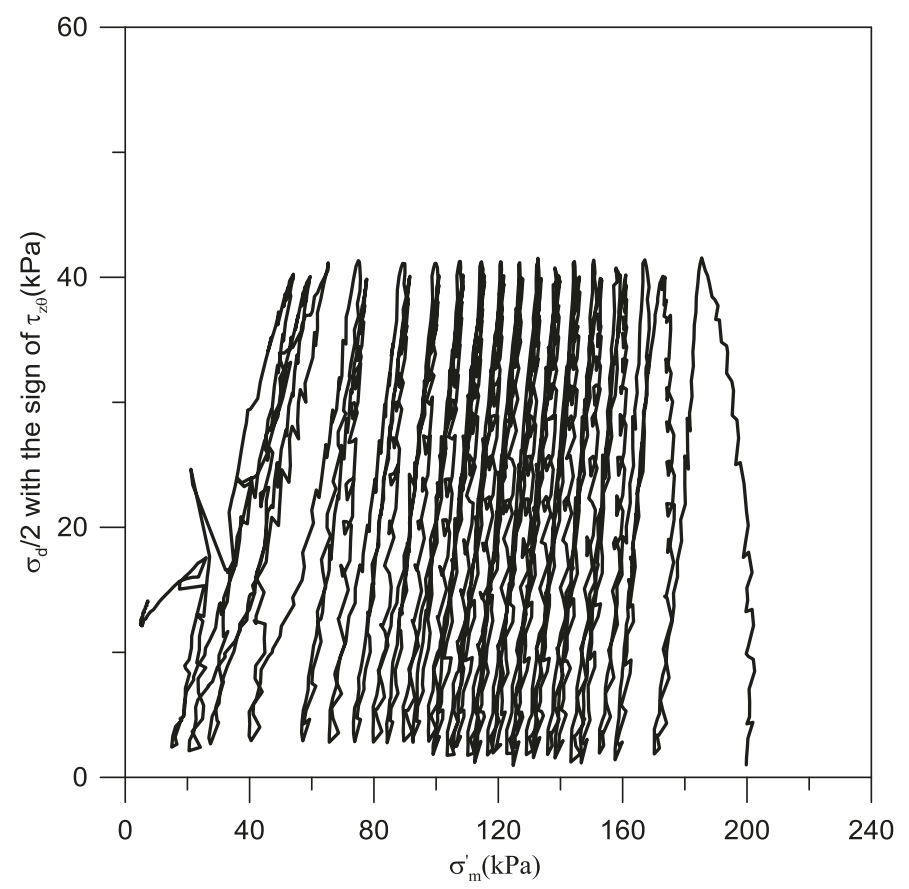

Fig. A.8 Stress path of Fraser River sand during rotation of $\alpha_{\sigma}=45_{-}^{+} 45^{\circ}$ 


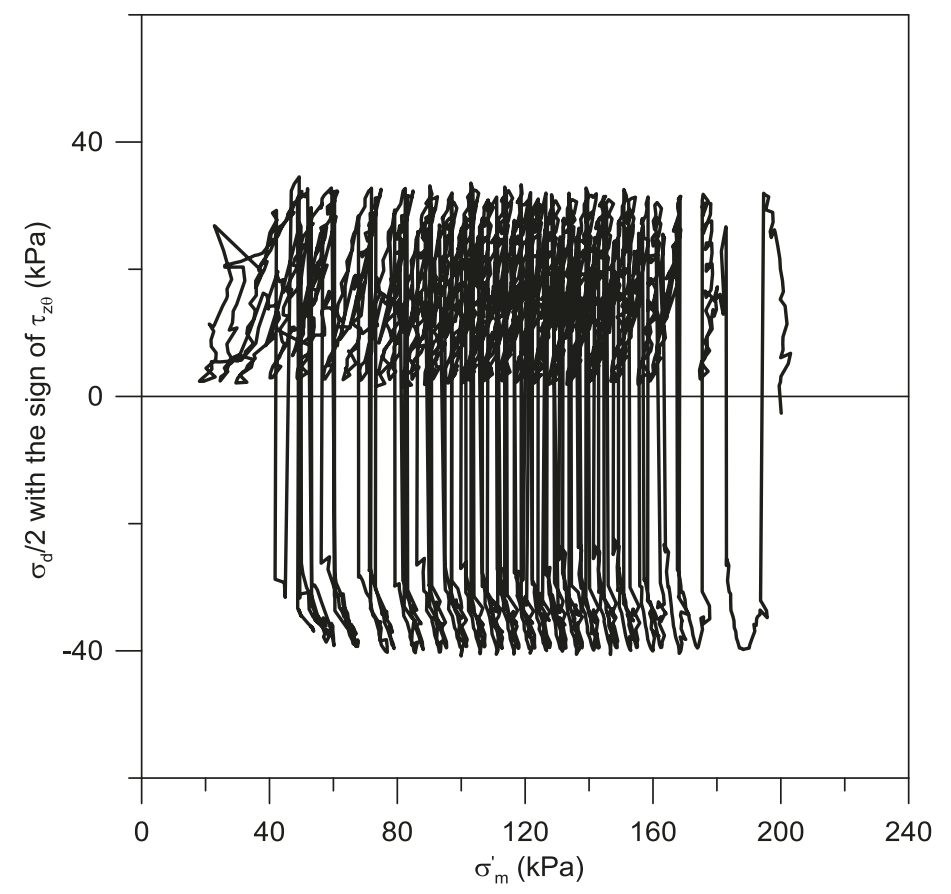

Fig. A.9 Stress path of Fraser River sand during rotation of $\alpha_{\sigma}=45_{-}^{+} 60^{\circ}$

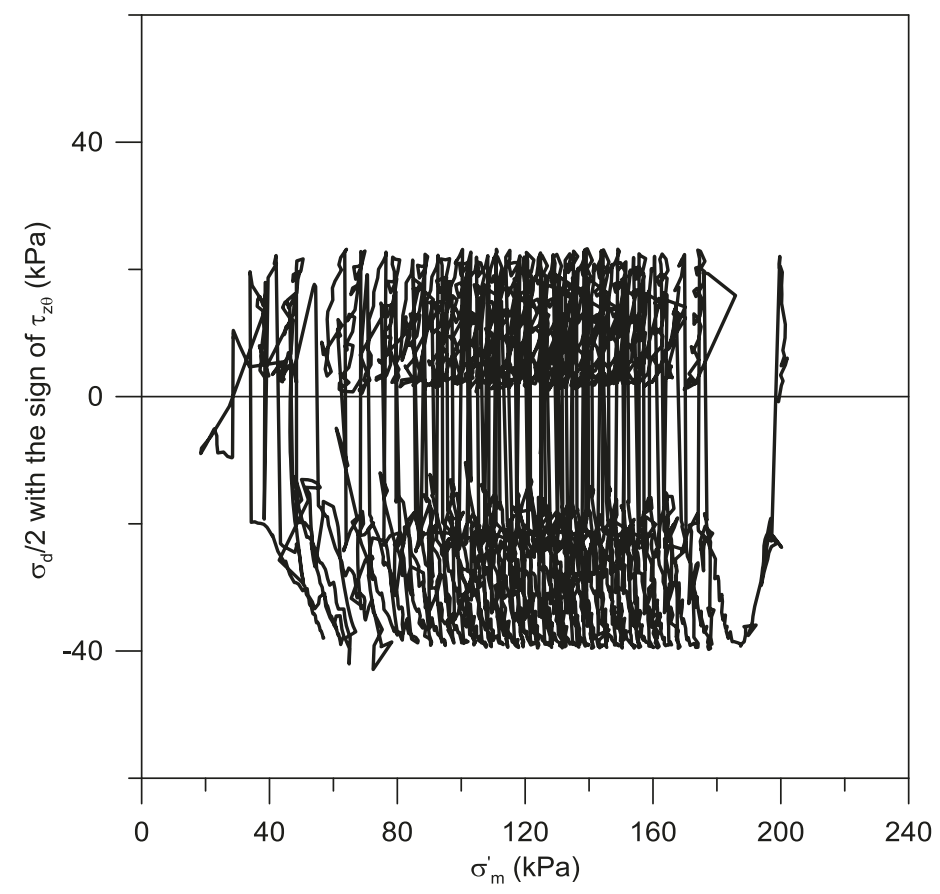

Fig. A.10 Stress path of Fraser River sand during rotation of $\alpha_{\sigma}=45_{-}^{+} 90^{\circ}$ 
Behaviour for $\alpha_{\sigma c}=60^{\circ}$

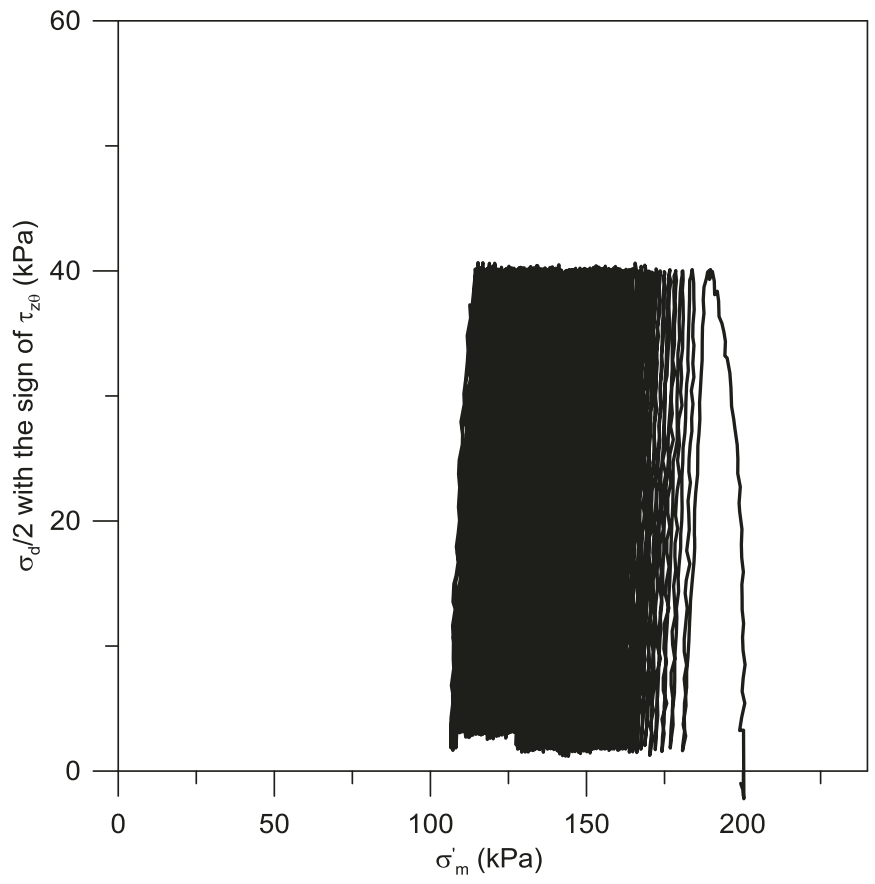

Fig. A.11 Stress path of Fraser River sand during rotation of $\alpha_{\sigma}=60_{-}^{+} 0^{\circ}$

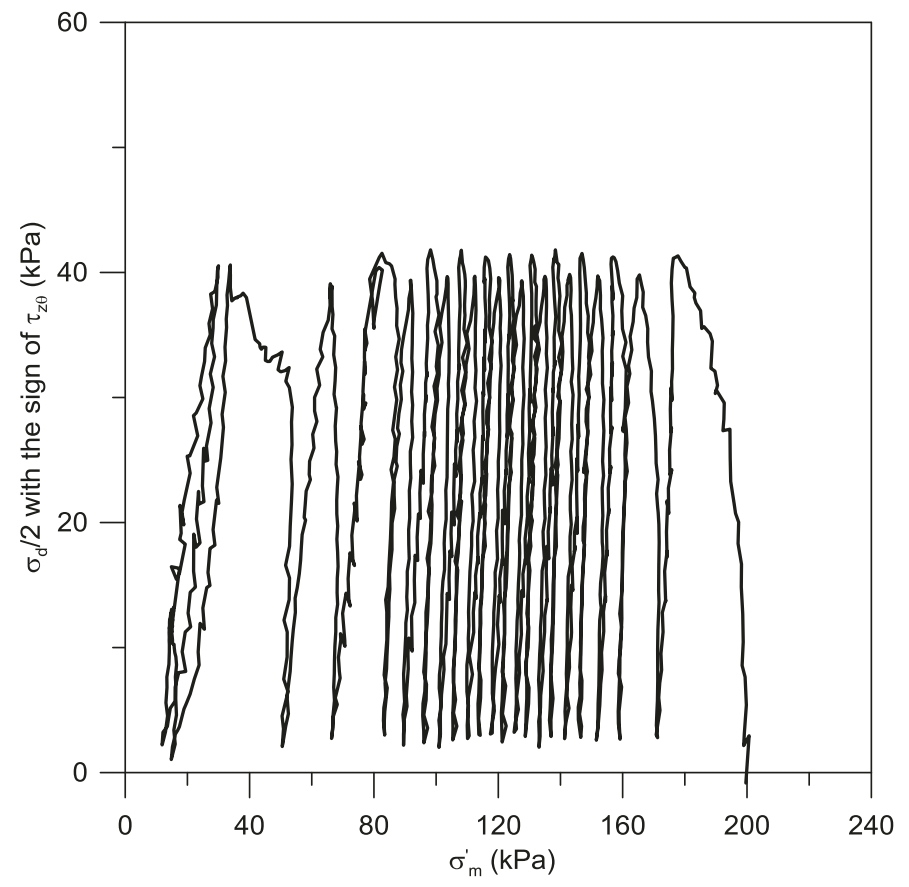

Fig. A.12 Stress path of Fraser River sand during rotation of $\alpha_{\sigma}=60_{-}^{+30^{\circ}}$ 


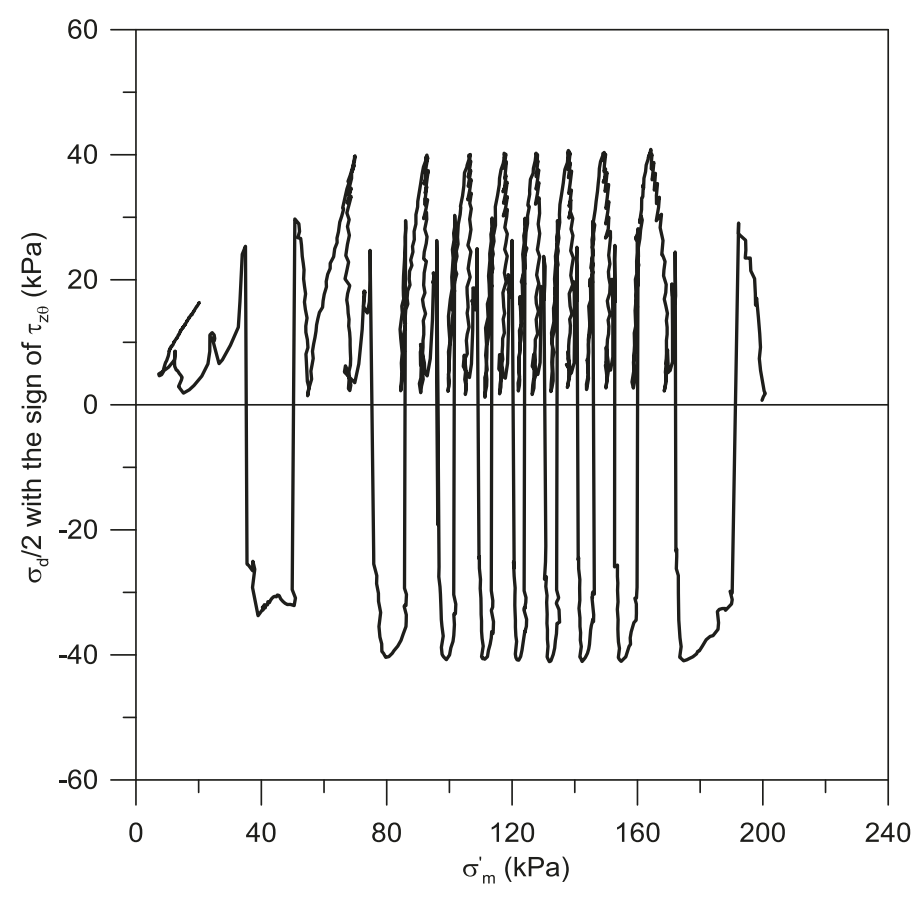

Fig. A.13 Stress path of Fraser River sand during rotation of $\alpha_{\sigma}=60_{-}^{+} 45^{\circ}$

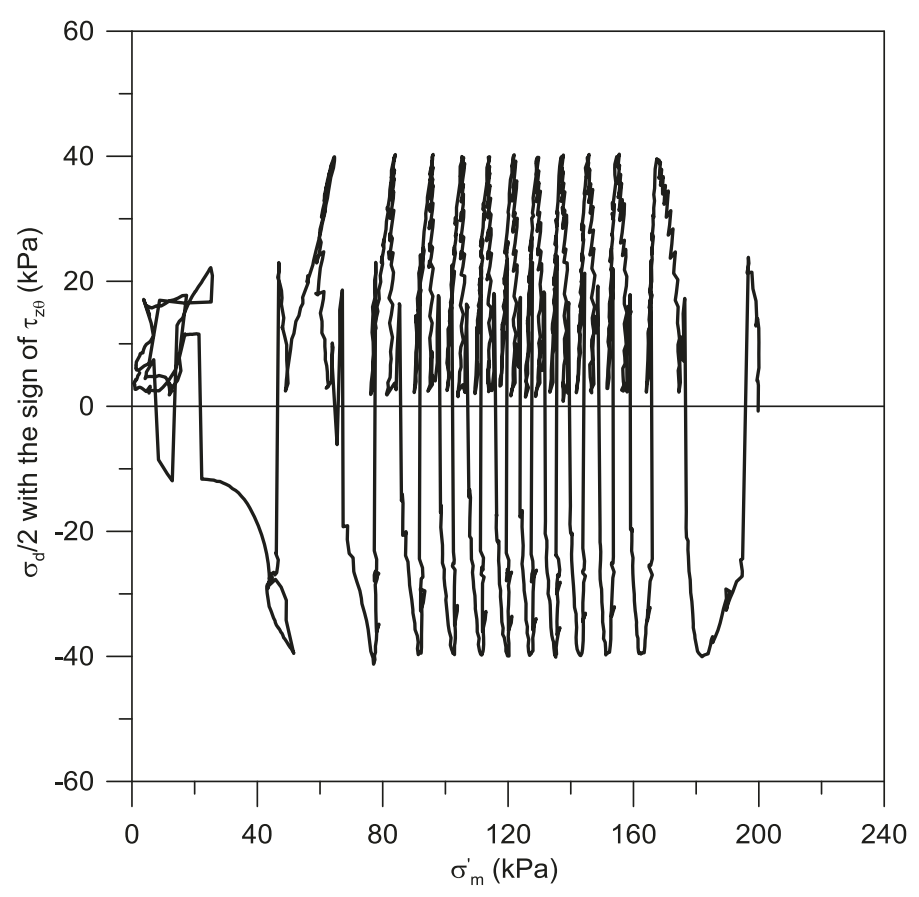

Fig. A.14 Stress path of Fraser River sand during rotation of $\alpha_{\sigma}=60_{-}^{ \pm} 60^{\circ}$ 


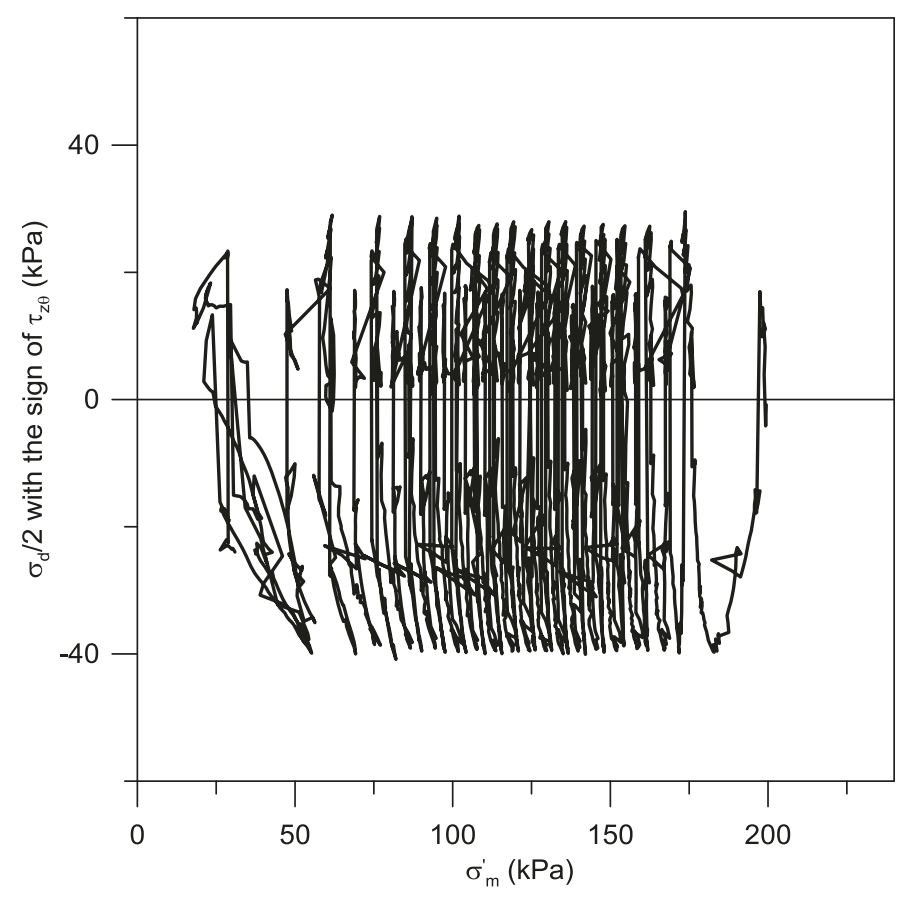

Fig. A.15 Stress path of Fraser River sand during rotation of $\alpha_{\sigma}=60_{-}^{+9} 0^{\circ}$ Behaviour for $\alpha_{\sigma c}=90^{\circ}$

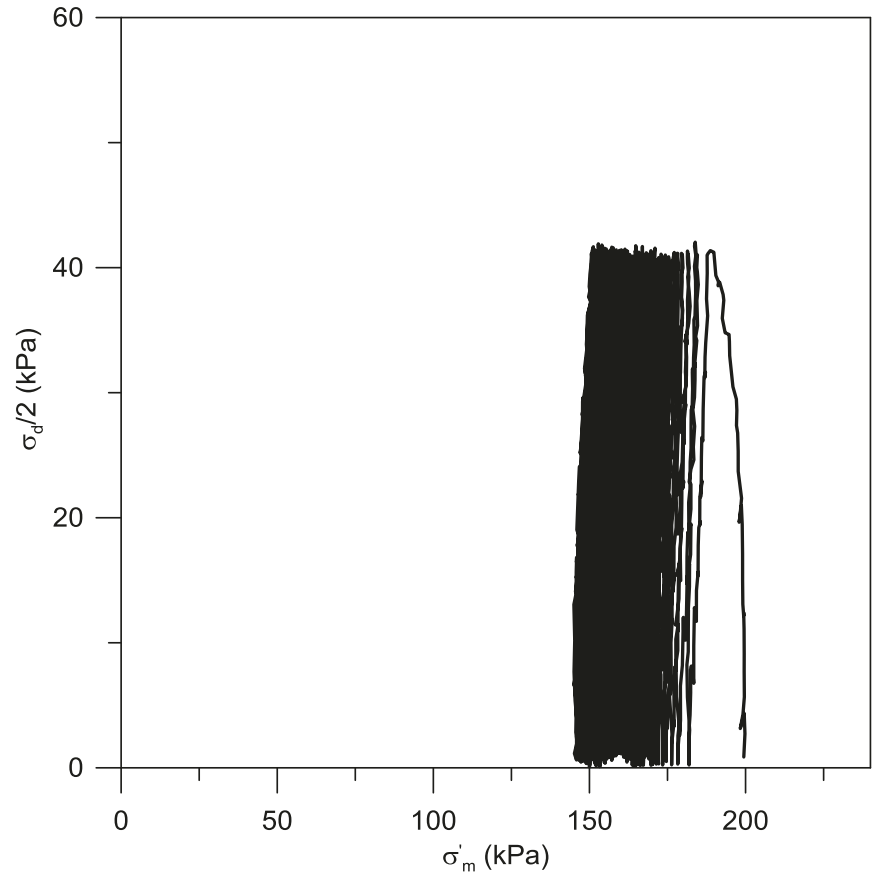

Fig. A.16 Stress path of Fraser River sand during rotation of $\alpha_{\sigma}=90_{-}^{+} 0^{\circ}$ 


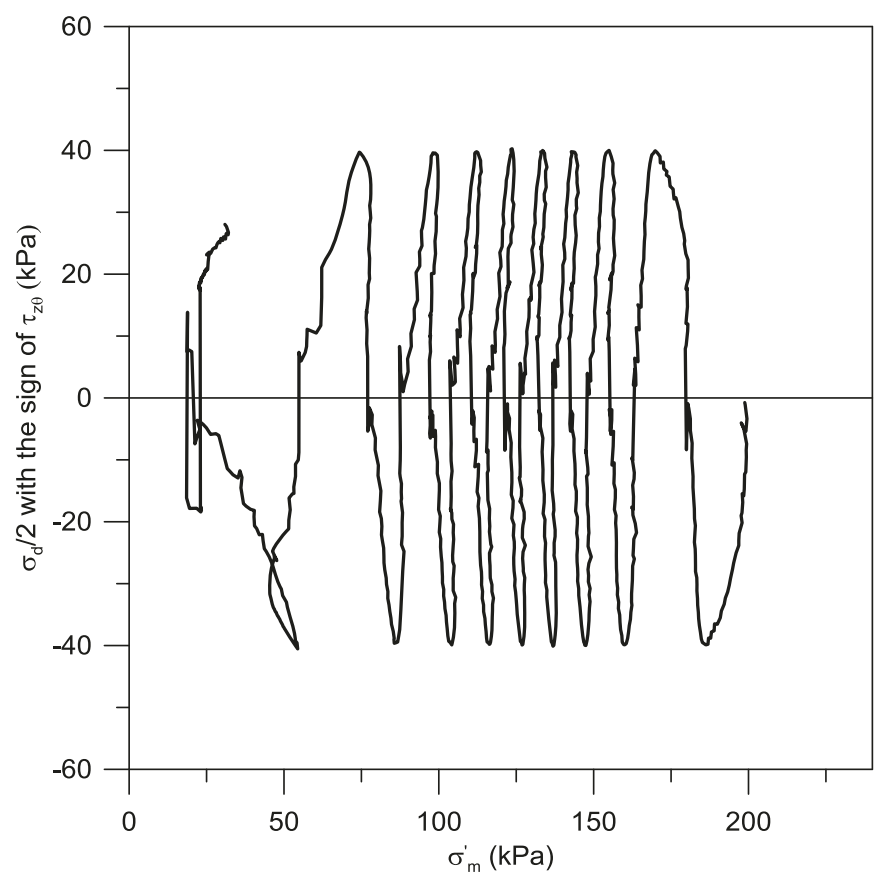

Fig. A.17 Stress path of Fraser River sand during rotation of $\alpha_{\sigma}=90_{-}^{+} 30^{\circ}$

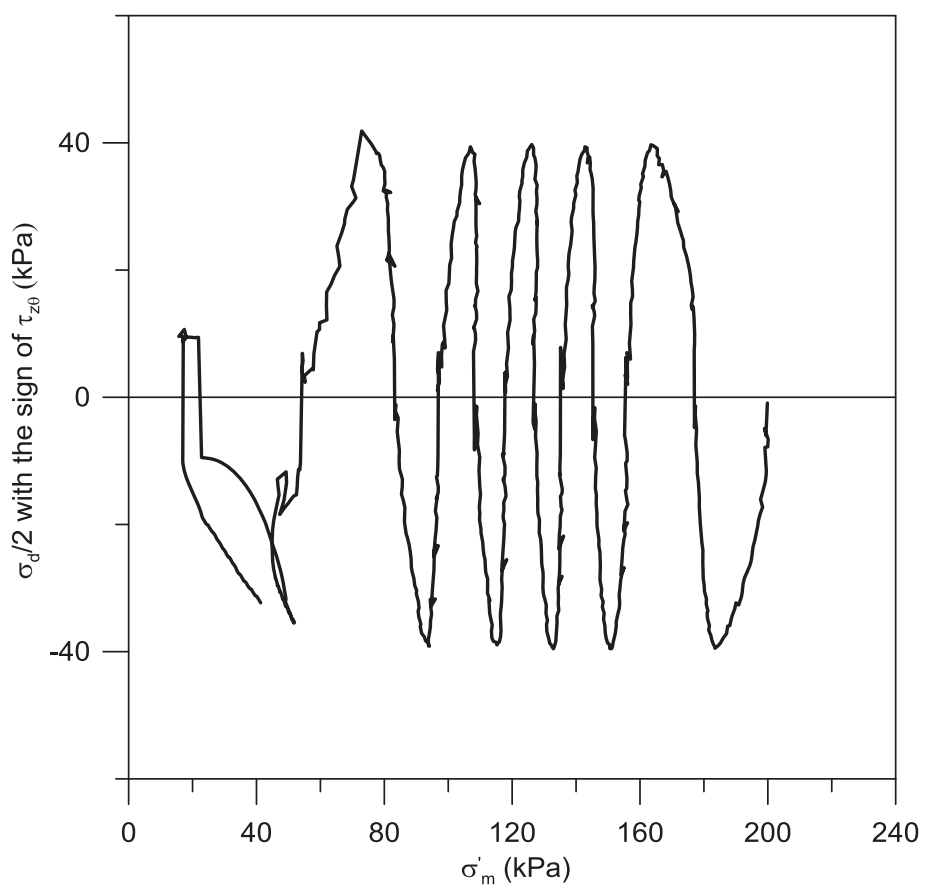

Fig. A.18 Stress path of Fraser River sand during rotation of $\alpha_{\sigma}=90_{-}^{+} 45^{\circ}$ 


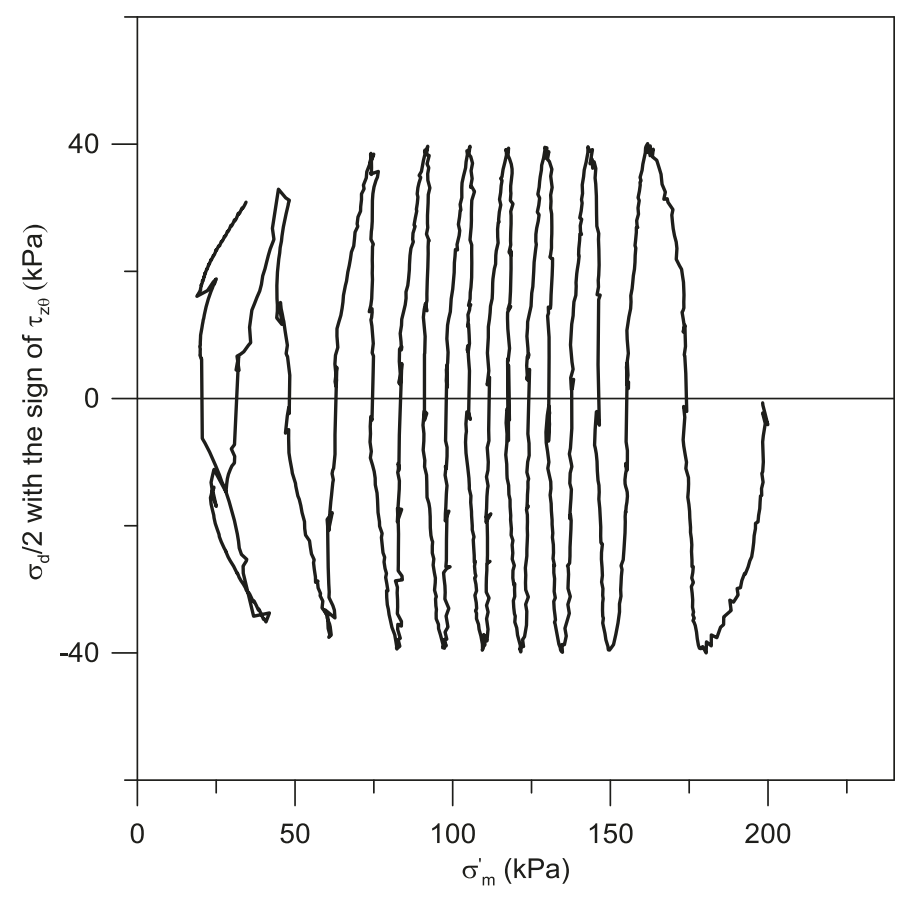

Fig. A.19 Stress path of Fraser River sand during rotation of $\alpha_{\sigma}=90_{-}^{ \pm} 60^{\circ}$

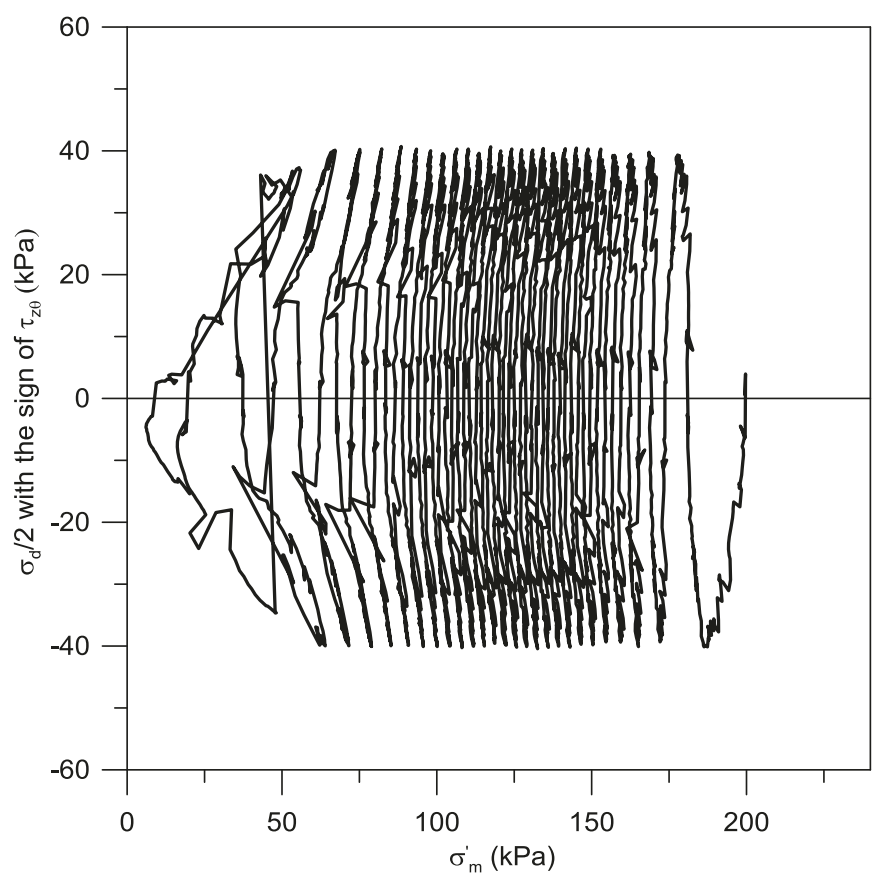

Fig. A.20 Stress path of Fraser River sand during rotation of $\alpha_{\sigma}=90_{-}^{+} 90^{\circ}$ 


\section{Appendix B}

(Excess PWP \& Maximum shear strain) 

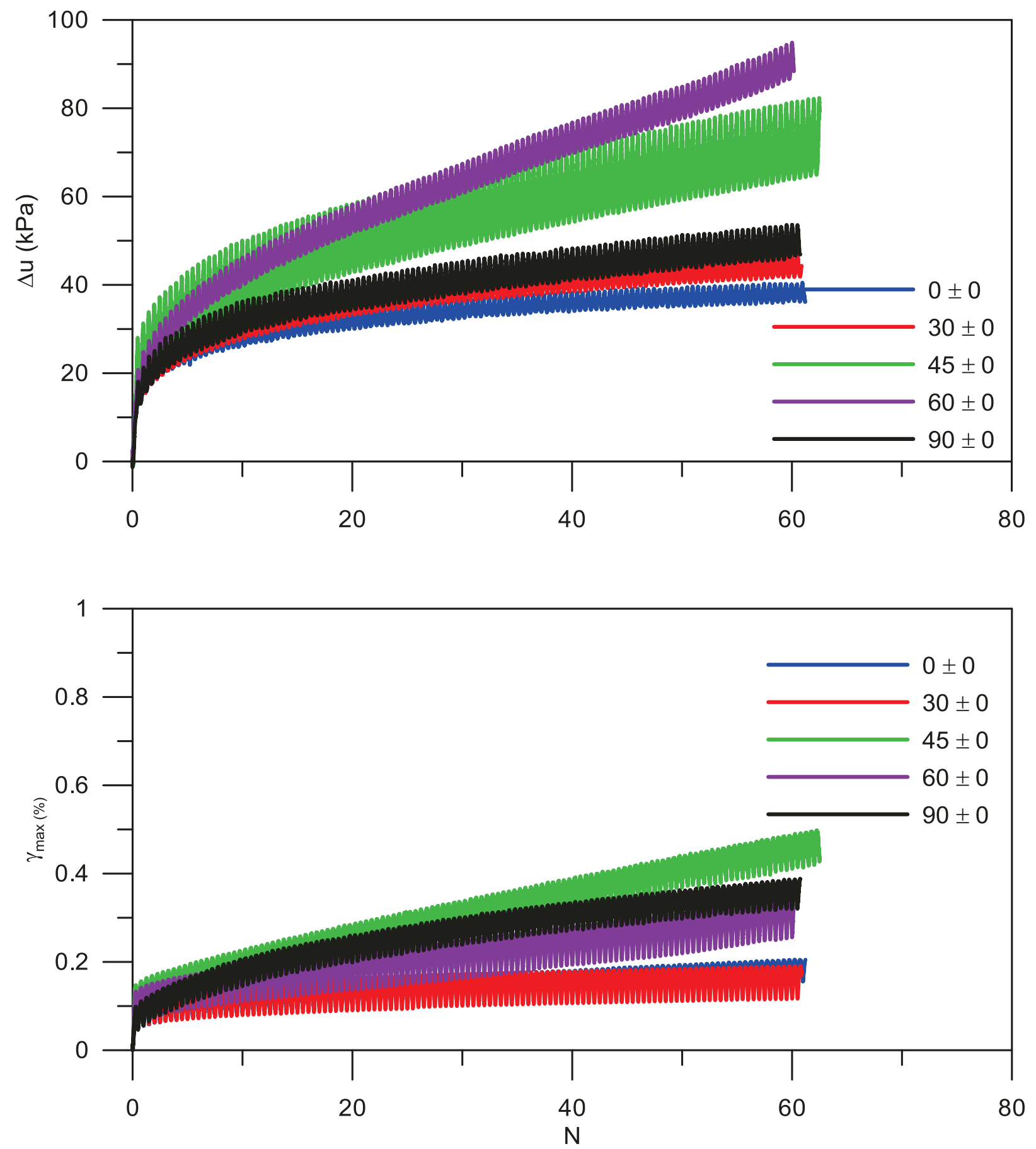

Fig. B.1 Excess pore pressure and maximum shear strain response of Fraser River sand during cyclic loading with $\Delta \alpha_{\sigma}=0^{\circ}$ 

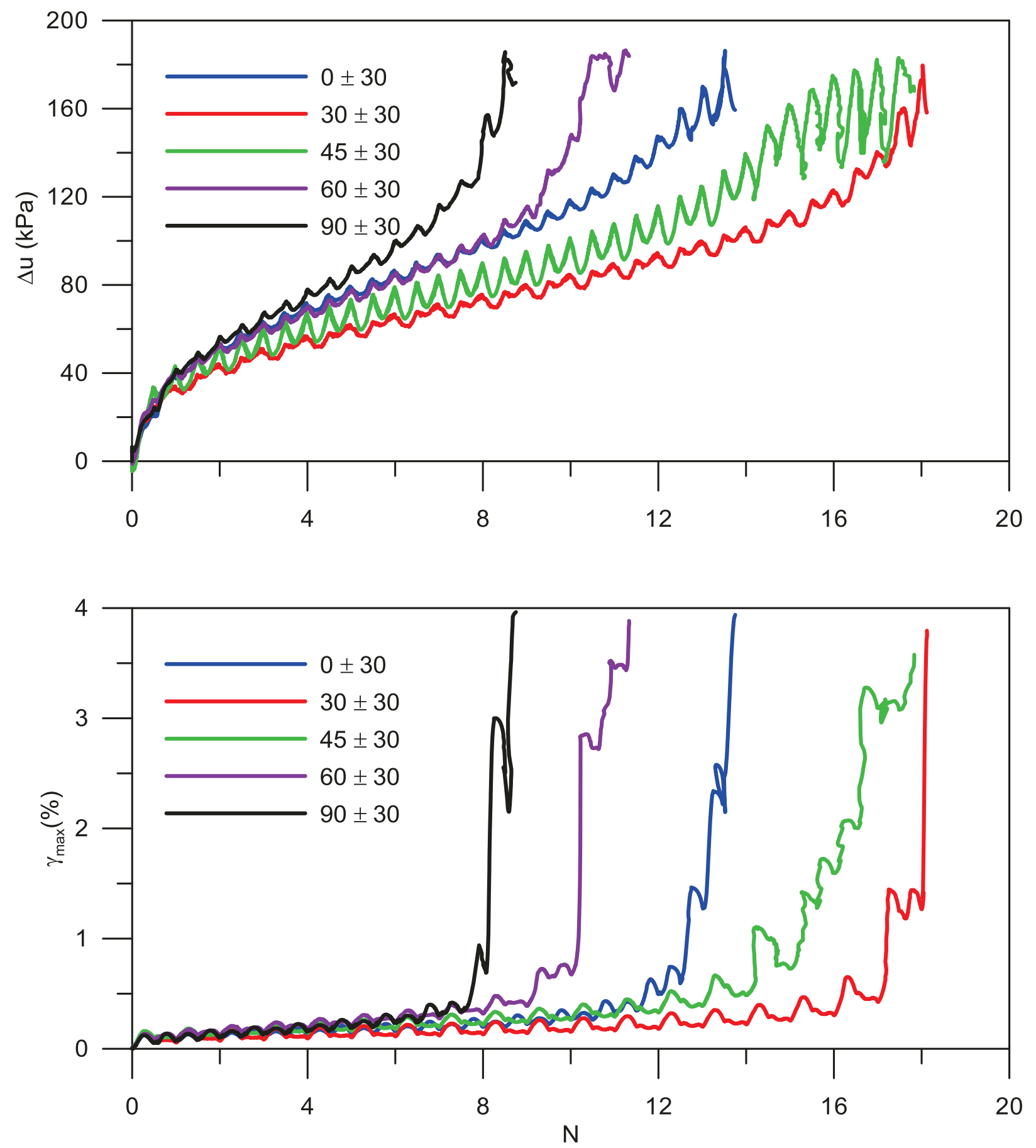

Fig. B.2 Excess pore pressure and maximum shear strain response of Fraser River sand during cyclic loading with $\Delta \alpha_{\sigma}=30^{\circ}$ 

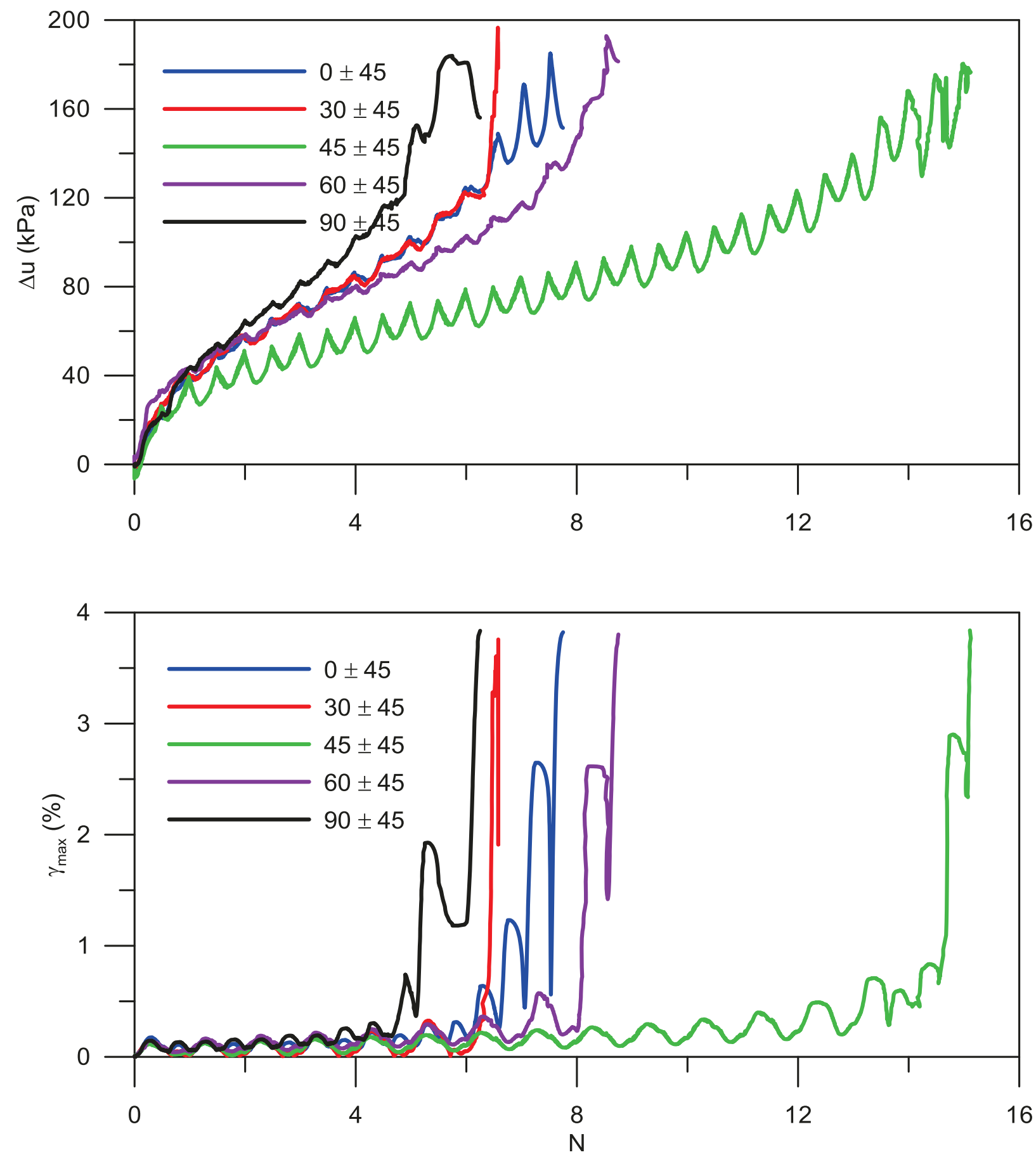

Fig. B.3 Excess pore pressure and maximum shear strain response of Fraser River sand during cyclic loading with $\Delta \alpha_{\sigma}=45^{\circ}$ 

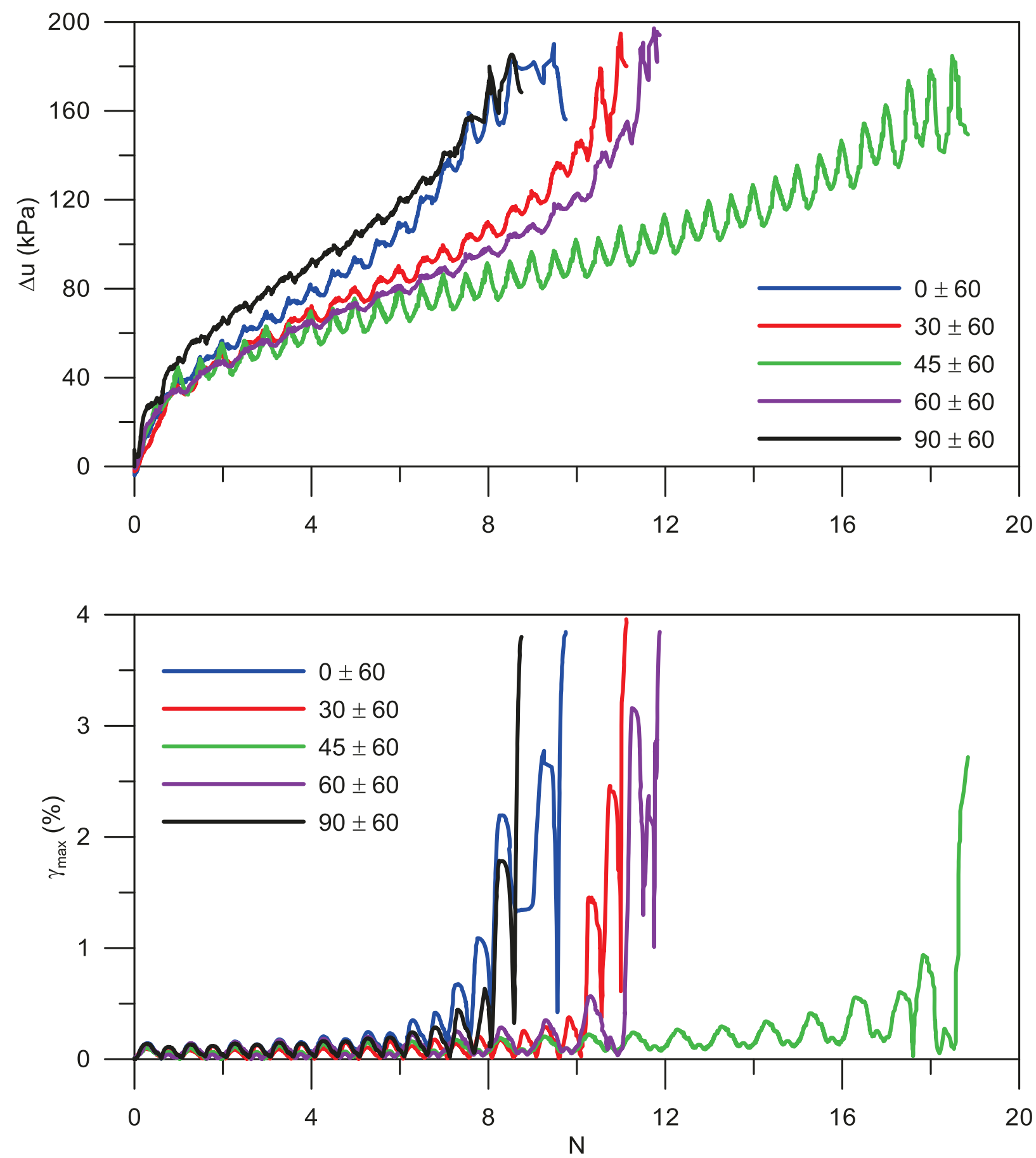

Fig. B.4 Excess pore pressure and maximum shear strain response of Fraser River sand during cyclic loading with $\Delta \alpha_{\sigma}=60^{\circ}$ 

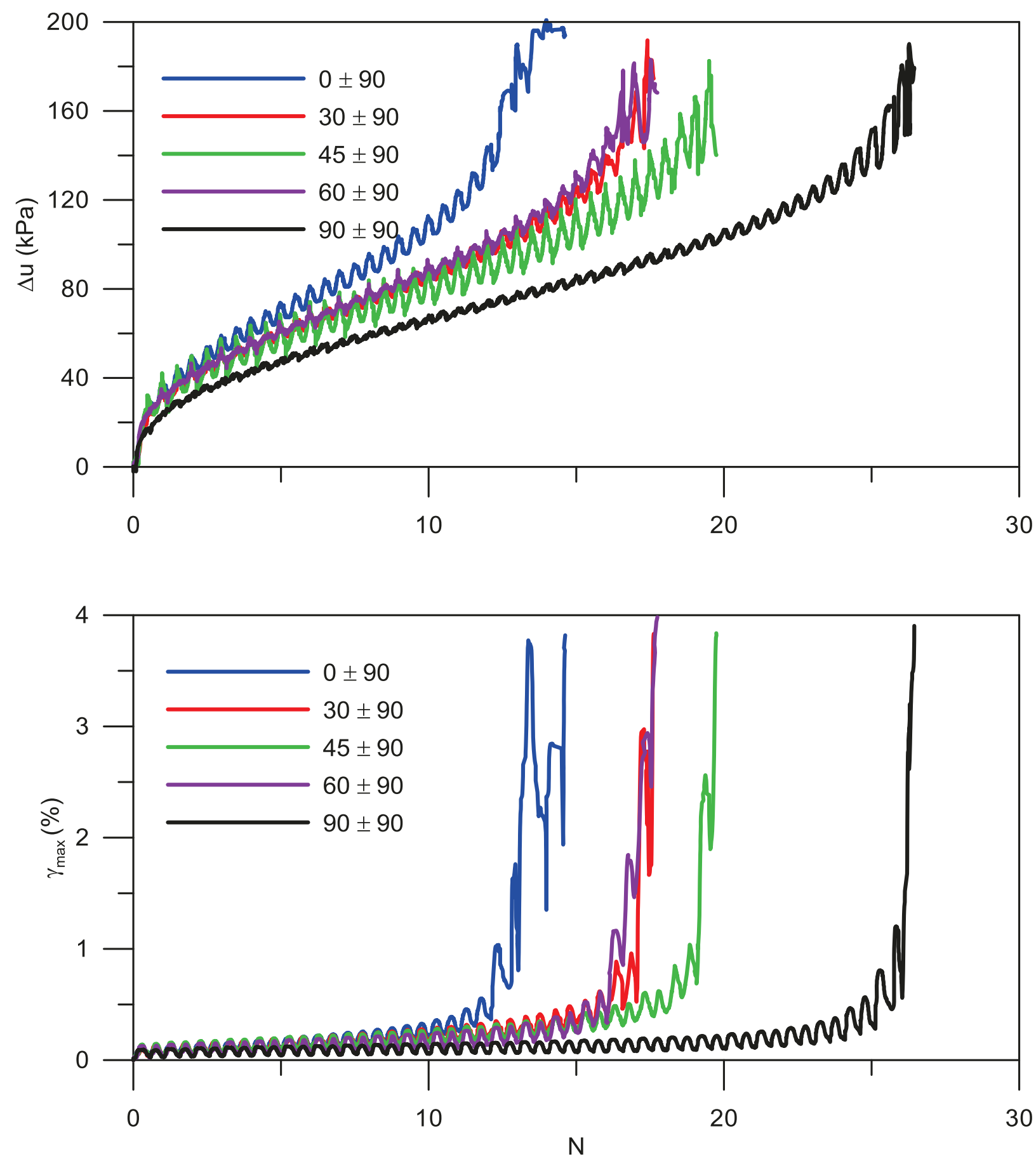

Fig. B.5 Excess pore pressure and maximum shear strain response of Fraser River sand during cyclic loading with $\Delta \alpha_{\sigma}=90^{\circ}$ 